\title{
The Cellular and Physiological Basis of Host - Symbiont Specificity in a Model Cnidarian-Dinoflagellate Symbiosis
}

\author{
Yasmin Gabay
}

A thesis submitted to Victoria University of Wellington in fulfilment of the requirements for the degree of Doctor of Philosophy in Science

Victoria University of Wellington

2018

VICTORIA UNIVERSITY OF WELLINGTON

Te Whare Wānanga o te Ūpoko o te Ika a Mãui

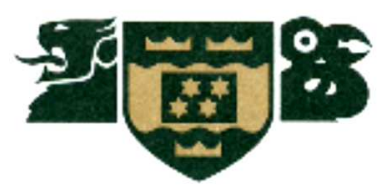





\section{Abstract}

The ability of corals to form novel partnerships with symbionts that may be better suited to new environmental conditions is an important factor when assessing the ability for corals to adapt to climate change. However, relatively little attention has been given to the effects of hosting different symbiont types on holobiont physiology, competitive interactions between these symbionts, or the capacity of the host to regulate populations of different symbionts. Such factors likely play an important role in patterns of host-symbiont specificity and flexibility, and hence the potential for corals to respond to climate change. The aim of this research was to characterise the cellular and physiological events associated with hosting different symbiont species in Exaiptasia pallida (commonly referred to as 'Aiptasia'), a model cnidarian-dinoflagellate symbiosis, and how these events might contribute to host-symbiont specificity. The specific objectives were to: (1) determine the effect of symbiont species on the population dynamics of host colonisation and holobiont physiology; (2) measure the competitiveness of the homologous symbiont versus heterologous symbionts, under both control and elevated temperatures; and quantify the ability of the host to regulate its symbiont population in response to homologous versus heterologous symbiont taxa (3) via host-cell apoptosis and (4) via symbiont cell cycle regulation.

To explore this, aposymbiotic (i.e. symbiont-free) individuals of Aiptasia were first inoculated with one of five Symbiodinium taxa (the homologous $S$. minutum or heterologous $S$. microadriaticum, phylotype $\mathrm{C} 3, S$. trenchii or $S$. voratum), and the rates and patterns of colonisation assessed. Proliferation success inside the anemone was different between symbionts, with the homologous $S$. minutum being the most successful species, while Symbiodinium C3 and S. voratum struggled or failed to form a long-lasting symbiosis. The spatial pattern of symbiont colonisation was identical for all the successful Symbiodinium taxa, however the timing differed between these different symbionts. Symbiont identity also had an effect on holobiont fitness, as $S$. microadriaticum and $S$. trenchii were less beneficial to the host compared to $S$. minutum, as indicated by lower rates of photosynthesis, anemone growth and pedal laceration (i.e. asexual reproduction). The taxon-specific differences demonstrated here provided a basis for the subsequent thesis chapters, leading to questions about how the different symbionts might compete with one another and be regulated by the host.

The competitiveness of the homologous symbiont relative to heterologous ones, and hence the ability of the host to 'switch' and 'shuffle' its symbiont population, was tested by inoculating aposymbiotic sea anemones either with simultaneous or sequential mixtures of thermally tolerant 
and sensitive Symbiodinium and exposing them to control versus elevated temperatures. The homologous species was dominant regardless of temperature, outcompeting the heterologous, thermally tolerant $S$. microadriaticum and S. trenchii. This result indicates that the high level of specificity seen between Aiptasia and S. minutum in the Pacific Ocean may result, in part, from a reluctance to form new symbioses, even if such associations have the potential to confer a degree of thermal tolerance that may be beneficial under future climate change.

The differential success of the different symbionts was also reflected in the host's apoptotic response to their presence in its tissues, as measured via caspase-3 activity. In particular, anemones hosting S. minutum and S. microadriaticum exhibited lower levels of caspase activity than those hosting S. trenchii and S. voratum throughout symbiosis establishment, consistent with symbiont proliferation success. The general pattern of caspase activity during the 28-days colonisation period was similar, however, with induction of caspase-3 activity upon inoculation, followed by a marked decline in activity over the subsequent week, and then an increase (either moderate or marked depending upon symbiont identity) across the remainder of the colonisation period measured. Host cell apoptosis therefore likely plays an important role in determining the compatibility and fate of different Symbiodinium taxa in a host, and the potential for establishing novel symbioses.

In contrast to the apparent importance of host apoptosis, symbiont cell cycle control did not seem to play an important role in determining the different rates of symbiont colonisation observed. Flow cytometry was used to determine the relative proportion of cells in the different phases of the cell cycle (i.e. $\mathrm{G}_{1}, \mathrm{G}_{2}, \mathrm{~S}, \mathrm{M}$ ), with all symbiont taxa exhibiting the same pattern of cell cycle progression. In particular, more cells were in the $S$ and $G_{2} / M$ phases combined than in $G_{1}$ during the first two weeks of colonisation, but this changed as colonisation progressed, when a greater proportion cells were in the $\mathrm{G}_{1}$ phase. This indicates that symbiont cell division becomes limited in the later stages of colonisation as symbiont density increases, consistent with increasing resource limitation.

This thesis provides valuable insights into the regulation of the cnidarian-dinoflagellate symbiosis, and the events that contribute to host-symbiont specificity. In particular, it suggests that through cellular control and physiological impacts on the host (and hence the overall symbiosis), there is likely limited potential to establish new host-symbiont partnerships that allow for adaptation to our warming climate. The next step is now to further elucidate the relative importance of post-phagocytosis control mechanisms, and to test the generality of my findings by extending them from the model Aiptasia system to reef corals. 


\section{Contributions and Publications}

This thesis is written as a series of manuscripts which are either already published, submitted, or in preparation for submission in the near future. As a result, there is some repetition of themes between chapters (particularly in the "Introduction" and "Material and Methods" sections). All laboratory work, statistical analyses and writing were conducted by the Author, with assistance as described below.

\section{Chapter 2}

This chapter is formatted as a stand-alone manuscript and is accepted for publication in the journal The Biological Bulletin: Gabay, Y., Weis, V. M. and Davy, S. K. (In Press. See Appendix 1). "Symbiont type influences patterns of symbiosis establishment, host growth and asexual reproduction in a model cnidarian-dinoflagellate symbiosis." All laboratory work and analyses were completed by the Author. V. M. Weis and S. K. Davy advised on experimental set-up, analyses and writing.

\section{Chapter 3}

This chapter is formatted as a stand-alone manuscript and will be submitted for publication in the journal Global Change Biology: Gabay, Y., Parkinson J. E., Wilkinson, S. P., Weis, V. M., and Davy, S. K. (in prep.). "Symbiont competition under thermal stress: host-symbiont specificity is the key factor that determined the faith of the symbiosis." All laboratory work and analyses were completed by the Author. J. E. Parkinson assisted with the statistical analysis and manuscript revision, S. P. Wilkinson assisted with the quantitative PCR, and V. M. Weis and S. K. Davy advised on experimental set-up, analyses and writing. 


\section{Chapter 4}

This chapter is formatted as a stand-alone manuscript and will be submitted for publication: Gabay, Y., Weis, V. M., and Davy, S. K. (in prep.). "The role of host cell apoptosis as a postphagocytosis mechanism of symbiont control in the cnidarian-dinoflagellate symbiosis." All laboratory work and analyses were completed by the Author. V. M. Weis and S. K. Davy advised on experimental set-up, analyses and writing.

\section{Chapter 5}

This chapter is formatted as a stand-alone manuscript and will be submitted for publication: Gabay, Y., Tivey, T., Weis, V. M., and Davy, S. K. (in prep.). "The role of symbiont cell cycle control in a model cnidarian-dinoflagellate." All laboratory work and analyses were completed by the Author. T. Tivey assisted with flow cytometry and advised on data analysis. V. M. Weis and S. K. Davy advised on experimental set-up, analyses and writing. 


\section{Acknowledgements}

In the completion of my $\mathrm{PhD}$ I would like to thank so many colleagues, friends and family members who provided support throughout my thesis, not all of whom I am able to give particular mention to here. Without their constant encouragement, love and support it would have been much harder to complete this $\mathrm{PhD}$.

Firstly, I would like to thank Victoria University of Wellington for awarding me the VUW $\mathrm{PhD}$ scholarship and enabling me to come to New Zealand to pursue this $\mathrm{PhD}$ project.

I would like to express my gratitude to my supervisor, Prof. Simon Davy, for his enthusiasm and support that enabled me to construct this $\mathrm{PhD}$ project. His assistance, encouragement and feedback throughout the course of my study, especially during the writing process, was very helpful. I would also like to thank my second supervisor, Prof. Virginia Weis, for the very productive collaboration in her lab and all her input and enthusiasm which helped me to put across my findings and ideas with much more structure and clarity.

Many thanks go out to the entire Davy Lab group, specifically to Clint Oakley who has always been there to give me advice and support when I needed it. A special thanks goes to Shaun Wilkinson who took the time to teach me the molecular techniques I needed and was always ready to answer my questions. I would also like to thank the SBS staff and technical team for all their assistance.

I would like to thank my friends back home (specifically Hadas, Inbal and Yulia) for all their emotional support whenever I needed it through all media channels known to mankind. You guys made me feel like you live just around the corner. To my new friends I acquired over this time, especially Julia - thanks for all the emotional support, talks, coffees, drinks, trips, movies and so much more. Without you I don't think I could have lasted this long! A massive thanks to my wonderful partner Richard, who found me broken (literally) and was there for me through a very challenging year. Thanks for all the times you made me smile after a frustrated day in the lab, for all the dinners you cooked for me and the flowers you gave me. But most importantly, thanks for all your listening and understanding; I will always be grateful.

Finally, I wish to thank my family, especially to mom, for her endless love and support along the way and for her patience and encouragement. 


\section{List of Figures}

Figure 1.1 Symbiosis establishment and persistence phases in cnidariandinoflagellate symbiosis.

Figure 1.2 Regulation of the symbiont population in alga-invertebrate symbioses

Figure 1.3 The phylogenetic relationships between Symbiodinium clades

Figure 1.4 Illustration of the mutualistic to parasitic continuum

Figure 1.5 Bleached coral, Acropora sp.

Figure 1.6 Schematic diagram illustrating the two processes that can account for changes in the Symbiodinium population of a cnidarian host.

Figure 1.7 Aiptasia sea anemone as a model system

Figure 2.1 Colonisation success in host Aiptasia varied with symbiont identity

Figure 2.2 Spatial pattern of symbiont colonisation in Aiptasia is similar for different symbionts

Figure 2.3 Gross photosynthesis and dark respiration of the holobiont varied with symbiont identity

Figure 2.4 Host performance varied with symbiont identity 
Figure 3.2 Symbiont success in simultaneous competitive exposure varied with Symbiodinium species and temperature

Figure 3.3 Symbiont specificity is the key factor in determining symbiont success in delayed competitive exposure

Figure 4.1 Positive and negative controls of caspase-like enzyme activity in aposymbiotic specimens of the sea anemone Aiptasia

Figure 4.2 Colonisation success in Aiptasia varied with symbiont type 78

Figure 4.3 Caspase activity differed with symbiont type 80

Figure 4.4 Symbiont density has no effect on caspase activity in Aiptasia

Figure 5.1 Optimisation of the flow cytometry method

Figure 5.2 Flow cytometry analysis of cell cycle progression by homologous and heterologous symbionts in Aiptasia

Figure 5.3 Pattern of cell cycle progression was similar between different symbiont species

Figure 6.1 The "winnowing" process: mechanisms of symbiont selection and regulation in the cnidarian-dinoflagellate symbiosis as highlighted by this thesis

Figure A3.1 Quantitative PCR standard curve

Figure A4.1 Thermal bleaching in host Aiptasia containing mixture of symbiont species

Figure A4.2 Symbiont success in mixed populations varied with Symbiodinium species and temperature 156 
Figure A5.1 Fv/Fm as a proxy for algae health in hospite was quantified for 23 days after inoculation

Figure A6.1 Flow cytometry analysis of cell cycle progression by homologous and heterologous symbionts in Aiptasia one week after inoculation

Figure A6.2 Flow cytometry analysis of cell cycle progression by homologous and heterologous symbionts in Aiptasia two weeks after inoculation

Figure A6.3 Flow cytometry analysis of cell cycle progression by homologous and heterologous symbionts in Aiptasia four weeks after inoculation

Figure A6.4 Optimisation of the flow cytometry method. Measurements of algae lost during the washing protocol from each of the three washes used 
List of Tables

\section{List of Tables}

Table 2.1 Identity and geographical origin of Symbiodinium cultures and the original host species if known

Table 3.1 Clade-specific primers: sequences, $T m$ values and amplicon sizes 61

Table A3.1 Number of ITS2 copies calculated for each Symbiodinium species using quantitative PCR 


\section{Table of Contents}

Abstract.................................................................... III

Contributions and Publications............................................... V

Acknowledgments......................................................... VII

List of Figures.................................................................................................... VIII

List of Tables................................................................... XI

Chapter 1:

General Introduction........................................................... 17

\begin{tabular}{|c|c|}
\hline 1.1 & Symbiosis...... \\
\hline 1.2 & Cnidarian - Dinoflagellate Symbiosis.............. \\
\hline 1.2.1 & Symbiosis architecture.... \\
\hline 1.2 .2 & Symbiosis function and carbon translocation... \\
\hline 1.2 .3 & Nitrogen and phosphorus recycling..... \\
\hline 1.2 .4 & Symbiont uptake and proliferation.... \\
\hline 1.2 .5 & Crosstalk, and regulation of host and symbiont biomass......... \\
\hline 1.2 .6 & Regulation of symbiont cell growth and proliferation.......... \\
\hline 1.2 .7 & Symbiont diversity ....................... \\
\hline 1.3 & Coevolution between Symbiodinium and its cnidarian hosts.... \\
\hline 1.4 & Climate change and coral bleaching... \\
\hline 1.5 & The adaptive bleaching theory.. \\
\hline 1.6 & Aiptasia as a model system... \\
\hline 7 & Aim and specific objectives.. \\
\hline
\end{tabular}

Chapter 2:

Symbiont identity influences patterns of symbiosis establishment, host growth and asexual reproduction in a model cnidarian-dinoflagellate symbiosis.................. 38

$2.1 \quad$ Introduction............................................ 38

2.2 Materials and Methods................................... 40

2.2.1 Experimental organisms.............................. 40

2.2.2 Inoculation of aposymbiotic anemones with symbionts.............................................. 41

2.2.3 Symbiont proliferation................................. 41

2.2.3.1 Spatial pattern of colonisation........................... 41 
2.2.3.2 Quantification of symbionts during colonisation.............. 42

2.2.4 Photo-physiology and host performance................... 42

2.2.4.1 Photosynthesis and respiration of the host-symbiont partnership.......................................... 42

2.2.4.2 Host growth and asexual reproduction..................... 43

2.2.5 DNA sequencing........................................ 43

2.2.6 Statistical analysis..................................... 44

$2.3 \quad$ Results.................................................. 44

2.3.1 Symbiont proliferation in hosts............................ 45

2.3.2 Photo-physiology and hosts performance.................... 48

2.3.2.1 Photosynthesis and respiration of the host-symbiont partnership.......................................... 48

2.3.2.2 Host growth and asexual reproduction...................... 49

$2.4 \quad$ Discussion........................................... 51

2.4.1 Colonisation success varies with symbiont identity........... 51

2.4.2 Host photo-physiology and performance..................... 53

Chapter 3:

Competitive symbiont uptake in a model cnidarian-dinoflagellate symbiosis under heat stress: specificity trumps acquisition of thermo tolerant symbionts.................. 55

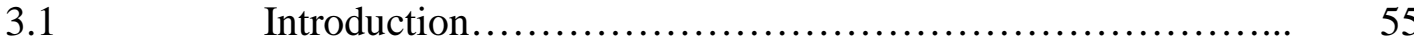

3.2 Materials and Methods..................................... 57

3.2.1 Experimental organisms................................. 57

3.2.2 Simultaneous competitive exposure...................... 58

3.2.3 Delayed competitive exposure............................ 59

3.2.4 Quantitative PCR...................................... 59

3.2.5 Statistical analysis..................................... 61

$3.3 \quad$ Results................................................. 62

3.3.1 Temperature-induced bleaching and controls............... 62

3.3.2 Simultaneous competitive exposure........................ 63

3.3.3 Delayed competitive exposure............................. 63

$3.4 \quad$ Discussion............................................... 66

3.4.1 Simultaneous competitive exposure....................... 66

3.4.2 Delayed competitive exposure........................... 67 
3.4.3 Ecological implications

Chapter 4:

Host cell apoptosis and the regulation of different symbiont species in the cnidariandinoflagellate symbiosis............................................... 70

4.1 Introduction........................................ 70

4.2 Materials and Methods................................. 72

4.2.1 Experimental organisms.............................. 72

4.2.2 Inoculation of aposymbiotic sea anemones with symbionts.............................................. 73

4.2.3 Real-time visualisation of caspase synthesis in Aiptasia....... 73

4.2.4 Preparation of tissue homogenate and quantification of

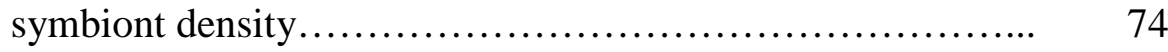

4.2.5 Assessment of host caspase-like enzyme activity in Aiptasia............................................. 74

4.2.6 DNA sequencing....................................... 76

4.2.7 Statistical analysis....................................... 77

$4.3 \quad$ Results..................................................... 77

4.3.1 Colonisation success.................................. 77

4.3.2 Caspase-like enzyme activity ........................... 79

4.3.3 Correlation between caspase activity and algal density........ 79

$4.4 \quad$ Discussion.............................................. 81

4.4.1 Temporal pattern of apoptosis during host colonisation............................................. $\quad 82$

4.4.2 Differential induction of apoptosis and its role in hostsymbiont specificity................................... 83

Chapter 5:

Symbiont cell cycle progression is unaffected by symbiont identity in a model cnidarian-dinoflagellate symbiosis...

Materials and Methods.

5.2.1 Experimental organisms............................ 87

5.2.2 Inoculation of aposymbiotic sea anemones with symbionts............................................ 88 
5.2.3 Sample preparation...................................... 88

5.2.4 Cell cycle measurement and analysis........................ 89

5.2.5 DNA sequencing...................................... 91

5.2.6 Statistical analysis......................................... 91

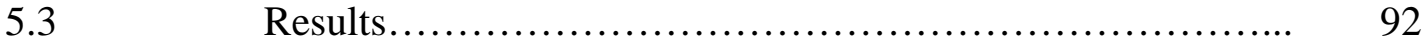

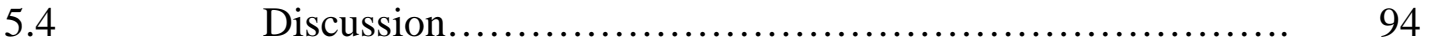

Chapter 6:

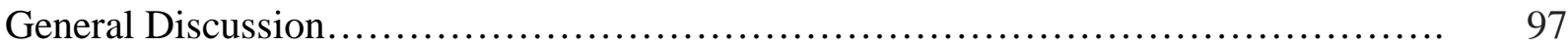

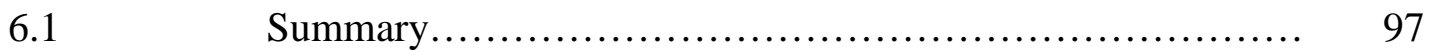

6.2 The relative importance of post-phagocytosis control mechanisms.............................................. 98

6.3 Will corals be able to adapt to climate change?...................... 103

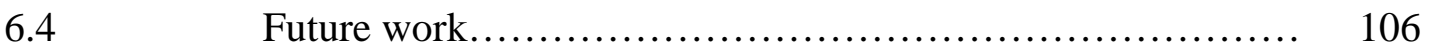

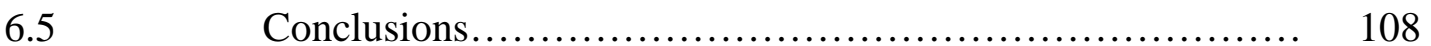

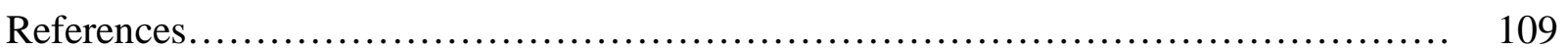

Appendix 1:

Symbiont identity influence patterns of symbiosis establishment, host growth, and asexual reproduction in a model cnidarian-dinoflagellate symbiosis..................... 138

Appendix 2:

ITS2 sequences of Symbiodinium species used in this study

Appendix 3:

Calculation of ITS2 copies for Symbiodinium species using quantitative PCR

Appendix 4:

Simultaneous competitive exposure in a model cnidarian-dinoflagellate symbiosis under heat stress. 
Table of Contents

Appendix 5:

Measuring $\mathrm{F}_{\mathrm{v}} / \mathrm{F}_{\mathrm{m}}$ as a proxy for a proxy for algae health in hospite under thermal stress

A5.1

Materials and Methods....

Appendix 6:

Flow cytometry analysis of cell cycle progression. 


\section{Chapter 1}

\section{General Introduction}

\subsection{Symbiosis}

de Bary (1879) defined symbiosis as the "living together of two differently named organisms". Today symbiosis is defined as an intimate, long-term relationship between individuals of different species (Goff, 1982), and allows for the fact that these associations are dynamic in time and space, and may influence one or both partners (Bronstein, 1994). A symbiotic organism may live inside its host (endosymbiosis) or outside it (ectosymbiosis), and it may depend exclusively upon the symbiotic relationship for survival (obligate symbiosis) or be able to exist in a free-living state (facultative symbiosis) (Sheppard et al., 2009). Although symbiotic relationships can be divided into three broad types: (a) mutualism, where both partners derive benefit from the relationship, though not necessarily to the same degree, such as gut flora which are involved in breaking down cellulose and related substances (Howard, 1967), and nitrogen fixation by Rhizobium bacteria in root nodules of leguminous plants (Beringer et al., 1979; Dimijian, 2000); (b) commensalism, where one partner benefits and the other is unaffected. For example, bacteria colonising the mouth or skin of a healthy person; and (c) parasitism, where one partner benefits from the relationship at the expense of the other, such as intestinal worms and herpes viruses (Dimijian, 2000). However, many symbioses do not fit neatly into a single category (Douglas, 1994), so symbiosis is better described as a continuum that spans from parasitism to mutualism (Dimijian, 2000), with the ability to shift along the continuum from one end to the other (Ewald, 1987; Thompson, 1988; Bronstein, 1994).

Symbiotic relationships are very common in nature; they can occur between closely related species, as well as between distantly related organisms such as bacteria and eukaryotes. The relationships comprise all degrees of intimacy, from closely integrated intracellular symbioses to looser external associations between organisms (Herre et al., 1999; Dimijian, 
2000; Douglas, 2008). Symbiotic relationships are common in marine environments in both tropical and temperate waters (Roughgarden, 1975). For example, bacterial symbionts provide light emission for a number of host organisms such as squid (Hastings, 1983; Widder, 2010; Hellinger et al., 2017), sea anemones provide protection to clownfishes and gain waste nutrients in return (Fautin, 1991; Schmiege et al., 2017), and intracellular chemoautotrophic symbionts enable sulphide detoxification in bivalves (Joyner et al., 2003). Bacterial populations in sponges comprise up to $40 \%$ of sponge volume, with high microbial diversity and novel species that have not been found in other ecosystems (Taylor et al., 2007). These microbial populations have a profound impact on host biology, with photosynthetically fixed carbon from cyanobacterial symbionts, for instance, providing > $50 \%$ of the energy requirements of certain tropical sponges (Wilkinson, 1983), and other microbial associates contributing to host defences via the production of biologically active metabolites (Unson et al., 1994; Schmidt et al., 2000; Webster and Taylor, 2012).

\subsection{Cnidarian - Dinoflagellate Symbiosis}

Among the most significant marine mutualisms are those found between cnidarians and their photosynthetic dinoflagellate symbionts (Roth M. S, 2014). These interactions, in particular between anthozoan cnidarians and dinoflagellates of the genus Symbiodinium, underpin the existence and success of coral reef ecosystems in nutrient poor environments (Little et al., 2004; Wang et al., 2012; Lesser et al., 2013). Cnidarian-dinoflagellate symbiosis is found in both temperate and subtropical environments, but has the most ecological importance on tropical reefs (Muller-Parker and Davy, 2001; Wicks et al., 2010).

\subsubsection{Symbiosis architecture}

The symbiotic dinoflagellates usually exist inside the cells of the host cnidarian's gastrodermis, where they are enclosed by a membrane complex comprised of a series of membranes of algal origin surrounded by an outer, host-derived membrane; together, this structure is referred to as the symbiosome membrane complex (Wakefield et al., 2000; Yellowlees et al., 2008; Davy et al., 2012). The symbiosome membrane complex is critical to 
metabolic interactions between the symbionts and their host, since all nutrient trafficking between the partners must occur through its membranes (Yellowlees et al., 2008). A study by Peng et al., (2010) examined the protein components of the symbiosome membrane (i.e. the outer, host-derived membrane) and found 17 different proteins that act as receptors and transporters, as well as in ATP synthesis and as anti-apoptosis proteins. Little else is known about this membrane. Another family of proteins named Niemann-Pick type C (NPC) was recently found in the vicinity of the symbiosome membrane and might have a putative function in the symbiosis (Dani et al., 2017); for example NPC1 is well characterised in humans since this protein mediates intracellular cholesterol trafficking (Vance, 2010). Other studies have shown that NPC1 has an important role in the entry of several viruses into human cells through cell-surface binding (Dahlmann et al., 2015), which might mean that NPC1 is involved in recognition of the symbiont cell-surface glycome (Logan et al., 2010a).

\subsubsection{Symbiosis function and carbon translocation}

Photosynthesis is the most important feature of the cnidarian-dinoflagellate symbiosis, as photosynthetically fixed carbon is translocated to the host, supporting its respiration, growth and reproduction (Muscatine, 1990). Furthermore, the symbiotic dinoflagellates promote calcification of stony corals, through a process known as 'light enhanced calcification' (Kawaguti and Sakumoto, 1948; Pearse and Muscatine, 1971; Gattuso et al., 1999; Tentori and Allemand, 2006). In return, the cnidarian host provides inorganic carbon, nitrogen and phosphorus, a high-light environment and refuge from herbivory to the symbiotic dinoflagellate (Furla et al., 2005). In tropical environments, the cnidarian-dinoflagellate symbioses is mostly autotrophic with respect to carbon (Muscatine et al., 1984). However, in temperate environments the amount of carbon translocated to the host is generally insufficient for supporting host metabolic demands and therefore the host must acquire additional carbon from heterotrophic sources such as predation and uptake of particulate and dissolved organic material from the environment (Lewis and Price, 1975; Muller-Parker and Davy, 2001). There is still much uncertainty about the compounds that are translocated to the host (reviewed in Davy et al. 2012). Muscatine and Hand (1958) were the first to demonstrate photosynthate translocation, in the symbiotic temperate sea anemone Anthopleura elegantissima. Later, a study by Muscatine (1967) measured the released photosynthetic 
products from Symbiodinium by incubating freshly isolated Symbiodinium cells in homogenized host tissue. These and several other studies have used ${ }^{14} \mathrm{C}$-bicarbonate labelling, measuring the proportion of label found in the host's tissues to quantify the percentage of fixed carbon translocated. Using this method, it has been estimated that symbiotic dinoflagellates translocate $5-60 \%$ of their photosynthetically fixed carbon across a wide range of cnidarian-dinoflagellate symbioses (Muscatine and Cernichiari, 1969; Trench, 1971a; Hofmann and Kremer, 1981; Clayton and Lasker, 1984; Farrant et al., 1987; Stambler and Dubinsky, 1987; Davy et al., 1997a). Muscatine et al., (1983) later introduced an alternative method for quantifying translocation based upon the assumption that the algae transfer all photosynthetically-fixed carbon not used for their growth or respiration; using this method, it has been estimated that more than $90 \%$, and sometimes as much as $99 \%$, of photosynthate is released to the host (Muscatine et al., 1984; Steen and Muscatine, 1984; Davy et al., 1996).

Despite these approaches, it is still not entirely clear how much photosynthetic carbon is translocated to the host, or in what forms the carbon is translocated (Davy et al., 2012). Muscatine (1967) was the first to isolate symbiotic dinoflagellates from corals and giant clams and incubate these in homogenised host tissue, revealing that glycerol (up to $90 \%$ of the total carbon released) was the major product released. Since then, several studies have also found glycerol to be the main compound released by isolated symbionts, with other metabolites such as glucose, the amino acid alanine, and organic acids such as fumarate, succinate, malate, citrate and glycolate being released in smaller amounts (von Holt and von Holt, 1968; Trench, 1971a; b, 1974; Sutton and Hoegh-Guldberg, 1990). In addition to these compounds, studies with dinoflagellates isolated from the coral S. pistillata and the sea anemone Condylactis gigantea led to reports of fat droplets (i.e. lipids) being released from the cells (Kellogg and Patton, 1983; Patton and Burris, 1983; Muscatine et al., 1994; Peng et $a l ., 2011)$. However, isolated dinoflagellates might behave differently from when they are in symbiosis (e.g. Sutton and Hoegh-Guldberg 1990), while the potentially rapid catabolism of translocated compounds in the host's tissue can confound the identification of translocated compounds in hospite (Davy et al., 2012). However, methodological advances, especially in the field of metabolomics, have facilitated this identification, suggesting that glucose is in fact the major compound translocated, while glycerol release from isolated algae is probably stimulated by osmotic stress (Whitehead and Douglas, 2003; Burriesci et al., 2012). Several 
recent studies have confirmed this conclusion and identified a range of other more minor mobile compounds (Tremblay et al., 2014; Hillyer et al., 2016, 2017; Sogin et al., 2016), however further work is still necessary to understand the role of these various metabolites in the symbiosis.

\subsubsection{Nitrogen and phosphorus recycling}

The coral holobiont has the ability to take up and recycle important nutrients such as nitrogen and phosphate, which enable corals to live in a low-nutrient environment (Rädecker et al., 2015). These nutrients are important components in many biological molecules and mechanisms, and potentially play an important role in controlling the algal cell cycle (see later). Different forms of nitrogen can be acquired from different sources, such as ammonium from the host's catabolic processes (Cates and McLaughlin, 1976; Rahav et al., 1989) and dissolved inorganic nitrogen (DIN) from the environment (Muscatine and D'Elia, 1978; Grover et al., 2002; Badgley et al., 2006). Although both the coral host and the dinoflagellate symbionts have the ability to assimilate DIN, the algae are thought to be the primary site of this uptake, with the main forms of DIN assimilated being ammonium $\left(\mathrm{NH}_{4}{ }^{+}\right)$and nitrate $\left(\mathrm{NO}_{3}{ }^{-}\right)$(Grover et al., 2002; Pernice et al., 2012). The nitrogen assimilated, from the host or from the environment, can either be used for algal metabolism, stored by the algae or translocated to the host as organic nitrogenous compounds such as amino acids, which may then be used again in host metabolism; this creates a very efficient means of recycling nitrogen within the holobiont (Wang and Douglas, 1999; Reynaud et al., 2009; Kopp et al., 2013; Rädecker et al., 2015). In addition to nitrogen cycling, nitrogen conservation is an important aspect of the cnidarian-dinoflagellate symbiosis. Here, the host preferentially uses translocated carbon from the symbionts for its metabolic needs, so preserving nitrogenous compounds such as amino acids that may otherwise be catabolised. As with nitrogen recycling, this conservation facilitates the survival of corals in nutrient-poor waters (Wang and Douglas, 1999).

Phosphate flux in the cnidarian-dinoflagellate symbiosis is less well understood. The holobiont can take up dissolved organic and inorganic phosphorus from the environment (Muller-Parker et al., 1990). In the past, the uptake of phosphate was thought to primarily be 
driven by the symbiont, since aposymbiotic (i.e. symbiont-free) hosts are not able to take up $\mathrm{PO}_{4}{ }^{-3}$ (Muller-Parker et al., 1990), while isolated dinoflagellates are able to do so (D'Elia et al., 1983) and the uptake is light sensitive (Sorokin, 1992). It was suggested that this phosphate uptake is enabled by transporters located exclusively in/on the symbionts (D'Elia, 1977). However, a more recent study showed that active transport is conducted by both the host and symbionts, enabling both partners to take up phosphate against a concentration gradient from the environment to the cells (Godinot et al., 2009).

Further study is still needed to better understand nutrient fluxes in the cnidariandinoflagellate symbiosis, however it is clear that these fluxes are likely to be tightly controlled (Davy et al., 2012). This control may enable the host to limit nutrient supply to its symbionts, thus providing a mechanism by which host can regulate its algal population density (see later) (Jackson and Yellowlees, 1990; Weis, 1993; Cook et al., 1994).

\subsubsection{Symbiont uptake and proliferation}

There are two ways for a host to acquire symbionts: (1) vertically, where the symbiont is passed directly from host parent to offspring, and occurs within the confines of the host's sexual cycle; or (2) horizontally, where host sexual progeny must acquire symbionts from the environment. The establishment of the symbiosis may depend upon a number of algal properties and interactions with the host cell (McAuley and Smith, 1982). One of the first mechanisms to establish a symbiosis is host-symbiont recognition, which includes a cascade of molecular signalling that occurs between the host cnidarian and the algae destined to be symbionts (Fig. 1.1). These steps have been referred to as 'the winnowing' (Nyholm and McFall-Ngai, 2004) and are necessary to establish a successful, specific symbiosis. Among the most studied mechanisms involved in inter-partner recognition are lectin/glycan interactions (Fujita, 2002; Fujita et al., 2004; Logan et al., 2010a). In the well-studied squidluminous Vibrio fischeri symbiosis, the squid produces a mucus containing mannose glycans which are bound by lectins from the symbiont and only $V$. fischeri is able to move past the mucus to the next winnowing step (Nyholm and McFall-Ngai, 2004). Lectin/glycan interactions have also been implicated as an inter-partner signalling mechanism during the onset of symbiosis in the plant-nitrogen-fixing Rhizobium association (D'Haeze and Holsters, 
2002). The use of the same lectin/glycan interactions for recognition in two very different symbioses suggests that these interactions may be a common mechanism of recognition in other horizontally transmitted partnerships. Indeed, a more recent examination of the mechanism of recognition in the cnidarian-dinoflagellate symbiosis showed a winnowing process analogous to that in well-described model symbioses (Wood-Charlson et al., 2006). These interactions were first suggested 30 years ago in the Hydra viridis-chlorophyte symbiosis (e.g. Meints and Pardy, 1980) and more recently in anthozoan-Symbiodinium associations (Lin et al., 2000). It has been shown that the presence of the lectin concanavalin A (ConA), which binds to $\alpha$-mannose/ $\alpha$-glucose, can inhibit repopulation of bleached adult anemones by Symbiodinium. The same result occurs when symbionts are treated with enzymes that alter their cell surface (Lin et al., 2000; Wood-Charlson et al., 2006). This molecular signalling is also likely to participate in the regulation of stability and breakdown of the symbiosis (Trench, 1997; Reynolds et al., 2000; Nyholm and McFall-Ngai, 2004; Weis et al., 2008; Davy et al., 2012).

1. Initial contact

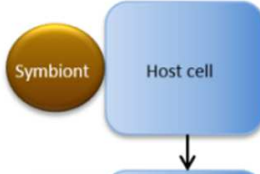

2. Engulfment

3. Sorting

4. Proliferation

5. Dynamic stability

6. Dysfunction
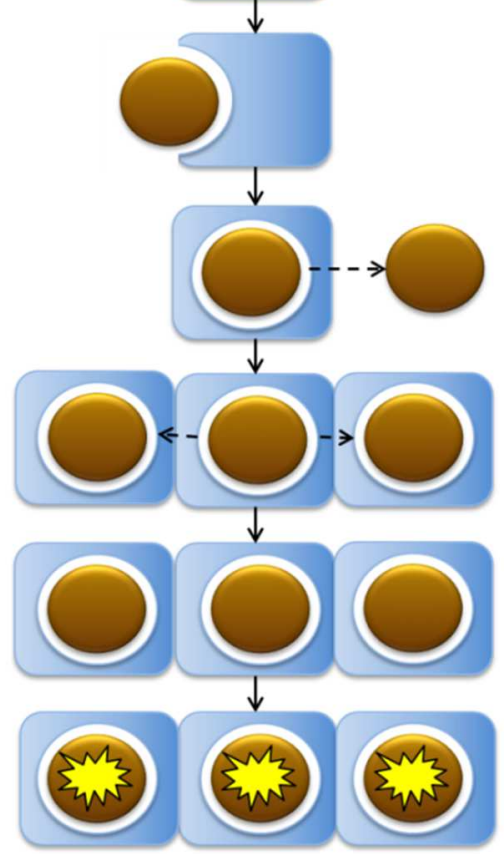

Fig. 1.1. Symbiosis establishment and persistence phases in cnidariandinoflagellate symbiosis. (1) Initial contact between the algal symbiont and the cnidarian host cell; (2) engulfment of the symbiont by the host cell; (3) sorting of the symbiont inside the host cell; (4) cell division and proliferation of the symbiont inside the host tissue; (5) dynamic stability, where the symbiont population is maintained at a steady density; and (6) symbiosis dysfunction and the breakdown of the symbiosis as a response to internal or external stress, such as high temperature and radiation (redrawn from Davy et al., 2012). 
After successful recognition, the algae enter the host cell (Fig. 1.1). The general agreement is that the symbionts enter the host cell through phagocytosis by the endodermal cells, soon after the development of the larval mouth. However, Marlow and Martindale (2007) found symbionts in the endoderm and ectoderm of $F$. scutaria embryos prior to the formation of a mouth, highlighting that uptake may potentially occur earlier during development. Symbionts transferred through vertical transmission have also been observed in oocytes, zygotes, ectoderm of embryos and the epidermis of planulae (Benayahu et al., 1992; Benayahu and Schleyer, 1998; Hirose et al., 2000, 2001; Davy and Turner, 2003). The mechanism of symbiont sequestration into germ cells or developing embryos has been studied in several soft corals and it has been suggested that, during oogenesis, temporary gaps open in the mesogloea underlying the follicular cells. Symbionts within vacuoles are translocated through these gaps and accumulate at the perioocytic zone, located between the mesogloea and oocytic microvilli. Prior to the beginning of the breeding season, symbionts pass through the plasma membrane of the oocyte and rest inside the periphery of the oocytes (Benayahu et al., 1992; Benayahu and Schleyer, 1998).

How does the alga manage to avoid intracellular attack during its invasion of the host cell? Unlike phagosomes containing food particles, or dead or impaired algae, the phagosomes containing live and functional algae do not show any signs of lysosomal fusion (Fitt and Trench, 1983). Therefore, it has been suggested that Symbiodinium cells can survive in symbiosomes, in part by manipulating the cnidarian's innate immune signalling pathways (Karakashian and Rudzinska, 1981; Chen et al., 2004, 2005; Hong et al., 2009; Venn et al., 2009; Neubauer et al., 2017). For example, studies of the anemone Aiptasia pulchella (= Exaiptasia pallida) showed that the presence of Symbiodinium cells in the phagosomes can change the maturation of the phagosome and endosomal trafficking (Chen et al., 2003, 2004). Indeed, it is now well known that negative and beneficial interactions share many of the same host-symbiont signalling pathways and cellular responses (Davy et al., 2012).

\subsubsection{Crosstalk, and regulation of host and symbiont biomass}

Once the symbiosis has been established, its persistence requires the coordination of both host cell and symbiont proliferation (Davy et al., 2012; Fig. 1.2). Without this regulation, 
symbiont density might be too low and therefore might not be able to support the host's metabolic demands (Muscatine and Neckelmann, 1983; Taylor et al., 1989), while excessive symbiont replication might cause the symbionts to overgrow the host's tissues (Sachs and Wilcox, 2006a; Stat et al., 2008). Four potential mechanisms have been suggested for the regulation of the symbiont population in alga-invertebrate symbioses: (1) Degradation of surplus symbionts by the host cell (Fig. 1.2A), which appears to be an intracellular process regulated by the host's gastrodermal cells. Titlyanov et al., (1996) showed that, in eight different stony coral species, the proportion of degraded symbionts is between 1 to $6 \%$ per day, a value similar to the symbiont division rate. However, the mechanism and its importance in the regulation of the symbiont population are still unclear. (2) Expulsion of surplus symbionts via active exocytosis or by detachment of the host cell (Fig. 1.2A). There are a wide range of expulsion rates in different host species, suggesting that the importance of this mechanism varies between different partners. Low rates of symbiont expulsion (0.1-1\% symbionts per day) were found in several tropical cnidarians such as the stony corals Stylophora pistillata and Pocillopora damicornis (Hoegh-Guldberg et al., 1987; HoeghGuldberg and Smith, 1989a; Stimson and Kinzie, 1991), as well as in the soft corals Xenia macrospiculata and Heteroxenia fuscescens (Hoegh-Guldberg et al., 1987). However, in the temperate sea anemone Anthopleura elegantissima, expulsion can sometimes exceed the daily growth rate of the symbionts and is considered to be the primary process for maintaining symbiont density (McCloskey et al., 1996). Baghdasarian and Muscatine (2000) reported that the sea anemone Aiptasia pulchella had a daily expulsion rate of $4.6 \%$ of its symbionts and concluded that expulsion is one of the primary mechanisms that regulate symbiont density in some cnidarian-dinoflagellate symbioses. (3) Host cell apoptosis, a highly conserved form of programmed cell death (Raff, 1998; Fig. 1.2A). This process is characterized by a precise, fixed set of events that ultimately leads to cell death. Once the cascade is initiated, the cells go through morphological changes such as cell shrinkage, fragmentation of DNA, condensation of chromatin, and the formation of apoptotic bodies that contain cellular debris (Weis, 2008). Dunn and Weis (2009) studied the role of host cell apoptosis in the post-phagocytic winnowing process. They challenged Fungia scutaria larvae with different algal types and tested for caspase activity, a key component of apoptosis. They found that different algal types displayed different amounts of caspase activation that corresponded with infection success in the host larvae. The entry of heterologous symbionts (i.e. symbionts not normally found in a particular host) into host cells resulted in host cell apoptosis as a means of removing inappropriate symbiont cells, while hosts challenged with 
homologous symbionts (i.e. the usual symbionts) did not exhibit the same amount of apoptotic activity. (4) Regulation of the symbiont cell cycle, and coordination with host cell proliferation (Muscatine and Pool, 1979; Weis, 2008; Davy et al., 2012). This mechanism will be discussed in the next section.

A

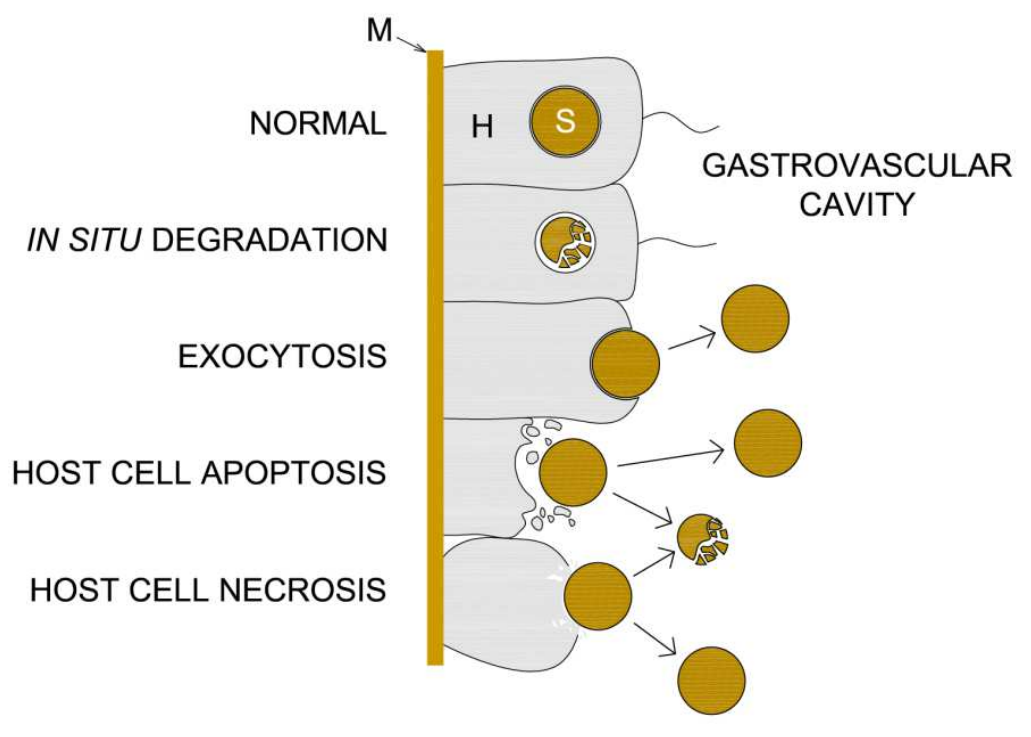

Fig. 1.2. Regulation of the symbiont population in algainvertebrate symbioses.

(A) Symbiont (S) loss via degradation within their host cell $(\mathrm{H})$ or expulsion of symbiont cells via active exocytosis. Host cells have also been observed undergoing apoptosis, where they shrink and forms multiple apoptotic bodies, and release symbionts into the gastrovascular cavity (redrawn from Gates et al., 1992); (B) Control of progression through the symbiont cell cycle by the host. $\mathrm{G}_{0}, \mathrm{G}_{1}, \mathrm{~S}, \mathrm{G}_{2}$ and $\mathrm{M}$ are the phases of the eukaryotic cell cycle (see text) (redrawn from Alberts et al., 2002). 


\subsubsection{Regulation of symbiont cell growth and proliferation}

In order to understand how the host might regulate symbiont growth and proliferation, it is necessary to understand the eukaryotic cell cycle. The most basic function of the cell cycle is to duplicate accurately the DNA and then separate the two copies precisely into two genetically identical daughter cells (Alberts et al., 2002). This process is highly complex and conserved during evolution, yet it is still not completely understood. The cell cycle is comprised of two main phases: the $\mathrm{S}$ (synthesis) phase, during which the DNA is replicated, and the M (mitosis) phase, during which mitosis (nuclear and cytoplasmic division) occurs, followed by cell division (Fig. 1.2B). Another two phases, known as gap (G) phases, are generally located between the $S$ and $M$ phases (i.e. $G_{1}$ and $G_{2}$ ). The main purpose of these gap phases is to provide time for the cell to monitor the internal and external environment to ensure that the conditions are suitable prior to any investment in DNA replication and cell division (Alberts et al., 2002). Cells in $\mathrm{G}_{1}$ may also enter a nonproliferative state $\left(\mathrm{G}_{0}\right)$ and may stay there for long periods or even indefinitely. The cell cycle always progresses in the same order and is irreversible. The time and order of cell cycle progression is regulated by a complex control system and "checkpoints," which can pause the cycle if there is a problem with the previous stage (Alberts et al., 2002, 2013; Davy et al., 2012).

The host could regulate the symbiont cell cycle in a number of ways:

(1) Nutrient regulation. Symbiotic dinoflagellates are often limited with respect to nitrogen and phosphate supply from the host, as evidenced by their enhanced growth rate when supplied with an exogenous source of dissolved nitrogen or phosphorus (Cook and D'elia, 1987; Muscatine et al., 1989; Hoegh-Guldberg and Smith, 1989b; Cook et al., 1994; McAuley and Cook, 1994; Koop et al., 2001; Davy et al., 2006). These limitations could provide an explanation for the rapid growth of the dinoflagellates during the early stages of symbiosis establishment, when the dinoflagellates can grow as fast as they do in culture (Trench, 1971c; Schoenberg and Trench, 1980a; Berner et al., 1993). Moreover, the respiratory rate of freshly isolated Symbiodinium cells is lower than when they are in culture, providing further evidence that symbiont metabolism is inhibited by the host (Goiran et al., 1996). Given these observations, it has been suggested that inorganic nutrients and other metabolites play an important role in the control of the symbiont population in the cnidarian- 
dinoflagellate symbiosis. A study by Smith and Muscatine (1999) used the sea anemone Aiptasia pulchella and its clade B dinoflagellate symbiont to test whether the host actively regulates nutrients to its symbionts or whether nutrient limitation is simply a result of the host's nutritional status. They exposed anemones to a range of nutritional treatments and measured the symbiont population and growth rate. Starvation caused a reduction in growth rates and chlorophyll content, as well as an increase in the cellular $\mathrm{C} / \mathrm{N}$ ratio (for details about carbon translocation see above). When anemones were provided with dissolved inorganic nutrients, the $\mathrm{C} / \mathrm{N}$ ratio decreased rapidly, however similar recovery was not seen with respect to population growth, suggesting that other factors, rather than inorganic nutrients, limit symbiont growth in hospite. The authors also used flow cytometry to examine the dinoflagellate cell size and DNA content under different nutritional conditions. They showed that the $\mathrm{G}_{1}$ phase is longer in the symbiotic state than in culture, while the other phases (i.e. $\mathrm{S}, \mathrm{G}_{2}$, and $\mathrm{M}$ phases) are shorter regardless of the symbiotic state. This suggests that the host is able to regulate symbiont proliferation by delaying the symbiont's progression through the $\mathrm{G}_{1}$ phase. They also noticed that nutrient supply has little effect on $\mathrm{G}_{1}$ duration, indicating that, while the symbiont population density may be regulated by nutrient availability in hospite, cell cycle progression of the symbiont is still restricted by some other host dependent factor(s). (2) Light is also an important inducer of symbiont cell division, since the carbon derived from photosynthesis is needed to support the metabolic demands of the cell cycle (Fitt, 2000). The light/dark regime has been shown to promote cell cycle progression from the $G_{1 / 2}$ phases to the next phase ( $S$ and $M$ phases, respectively), in a cultured clade $B$ Symbiodinium sp. from the coral Euphyllia glabrescens (Wang et al., 2008). Dark treatment was required for the mitotic division stage, while prolonged periods of light delayed cytokinesis and therefore created undivided cells. Wang et al. (2008) also found that different wavelengths of light had different effects on the Symbiodinium cell cycle, where red-light irradiation inhibits progression through the $G_{1}$ phase to $S$ phase to $G_{2} / M$ phase. This suggests that light might be another mechanism by which the host is able to control symbiont growth.

(3) Host cell division must be synchronized with its symbionts' proliferation to maintain a steady state. A study by Gladfelter (1983) studied the temporal pattern of mitosis in coral cells using the fluorescent nuclear stain DAPI (4',6'-diamidino-2- phenylindole). The study showed that the cells of Acropora cervicornis had a peak in mitotic index (MI) at midnight. This periodicity was suggested to have a link to feeding or photosynthesis. A study of the hydroid Myrionema amboinense showed that host cell division peaked at night, while its dinoflagellate division peaked at dawn (Fitt, 2000). This study showed that host cell division 
peaks at 12 to $24 \mathrm{~h}$ after feeding but that the symbiont cell cycle is set a further 12 to $24 \mathrm{~h}$ later (i.e., 24 to $36 \mathrm{~h}$ after feeding).

\subsubsection{Symbiont diversity}

Dinoflagellates are alveolate protists, consisting of 22,000 known species with a monophyletic origin closely related to ciliates, and with apicomplexan parasites as a sister

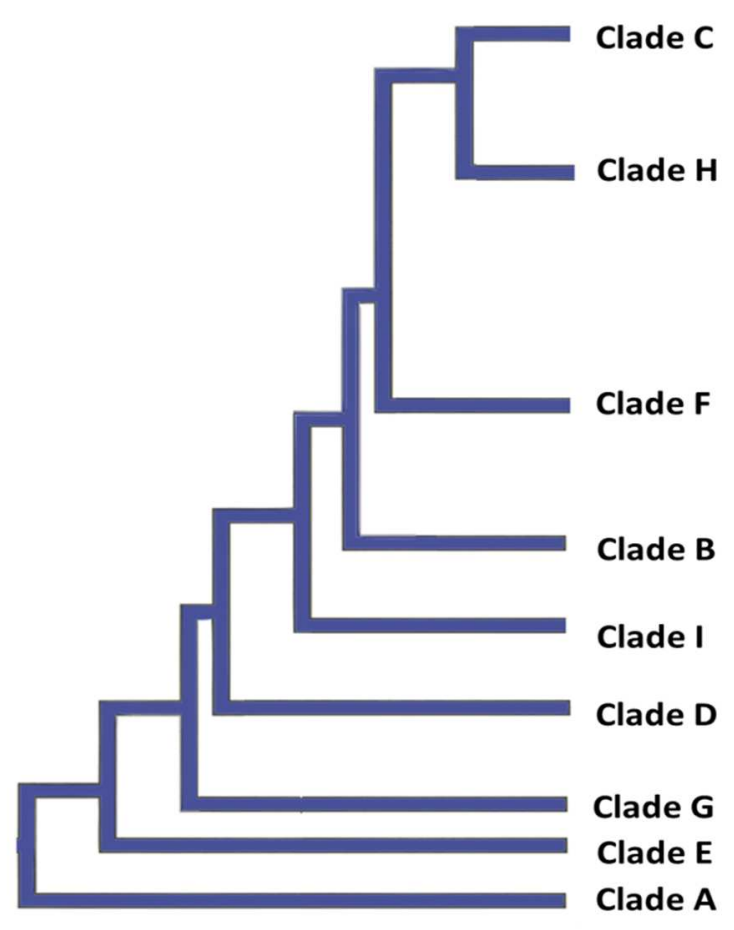

Fig. 1.3. The phylogenetic relationships between Symbiodinium clades (redrawn from Lesser et al., 2013). group (Lenaers et al., 1989; Stat et al., 2006). The genus Symbiodinium is the most common taxon of dinoflagellates found in symbiosis with marine invertebrates, including members of the Cnidaria, Platyhelminthes, Mollusca, Porifera, and Foraminifera (Trench, 1979; Stat et al., 2006). All members of the genus Symbiodinium have the same characteristic dominant phenotype, which under the light microscope appears as a brown coccoid cell of 5-15 $\mu \mathrm{m}$ diameter. This lack of morphological variation led early researchers to conclude that all symbiotic algae living with marine invertebrates belong to a single pandemic species, Symbiodinium microadriaticum (Freudenthal, 1962; Taylor, 1974). However, using multiple markers and molecular genetic approaches, we now know that this genus is much more diverse than originally thought, with nine clades (A-I; Fig. 1.3) and numerous sub-clades ('types') and species identified (LaJeunesse, 2002; Baker, 2003; Pochon and Gates, 2010; Stat et al., 2011). This genetic diversity is reflected in photoadaptative abilities (Chang et al., 1983), isoenzyme patterns (enzymes that differ in amino acid sequence but catalyze the same chemical reaction) (Schoenberg and Trench, 
1980b), cell motility patterns in culture (Fitt et al., 1981) and functional diversity (see later) (Iglesias-Prieto et al., 2004; Robison and Warner, 2006; Hennige et al., 2009). The symbiosis between invertebrates and dinoflagellates appears to be quite ancient. Most evidence points towards the mutualistic symbiosis between corals and symbiotic dinoflagellates originating in the mid Triassic (Trench, 1997). Clade phylogeny of Symbiodinium has identified clade A as the most ancestral, having originated 65-50 million years ago, and likely to be the first Symbiodinium group to infect a suitable host (Stat et al., 2008), with other clades diverging later during the Eocene to Miocene (Pochon et al. 2006; Fig. 1.3). Some members of clade A and $\mathrm{D}$ have been shown to have enhanced photo-protective capabilities resulting in significant tolerances of high solar irradiance and thermal stress (Banaszak et al., 2000; Reynolds et al., 2008; Ragni et al., 2010). However, they also show lower rates of carbon fixation and release to the host (Stat et al., 2008), and hosts that associate with clades A and D often exhibit lower overall fitness (Mieog et al., 2009; Leal et al., 2015). In contrast to clades A and D, clade C Symbiodinium can broadly be considered as the most beneficial symbionts for corals; they generally have higher rates of carbon fixation (Stat et al., 2008), translocate more carbon to the host, and enhance host growth more than members of clades A and D (Cantin et al., 2009; Mieog et al., 2009; Jones and Berkelmans, 2010; Leal et al., 2015). Clade $\mathrm{C}$ symbionts are also characterized by large sub-cladal diversity compared to clade A (Barneah et al., 2004). Each of this clade's genotypes exhibits a more specialized set of physiological capabilities, therefore increasing the adaptation to different environmental conditions (Barneah et al., 2004). Members of clade B also tend to be relatively beneficial to their host. For example, Starzak et al. (2014) showed that when the sea anemone Aiptasia sp. contains S. minutum (ITS2 B1; the type usually associated with this anemone), it benefits more than when in symbiosis with other symbiotic partners (Symbiodinium A1.4, S. voratum, and F5.1), with a higher rate of carbon translocation to the host.

Many coral species have been shown to host several different symbiont types (Iglesias-Prieto et al., 2004; Sampayo et al., 2007), while other coral species have been observed to host only one specific symbiont type (LaJeunesse et al., 2003). This specificity could make the coral susceptible to environmental change if the symbiont is stress-sensitive; on the other hand it could make the coral more resilient if the symbiont is stress tolerant (Sampayo et al., 2008; Stat et al., 2009; LaJeunesse et al., 2010). 


\subsection{Coevolution between Symbiodinium and its cnidarian hosts}

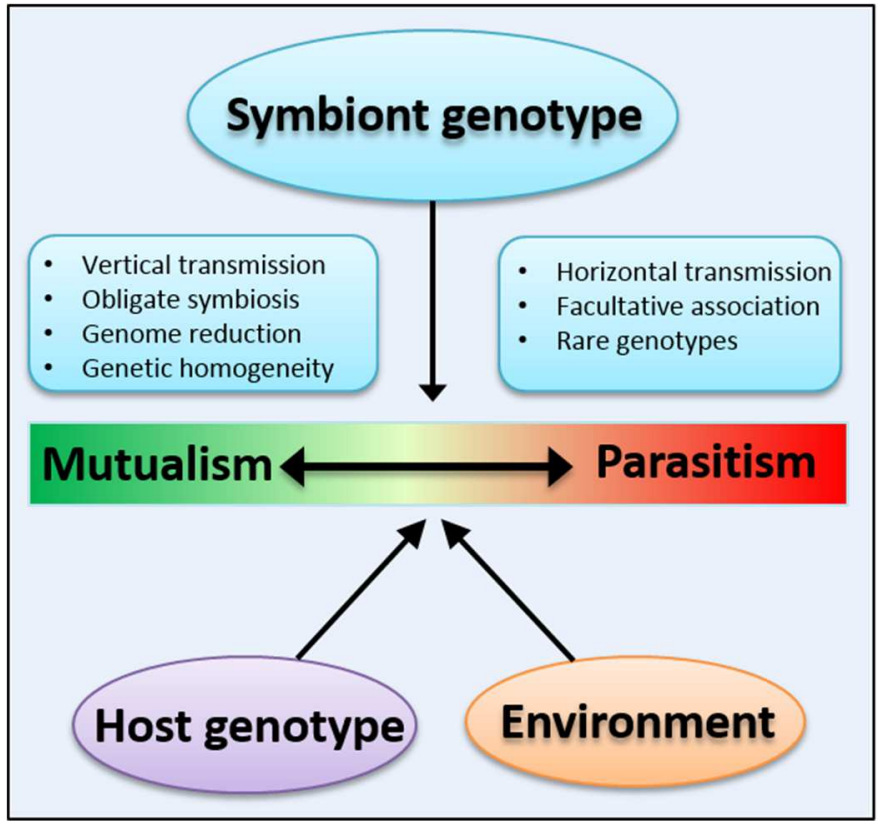

Fig. 1.4. Illustration of the mutualistic to parasitic continuum. Host and symbiont phylotype and the environment as primary drivers of the continuum, and the life-history characteristics of the symbiont (i.e., phenotypes) under selection (redrawn from Lesser et al., 2013).
The evolutionary success and survival of coral reefs will partly be determined by the composition of the corals' endosymbionts, since compositional differences in symbiont phylotype in the same host can lead to different outcomes for the host in similar environmental conditions (Lesser et al., 2013). Three factors regarding symbiont phylotype are considered as important steps towards successful mutualism (Fig. 1.4): (1) Vertical transmission (inherited from host parent to offspring) which favours increased metabolic integration, and leads to symbiont genome reduction and

obligate dependency for a specific host. Paradoxically, this type of mutualism is not common in nature; most broadcast spawning corals acquire symbionts horizontally, from the environment, in early ontogeny (Little et al., 2004; see above). Unlike vertical transmission, in which symbionts are inherited from host parent to offspring, horizontally transmitted symbionts are not tied to the reproduction of their host (Sachs and Wilcox, 2006a), which offers the host the opportunity to recombine with different algal types that are differentially adapted to the existing environmental conditions (Muller-Parker and D'Elia, 1997; Rowan, 1998). Virulence theory suggests, however, that horizontal transmission promotes the evolution of parasitism (Fine, 1975; Ewald, 1983; Bull, 1994); for example, the fitness of the jellyfish Cassiopea xamachana in symbiosis with clade A acquired by horizontal transmission, showed a shift toward parasitism and a reduction in host fitness, while the same 
clade A phylotype acquired by vertical transmission, produced a stable, higher fitness association (Sachs and Wilcox, 2006a). (2) Genetic uniformity of symbionts, which reduces the number of competitive phenotypes that a host has to cope with. (3) Little or no dependency of symbionts on a free-living state (Herre et al., 1999; Sachs et al., 2011a; Fig. 1.4). For example, clade A Symbiodinium types exhibit facultative lifestyles by maintaining members that are both free-living and symbiotic, and a propensity for parasitism (Stat et al., 2008).

Symbiont phylotype is not the only driver that can influence the symbiosis; the identity of the host has also been shown to determine the fitness outcomes of both partners and the stability of the symbiosis (Weis et al., 2001; Lesser et al., 2013). Moreover, changing conditions (biotic or abiotic) might create selection for opportunistic symbionts, which maximize their own fitness at the expense of their host, and therefore create a transition from mutualism toward parasitism (Thompson, 1988; Toft and Andersson, 2010; Sachs et al., 2011a; Lesser et al., 2013; Fig. 1.4). Given the high diversity within the genus Symbiodinium, the range of potential hosts and the range of environments (e.g., temperate to tropical ecosystems), it is expected that a spectrum of interactions,, from mutualism to parasitism, will likely be present across different host-symbiont partnerships (Sachs et al., 2011a; Sachs et al., 2011b; Lesser et al., 2013).

\subsection{Climate change and coral bleaching}

Symbiodinium cells are brown due to the presence of photosynthetic and accessory pigments in their chloroplasts. Healthy corals harbour millions of Symbiodinium cells per $\mathrm{cm}^{3}$ of tissue and therefore have the same brown colour. The loss of colour is most often due to symbiosis dysfunction and is referred to as coral

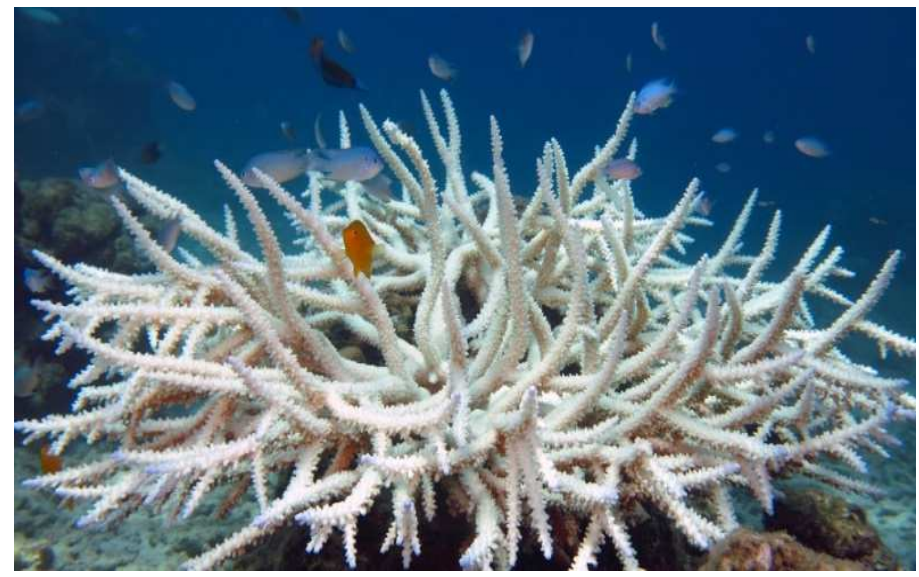

Fig. 1.5. Bleached coral, Acropora sp. Photo by Vardhan Pataker. 
bleaching (Weis, 2008; Fig. 1.5). Coral bleaching is a stress response to environmental change which involves the breakdown of the symbiosis and the loss of symbionts from host tissue and/or the loss of photosynthetic pigments within individual Symbiodinium cells (Weis et al., 2008). This process may be induced by a variety of physical and biological factors, including high and low seawater surface temperature (SST), UV irradiation, changes in salinity, bacterial infection, increased sedimentation and pollution (Jokiel and Coles, 1977; Coles and Brown, 2003; Hoegh-Guldberg and Fine, 2004). However, it is now well accepted that the synergetic effect of increased SST and irradiance, associated with global warming, is the major cause of mass bleaching (Hughes et al., 2003; Hoegh-Guldberg et al., 2007). An increase in SST of as little as $0.5-1.5^{\circ} \mathrm{C}$ for several weeks above the local maximum is sufficient to induce coral bleaching, since corals live close to their thermal threshold (Fitt et al., 2001).

There is evidence that both hosts and symbionts have the ability to tolerate stress by employing protective mechanisms such as increased heat shock proteins and antioxidant expression (Richier et al., 2003), as well as protective pigments (Salih et al., 2000; reviewed in Venn et al., 2008). Whether different survival mechanisms and tolerance will translate into different degrees of survival and adaptation to climate change still remains unclear and is the subject of active research (Lutz et al., 2015; Kenkel and Matz, 2016; Gajigan and Conaco, 2017).

\subsection{The adaptive bleaching theory}

Buddemeier and Fautin (1993) proposed the "adaptive bleaching hypothesis", which is based on the idea that bleaching is a strategy that promotes symbiont community change, which in turn can be beneficial to the coral host by allowing it to become repopulated with symbionts that are more suited to the new environment. Two different strategies for symbiont community change have been proposed: (1) Symbiont "shuffling". Corals can harbour one or more Symbiodinium types (Baker, 2003; Stat et al., 2006; Pochon and Gates, 2010; Fig 1.6A). This diversity of symbionts within individual corals theoretically creates the potential for quantitative change in the relative abundance of existing symbiont communities within colonies. There is some evidence for "shuffling" in the wild (e.g. Fitt, 2000; Mieog et al., 
2007; LaJeunesse et al., 2009; Kemp et al., 2014) and a low density of symbionts as a background community is required. For example, Kemp et al. (2014) showed that, under normal conditions, the stony coral Orbicella (=Montastraea) faveolata contained Symbiodinium A3, B17 and C7 as the dominant types, while type D1a was found at very low background densities. Recovery after the bleaching event of 2009 included a shift in the Symbiodinium populations and an increase in the densities of Symbiodinium types A3 and D1a, which are phylotypes known to have enhanced thermal tolerance. It has been shown that Symbiodinium populations eventually revert back to pre-bleaching genetic structure (LaJeunesse et al., 2009). (2) Symbiont "switching". Qualitative change by expulsion and acquisition of new symbionts acquired from the environment might be possible (Fig. 1.6B; Baker 2003), however this strategy has generated much controversy in the field (Baker, 2001a, 2003; Goulet, 2006), as there remains no support for it in the wild. Some corals are capable of acquiring symbionts from the water column, however this acquisition has been found to be temporary as these infections are not maintained as stable symbioses (Thornhill et al., 2006; Coffroth et al., 2010).

It has been suggested that bleaching might provide a window for opportunistic partnerships to become established (Rowan, 1998; Baker, 2002). For example, phylotypes of clade D Symbiodinium appear to become dominant in corals during and after bleaching, and exhibit a generalist lifestyle consistent with an opportunistic phenotype (LaJeunesse et al., 2009, 2010; Stat and Gates, 2011; Lesser et al., 2013; Kemp et al., 2014). Their dominance in bleached corals can result in an acquired thermal tolerance of $1.0-1.5^{\circ} \mathrm{C}$ (Berkelmans and van Oppen, 2006), which has short-term fitness benefits for the host (Donner et al., 2005, 2007; Donner, 2009). However, corals dominated by clade D Symbiodinium show significantly reduced rates of growth and reproduction, indicating lower long-term holobiont fitness (Jones and Berkelmans, 2010, 2011; Stat and Gates, 2011). This feature is consistent with a cheater strategy exhibited by many parasites (Herre et al., 1999; Sachs and Simms, 2006; Sachs et al., 2011a).

Repopulation by Symbiodinium following bleaching events is not very well understood (Kemp et al., 2014); in particular whether the specific host-symbiont combinations found in nature can change with time at a rate fast enough to match the pace of the changing conditions associated with climate change (Weis, 2008). 
A

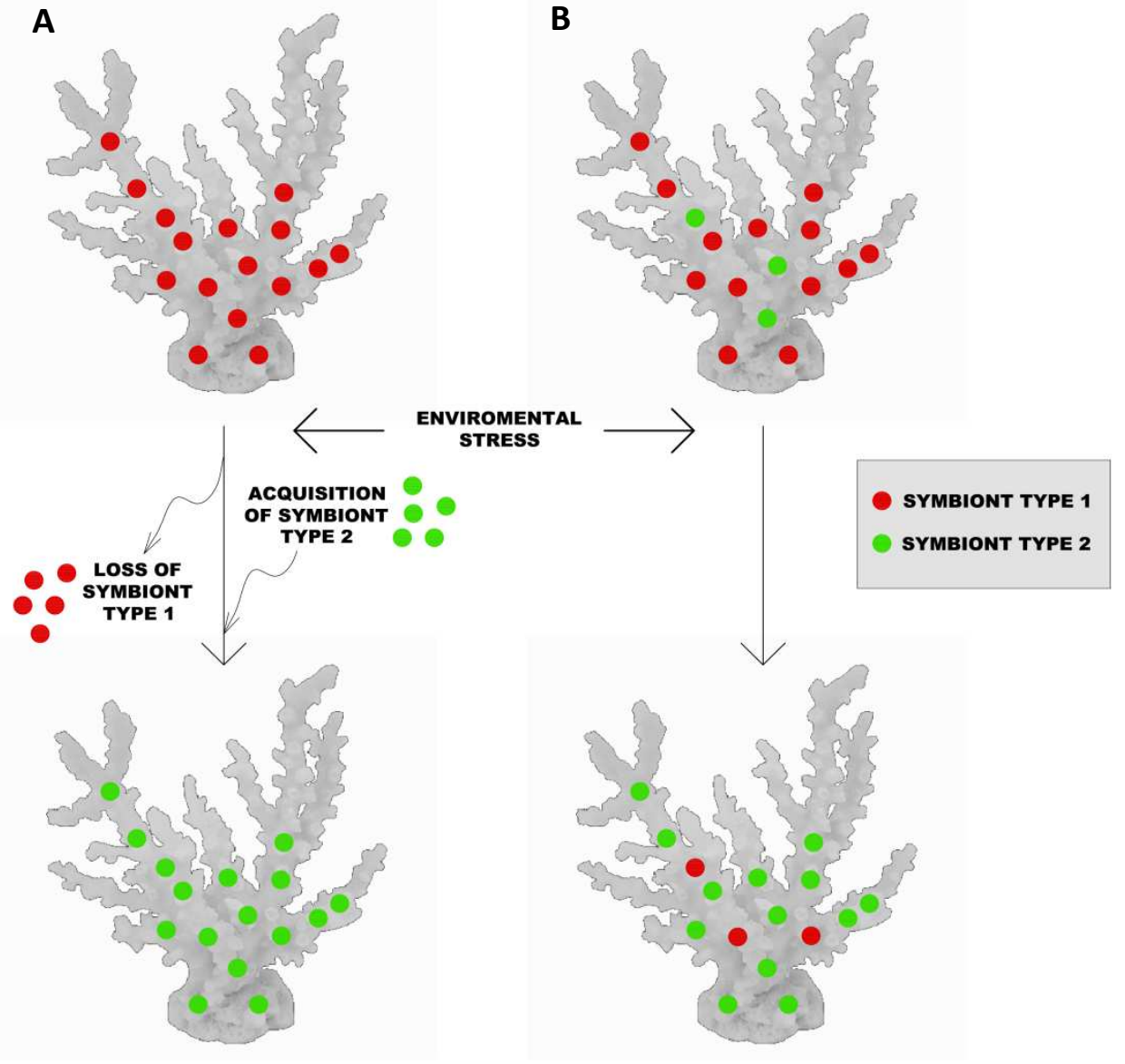

Fig. 1.6. Schematic diagram illustrating the two processes that can account for changes in the Symbiodinium population of a cnidarian host. (A) Expulsion and acquisition of new symbionts that are more suited for the new environmental conditions, described as "switching" and (B) a shift in the dominant algal type through competition between multiple types present in the host, referred as "shuffling" (redrawn from Stat et al., 2006).

\subsection{Aiptasia as a model system}

Much of the progress in molecular, cellular and developmental biology has come from studies using model organisms, such as E. coli, Drosophila and Arabidopsis. These organisms were chosen because they had characteristics that made them suitable to laboratory experiments, such as quick and easy growth of large numbers in the laboratory, the ability to produce clonal populations and the ability to subject them to biochemical and genetic manipulations (Weis et al., 2008). In recent years, the field of cnidarian-dinoflagellate 


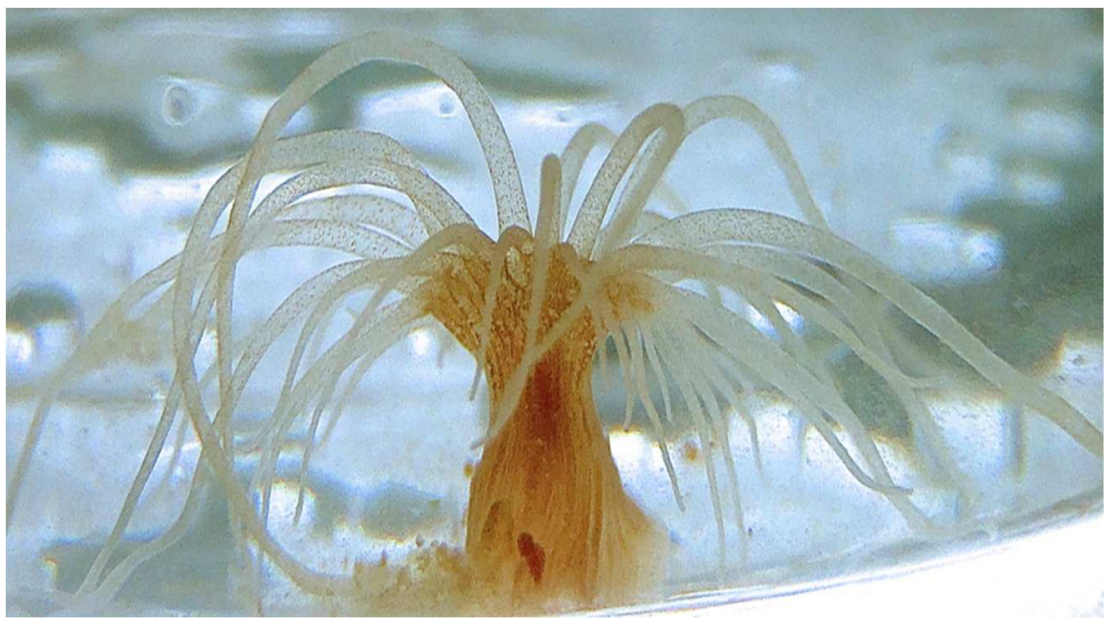

Fig. 1.7. Aiptasia sea anemone as a model system. In its symbiotic state. Photo by Clint Oakley.

symbiosis has moved toward the use of the sea anemone Exaiptasia pallida (Grajales and Rodríguez 2016; commonly referred to as 'Aiptasia') as a model system. Aiptasia is a tropical sea anemone, an anthozoan (like the major reef-building corals) that is also symbiotic with dinoflagellates of the genus Symbiodinium. The advantages of Aiptasia as a model compared to corals are its fast growth rate, ability to produce large clonal populations and ease of culture in the laboratory (Weis et al., 2008). Of particular note, and of huge advantage for the study of many aspects of the symbiosis, is that Aiptasia can be fully cleared of symbionts by temperature shock (Belda-Baillie et al., 2002) or by adding menthol (Matthews et al., 2015), maintained in the symbiont-free state for months or years (Kinzie et al., 2001) and re-infected at will with a variety of Symbiodinium types (Schoenberg and Trench, 1980a; Belda-Baillie et al., 2002; Weis et al., 2008; Starzak et al., 2014).

\subsection{Aim and specific objectives}

It is important to gain a deeper understanding of the potential for host cnidarians to form successful partnerships with different Symbiodinium types, and the mechanisms involved. This is particularly important for understanding the potential for adaptation of the symbiosis in the face of climate change, and global warming in particular. The aim of this research was to characterise the cellular and physiological events associated with hosting different symbiont types in a model cnidarian-dinoflagellate symbiosis, and how these events might contribute to observed patterns of host-symbiont specificity. To achieve this aim, I used a clonal culture of the model symbiotic sea anemone Aiptasia, which naturally contains $S$. minutum (ITS2 B1) only, as well as a range of cultured Symbiodinium genotypes possessing 
different physiologies (putatively ranging from less to more beneficial). The specific objectives were to:

1. Determine the effect of symbiont identity on the population dynamics of host colonisation (i.e. pattern and rate of symbiont proliferation) and holobiont physiology in the model sea anemone Aiptasia.

$\mathrm{H}_{0}$ : Different symbionts will present different outcomes for holobiont physiology, as well as exhibit different proliferation patterns, with the less beneficial taxa proliferating more rapidly and being more costly for the host.

2. Measure the competitiveness of a range of heterologous (i.e. non-native) symbionts in comparison to the homologous (i.e. native) symbiont, under both control and elevated temperatures in Aiptasia.

$\mathrm{H}_{0}$ : The homologous $S$. minutum will be dominant at the control temperature, but its competitiveness relative to more putatively opportunistic symbiont species will diminish at elevated temperatures.

3. Measure host apoptosis in response to homologous versus heterologous symbionts in Aiptasia, to assess how the capacity of the host to regulate its symbiont population is impacted by symbiont identity.

$\mathrm{H}_{0}$ : Anemones challenged with heterologous symbionts will show relatively high host cell apoptosis and therefore high caspase-3 activity, as a way of removing inappropriate symbionts, while anemones challenged with their homologous symbiont will exhibit lower apoptotic activity.

4. Measure symbiont cell-cycle progression of homologous versus heterologous symbionts in Aiptasia, to assess how the capacity of the host to regulate its symbiont population is impacted by symbiont identity.

$\mathrm{H}_{0}$ : Heterologous symbionts will show slower progression through the cell cycle than homologous symbionts, consistent with their differential rate of proliferation through the host's tissues. 


\title{
Chapter 2
}

\section{Symbiont identity influences patterns of symbiosis establishment, host growth and asexual reproduction in a model cnidarian-}

\author{
dinoflagellate symbiosis
}

\subsection{Introduction}

Among the most significant marine mutualisms are those between cnidarians and their photosynthetic dinoflagellate symbionts (Roth, 2014). These interactions, in particular between anthozoan cnidarians (e.g. corals and sea anemones) and dinoflagellates of the genus Symbiodinium, underpin the existence and success of coral reef ecosystems (Little et al., 2004; Wang et al., 2012; Lesser et al., 2013).

The genus Symbiodinium is very diverse, consisting of nine clades (A-I) and numerous subclades ('types') and species (LaJeunesse, 2002; Pochon and Gates, 2010) that display considerable physiological diversity (Schoenberg and Trench, 1980b; Chang et al., 1983; Fitt et al., 1981; Iglesias-Prieto et al., 2004; Robison and Warner, 2006; Hennige et al., 2009). Given this, different symbiont taxa can differentially affect host and hence 'holobiont' (i.e. the entire symbiotic entity) performance. For example, in corals, some members of clades A and $\mathrm{D}$ can be less beneficial to their hosts than members of clade $\mathrm{C}$, with lower rates of photosynthetic carbon fixation and translocation to the host, and reduced host fitness (Mieog et al., 2009; Leal et al., 2015). Alongside host phylotype and the prevailing environmental conditions, symbiont identity may therefore determine the fate of the symbiosis (Loram et al., 2007; Yuyama et al., 2016).

Such observations have led to the proposal that several Symbiodinium taxa might exhibit more opportunistic, and even parasitic traits than others (Stat and Gates, 2011; Lesser et al., 2013; Pettay et al., 2015; Silverstein et al., 2015). Starzak et al. (2014) tested this idea more 
broadly by modelling carbon fluxes in the model symbiotic anemone Aiptasia when colonised by a range of different Symbiodinium taxa. These models suggested that the usual, 'homologous' symbiont (Symbiodinium minutum, ITS2 type B1) forms a more beneficial symbiosis with Aiptasia than do several non-native, 'heterologous' symbiont types, such as A1.4, E and F5.2; Symbiodinium E and F5.2 were, on occasion, even observed to cause host mortality (Starzak et al., 2014). However, direct measures of host growth and reproductive output, to corroborate the models of Starzak and co-workers have not been performed. Indeed, we still know relatively little about the behaviour and physiology of homologous and heterologous Symbiodinium taxa in general during symbiosis establishment, including whether they exhibit similar spatial patterns of uptake and host colonisation. Such information is important for understanding the events that might occur during the establishment of novel host-symbiont pairings, as could potentially occur after a coral bleaching event. Furthermore, such details are important for understanding the drivers of host-symbiont specificity; that is, the reasons why many hosts are found in association with only one or a few Symbiodinium taxa, and vice versa (Baker, 2003; LaJeunesse et al., 2004; Coffroth et al., 2010).

This study addressed these knowledge gaps using the sea anemone Exaiptasia pallida (Grajales and Rodríguez, 2014; commonly referred to as 'Aiptasia'), a widely-adopted model system for the study of reef-building corals and the cnidarian-dinoflagellate symbiosis (Weis et al., 2008; Baumgarten et al., 2015). The current study measured the influence of symbiont diversity on host colonisation dynamics, as well as host performance. I employed five different Symbiodinium species or types, representing a wide range of clades (A-E) and physiologies, and including the free-living and relatively heterotrophic Symbiodinium voratum (type E; Jeong et al., 2012) and the thermally-tolerant but opportunistic Symbiodinium trenchii (type D1a; Pettay and Lajeunesse 2009). Specifically, I asked whether symbiont identity affects: (1) symbiont cell proliferation; (2) colonisation pattern inside the host; (3) photosynthetic performance; and (4) host fitness. In doing so, I not only provide further insight into the cellular events that occur during host colonisation by symbiotic dinoflagellates and the physiological implications of symbiont diversity, but also a baseline from which to conduct more detailed studies of symbiosis establishment and function with the model Aiptasia system. 


\subsection{Material and Methods}

\subsubsection{Experimental organisms}

A clonal culture of the symbiotic sea anemone Aiptasia (culture ID: NZ1), originally from the Indo-Pacific region, was grown in the lab at $25{ }^{\circ} \mathrm{C}$ and an irradiance of $100 \mu \mathrm{mol}$ photons $\bullet$ $\mathrm{m}^{-2} \cdot \mathrm{s}^{-1}$ (12-h light/12-h dark), and fed twice weekly with freshly hatched Artemia nauplii. To generate aposymbiotic (i.e. symbiont-free) anemones, animals were incubated in a solution of $0.27 \mathrm{mM}$ menthol/FSW $(0.22 \mu \mathrm{m}$-filtered seawater $)$ for $8 \mathrm{~h}$, after which the menthol/FSW was removed and the anemones incubated in $5 \mu \mathrm{M}$ DCMU/FSW (3-(3,4dichlorophenyl)-1,1-dimethylurea) overnight as described by Matthews et al., (2015), with the treatment being repeated daily for four weeks or until no symbionts were present, as determined by confocal microscopy (IX81, Olympus New Zealand; $635 \mathrm{~nm}$ laser, 655-755 $\mathrm{nm}$ emission filter).

Cultured Symbiodinium from five clades were used as inoculates (Table 2.1). Species names of these dinoflagellates are used where available; otherwise ITS2 type is given. The algae were sub-cultured from long-term (> 5 years) laboratory stocks and grown in silica-free $\mathrm{f} / 2$ medium (Sigma-Aldrich, Auckland, New Zealand) at $25^{\circ} \mathrm{C}$ and an irradiance of $100 \mu \mathrm{mol}$ photons $\cdot \mathrm{m}^{-2} \cdot \mathrm{s}^{-1}$, on a 12-h light/12-h dark regime. All cultures were sampled for experimental use during the log phase of growth

Table 2.1. Identity and geographical origin of Symbiodinium cultures and the original host species if known

\begin{tabular}{|c|c|c|c|c|c|}
\hline Culture ID & $\begin{array}{l}\text { Original host } \\
\text { source }\end{array}$ & $\begin{array}{c}\text { Geographical } \\
\text { location }\end{array}$ & $\begin{array}{c}\text { Symbiodinium } \\
\text { species }\end{array}$ & Subclade & $\begin{array}{c}\text { Relationship } \\
\text { to the host }\end{array}$ \\
\hline FlAp2 & Exaiptasia pallida & Long Key, Florida & S. minutum & B1 & Homologous \\
\hline CCMP2467 & Stylophora pistillata & Gulf of Aqaba & S. microadriaticum & A1 & Heterologous \\
\hline $\mathrm{Mp}$ & Mastigias papua & Palau & Symbiodinium sp. & $\mathrm{C} 3$ & Heterologous \\
\hline Ap2 & Unknown anemone sp. & Okinawa & S. trenchii & D1a & Heterologous \\
\hline CCMP421 & $\begin{array}{l}\text { Free-living (host, if } \\
\text { any, unknown) }\end{array}$ & $\begin{array}{l}\text { Wellington Harbour, } \\
\text { NZ }\end{array}$ & S. voratum & E & Heterologous \\
\hline
\end{tabular}




\subsubsection{Inoculation of aposymbiotic anemones with symbionts}

Following one week of starvation, a total of 312 aposymbiotic anemones $(n=60$ anemones for each Symbiodinium type/species; $n=12$ uninoculated anemones as a negative control) of similar size (2-3 $\mathrm{mm}$ oral disc diameter) were transferred to $400 \mathrm{ml}$ clear plastic jars (one anemone per jar) filled with FSW, and allowed to settle for three days. Each algal culture was diluted in $25 \mathrm{ml} \mathrm{FSW}$ and one drop of Artemia nauplii suspension added, to give a final concentration of $\sim 1 \times 10^{6}$ cells $/ \mathrm{ml}$. The anemones were inoculated with one of the five different symbiont cultures, by pipetting $1 \mathrm{ml}$ of this dinoflagellate suspension onto the oral disc of each anemone with a glass pipette. Colonisation was then monitored over a period of eight weeks, with sampling at 1, 2, 4, 6 and 8 weeks post-inoculation. During this time, the anemones were fed twice weekly with Artemia nauplii, with each feeding followed by a water-change. Seawater temperature was maintained at $25{ }^{\circ} \mathrm{C}$ in a controlled temperature room, and irradiance was held at $\sim 100 \mu \mathrm{mol}$ photons $\bullet \mathrm{m}^{-2} \cdot \mathrm{s}^{-1}$ on a 12-h light/12-h dark cycle.

\subsubsection{Symbiont proliferation}

\subsubsection{Spatial pattern of colonisation}

To determine the effect of symbiont identity on the spatial pattern of host colonisation, ten randomly selected anemones from each treatment were chosen at each sampling point, and assessed via chlorophyll autofluorescence measured with a confocal microscope (details above). A drop of relaxation solution (50\% $0.37 \mathrm{M} \mathrm{MgCl}_{2}$ in $\mathrm{dH}_{2} \mathrm{O}$ and $50 \% \mathrm{FSW}$ ) containing $1.5 \%$ of agar was added to a subset of the sampled anemones on a fluorodish (World Precision Instruments) ( $n=5$ per treatment), and z-stack images generated (section thickness $<10 \mu \mathrm{m})$. The multiple z-stack sections were then summed into a single photo, regions of interest marked, and fluorescence measured with ImageJ software (V. 1.48, National Institute of Health, USA). The following equation was used to calculate 
fluorescence intensity, as a proxy for algal number:

Correct Fluorescence $=$ IntDen $_{S}-\left(\right.$ Area $_{S}-$ Mean $\left._{B}\right)$

Where IntDen = fluorescence measured using ImageJ $\mathrm{S}=$ sample; $\mathrm{B}=$ background.

\subsubsection{Quantification of symbionts during colonisation}

Following confocal microscopy, each anemone was homogenized separately in $500 \mu$ FSW (IKA T-10 tissue lyser, ThermoFisher Scientific) and the homogenate centrifuged (Sigma 3$16 \mathrm{k}$ ) for $5 \mathrm{~min}$ at $400 \times \mathrm{g}$, to separate algal cells from anemone tissue. A 100- $\mu \mathrm{l}$ aliquot was removed from the supernatant (host fraction) for protein determination and the remaining supernatant discarded. The pellet containing the dinoflagellate cells was re-suspended in 200 $\mu \mathrm{FSW}$. A $50-\mu \mathrm{l}$ aliquot was added to $50 \mu \mathrm{l}$ dimethyl sulfoxide buffer for DNA sequencing (see below), and the remaining $150 \mu \mathrm{l}$ were used for algal cell counts. The samples were stored at $-20{ }^{\circ} \mathrm{C}$ until further analysis. Host protein content was determined via the Bradford assay (Bradford, 1976) and cell counting was performed with a haemocytometer (Improved Neubauer) with six replicate counts per sample. Cell density (symbionts per mg protein) was then calculated.

\subsubsection{Photo-physiology and host performance}

\subsubsection{Photosynthesis and respiration of the host-symbiont partnership}

Maximum gross photosynthetic and dark respiratory $\mathrm{O}_{2}$ fluxes were measured for all hostsymbiont partnerships at the end of the 8 -week experiment ( $n=4$ for each treatment). Individual anemones were placed at $25^{\circ} \mathrm{C}$ in a $10 \mathrm{ml}$ glass chamber, fitted with a magnetic stir bar protected by nylon mesh, filled with FSW and sealed by a glass lid with rubber Oring, into which an oxygen electrode (FIBOX 3-fibre-optic oxygen metre, PreSens GmbH) was inserted. Animals were allowed to settle for $30 \mathrm{~min}$ prior to the beginning of the experiment, with the chamber remaining unsealed and hence open to the air during this time. The respiration rate $\left(\mathrm{ml} \mathrm{O}_{2} \bullet \mathrm{h}^{-1}\right)$ was measured in darkness for an hour, followed by $1 \mathrm{~h}$ of 
illumination by a Thorn PAR lamp $38150 \mathrm{~W}$ at $400 \mu \mathrm{mol}$ photons $\bullet \mathrm{m}^{-2} \cdot \mathrm{s}^{-1}$. Each anemone was then homogenized in $500 \mu \mathrm{l} \mathrm{FSW}$ and host protein content determined as described before. The rates of gross photosynthesis and respiration were normalized to host protein, and the ratio of hourly gross photosynthesis to respiration $(\mathrm{P}: \mathrm{R})$ calculated as a proxy for autotrophic potential of the symbiosis.

\subsubsection{Host growth and asexual reproduction}

Host growth between the start and end of the experiment (i.e. eight weeks after inoculation) was estimated via the change in oral disc diameter, assuming a starting diameter of 2-3 mm ( $n=10$ for each treatment). At the final time-point, anemones were incubated in their containers in $\mathrm{MgCl}_{2}$ relaxation solution (see above) until they were not responsive to disturbance, and then the containers placed onto millimetric graph paper for measurement of the oral disc diameter. Asexual reproduction was measured as the number of pedal lacerates generated by each anemone between the start of the experiment and the sampling point, throughout the duration of the experiment ( $n=10$ for each treatment and time-point).

\subsubsection{DNA sequencing}

To confirm that symbiont populations in animals matched the populations used for inoculation, symbiont identity was verified for each culture and a subset of inoculated anemone samples ( $n=3$ samples per culture or sample). Symbiodinium samples containing DMSO (see above) were re-suspended, and $10 \mu \mathrm{l}$ of each sample added to a new tube containing $50 \mathrm{mg}$ glass beads (G1152-10G, Sigma-Aldrich) and placed in a TissueLyser (Qiagen, Bio-Strategy) bead beater for $1 \mathrm{~min}$ at $50 \mathrm{~Hz}$. Ninety microliters of $\mathrm{ddH}_{2} \mathrm{O}$ were added, and lysed cell material was pelleted at $16,100 \times \mathrm{g}$ for $10 \mathrm{~min}$ at $4{ }^{\circ} \mathrm{C}$. A $50 \mu \mathrm{l}$-aliquot of the resulting supernatant was transferred to a new tube containing $50 \mu$ l cold moleculargrade isopropanol, and samples were re-pelleted as described above. Samples were washed twice in $200 \mu \mathrm{l} 70 \%$ ethanol, centrifuged and then dried at room temperature. Fifty microliters of $1 \mathrm{M}$ Tris ( $\mathrm{pH}$ 8) were added to each tube and samples were further extracted by bead beating at $30 \mathrm{~Hz}$ for $1 \mathrm{~min}$ and then stored at $-20{ }^{\circ} \mathrm{C}$ until further analysis. Polymerase chain reaction (PCR) was performed using the following Symbiodinium-specific ITS2 
(internal transcribed spacer region 2) primers: forward primer (ITS2infor) 5'GAATTGCAGA ACTCCGTG-3'; reverse primer (ITS2CLAMP) 5'-CGCCCGCCGC GCCCCGCGCC CGTCCCGCCG CCCCCGCCC GGGATCCATA TGCTTAAGTT

CAGCGGGT-3'. Reactions were performed using the MyTaq ${ }^{\mathrm{TM}}$ Mix in a total volume of 25 $\mu \mathrm{l}$ with an amplification profile consisting of: 1 cycle of $3 \min 95^{\circ} \mathrm{C} ; 40$ cycles of $15 \mathrm{~s} 95^{\circ} \mathrm{C}$, $15 \mathrm{~s} 56{ }^{\circ} \mathrm{C}$ and $10 \mathrm{~s} 72{ }^{\circ} \mathrm{C}$; and a final hold temperature of $4{ }^{\circ} \mathrm{C}$. PCR products were sequenced by Macrogen Inc. (Seoul, South Korea). Sequences were aligned using Geneious Pro 4.8.5 and a BLAST search was performed in the National Centre for Biotechnology Information (NCBI) database to identify the sequences. In all cases, the expected genotypes were present both at the beginning and end of the experiment. Multiple genotypes were never detected in any sample.

\subsubsection{Statistical analysis}

Analysis of Variance (ANOVA) followed by either Tukey or Bonferroni post hoc tests was performed on the data using STATISTICA 10 and SPSS, except in the following cases. Log transformation was conducted on symbiont density data to achieve a normal distribution followed by Welch's ANOVA, since constant variance could not be assumed. Welch's ANOVA was also performed on dark respiration data for the same reason. This test was followed by Games-Howell post hoc analysis. A chi-square test was conducted on asexual reproduction data to test the effect of time*algal identity. Results are expressed as mean \pm standard error (SE).

\subsection{Results}

All Symbiodinium taxa initially colonised Aiptasia. There was no cross-contamination of symbionts between treatments (see Appendix 2) and aposymbiotic anemones remained symbiont-free throughout the experiment. 


\subsubsection{Symbiont proliferation in hosts}

Symbiont cell density (algal cells $\cdot \mathrm{mg}$ host protein $^{-1}$ ) was significantly different between anemones inoculated with different Symbiodinium taxa (Fig. 2.1; Welch's ANOVA, F $F_{19,58}=$ 130.087, $P<0.0001)$. The homologous $S$. minutum was the most successful at colonising hosts, reaching its peak density two weeks after inoculation and achieving a maximum density that was 13 -fold higher than that of S. trenchii, the second most successful symbiont, at that same time-point (Games-Howell post hoc, $P<0.0001$ ). Between Weeks 2 and 8, the density of $S$. minutum decreased by $62 \%$, to $3.35 \times 10^{6} \pm 3.18 \times 10^{5}$ cells $\cdot \mathrm{mg}$ protein $^{-1}$, which was still significantly higher than that of all other symbionts (Games-Howell post hoc, $P<0.006)$. S. microadriaticum and $S$. trenchii populations exhibited a slower initial increase in density than $S$. minutum, but by Week 4 their densities peaked at similar levels to those of S. minutum (Games-Howell post hoc, $P>0.05$ for both comparisons; Fig. 2.1);

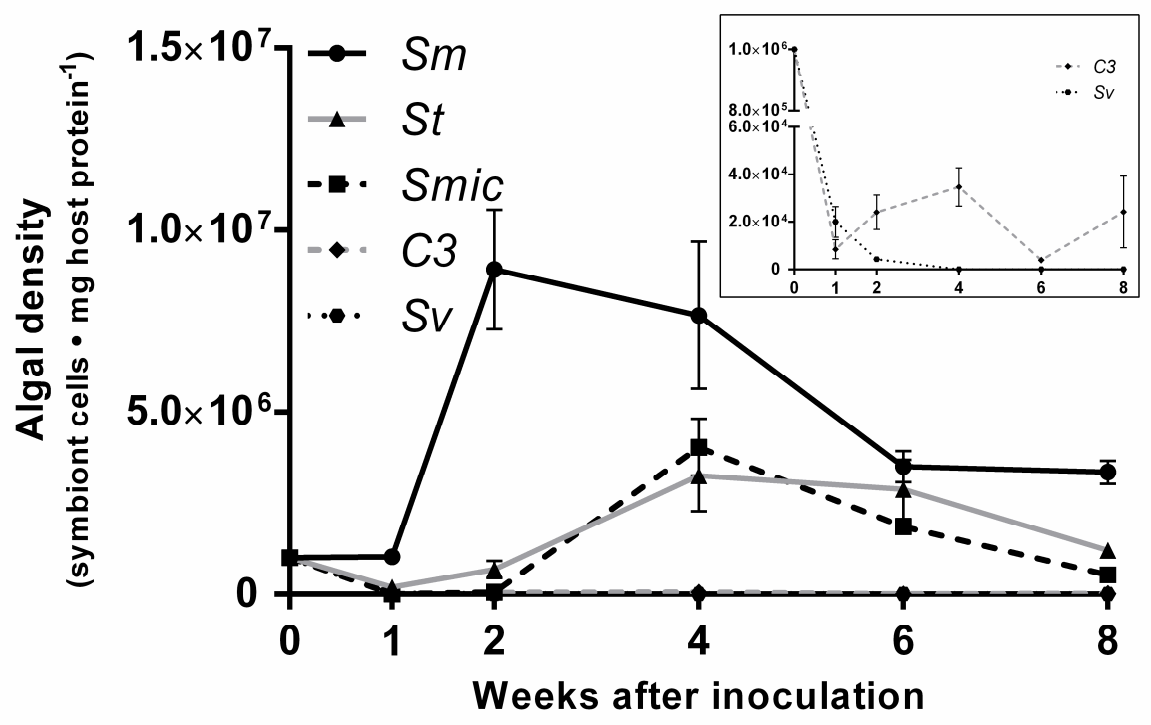

Fig. 2.1. Colonisation success in host Aiptasia varied with symbiont identity. Symbiont density (symbiont cells $\bullet$ mg host protein $^{-1}$ ) in host anemones was quantified for eight weeks after inoculation $(\mathrm{n}=10$ anemones per treatment per time point). Sm $=$ Symbiodinium minutum; $S t=S$. trenchii; Smic $=S$. microadriaticum; C3 = Symbiodinium phylotype C3; Sv $=S$. voratum. Points are means \pm SE. 
their densities then declined during the remainder of the experiment, by $71 \%$ and $58 \%$, respectively, so that they were again significantly lower than the density of S. minutum (Games-Howell post hoc, $P<0.001$ ). Symbiodinium C3 and $S$. voratum were the least successful at colonisation. The population density of C3 was significantly less than that of $S$. minutum and S. trenchii (Games-Howell post hoc, $P<0.05$ ) throughout the experiment, and significantly less than $S$. microadriaticum from Week 4 onwards. In comparison, S. voratum initially colonised the host, but after two weeks its density declined dramatically, such that by Week 4 and thereafter, no symbionts were observed (Fig. 2.1).

The different Symbiodinium taxa showed similar spatial patterns of colonisation through host tissues, albeit at different temporal rates (Fig. 2.2A-C). Colonisation in the tentacles did not progress smoothly from the tentacle base to tip; rather, it was patchier, with punctuated bursts of algal growth in spatially disparate portions of the tentacle (Fig 2.2A\&B). Furthermore, the timing of these various spatial events differed substantially between the different Symbiodinium (Fig. 2.2C), consistent with the 'strength' of overall colonisation as shown in Figure 2.1. Of particular note, the relatively unsuccessful Symbiodinium C3 only ever colonised the oral disc and tentacles, and by Week 8 appeared in the oral disc alone, suggesting that colonisation was failing. 

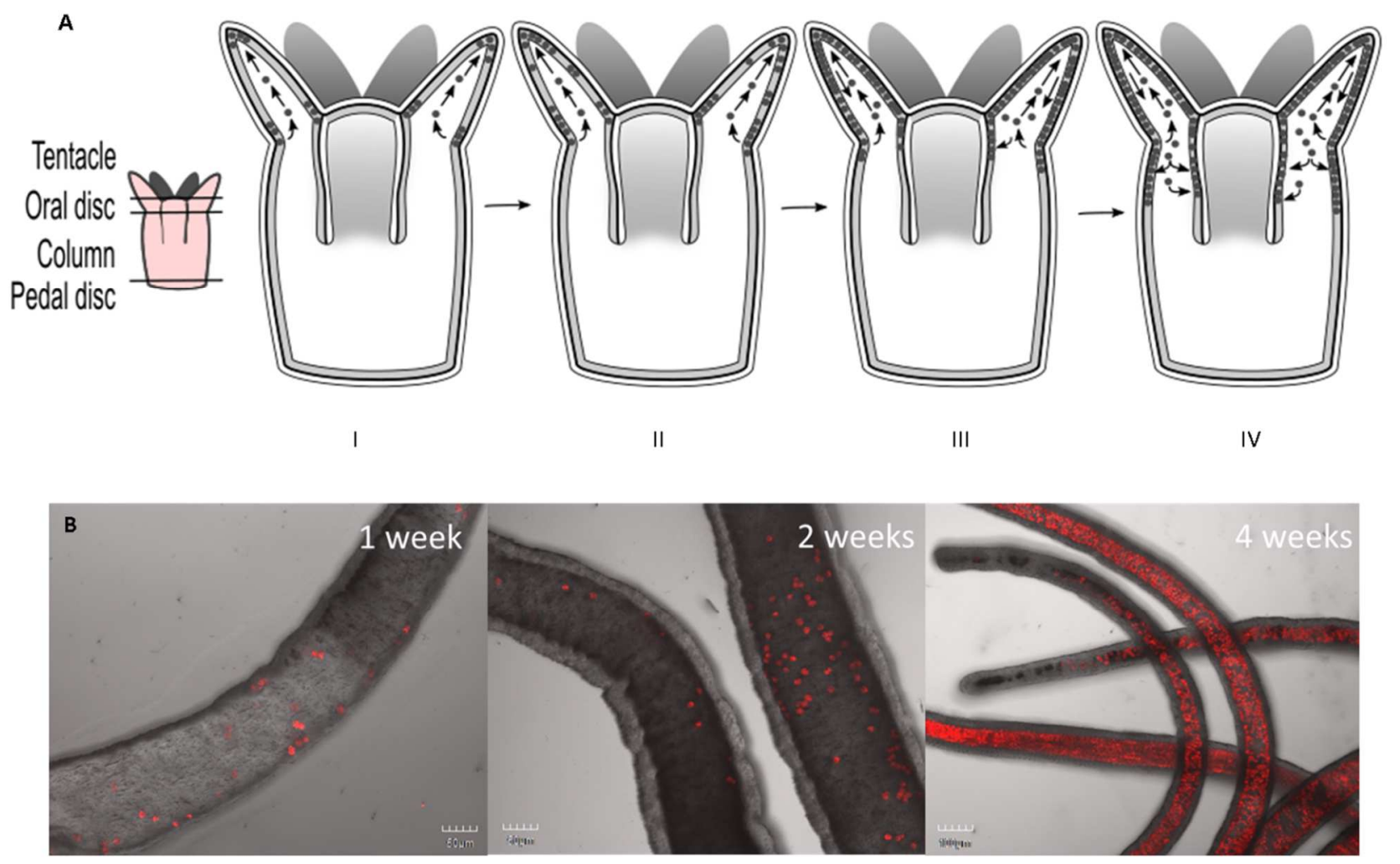

c

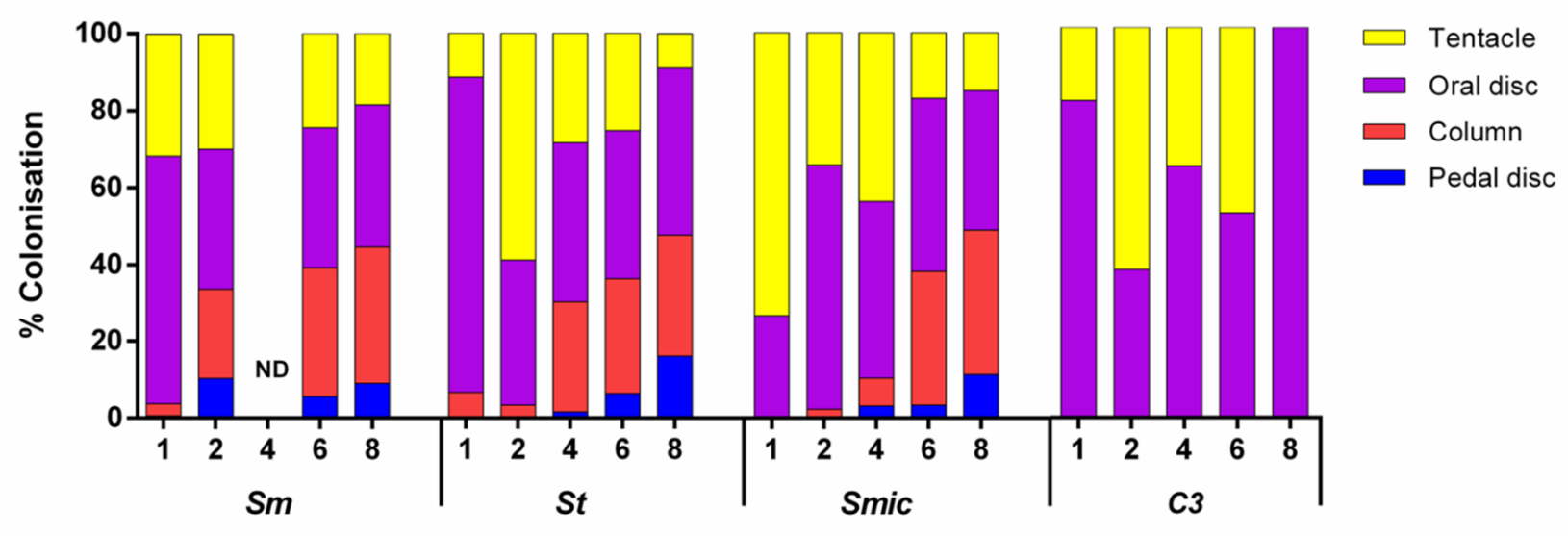

Weeks after inoculation

\section{Symbiont species}

Fig. 2.2. Spatial pattern of symbiont colonisation in Aiptasia is similar for different symbionts. Spatial pattern and colonisation across four body sections in the anemone host tentacles, oral disc, column and pedal disc (see panel A) - quantified for eight weeks after inoculation ( $n=2-6$ anemones per treatment and time-point). (A) Schematic representation: I. symbionts taken up by the anemone and concentrated in the oral disc. Proliferation continues to the tentacles and exhibits a patchy pattern, suggesting symbiont migration via the 
gastrovascular cavity; II. Colonisation of the tentacles continues through mitotic division and gastrovascular migration; III. Tentacles are fully colonised and the symbiont cells begin to colonise the upper part of the column; IV. The symbionts continue to colonise the column from top to bottom, ultimately reaching the pedal disc. (B) Confocal images of tentacle colonisation by Symbiodinium trenchii over four weeks. S. trenchii rather than S. minutum is shown here, since $S$. trenchii was slower to colonise the host and hence the spatial patterns are clearer across time; (C) Spatial pattern of colonisation by different symbionts, presented as the percentage of total symbiont chlorophyll fluorescence occurring in the four different body regions of the anemone over time. $S m=$ Symbiodinium minutum; $S t=S$. trenchii; Smic $=S$. microadriaticum $; \mathrm{C} 3=$ Symbiodinium phylotype C3; Sv $=$ S. voratum .

\subsubsection{Photo-physiology and hosts performance}

\subsubsection{Photosynthesis and respiration of the host-symbiont partnership}

Gross photosynthesis $\left(\mathrm{mg} \mathrm{O}_{2} \bullet \mathrm{mg}\right.$ protein $\left.^{-1} \bullet \mathrm{h}^{-1}\right)$ was significantly different when Aiptasia formed a symbiosis with the different Symbiodinium species or types (one-way ANOVA, $\mathrm{F}_{4}$, $13=12.214, P<0.001$; Fig. $2.3 \mathrm{~A}$ ). At the end of the experiment, anemones containing $S$. minutum or $S$. trenchii photosynthesized at a similar rate, as did those anemones containing $S$. microadriaticum or type $\mathrm{C} 3$, however the photosynthetic rate with the former two symbionts was faster than that with the latter two (Tukey post hoc, $P<0.01$ for all comparisons). In contrast, dark respiration rate $\left(\mathrm{mg} \mathrm{O}_{2} \cdot \mathrm{mg}\right.$ protein $\left.^{-1} \bullet \mathrm{h}^{-1}\right)$ was not affected by symbiont identity (Fig. 2.3A; Welch's ANOVA, $F_{4}, 5.5=3.043, P>0.05$ ). As a result, there was a significant difference in the P:R ratio between anemones hosting the different symbionts at the end of the experiment (one-way ANOVA, $\mathrm{F}_{3}, 10=11.420, P<0.05$ ), with $S$. minutumcolonised anemones having a significantly higher ratio than those containing $S$. microadriaticum or C3 (Tukey post hoc, $P<0.05$ for both comparisons), but not $S$. trenchii (Fig. 2.3B). 


\subsubsection{Host growth and asexual reproduction}

Host growth, estimated at the end of the experiment from the oral disc diameter, was significantly impacted by Symbiodinium identity (one-way ANOVA, F5, $44=19.820, P<$ 0.0001). In particular, anemones colonised by $S$. minutum were significantly larger at Week 8 than anemones colonised by any of the heterologous symbionts (Tukey post hoc, $P<0.0001$ for all comparisons; Fig. 2.4A), while anemones colonised by heterologous symbionts were no larger than aposymbiotic anemones (Tukey post hoc, $P>0.9$ for all comparisons). Anemones containing S. minutum grew by an average of $9.22 \pm 0.68 \%$ between the beginning and end of the experiment, while anemones containing S. trenchii and S. microadriaticum grew by just $1.9 \pm 1.06 \%$ and $1.54 \pm 1.05 \%$, respectively. Anemones containing C3 and $S$. voratum showed, on average, negative growth of $(-1.91) \pm 0.65 \%$ and $(-1.71) \pm 1.08 \%$, respectively. By comparison, aposymbiotic anemones grew $0.28 \pm 0.88 \%$.

A

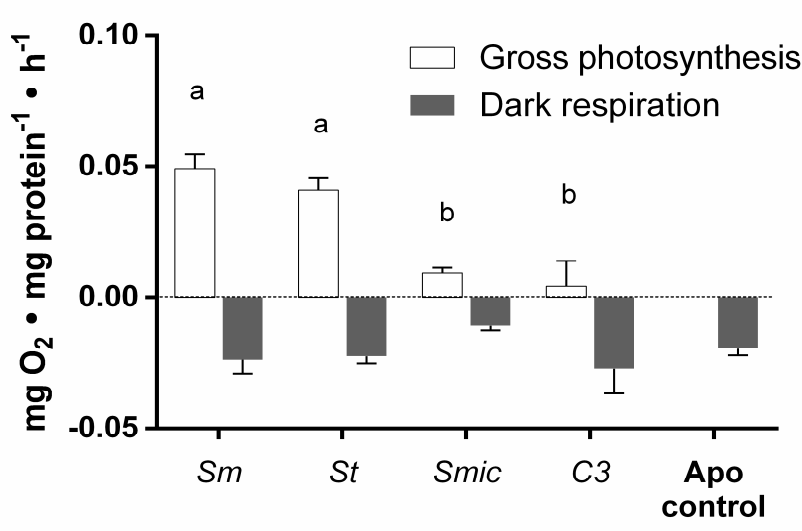

B

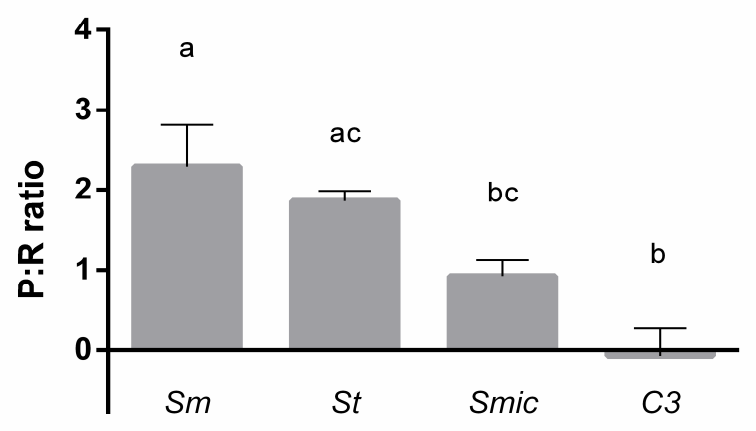

Symbiodinium species
Fig. 2.3. Gross photosynthesis and dark respiration of the holobiont varied with symbiont identity. Photo-physiological performance $(\mathrm{mg}$ $\mathrm{O}_{2} \cdot \mathrm{mg} \operatorname{protein}^{-1} \bullet \mathrm{h}^{-1}$ ) of the holobiont was quantified eight weeks after inoculation. (A) Gross photosynthesis (white bars) and total symbiosis dark respiration (grey bars); (B) P:R ratio. $S m=$ Symbiodinium minutum; $S t=S$. trenchii; Smic $=$ S. microadriaticum; C3 = Symbiodinium phylotype C3; Sv $=S$. voratum. Bars represent means \pm $\mathrm{SE}, \mathrm{n}=3-4$ anemones per treatment. Different letters above bars indicate significant differences between treatments $(p<0.05)$. 


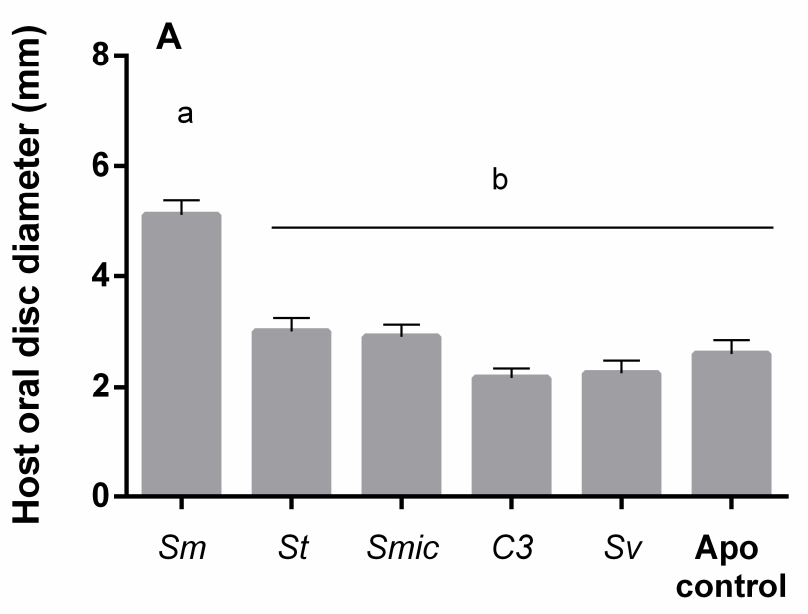

Symbiodinium species

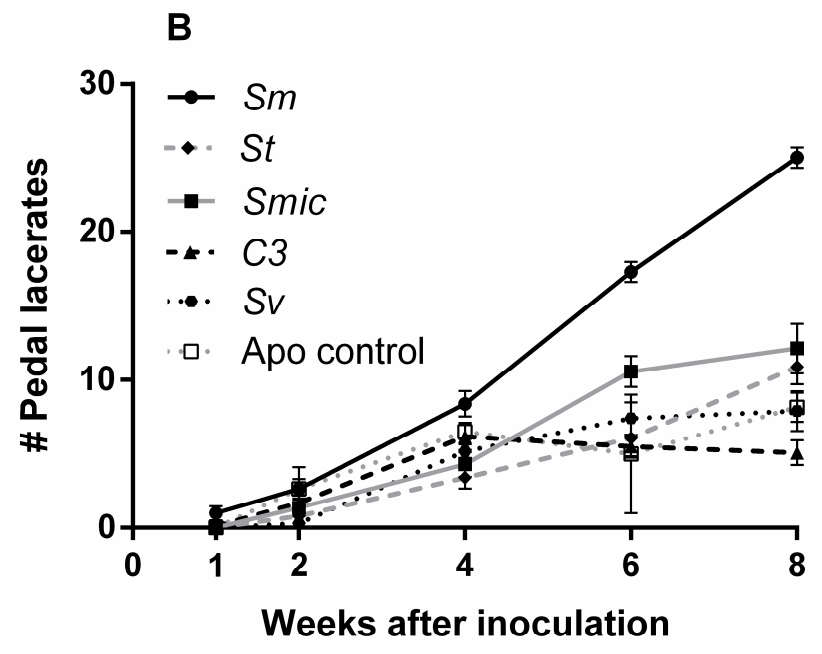

Fig. 2.4. Host performance varied with symbiont identity. (A) Host growth estimated from the change in oral disc diameter $(\mathrm{mm})$, quantified eight weeks after colonisation $(\mathrm{n}=10$ for each treatment); (B) asexual reproduction via pedal laceration measured by count up pedal lacerates quantified over eight weeks after inoculation ( $\mathrm{n}=10$ anemones per treatment and time-point). $S m=$ Symbiodinium minutum; $S t=S$. trenchii $;$ Smic $=S$. microadriaticum; $\mathrm{C} 3=$ Symbiodinium phylotype C3; Sv = S. voratum. Values are mean $\pm \mathrm{SE}$.

Consistent with the differences in size and growth, after eight weeks anemones colonised by S. minutum had produced significantly more pedal lacerates than did anemones colonised by the other symbionts (Chi square, Bonferroni post hoc, $P<0.0001$ for all comparisons; Fig. 2.4B). S. minutum-colonised anemones produced an average of $25 \pm 0.58$ pedal lacerates per anemone between the start of the experiment and Week 8, compared to $12 \pm 1.48,11 \pm 1.20$, $7 \pm 1.11$ and $5 \pm 0.85$ pedal lacerates when in symbiosis with $S$. microadriaticum, S. trenchii, S. voratum and type C3, respectively. By Week 8, S. microadriaticum-colonised anemones produced a similar number of pedal lacerates to $S$. trenchii-colonised anemones, and both produced a higher number of pedal lacerates than anemones inoculated with $S$. voratum or type C3 (Chi square, Bonferroni post hoc, $P<0.05$ ). Aposymbiotic anemones produced $8 \pm$ 0.76 pedal lacerates per anemone by Week 8 , a similar number to those produced by 
anemones inoculated with $S$. microadriaticum, S. trenchii or $S$. voratum (Chi square, Bonferroni post hoc, $P>0.05$ for all comparisons), but a significantly higher number than for anemones inoculated with C3 (Chi square, Bonferroni post hoc, $P<0.05$ ).

\subsection{Discussion}

\subsubsection{Colonisation success varies with symbiont identity}

Colonisation success varied with symbiont identity, with the homologous $S$. minutum being fastest to proliferate and invade host tissues. Consistent with my results, previous colonisation studies have shown that symbiotic algae are more immediately successful when originally isolated from the same host species (Kinzie and Chee, 1979; Schoenberg and Trench, 1980a; Davy et al., 1997b; Belda-Baillie et al., 2002; Harii et al., 2009; Starzak et al., 2014). For example, Weis et al. (2001) showed that homologous symbionts were able to colonise nearly all Fungia scutaria larvae inoculated, and reach higher densities inside each larva than heterologous symbionts; while some of these heterologous symbionts could not colonise the larvae at all.

In contrast, some of the other symbionts in my study failed or struggled to form a symbiosis with Aiptasia. In particular, C3 was taken up by the host but there was no evidence of symbiont proliferation, while $S$. voratum did not persist inside the host beyond four weeks. Previous studies have likewise shown differential success of heterologous symbionts in Aiptasia. For example, Belda-Baillie et al. (2002) reported that adult Aiptasia could form only a limited symbiosis with cultured Symbiodinium A and was unable to form a symbiosis with a C-type and a free-living A-type, even after three months. Furthermore, S. voratum has previously been reported to be incapable of forming a symbiosis with both larval and adult Aiptasia, a finding that is consistent with my observations here (Xiang et al., 2013; Wolfowicz et al., 2016). My results are, however, somewhat different from those of Starzak et al. (2014), who reported that S. voratum (culture ID CCMP421) was, on occasion, able to proliferate rapidly and reach high densities inside Aiptasia. The reason for the difference between this and the current study is unclear, not least given that the same cultures of Symbiodinium (CCMP421) and Aiptasia (NZ1) were used in both studies, though it is 
important to note that Starzak and co-workers also reported a relatively low percentage of colonisation success and a high incidence of host mortality when anemones were inoculated with $S$. voratum.

The failure of Symbiodinium C3 and S. voratum to proliferate and persist in Aiptasia, respectively, despite uptake from the external environment, highlights the role of postphagocytotic events in the establishment of a successful symbiosis, including the initiation of a functional molecular cross-talk between the host and symbiont. Exactly what this molecular cross-talk involves remains unclear, though it is likely that molecular signals and/or nutritional interplay have a role in the evasion of host immunity by the invading symbiont, and the prevention of symbiont loss via the likes of host expulsion or host-cell apoptosis (reviewed by Davy et al., 2012). The failure of $S$. voratum to persist is not surprising given its free-living origin, as well as the fact that this species is relatively large compared to other symbiont species. I did not measure cell size here, however a recent paper by Biquand et al. (2017) suggests that the colonisation success of Symbiodinium in Aiptasia is negatively correlated with symbiont cell size. My results, where different symbiont species or types had very different fates in symbiosis with Aiptasia, provide a baseline for further study of the molecular signals and events that dictate symbiosis success in this important model system.

While there were marked differences between the colonisation success of the different symbionts, the spatial patterns of colonisation were very similar, with algae first appearing in the oral disc, before spreading to the tentacles, then the column and finally the pedal disc. This pattern is perhaps not surprising given that the oral disc is likely the first point of contact between host and symbiont. The patchy pattern of symbiont proliferation in host tentacles (Fig. 2.2A) suggests that algal clusters arise from mitotic division of a single invading cell, with the algal symbionts moving either through the anemone's tissue or via transport in the gastrovascular cavity. Symbionts have been shown to pass between host cellular compartments during host oogenesis or in larvae in a range of cnidarians (Benayahu et al., 1992; Benayahu and Schleyer, 1998; Hirose et al., 2000, 2001; Davy and Turner, 2003; Marlow and Martindale, 2007), however the putative migration distances to generate the patterns seen in my study, even in small adult Aiptasia, would be much greater and hence this mechanism of proliferation seems implausible here. It is more likely that the symbionts exit host cells, move through the gastrovascular cavity via ciliary water currents and invade other 
host cells elsewhere in the animal. While I could not confirm the presence of symbiont cells in the gastrovascular cavity of the tentacles due to the technical limitations of my confocal method, Parrin et al. $(2012 ; 2016)$ observed within-colony symbiont migration in soft corals as a response to thermal stress, providing support for this proposed mechanism. This topic warrants detailed investigation.

\subsubsection{Host photo-physiology and performance}

Host photo-physiology and performance were clearly influenced by Symbiodinium identity, consistent with previous observations of differential photosynthetic performance in different Symbiodinium types (Rowan, 2004; Goulet et al., 2005; Berkelmans and van Oppen, 2006; Loram et al., 2007; Cantin et al., 2009). The homologous S. minutum was the most productive at the whole-symbiosis level, and most beneficial with respect to host growth and pedal laceration, consistent with the carbon flux model of Starzak et al. (2014) for this same host-symbiont combination. In contrast, S. microadriaticum and S. trenchii successfully colonised Aiptasia, reaching relatively high population densities (though not as high as $S$. minutum), but did not contribute substantially to host growth and proliferation. As expected given the inverse relationship between symbiont density and $\mathrm{CO}_{2}$ availability for photosynthesis (Davy and Cook, 2001), the lower density of these two heterologous symbionts relative to the homologous $S$. minutum elevated their photosynthetic rates per cell, but this partial (with S. microadriaticum) or total offset (with S. trenchii) of total symbiosis photosynthesis was insufficient to generate comparable rates of host growth and asexual reproduction. In the case of $S$. microadriaticum, the low symbiont density may partially explain the relatively low photosynthetic rate of anemones colonised by this species compared to that of those colonised by $S$. minutum. However, the situation in the S. trenchiicolonised anemones was different, as their photosynthetic rate was similar to that of the $S$. minutum-colonised anemones. It therefore seems likely that S. trenchii released less of its photosynthate to the host, consistent with previous observations for some members of clade D (Cantin et al., 2009; Jones and Berkelmans, 2010). A further possibility is that S. trenchii is more energetically costly to maintain than $S$. minutum, though this is not supported by the respiration rates measured in my study, which were unaffected by symbiont identity. Previous evidence that some heterologous Symbiodinium taxa are energetically costly when in 
symbiosis with Aiptasia comes from Starzak et al. (2014), who found that S. voratum and type F5.1 induced high rates of host respiration that countered any photosynthetic benefits, and ultimately led to a low P:R ratio and little scope for host growth and reproduction; these authors did not study the impacts of $S$. trenchii, however. The disadvantages of harbouring some members of Symbiodinium clade D on host fitness have been reported previously. For example, when adult and juvenile Acropora sp. associated with Symbiodinium from clade D, it exhibited a decrease in lipid storage, egg size, and overall colony growth, compared to when it hosted Symbiodinium C2/1 (Little et al., 2004; Jones and Berkelmans, 2011).

The behaviour of $S$. microadriaticum and $S$. trenchii is interesting as it is consistent with the 'cheater strategy' exhibited by many parasites, as well as strains of the typically mutualistic Rhizobium in plants, where colonisation is not associated with any discernible benefit for the host (Herre et al., 1999; Sachs and Simms, 2006; Sachs et al., 2010; Sachs et al., 2011a). Previous studies have similarly suggested that several members of Symbiodinium clades A and D are less beneficial than those of, for example, clades B and C (Cantin et al., 2009; Mieog et al., 2009; Jones and Berkelmans, 2010; Stat and Gates, 2011; Lesser et al., 2013). How these symbiont species persisted in Aiptasia is currently unknown, but they might somehow evade the host's immune responses and regulatory pathways, and draw reserves from the host to support their metabolism and growth while providing little or no photosynthetically-fixed carbon to the host in return. Further research on the differential regulation of these symbiont populations and the exchange of metabolites between the partners will help to shed light on this matter. Ultimately, this information will enable us to better understand the long-term implications of harbouring more versus less beneficial symbionts, especially in terms of the ability of coral reefs to recover from bleaching events and adapt as our climate warms. 


\title{
Chapter 3
}

\section{Competitive symbiont uptake in a model cnidarian-dinoflagellate}

\section{symbiosis under heat stress: specificity trumps acquisition of}

\author{
thermo tolerant symbionts
}

\subsection{Introduction}

The symbiosis between anthozoan cnidarians and their photosynthetic dinoflagellate symbionts of the genus Symbiodinium is among the most significant marine mutualisms (Roth, 2014). The genus Symbiodinium is highly diverse, consisting of nine genetic clades (A-I) and numerous sub-clades ('types') (Pochon and Gates, 2010) that display considerable physiological diversity (Schoenberg and Trench 1980b; Fitt et al., 1981; Chang et al., 1983; Iglesias-Prieto et al., 2004; Robison and Warner, 2006; Hennige et al., 2009). Given this, differences in the symbiont community composition inside a host can differentially affect the performance of the overall holobiont (i.e. host plus symbionts), and hence the ability of corals to live in different environments, such as different latitudes, depths, irradiances and temperatures (Buddemeier and Fautin, 1993; Baker, 2001b; Thornhill et al., 2008).

Despite the high diversity of symbionts, host-symbiont associations exhibit some degree of specificity and are nonrandom (Santos et al., 2004; Thornhill et al., 2006; LaJeunesse et al., 2008). Indeed, it is well known that some coral species host multiple symbiont types (Iglesias-Prieto et al., 2004; Sampayo et al., 2007; Silverstein et al., 2012), while others host only one specific symbiont type (LaJeunesse et al., 2003). This specificity depends on the host (Iglesias-Prieto et al., 2004; Little et al., 2004; Thornhill et al., 2006), the symbiont (Banaszak et al., 2000; Loram et al., 2007; Reynolds et al., 2008; Stat et al., 2008; Ragni et al., 2010) and the environment (Santos et al., 2004; Thornhill et al., 2006; LaJeunesse et al., 2008; Bongaerts et al., 2010). The cellular mechanisms involved in determining specificity are still unclear, though cell-cell surface recognition during phagocytosis of the symbiont by 
the host is known to be involved (Rodriguez-Lanetty and Wood-Charlson, 2006; Dunn and Weis, 2009), as is the capacity of different symbiont types to circumvent the host's immune response (Wood-Charlson et al., 2006; Logan et al., 2010b). Furthermore, the competitive interactions between different Symbiodinium types, and the potential for competitive exclusion as a means of establishing a high degree of host-symbiont specificity, have received little attention.

Understanding how host-symbiont specificity arises is not only important for understanding the evolution of particular partnerships, but the potential for corals to form new associations after bleaching events, a dysmbiosis, when corals pale due to the loss of their symbiotic dinoflagellates as a result of elevated seawater temperature. In this latter regard, Buddemeier and Fautin (1993) proposed the "adaptive bleaching hypothesis", which states that bleaching can promote symbiont community change that can be beneficial to the coral host. This could occur through symbiont 'shuffling' (i.e. a change in the relative abundance of the existing symbionts where multiple types co-exist) or 'switching' (i.e. acquisition of new symbionts acquired from the environment), with the newly dominant symbionts being more suited to the new environment. This hypothesis has been tested experimentally, both in the field and laboratory (Baker, 2001b; Kinzie et al., 2001), with widespread evidence for shuffling (Fitt, 2000; Mieog et al., 2007; LaJeunesse et al., 2009; Kemp et al., 2014) but not switching. This lack of evidence for switching suggests that homologous symbionts (i.e. those that regularly form a symbiosis with a particular host) may competitively exclude heterologous symbionts (i.e. those that typically do not form a symbiosis with that species) even if these 'foreign' symbionts manage to evade the host's immune system. Indeed, when hosts are rendered aposymbiotic (i.e. symbiont-free) under laboratory conditions they can typically form new associations when inoculated with a range of homologous or heterologous symbionts, yet such a ready ability to form new, lasting partnerships clearly does not occur in the field.

The sea anemone Exaiptasia pallida (Grajales and Rodríguez, 2014; referred to here after as 'Aiptasia') is widely-adopted model system for the study of reef-building corals and the cnidarian-dinoflagellate symbiosis in general (Weis et al., 2008; Baumgarten et al., 2015). This anemone is distributed throughout the world's tropical seas (Fautin, 2013), and in the Pacific Ocean associates only with Symbiodinium minutum (ITS2 type B1) (Thornhill et al., 2013). Consistent with this high degree of host-symbiont specificity, in the previous work 
(Chapter 2) I demonstrated that, in the laboratory, S. minutum colonises Aiptasia at a much faster rate than members of Symbiodinium species $S$. microadriaticum and $S$. trenchii, while Symbiodinium C3 and S. voratum failed to persist after just a few weeks (Chapter 2). Furthermore, S. minutum formed a more beneficial symbiosis, facilitating more rapid host growth and asexual reproduction, consistent with its higher photosynthetic rate and translocation of fixed carbon to the host (Starzak et al., 2014; Chapter 2). The relationship between Aiptasia and $S$. minutum therefore provides an excellent model for elucidating the mechanisms that promote such a high degree of partner fidelity in the field, despite the ability of this sea anemone to form a range of associations in a laboratory setting.

Here I measured the competitive ability of $S$. minutum, the homologous partner of Aiptasia relative to two heterologous Symbiodinium types, the opportunistic, thermally-tolerant $S$. trenchii (ITS2 type D1a) and S. microadriaticum (ITS2 type A1). More specifically, we tested this competitiveness by: (1) inoculating Aiptasia simultaneously with the homologous and heterologous symbiont types; and (2) challenging homologous and heterologous symbiont types once they had already established a persistent symbiosis with Aiptasia, with exogenously supplied symbionts. Furthermore I explored the effect of elevated temperature on this competitiveness.

\subsection{Materials and Methods}

\subsubsection{Experimental organisms}

All experiments were performed with a clonal culture of the symbiotic sea anemone Exaiptasia pallida (culture ID: NZ1; commonly referred to as Aiptasia) isolated from the Indo-Pacific region. Polyps were maintained in aquaria at $24{ }^{\circ} \mathrm{C}$ under a standard light regime of $80 \mu \mathrm{mol}$ photons $\cdot \mathrm{m}^{-2} \cdot \mathrm{s}^{-1}$ photosynthetically active radiation (PAR) on a 12:12 (light:dark) photoperiod, and fed twice weekly with freshly hatched Artemia nauplii. To generate aposymbiotic (i.e. symbiont-free) anemones, polyps were menthol-bleached as described by (Matthews et al., 2015). This treatment was repeated daily for four weeks or until no symbionts were present, as determined by the absence of chlorophyll fluorescence 
under confocal microscopy (IX81, Olympus New Zealand; $635 \mathrm{~nm}$ laser, 655-755 nm emission filter).

Based on my earlier work (Chapter 2), three species of cultured Symbiodinium were chosen as inoculates (S. minutum, S. microadriaticum and S. trenchii; See Chapter 2, Table 2.1 for details). The algae were sub-cultured from long-term (> 5 years) laboratory stocks and grown in silica-free $\mathrm{f} / 2$ medium (Sigma-Aldrich, Auckland, New Zealand) under the same conditions as the anemones. All cultures were sampled for experimental use during the log phase of growth.

\subsubsection{Simultaneous competitive exposure}

Aposymbiotic anemones $(n=4$ per symbiont mixture, temperature, and time point combination) were inoculated with one of six Symbiodinium mixtures: S. microadriaticum only, S. minutum only, S. trenchii only, S. microadriaticum + S. minutum, S. minutum $+S$. trenchii, or S. microadriaticum $+S$. trenchii. Polyps of similar size (2-3 mm oral disc diameter) were starved for a week then transferred to 400-ml clear plastic jars (one anemone per jar) filled with $0.2 \mu \mathrm{m}$ filtered seawater (FSW; IKA T-10, ThermoFisher Scientific) and allowed to settle for three days. Each algal mixture was diluted in $25 \mathrm{ml} \mathrm{FSW}$ and mixed with one drop of Artemia nauplii, giving a final concentration of $\sim 1 \times 10^{6}$ Symbiodinium cells $\bullet \mathrm{ml}^{-}$ ${ }^{1}$. Using a glass pipette, $1 \mathrm{ml}$ of inoculate was dispensed onto the oral disc of each polyp. Thereafter, anemones were fed once weekly, followed the next day by a water change.

Inoculated anemones were maintained at 24,32 , or $34^{\circ} \mathrm{C}$ under the standard light regime. Polyps were sacrificed one and two weeks post-inoculation to determine the absolute and relative abundances of different Symbiodinium species. Each polyp was homogenized in 500 $\mu \mathrm{FSW}$ and the total volume of the homogenate was determined to a precision of $0.05 \mathrm{ml}$ and centrifuged (Sigma 3-16k) for $5 \mathrm{~min}$ at $400 \mathrm{RCF}$ to separate the algal cells from the anemone tissues. A $100 \mu \mathrm{l}$ sample was removed from the supernatant (host fraction) for protein determination via the Bradford assay (Bradford, 1976) and the remaining supernatant was discarded. The pellet containing the algal cells was re-suspended in $200 \mu$ l guanidinium buffer for DNA extraction and qPCR analysis (see below). 


\subsubsection{Delayed competitive exposure}

Aposymbiotic anemones $(n=6$ per symbiont mixture, temperature, and time point combination) were first inoculated with either homologous $S$. minutum or heterologous $S$. microadriaticum. The inoculated polyps were maintained at $24{ }^{\circ} \mathrm{C}$ until they became densely colonised (minimum of four weeks). The anemones were then challenged via exposure to the alternate Symbiodinium species (i.e. anemones colonised by $S$. minutum were re-inoculated with S. microadriaticum and vice versa), and maintained at 24,32 , or $34{ }^{\circ} \mathrm{C}$ under the standard light regime. Polyps were sacrificed one, two, and four weeks post-challenge to determine the absolute and relative abundances of different Symbiodinium species as describe above.

\subsubsection{Quantitative PCR}

The qPCR methodology was modified from Yamashita et al., (2011). Symbiont DNA was extracted by re-suspending the algal pellet in $200 \mu \mathrm{l}$ guanidinium buffer (50\% guanidinium isothiocyanate, $4.24 \%$ sarkosyl, $2.1 \% \beta$-mercaptoethanol, $10 \mathrm{mM}$ EDTA, $50 \mathrm{mM}$ Tris, $\mathrm{pH}$ 7.6). After extraction for 1 week at $4{ }^{\circ} \mathrm{C}$, the tubes were heated to $72{ }^{\circ} \mathrm{C}$ for $10 \mathrm{~min}$, vortexed several times and centrifuged (16,100 RCF, $5 \mathrm{~min})$. Supernatant $(200 \mu \mathrm{l})$ was transferred to new tubes containing $200 \mu \mathrm{l}$ cold molecular grade isopropanol and stored overnight at $-20{ }^{\circ} \mathrm{C}$ for DNA precipitation. The tubes were then centrifuged (16,100 RCF, $10 \mathrm{~min})$, washed twice in $200 \mu 170 \%$ ethanol, and dried. $50 \mu \mathrm{l}$ of $1 \mathrm{M}$ Tris $(\mathrm{pH}$ 8) were added to each tube and milled without beads at $30 \mathrm{~Hz}$ for $1 \mathrm{~min}$ (TissueLyser LT, Qiagen, Bio-Strategy, New Zealand) to elute the DNA. Samples were stored at $-20^{\circ} \mathrm{C}$ until further analysis.

PCR amplification targeted the partial 18S, ITS1, 5.8S, ITS2 and partial 28S Symbiodinium rDNA region using the forward primer S-DINO (5'- CGCTCCTACCGATTGAGTGA -3') and reverse primer L0 (5'- GCTATCCTGAGRGAAACTTCG -3') (Pawlowski et al., 2001). Reactions were performed using MyTaqTM (Bioline) mix in a total volume of $25 \mu 1$ with the following amplification profile: $95^{\circ} \mathrm{C}$ for $3 \mathrm{~min} ; 35$ cycles of $95^{\circ} \mathrm{C}$ for $30 \mathrm{~s}, 56^{\circ} \mathrm{C}$ for $30 \mathrm{~s}$, and $72{ }^{\circ} \mathrm{C}$ for $30 \mathrm{~s}$; and $72{ }^{\circ} \mathrm{C}$ for $10 \mathrm{~min}$. The PCR products were separately inserted into $E$. coli using the TOPO TA cloning kit (K4530-20, Invitrogen, Thermo Fisher Scientific, NZ) and the $E$. coli were grown on selective LB-agar-ampicillin plates containing $1 \times$ IPTG and 
XGAL (Bioline). DNA was extracted from the appropriate E. coli using the GenElute plasmid miniprep kit (PLN70, Sigma-Aldrich, NZ) and sequenced $(n=4$ samples from each symbiont type) by Macrogen Inc. (Seoul, Korea).

Sequences were aligned using Geneious Pro 4.8.5 (Biomatter Limited, Auckland, NZ) and a BLAST search was performed against the National Centre for Biotechnology Information (NCBI) database in order to identify ITS2 type membership. One sample from each sequenced ITS2 type was chosen to generate qPCR standard curves with 5 serial $\log 10$ dilutions of 1-10,000 ng $\bullet \mathrm{l}^{-1}$ DNA. Reactions were performed on an Applied Biosystems StepOne instrument (Life Technologies) using the powerup SYBR green master mix (Applied Biosystems, Life Technologies, NZ) and clade-specific primers (Yamashita et al., 2011; Table 3.1) in a total volume of $10 \mu \mathrm{l}$ with the following amplification profile: $95^{\circ} \mathrm{C}$ for $10 \mathrm{~min} ; 40$ cycles of $95^{\circ} \mathrm{C}$ for $15 \mathrm{~s}$ and $60^{\circ} \mathrm{C}$ for $1 \mathrm{~min}$.

To confirm cell enumeration by qPCR, one Symbiodinium culture sample of each of the three types was diluted to $9.35 \times 10^{6}$ cells $\cdot \mathrm{ml}^{-1}$ using a haemocytometer (Improved Neubauer, Bright-line), extracted, and amplified. There were six replicates per sample and nine replicate haemocytometer counts per replicate. For the combinations $S$. microadriaticum $+S$. minutum and $S$. minutum + S. trenchii, seven two-culture mixtures were generated at a final concentration of $9.35 \times 10^{6}$ cells $\bullet \mathrm{ml}^{-1}$ with Symbiodinium type ratios ranging from 1:99 to 99:1. Following qPCR, the predicted versus observed symbiont ratios were assessed for accuracy using least-squares linear regression, and the $28 \mathrm{~S}$ copy number per cell was estimated for each type by deduction from the regression lines and comparison with the standard curves that were generated using the purified plasmid DNA for each type (Appendix $3)$. 
Table 3.1. Clade-specific primers: sequences, $T m$ values and amplicon sizes (Yamashita et al., 2011)

\begin{tabular}{clcc}
\hline Name & \multicolumn{1}{c}{ Sequence } & $\begin{array}{c}\text { Tm } \\
\left({ }^{\circ} \mathrm{C}\right)\end{array}$ & $\begin{array}{c}\text { Amplicon } \\
\text { size }(\mathrm{bp})\end{array}$ \\
\hline SymA28S-1F & 5-GAT TGT GGC CTT TAG ACA TAC TAC C-3 & 56.3 & 126 \\
SymA28S-1R & 5-CTC TGA GAG CAA GTA CCG TGC-3 & 56.5 & \\
SymB28S-1F & 5-CAC ATG TCG TGC TGA GAT TGC-3 & 58.9 & 108 \\
SymB28S-1R & 5-CTC GCA TGC TGA GAA ACA CTG-3 & 58.3 & \\
SymD28S-1F & 5-AAT GCT TGT GAG CCC TGG TC-3 & 58.7 & \multirow{2}{*}{114} \\
SymD28S-1R & 5-AAG GCA ATC CTC ATG CGT ATG-3 & 58.7 & \\
\hline
\end{tabular}

\subsubsection{Statistical analysis}

To test the effect of temperature on overall symbiont density, the data were log-transformed to achieve normality and analysed via ANOVA and Tukey post hoc tests with $\alpha=0.05$ in STATISTICA 10 (TIBCO Software, California, USA). Each competitive inoculation trial produced absolute cell counts of two symbiont types per anemone. Each cell was considered a binary outcome measure: either belonging to the first symbiont type or not. These binary data were fit to a logistic regression using the glm function in the base 'stats' $\mathrm{R}$ package ( $\mathrm{R}$ Core Team, 2016). The fixed factors of temperature, time, and their interaction were included and tested against the null. The overall significance of each factor was established with a Wald test from the package 'aod' (Lesnoff and Lancelot, 2012). The interaction term did not improve the model in all but one case, so it was usually dropped. Odds ratios were converted to predicted probabilities of cell membership in the first symbiont type at each temperature or time point and plotted with the package 'ggplot2' (Wickham, 2009). 


\subsection{Results}

\subsubsection{Temperature-induced bleaching and controls}

When colonised by a single Symbiodinium type, there was a significant reduction in symbiont cell density (symbiont cells $\bullet \mathrm{mg}$ host protein ${ }^{-1}$ ) between $24{ }^{\circ} \mathrm{C}$ and $34{ }^{\circ} \mathrm{C}$ (one-way ANOVA, $p<0.05$ for all symbiont types; Fig. 3.1A-C). This pattern was similar in the competition trials (see Appendix 4, Fig. 4A.1). Throughout the experiments there was no detected crosscontamination of symbiont types between treatments; aposymbiotic anemones remained symbiont-free.
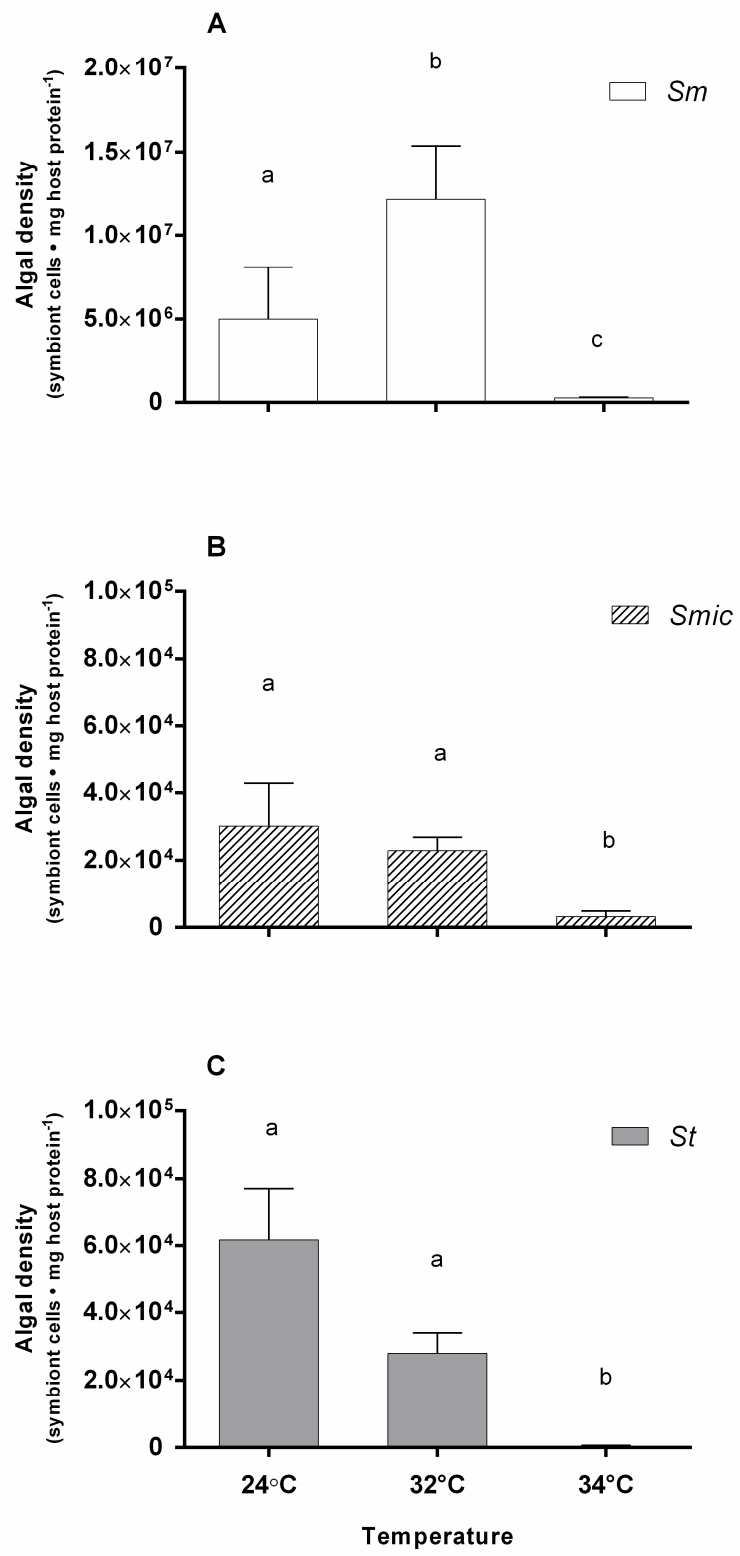

Fig. 3.1. Thermal bleaching in host Aiptasia regardless of symbiont type. Symbiont density (symbiont cells $\cdot \mathrm{mg}$ host protein $^{-1}$ ) in host anemones was quantified two weeks after inoculation for both control $\left(24^{\circ} \mathrm{C}\right)$ and thermal stress (32 and $34{ }^{\circ} \mathrm{C}$ ) conditions (one-way ANOVA, Tukey post hoc). (A) $S m=S$. minutum; (B) Smic = S. microadriaticum; (C) $S t=S$. trenchii. Bars represent means $\pm \mathrm{SE}, \mathrm{n}=4$ anemones per treatment. Different letters above bars indicate significant differences between treatments $(\mathrm{p}<0.05)$. 


\subsubsection{Simultaneous competitive exposure}

Colonisation success varied with symbiont type. The homologous S. minutum consistently outcompeted heterologous $S$. microadriaticum or $S$. trenchii at all time points and temperatures, with an average colonisation success of $>93 \%$ (Fig. 3.2A, B). This pattern was similar in the competition trials (see Appendix 4, Fig. 4A.2). In both trials, temperature and time contributed significantly to the model (Wald test, $p<0.001$ in all cases), and S. minutum colonisation ranged from 93-100 \% (versus S. microadriaticum) or 96-100\% (versus $S$. trenchii).

In the competition experiment between two heterologous cultures (S. microadriaticum and $S$. trenchii), colonisation success varied with temperature (Wald test, $p<0.001$ ) but not with time (Fig. 3.2C). S. microadriaticum colonisation increased with temperature (from 11-15\% colonisation at $24{ }^{\circ} \mathrm{C}$, to at $35-48 \%$ colonisation at $32{ }^{\circ} \mathrm{C}$, and $57-80 \%$ colonisation at 34 $\left.{ }^{\circ} \mathrm{C}\right)$. By necessity, S. trenchii showed the opposite pattern: its relative percentage inside the anemone decreased as the temperature increased.

\subsubsection{Delayed competitive exposure}

When anemones were challenged with homologous S. minutum after first forming a stable association with heterologous $S$. microadriaticum, $S$. minutum was able to partially colonise the anemone, with a maximum colonisation success of $28 \%$ (Fig. 3.3A, B, C). Here, temperature, time, and their interaction contributed significantly to the model (Wald test; $p$ $<0.001$ in all cases). Given the interaction, it was easier to consider the average probability of a given cell belonging to a given type at each temperature or time point. Time had the greatest impact, with the average probability of a cell belonging to $S$. minutum increasing from week $1(8 \%)$ to week $2(11 \%)$ to week $4(28 \%)$. In the alternate case, when anemones had already formed a stable symbiosis with homologous $S$. minutum, heterologous $S$. microadriaticum did not manage to colonise the polyps in appreciable amounts at any temperature or time point $(<1 \%$ colonisation in all cases; Fig. 3.3D, E, F). 


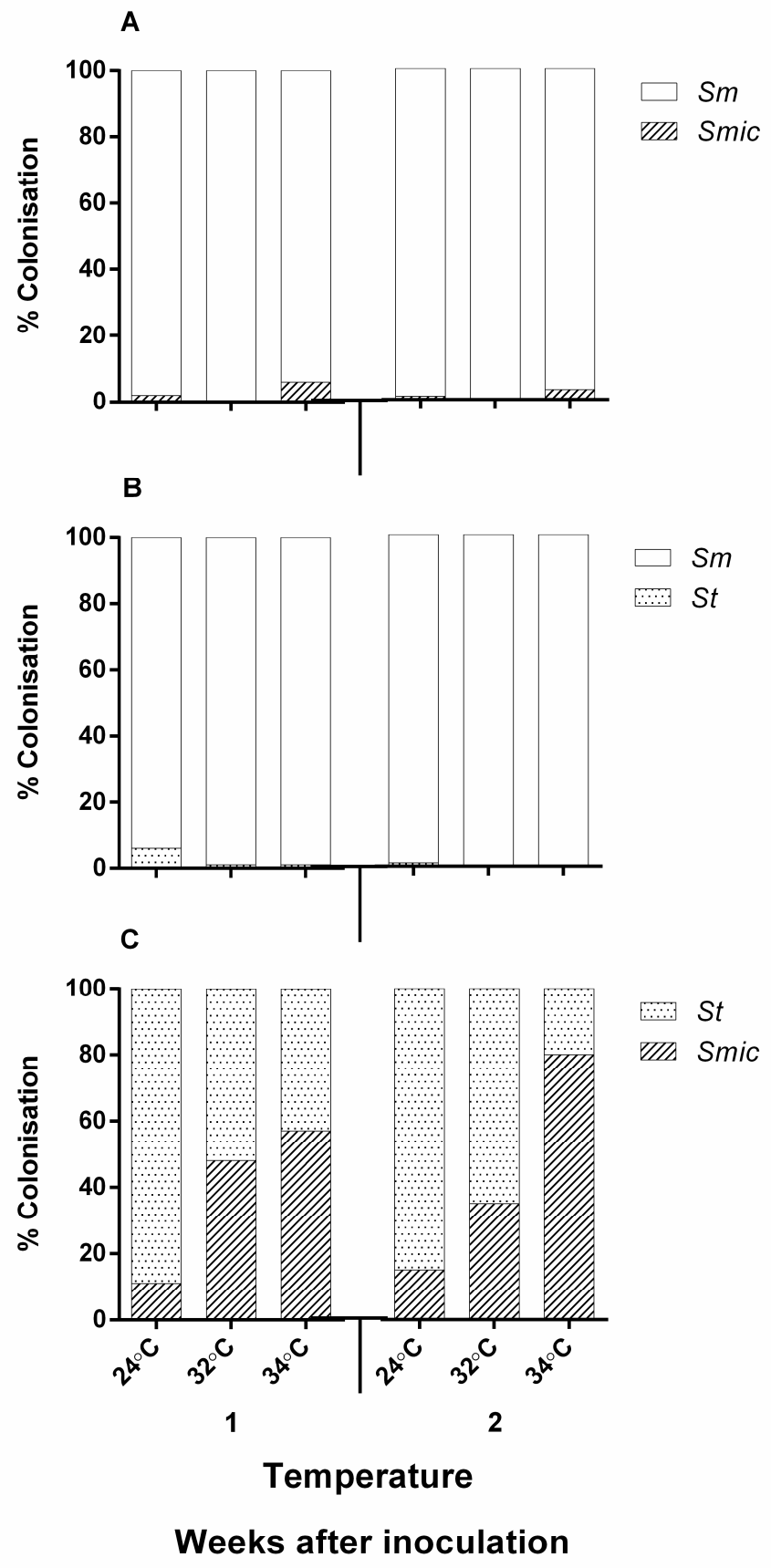

Fig. 3.2. Symbiont success in simultaneous competitive exposure varied with Symbiodinium species and temperature. Percentage colonisation success of the different Symbiodinium species simultaneously being inoculated in Aiptasia host was quantified one and two weeks after inoculation under both control $\left(24^{\circ} \mathrm{C}\right)$ and thermal stress $\left(32\right.$ and $\left.34{ }^{\circ} \mathrm{C}\right)$ conditions ( $n=4$ anemones per treatment per time point; Wald test). (A) S. microadriaticum $+S$. minutum (B) S. minutum + S. trenchii (C) S. microadriaticum + S. trenchii. $S m=$ Symbiodinium minutum; St $=S$. trenchii; Smic $=S$. microadriaticum. For statistical description, see the main text. 


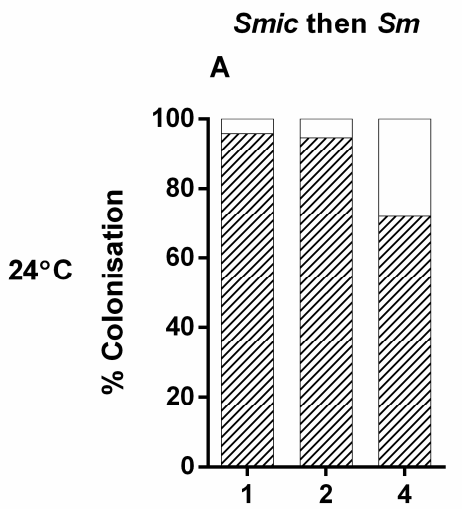

B

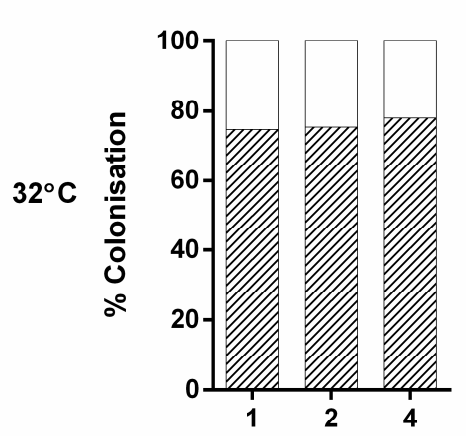

C

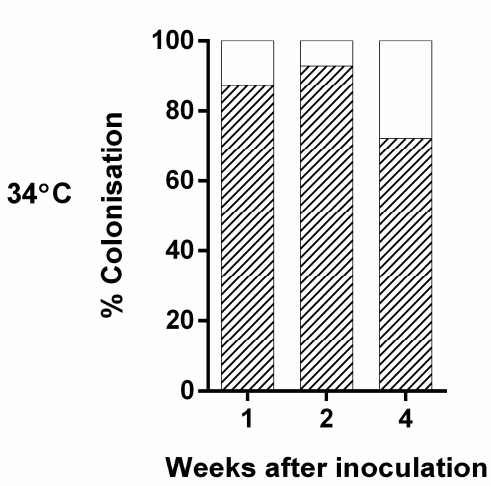

Sm then Smic

D

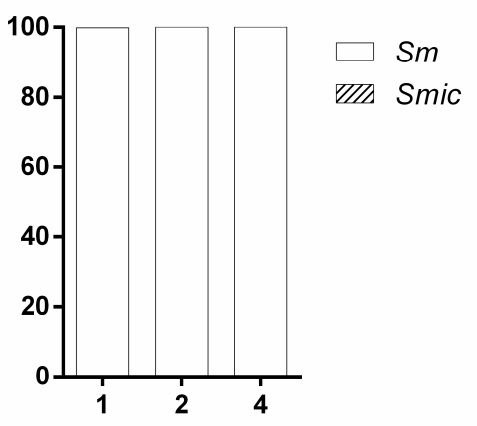

$E$

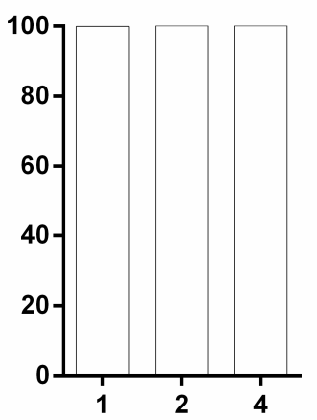

$\mathbf{F}$

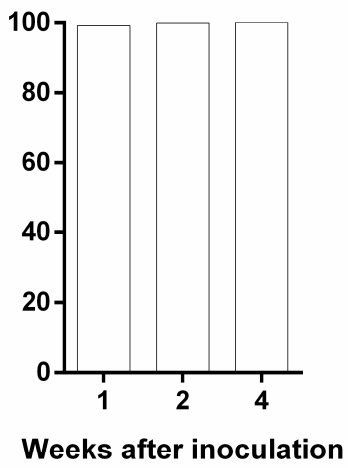

Fig. 3.3. Symbiont specificity is the key factor in determining symbiont success in delayed competitive exposure. Percentage colonisation success of different Symbiodinium species being inoculated in already populated Aiptasia host was quantified one, two and four weeks after inoculation under both control $\left(24{ }^{\circ} \mathrm{C}\right)$ and thermal stress (32 and $34{ }^{\circ} \mathrm{C}$ ) conditions ( $\mathrm{n}=6$ anemones per treatment per time point; Wald test). (A) Anemones populated with $S$. microadriaticum then challenged with $S$. minutum at $24{ }^{\circ} \mathrm{C}(\mathbf{B})$ at $32{ }^{\circ} \mathrm{C}(\mathbf{C})$ at $34{ }^{\circ} \mathrm{C}$; (D) Anemones populated with $S$. minutum then challenged with $S$. microadriaticum at $24{ }^{\circ} \mathrm{C}(\mathbf{E})$ at $32{ }^{\circ} \mathrm{C}(\mathbf{F})$ at $34^{\circ} \mathrm{C} . \mathrm{Sm}=$ Symbiodinium minutum; Smic $=$ S. microadriaticum. For statistical description, see the main text. 


\subsection{Discussion}

\subsubsection{Simultaneous competitive exposure}

This study is the first to investigate the effect of thermal stress on the competitive abilities of different Symbiodinium species in Aiptasia model system. The observed competitive dominance of $S$. minutum in paired simultaneous competition experiments at control temperatures was expected, given that it occurs in Aiptasia from Hawaii in nature, to the exclusion of other symbiont types (Thornhill et al., 2013). However my hypothesis that $S$. minutum would be outcompeted by other species under elevated temperature conditions was refuted by my findings. My prediction was based in part on the behaviour of the three Symbiodinium species in culture and on performance of studies in corals that have demonstrated symbiont shuffling, at least in the short term, after temperature stress. Specifically, S. minutum exhibits thermal sensitivity in culture (Suggett et al., 2008; Hawkins and Davy, 2012) whereas S. microadriaticum and S. trenchii are more thermally robust (Banaszak et al., 2000; Berkelmans and van Oppen, 2006; Reynolds et al., 2008; Ragni et al., 2010). Given these physiological differences, I expected to see a shift away from an $S$. minutum -dominated population and towards an S. microadriaticum- and/or S. trenchiidominated population. Such shuffling of the resident symbiont population in response to thermal stress has been observed previously in the coral Acropora millepora in the field where the symbiont community shifted from the thermally sensitive $\mathrm{C} 2$ to the thermally tolerant D (Berkelmans and van Oppen, 2006; Jones et al., 2008). My results highlight the peril of extrapolating from the physiological performance of an algal species in culture to its performance in hospite, where its interaction with its host may influence its performance and health. Indeed, previous metabolomics and proteomic work in Aiptasia indicates that the host is impacted by thermal stress before its symbiotic $S$. minutum, suggesting that this symbiont type is thermally robust in hospite (Hillyer et al., 2016; Oakley et al., 2017. See Appendix 5).

The outcome of competition experiments between S. trenchii and S. microadriaticum varied with temperature (Fig. 3.2C), with S. trenchii dominating at 24 and $32{ }^{\circ} \mathrm{C}$ and $S$. microadriaticum at $34{ }^{\circ} \mathrm{C}$. Both types exhibited reductions in cell density in hosts at elevated temperature, consistent with thermal stress of the symbiont and/or host. The decrease in 
competitive dominance of $S$. trenchii at the highest temperature was unexpected, given the many studies demonstrating its thermal resistance in recent years (Berkelmans and van Oppen, 2006; LaJeunesse et al., 2009; Kemp et al., 2014; Keshavmurthy et al., 2014; Lee et al., 2016). While both of these symbiont species exhibit distinct physiological tolerances and are able to persist in thermally-stressed corals (Thornhill et al., 2006; LaJeunesse et al., 2009; Mieog et al., 2009; Oliver and Palumbi, 2011; Kemp et al., 2014), these symbiont species also show lower rates of carbon fixation and photosynthetically-fixed carbon translocation to the host (Cantin et al., 2009; Mieog et al., 2009; Jones and Berkelmans, 2010; Leal et al., 2015). For example a study by Stat et al. (2008) showed that when colonies of Acropora cytherea harboured Symbiodinium clade A, they exhibit suboptimal health states and an increased incidence of disease, compared with corals harbouring clade C symbionts. Similar observations have been made in colonies and juveniles containing clade D symbionts (Little et al., 2004; Cantin et al., 2009; Stat and Gates, 2011).

\subsubsection{Delayed competitive exposure}

The ability of the homologous $S$. minutum to maintain its dominance once it had fully established a symbiosis with Aiptasia was marked, with no evidence for the heterologous $S$. microadriaticum being able to establish a symbiosis even in a limited capacity, at any temperature. This was in contrast to the outcome of $S$. microadriaticum-containing Aiptasia challenged with $S$. minutum, where partial colonisation by the homologous symbiont was apparent. A similar pattern was reported by Belda-Baillie et al. (2002), who showed that Aiptasia containing heterologous $S$. microadriaticum could subsequently form a symbiosis with the homologous $S$. minutum, while anemones already colonised by $S$. minutum could not form a symbiosis with symbionts from clades A or C. It is notable however, that four weeks in my delayed competitive exposure experiment, the homologous symbiont made up just $25 \%$ of the symbiont population. Longer-term experiments designed to determine if and when $S$. minutum takes over as the numerically dominant symbiont should be carried out in the future.

This specificity may not only arise from symbiont-symbiont competitiveness, however, but from the overall physiological performance and fitness of the holobiont (i.e. the whole symbiosis). In particular, our previous work with Aiptasia has shown that, even though the 
host can establish a symbiosis with $S$. microadriaticum or S. trenchii in isolation, these symbiont species confer less benefit to the host than does S. minutum (Chapter 2). More specifically, the anemone grew at least 4.5-times faster and produced twice more pedal lacerates when in symbiosis with S. minutum. Furthermore, Matthews et al. (2017) showed that when Aiptasia is colonised with S. trenchii, there is an up-regulation of the host's innate immune and oxidative stress responses, as well as changes to its metabolite profile suggesting that its energy reserves are compromised. Such events likely contribute to the high degree of host-symbiont fidelity seen in this symbiosis, and may well contribute to the relatively weak colonisation success and reduced competitiveness of the heterologous symbionts compared to the homologous $S$. minutum in my experiment, even under thermal stress.

\subsubsection{Ecological implications}

The potential for symbiotic cnidarians to change their dinoflagellate partners as an adaptive mechanism to response to global climate change continues to intrigue researchers (Goulet, 2006; Baker and Romanski, 2007; Oliver and Palumbi, 2011; Kemp et al., 2014; Núñez-Pons et al., 2017). However, host-symbiont specificity may constrain the potential for partner switching, with corals and other cnidarians being limited to the one or few algal partners with which they regularly form an association (Goulet, 2006; Poland and Coffroth, 2017). However, such partner fidelity might not be disadvantageous (Putnam et al., 2012). Putnam and coworkers (2012) hypothesized that the relationship between a specialist cnidarian and its symbionts may be the outcome of stringent coevolution that has resulted in an integrated and functionally optimal mutualism that can persist under both stable and stressful conditions, whereas "symbiotic entrepreneurialism" of generalists may result in competitive interactions between symbionts that ultimately impair function and destabilize the symbiosis. For example, the high degree of host-symbiont specificity between corals of the genus Porites and their symbionts is believed to confer considerable ecological benefit when compared to more generalist genera such as Acropora and Pocillopora (Loya et al., 2001; Fabricius et al., 2011; Van Woesik et al., 2011; Putnam et al., 2012). In this same way, the widespread ecological success of Aiptasia across the Indo-Pacific region could be attributed, in part, to its high degree of partner fidelity, maintained through the strongly competitive nature of $S$. minutum when in symbiosis with this host. Moreover, even though this symbiosis exhibits 
Chapter 3

bleaching at high temperature, the continued dominance of $S$. minutum suggests that Aiptasia will retain its fidelity for this symbiont even as the climate warms. Whether S. minutum continues to offer the greatest ecological benefit to Aiptasia under these changing conditions warrants further study. Likewise, whether events in specialist reef corals mirror those seen in the model Aiptasia system, at both ambient and elevated temperatures, awaits confirmation. Such information will better enable us to predict and manage the responses of reef corals to climate change. 


\section{Chapter 4}

\section{Host cell apoptosis and the regulation of different symbiont species in the cnidarian-dinoflagellate symbiosis}

\subsection{Introduction}

Among the most significant marine symbioses are those between anthozoan cnidarians and their photosynthetic dinoflagellates of the genus Symbiodinium (Roth, 2014). This intimate relationship, in which symbionts live within the host's gastrodermal cells, where they are bound by a vacuolar membrane of animal origin, significantly contributes to the success of the coral reef ecosystem (Wakefield and Kempf, 2001; Douglas, 2010; Wang et al., 2012). Symbionts provide the host with photosynthetically fixed carbon which supports the host's respiration, growth and reproduction (Muscatine, 1990), and in return the cnidarian host provides inorganic carbon, nitrogen and phosphorus, a high-light environment, and refuge from herbivory to the dinoflagellate symbiont (Furla et al., 2005).

The genus Symbiodinium is genetically and physiologically diverse, containing a large number of formally recognized species, as well as a range of other phylotypes belonging to molecular clades A-I (Fitt et al., 1981; Robison and Warner, 2006; Hennige et al., 2009; Pochon and Gates, 2010). A different symbiont community composition inside a host can therefore significantly affect the performance of the overall symbiosis (the 'holobiont') (Iglesias-Prieto et al., 2004; Starzak et al., 2014; Leal et al., 2015). Despite the high diversity of symbionts, however, host-symbiont associations exhibit some degree of specificity, being non-random (Santos et al., 2004; Thornhill et al., 2006; LaJeunesse et al., 2008). Indeed, several colonisation studies have shown differences in the ability of different symbiont phylotypes or species to proliferate inside the host, with algae originally isolated from the same host species being the most successful (Kinzie \& Chee 1979; Schoenberg \& Trench 
1980; Davy et al. 1997; Belda-Baillie et al. 2002; Harii et al. 2009; Starzak et al. 2014; Chapter 2 of this thesis).

Host-symbiont recognition is key for the successful establishment and persistence of a symbiosis. This involves a cascade of molecular signalling between the host cnidarian and the symbiont algae, referred to as 'the winnowing' (Nyholm and McFall-Ngai, 2004). Winnowing processes can be divided into pre- and post-phagocytic events. It has been suggested that lectin-glycan interactions are important for pre-phagocytic recognition in the cnidarian-dinoflagellate symbiosis (Lin et al., 2000; Wood-Charlson et al., 2006; Logan et al., 2010a), while post-phagocytic winnowing processes include the manipulation of the cnidarian's innate immune signalling pathways by the symbionts, so enabling them to avoid intracellular attack (Karakashian and Rudzinska, 1981; Chen et al., 2004, 2005; Hong et al., 2009; Venn et al., 2009; Neubauer et al., 2017). The host is therefore able to sort and regulate its symbiont population using mechanisms such as degradation of symbionts by the host cell (Titlyanov et al., 1996), expulsion of symbionts (Hoegh-Guldberg et al., 1987; HoeghGuldberg and Smith, 1989a; Stimson and Kinzie, 1991; McCloskey et al., 1996) and host cell apoptosis (Dunn and Weis, 2009). However, though there has been some recent progress in the field, we still know relatively little of the processes involved in winnowing.

Here I further explore the role of host cell apoptosis in the post-phagocytic winnowing using two different methods for measuring caspase activity, a key component of apoptosis. Caspases are a conserved family of cysteine-aspartic proteases that play an essential role in programmed cell death and inflammation, and their activation ensures degradation of cellular components in a controlled manner (Thornberry and Lazebnik, 1998). Specifically, I measured the influence of Symbiodinium species on host cell apoptosis by measuring caspase-3 enzyme activity both in situ via confocal microscopy and after extraction of host tissues via an assay. I challenged the sea anemone Exaiptasia pallida (Grajales and Rodríguez, 2014; commonly referred to as 'Aiptasia'), a widely-adopted model system for the study of cnidarian-dinoflagellate symbiosis (Weis et al., 2008; Baumgarten et al., 2015), with four different Symbiodinium species, including the homologous symbiont Symbiodinium minutum (ITS2 phylotype B1; Thornhill et al., 2013), the free-living and relatively heterotrophic Symbiodinium voratum (ITS2 clade E; Jeong et al. 2012), and the thermallytolerant but opportunistic Symbiodinium microadriaticum and Symbiodinium trenchii (ITS2 
phylotypes A1 and D1a, respectively; Schoenberg and Trench, 1980c; Pettay and LaJeunesse, 2009). I hypothesized that anemones challenged with non-native (heterologous) symbionts will show relatively high host cell apoptosis and therefore high caspase-3 activity, as a way of removing inappropriate symbionts, while anemones challenged with their regular (homologous) symbionts will exhibit lower apoptotic activity.

\subsection{Materials and Methods}

\subsubsection{Experimental organisms}

A clonal culture of the symbiotic sea anemone Exaiptasia pallida (culture ID: NZ1; commonly referred to as 'Aiptasia'), originally from the Indo-Pacific region, was grown in the lab at $25{ }^{\circ} \mathrm{C}$ and an irradiance of $80 \mu \mathrm{mol}$ photons $\bullet \mathrm{m}^{-2} \bullet \mathrm{s}^{-1}$ (12-h light/12-h dark), and fed twice weekly with freshly hatched Artemia nauplii. To generate aposymbiotic (i.e. symbiont-free) anemones, animals were incubated in a solution of $0.27 \mathrm{mM}$ menthol/FSW (0.22 $\mu \mathrm{m}$-filtered seawater) for $8 \mathrm{~h}$, after which the menthol/FSW was removed and the anemones were incubated in $5 \mu \mathrm{M}$ DCMU/FSW (3-(3,4-dichlorophenyl)-1,1-dimethylurea) overnight as described by Matthews et al. (2015). This treatment was repeated daily for four weeks or until no symbionts were present, as determined by confocal microscopy (IX81, Olympus New Zealand; $635 \mathrm{~nm}$ laser, 655-755 nm emission filter).

Based on my previous work (Chapter 2), four clades of cultured symbiotic dinoflagellates were chosen as inoculates, representing different rates of host colonisation and symbiotic lifestyles (S. minutum, S. microadriaticum, S. trenchii and S. voratum; See Chapter 2 Table 2.1 for details). The algae were sub-cultured from long-term (> 5 years) laboratory stocks and grown in silica-free $\mathrm{f} / 2$ medium (Sigma-Aldrich, Auckland, New Zealand) at $25{ }^{\circ} \mathrm{C}$ and an irradiance of $80 \mu \mathrm{mol}$ photons $\bullet \mathrm{m}^{-2} \cdot \mathrm{s}^{-1}$, on a 12-h light/12-h dark regime. All cultures were sampled for experimental use during the log phase of growth. 


\subsubsection{Inoculation of aposymbiotic sea anemones with symbionts}

Following one week of starvation, a total of 188 aposymbiotic anemones $(n=40$ anemones for each Symbiodinium species; $n=24$ uninoculated anemones as a negative control; $n=4$ uninoculated anemones exposed to colchicine as positive control) of similar size (2-3 mm oral disc diameter) were transferred to $400 \mathrm{ml}$ clear plastic jars (one anemone per jar) filled with FSW and allowed to settle for three days. Each Symbiodinium culture was diluted in 25 ml FSW and one drop of Artemia suspension added, to give a final concentration of $\sim 2 \times 10^{6}$ Symbiodinium cells $/ \mathrm{ml}$. The anemones $(\mathrm{n}=10$ per treatment per time point) were inoculated by pipetting $1 \mathrm{ml}$ of this dinoflagellate suspension onto the oral disc of each anemone, with a glass pipette. Colonisation was then monitored over a period of eight weeks, with sampling at 1, 7, 14 and 28 days post-inoculation. During this time, the anemones were fed twice weekly with Artemia nauplii, with each feeding followed by a water change. Seawater temperature was maintained at $25{ }^{\circ} \mathrm{C}$ in a controlled temperature room, and irradiance was held at $\sim 80$ $\mu \mathrm{mol}$ photons $\bullet \mathrm{m}^{-2} \bullet \mathrm{s}^{-1}$ on a 12-h light/12-h dark cycle.

\subsubsection{Real-time visualisation of caspase synthesis in Aiptasia}

To visualise the effect of symbiont type on host cell apoptosis, caspase-like enzyme activity was detected in situ using the FLICA dye (Image-iT ${ }^{\mathrm{TM}}$ detection kit I35106, Molecular Probes, Eugene, OR, USA) which contains a fluorescent affinity label and interacts with the enzymatic reaction centre of an activated caspase-3 via the recognition sequence FAMDEVD-FMK. At each time point, three randomly selected anemones from each treatment were transferred to a 12-well plate containing $1 X$ FLICA reagent in relaxation solution (50\% $0.37 \mathrm{M} \mathrm{MgCl}_{2}$ in $\mathrm{dH}_{2} \mathrm{O}$ and $50 \% \mathrm{FSW}$ ). Anemones were incubated in the dark for a total of $90 \mathrm{~min}$. After $60 \mathrm{~min}$ of this incubation period, Hoechst 33342 stain was added ( $1 \mu \mathrm{M}$ final) to label host cell nuclei and aid visualisation of gastrodermal tissue. Anemones were then washed twice to remove excess dye and transferred to a glass-bottom fluorodish (World Precision Instruments). A drop of relaxation solution containing 1.5\% agar was added and randomly selected tentacles were visualised using an Olympus Fluoview FV-1000 inverted confocal LSM (IX81, Olympus New Zealand). Caspase-3 fluorescence was detected at 510$530 \mathrm{~nm}$, with excitation provided by a $473 \mathrm{~nm}$ diode laser. Hoechst 33342 fluorescence was 
detected using a DAPI filter (430-460 nm) and $405 \mathrm{~nm}$ excitation, and symbiont chlorophyll autofluorescence was detected using a $635 \mathrm{~nm}$ laser and 655-755 nm emission filter. Z-stack images were generated (section thickness $<10 \mu \mathrm{m}$ ) and the sections were then summed into a single photo per tentacle.

\subsubsection{Preparation of tissue homogenate and quantification of symbiont density}

Randomly chosen anemones ( $\mathrm{n}=7$ per treatment at each time-point) were rinsed separately with relaxation solution, immediately flash-frozen in liquid nitrogen, and stored at $-80^{\circ} \mathrm{C}$. Samples were then thawed on ice and homogenized separately (IKA T-10 tissue lyser, ThermoFisher Scientific) in $400 \mu 1$ homogenisation buffer (50 mM potassium phosphate, $\mathrm{pH}$ 7.8, $1 \mathrm{mM}$ EDTA). The homogenate was centrifuged (Sigma 3-16k) for $10 \mathrm{~min}$ at $700 \times \mathrm{g}$ to separate algal cells from anemone tissue and the supernatant (host fraction) was transferred to a new tube and centrifuged at high speed $(14000 \times \mathrm{g}$ for $10 \mathrm{~min})$ to remove debris. A $40 \mu 1$ aliquot was then taken for protein determination and the remaining supernatant immediately flash-frozen in liquid nitrogen and stored at $-80^{\circ} \mathrm{C}$. The pellet containing the dinoflagellate cells was re-suspended in $200 \mu \mathrm{l} \mathrm{FSW}$. A 50- $\mu \mathrm{l}$ aliquot was added to $50 \mu \mathrm{l}$ dimethyl sulfoxide buffer for DNA sequencing (see below), and the remaining $150 \mu \mathrm{l}$ were used for algal cell counts. The samples were stored at $-20{ }^{\circ} \mathrm{C}$ until further analysis. Caspase- 3 activity was determined using a colorimetric assay kit (see below), host protein content was determined via the Bradford assay (Bradford, 1976) and cell counting was performed with a haemocytometer (Improved Neubauer) with six replicate counts per sample. Symbiont cell density (symbionts per mg protein) was then calculated.

\subsubsection{Assessment of host caspase-like enzyme activity in Aiptasia}

Host caspase-3 activity per treatment at each time-point was quantified using a colorimetric assay kit (product code: CASP-3-C, Sigma-Aldrich, Auckland, New Zealand) with the substrates Ac-Asp-Glu-Val-Asp-p-nitroanilide (Ac-DEVD-pNA). Caspase enzymes cleave the terminal aspartic acid residue in the substrate with a specificity determined by the identity of the preceding three amino acid residues. Duplicates of $50 \mu \mathrm{l}$ aliquots were analysed for caspase-like activity according to the manufacturer's instructions. Briefly, $50 \mu \mathrm{l}$ anemone 
host fraction were added to $40 \mu \mathrm{l}$ "assay buffer" [20 $\mathrm{mM}$ 4-(2-hydroxyethyl)-1piperazineethanesulfonic acid (HEPES), pH 7.4, 2 mM EDTA, 0.1\% 3-[(3-cholamidopropyl)dimethylammonio]-1-propanesulfonate (CHAPS), $5 \mathrm{mM}$ dithiothreitol (DTT)] and $10 \mu \mathrm{l}$ caspase substrate $(2 \mathrm{mM})$. Sample blanks were prepared simultaneously, to control for nonspecific binding, by adding $50 \mu \mathrm{l}$ host fraction to $50 \mu \mathrm{l}$ assay buffer only. Three positive controls were also prepared: (1) To confirm the insertion of the dye, $5 \mu$ l caspase-3 $(5 \mu \mathrm{g} / \mathrm{ml}$ containing $1 \mathrm{mg} / \mathrm{ml} \mathrm{BSA}$ ) were added to $10 \mu \mathrm{l}$ caspase substrate; (2) to confirm the effectiveness of the kit, aposymbiotic anemones were incubated with colchicine $0.05 \% \mathrm{w} / \mathrm{v}$ for 24 hours before $10 \mu \mathrm{l}$ caspase substrate were added to $50 \mu \mathrm{l}$ sample and $40 \mu \mathrm{l}$ assay buffer; and (3) to confirm the specificity of the kit, $10 \mu$ l caspase-3 inhibitor (DEVD-CHO, $200 \mu \mathrm{M}$ ) were added to $10 \mu \mathrm{l}$ caspase substrate, $50 \mu \mathrm{l}$ sample treated with colchicine and 40 $\mu \mathrm{l}$ assay buffer. Samples and controls were incubated for $3 \mathrm{~h}$ at $37^{\circ} \mathrm{C}$. The two latter controls showed that colchicine treatment caused an elevation in caspase-like enzyme activity that was reversed in the presence of caspase inhibitor (Fig. 4.1; one-way ANOVA, Tukey post hoc, $P$ $<0.002)$. Caspase activity was normalized to $\mathrm{mg}$ host protein and was also tested for a correlation with symbiont density to assess the effect of symbiont density on host-cell apoptosis. 


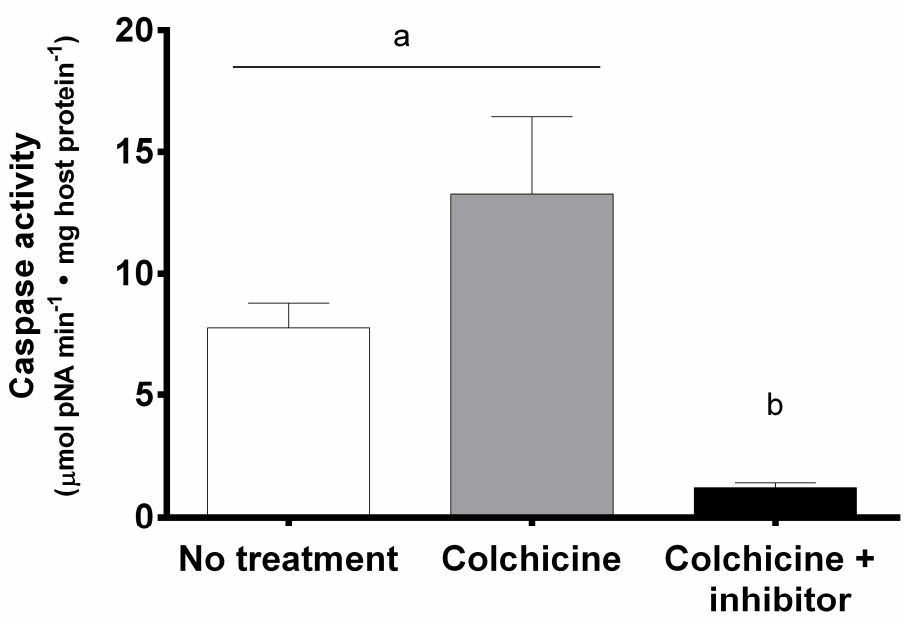

Fig. 4.1. Positive and negative controls of caspase-like enzyme activity in aposymbiotic specimens of the sea anemone Aiptasia. Caspase-like enzyme activity ( $\mu$ mol pNA min ${ }^{-1}$ • mg host protein $^{-1}$ ) was quantified 28 days after inoculation. Anemones either had no treatment, or were treated with the microtubule inhibitor and apoptosis inducer colchicine $(0.05 \% \mathrm{w} / \mathrm{v}$ for $24 \mathrm{~h})$, in the presence or absence of a caspase inhibitor. Values are mean $\pm \mathrm{SE}$ and letters indicate bars that are significantly different from each other within each parameter $(\mathrm{n}=3$ per each treatment, two-way ANOVA, Tukey post hoc).

\subsubsection{DNA sequencing}

To confirm that symbiont populations in animals matched the populations used for inoculation, symbiont type was verified for each culture at the start of the experiment and a subset of inoculated anemone samples at the end of the experiment ( $n=3$ samples per culture or anemone treatment). Symbiodinium samples containing DMSO (see above) were resuspended, and $10 \mu \mathrm{l}$ of each sample added to a new tube containing $50 \mathrm{mg}$ glass beads (G1152-10G, Sigma-Aldrich) and placed in a TissueLyser (Qiagen, Bio-Strategy) bead beater for $1 \mathrm{~min}$ at $50 \mathrm{~Hz}$. Ninety microliters of $\mathrm{ddH}_{2} \mathrm{O}$ were added, and lysed cell material was pelleted at $16,100 \times \mathrm{g}$ for $10 \mathrm{~min}$ at $4{ }^{\circ} \mathrm{C}$. A $50 \mu \mathrm{l}$-aliquot of the resulting supernatant was transferred to a new tube containing $50 \mu \mathrm{l}$ cold molecular-grade isopropanol, and samples were re-pelleted as described above. Samples were washed twice in $200 \mu 170 \%$ ethanol, centrifuged and then dried at room temperature. Fifty microliters of $1 \mathrm{M}$ Tris $(\mathrm{pH} 8)$ were 
added to each tube and samples were further extracted by bead beating at $30 \mathrm{~Hz}$ for $1 \mathrm{~min}$ and then stored at $-20{ }^{\circ} \mathrm{C}$ until further analysis. Polymerase chain reaction (PCR) was performed using the following Symbiodinium-specific ITS2 (internal transcribed spacer region 2) primers: forward primer (ITS2infor) 5'-GAATTGCAGA ACTCCGTG-3'; reverse primer (ITS2CLAMP) 5'-CGCCCGCCGC GCCCCGCGCC CGTCCCGCCG CCCCCGCCC GGGATCCATA TGCTTAAGTT CAGCGGGT-3'. Reactions were performed using the MyTaq $^{\mathrm{TM}}$ Mix in a total volume of $25 \mu \mathrm{l}$ with an amplification profile consisting of: 1 cycle of 3 min $95{ }^{\circ} \mathrm{C}$; 40 cycles of 15 s $95{ }^{\circ} \mathrm{C}, 15$ s $56{ }^{\circ} \mathrm{C}$ and $10 \mathrm{~s} 72{ }^{\circ} \mathrm{C}$; and a final hold temperature of $4{ }^{\circ} \mathrm{C}$. PCR products were sequenced by Macrogen Inc. (Seoul, South Korea). Sequences were aligned using Geneious Pro 4.8.5 and a BLAST search was performed in the National Centre for Biotechnology Information (NCBI) database to identify the sequences. In all cases, the expected genotypes were present both at the beginning and end of the experiment. Multiple genotypes were never detected in any sample.

\subsubsection{Statistical analysis}

A Kruskal-Wallis test followed by the Mann-Whitney post hoc test were performed on algal density data using STATISTICA 10. To avoid a Type I error of incorrectly rejecting the null hypothesis, a Bonferroni correction was performed on both the Kruskal-Willis and MannWhitney tests. Analysis of Variance (ANOVA) followed by the Tukey post hoc test, as well as the Pearson correlation test were performed on caspase activity data using STATISTICA 10 and SPSS to confirm whether algal density has an effect on caspase activity. Natural log transformation $(\mathrm{Ln})$ was conducted on caspase activity per unit host protein data to achieve a normal distribution. Results are expressed as mean \pm standard error (SE).

\subsection{Results}

\subsubsection{Colonisation success}

All Symbiodinium species initially colonised Aiptasia with no cross-contamination between treatments. Aposymbiotic anemones remained symbiont-free throughout the experiment. 
Symbiont cell density (algal cells $\cdot \mathrm{mg}$ host protein $^{-1}$ ) was significantly different between anemones inoculated with different Symbiodinium species (Fig. 4.2; Kruskal-Wallis with Bonferroni correction, $P<0.01$ for all days except Day 1). The homologous S. minutum was significantly more successful at colonising hosts than the other species (Kruskal-Wallis, Mann-Whitney post hoc, $\mathrm{P}<0.0022)$, reaching a density of $4.47 \times 10^{6} \pm 2.89 \times 10^{5}$ cells $\bullet \mathrm{mg}$ protein $^{-1}$ at day 28. S. microadriaticum was the second most successful species, with a density of $3.15 \times 10^{5} \pm 1.54 \times 10^{5}$ cells $\bullet \mathrm{mg}$ protein $^{-1}$ at day 28 . S. trenchii struggled to colonise the host and reached a peak of only $1.89 \times 10^{4} \pm 1.81 \times 10^{3}$ cells $\cdot \mathrm{mg}$ protein $^{-1}$ at day 14 , while $S$. voratum initially colonised the host but after one week its density declined dramatically, such that by day 7 and thereafter, no symbionts were observed (Fig. 4.2).

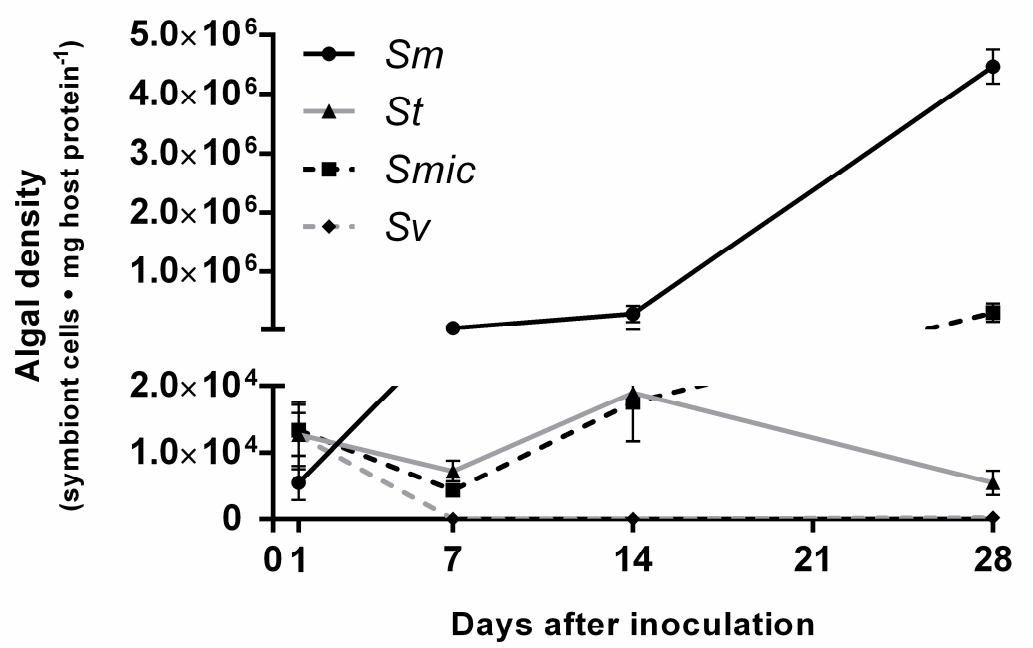

Fig. 4.2. Colonisation success in Aiptasia varied with symbiont type. Symbiont density (symbiont cells $\cdot \mathrm{mg}$ host protein $^{-1}$ ) in host anemones was quantified for 28 days after inoculation ( $\mathrm{n}=7$ anemones per treatment per time point; Kruskal-Wallis, Mann-Whitney post hoc, both with Bonferroni correction). Sm = Symbiodinium minutum; St $=S$. trenchii; Smic $=S$. microadriaticum $; v=S$. voratum. Points are means \pm SE. The density of the symbiont species $S$. voratum was not detected after Day 7 and therefore was not analysed this way (see Statistical Analysis above). 


\subsubsection{Caspase-like enzyme activity}

Caspase activity ( $\mu \mathrm{mol} \mathrm{pNA} \min ^{-1} \cdot \mathrm{mg}$ host protein $^{-1}$ ) was significantly different between anemones inoculated with different Symbiodinium types (Fig. 4.3A\&B; two-way ANOVA, $\left.\mathrm{F}_{4,96}=9.214, P<0.0001\right)$. Anemones containing the homologous $S$. minutum showed significantly lower levels of caspase-like activity than the heterologous $S$. trenchii and $S$. voratum, with an average activity at day 28 of $2.808 \pm 0.731 \mu \mathrm{mol}$ pNA $\min ^{-1} \cdot \mathrm{mg}$ host protein $^{-1}$. This lower caspase activity of $S$. minutum was similar to that of anemones containing the heterologous $S$. microadriaticum (Tukey post hoc, $P>0.05$ ) which had an average of $3.155 \pm 0.720 \mu \mathrm{mol} \mathrm{pNA} \min ^{-1} \cdot \mathrm{mg}$ host protein ${ }^{-1}, 28$ days after inoculation. In contrast, anemones containing the heterologous S. trenchii or S. voratum showed significantly higher caspase activity than the other species from day 14 onward (Tukey post hoc, $P<$ 0.05). At day 28 anemones inoculated with S. trenchii or S. voratum showed an average of $6.602 \pm 1.568$ and $7.850 \pm 1.215 \mu \mathrm{mol}$ pNA $\min ^{-1} \bullet \mathrm{mg}$ host protein ${ }^{-1}$ respectively.

Caspase activity ( $\mu$ mol pNA $\min ^{-1} \cdot \mathrm{mg}$ host protein $^{-1}$ ) was also significantly between different days (Fig. 4.3A; two-way ANOVA, F19, 58 = 41.506, $\mathrm{P}<0.0001$ ). All inoculated anemones showed high caspase activity one day after inoculation, while non-symbiotic anemones showed lower caspase activity. This followed by a decrease in caspase activity at day seven in all anemones, regardless of symbiont type.

These results corresponded with colonisation success, with the homologous S. minutum proliferating most rapidly, $S$. trenchii much slower, and $S$. voratum not persisting beyond day 7 (Fig. 4.2). Aposymbiotic anemones showed low caspase levels throughout most of the experiment, however at day 28 they showed high caspase activity, with an average of $7.762 \pm$ $1.049 \mu \mathrm{mol}$ pNA $\min ^{-1} \bullet \mathrm{mg}$ host protein ${ }^{-1}$.

Confocal visualization of caspase activity showed the same response as the assay, with anemones inoculated with either $S$. minutum or $S$. microadriaticum showing less caspase activity than anemones inoculated with $S$. trenchii or S. voratum (Fig. 4.3B).

\subsubsection{Correlation between caspase activity and algal density}


There was no significant correlation between caspase activity and algal density for each symbiont species when data for all time-points was combined (Fig. 4.4A-D; Pearson correlation, $P>0.05$ for all treatments). Similarly, there was no correlation between caspase activity and algal density for any of the symbiont species when each time-point was analysed separately (Pearson correlation, $P>0.05$ for all treatments and time-points), except for $S$. minutum and $S$. microadriaticum at day 28 , when a negative correlation was found between algal density and caspase activity.

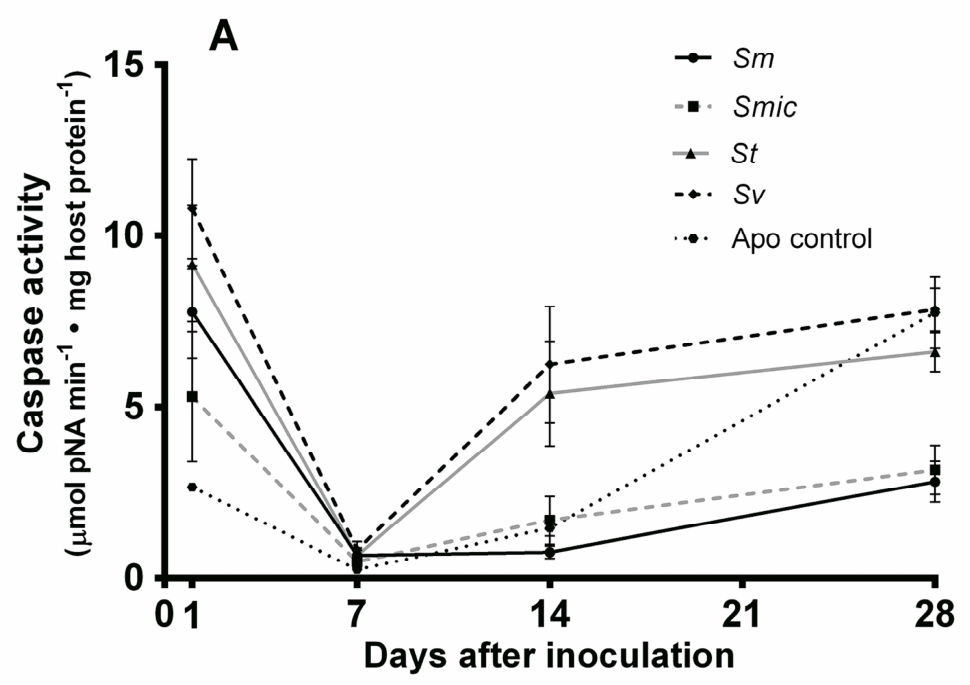

B

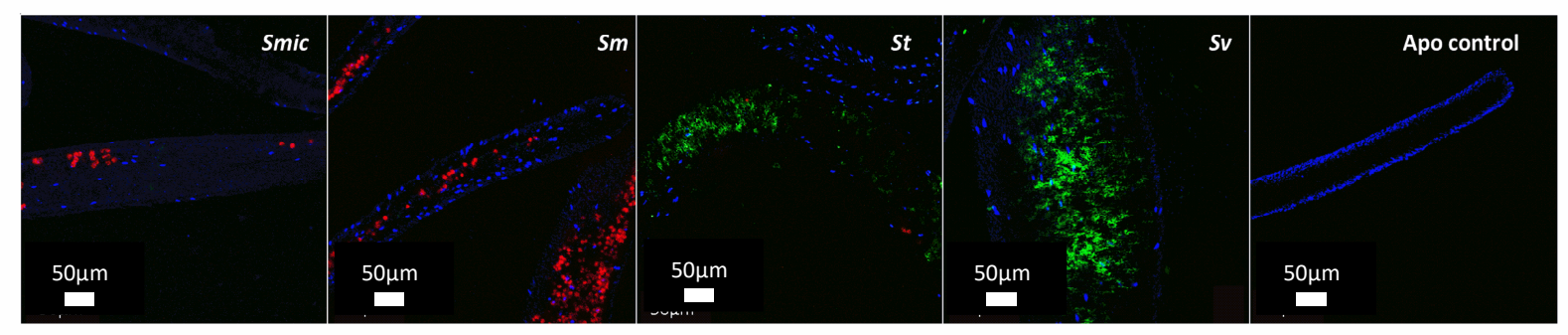

Fig. 4.3. Caspase activity differed with symbiont type. (A) Caspase-like enzyme activity of Aiptasia ( $\mu$ mol pNA $\min ^{-1} \bullet \mathrm{mg}$ host protein ${ }^{-1}$ ) was quantified for 28 days after inoculation ( $\mathrm{n}$ $=7$ per each treatment, two-way ANOVA, Tukey post hoc). Points are means \pm SE. (B) Confocal visualisation of caspase activity (green) and symbiont colonisation success (red) in Aiptasia host (blue), when colonised by different Symbiodinium types. All images were taken 14 days after inoculation. $S m=$ Symbiodinium minutum; $S t=S$. trenchii; Smic $=S$. microadriaticum; $S v=S$. voratum. 

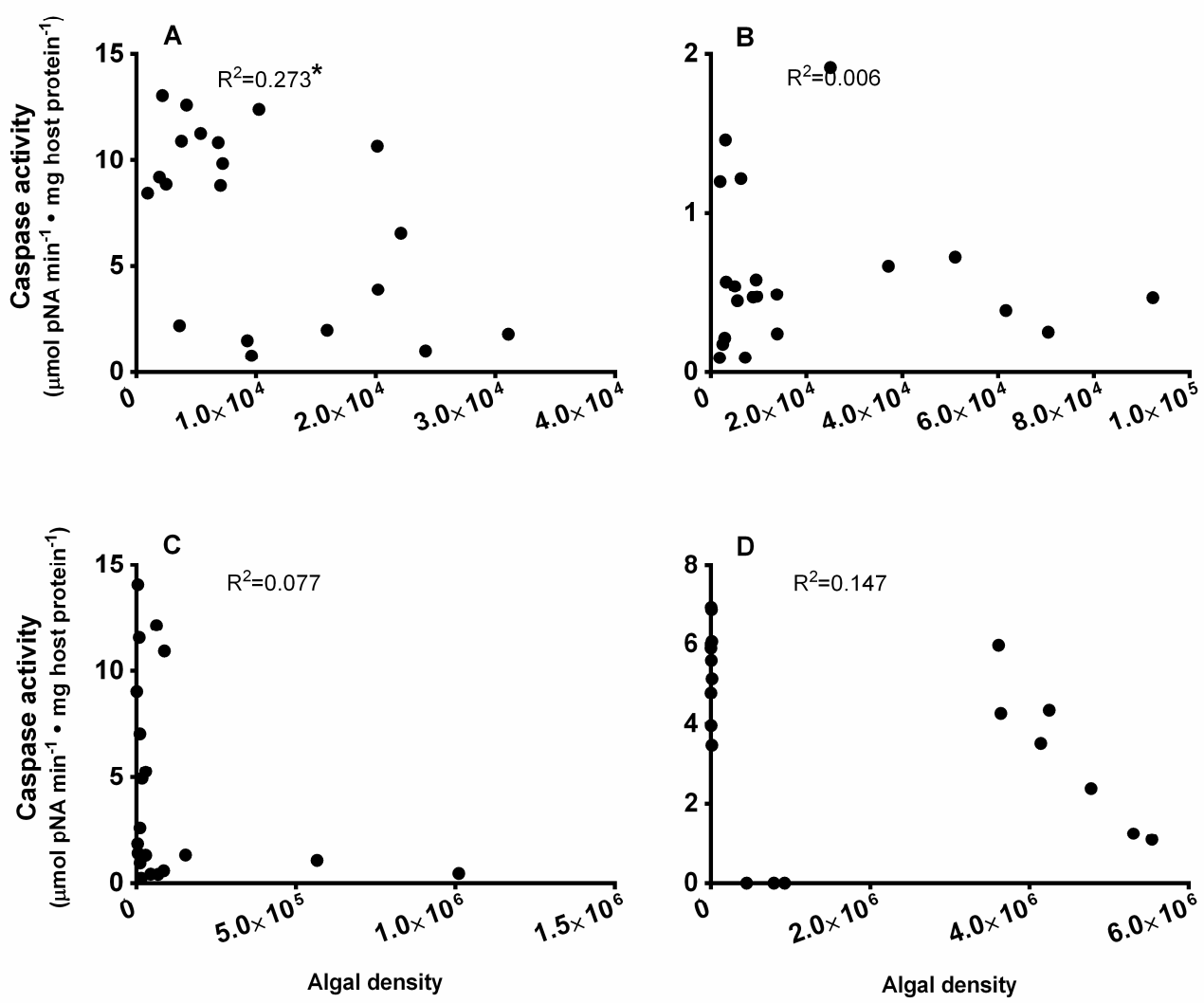

Fig. 4.4. Symbiont density has no effect on caspase activity in Aiptasia. Correlation between caspase-like enzyme activity $\left(\mu \mathrm{mol} \mathrm{pNA} \min ^{-1} \cdot \mathrm{mg}\right.$ host protein $\left.{ }^{-1}\right)$ and symbiont density (symbiont cells $\bullet \mathrm{mg}$ host protein $^{-1}$ ) over a period of 28 days after inoculation $(\mathrm{n}=28$ per algae type, Pearson correlation, $P>0.05$ for all comparisons). Points are means \pm SE. (A) Day 1; (B) day 7; (C) day 14; (D) day 28. The density of the symbiont species $S$. voratum was not detected after Day 7 and therefore was not analysed in this way.

\subsection{Discussion}

Apoptosis is known to play an important role in the control of symbiont proliferation in the cnidarian-dinoflagellate symbiosis (Dunn and Weis, 2009) and thermal bleaching (Pernice et al., 2011; Tchernov et al., 2011; Paxton et al., 2013). However, its role in determining the success of different symbiont types or species (homologous vs. heterologous) when colonising a particular host species, and hence in determining patterns of host-symbiont specificity, is less well explored. Here I show that symbiont species (independent of symbiont 
density) affects the activity of caspase-3 - a key apoptotic enzyme - in a manner that is consistent with colonisation success of the model sea anemone Aiptasia. More specifically, anemones inoculated with the homologous S. minutum and heterologous S. microadriaticum, exhibited less caspase activity than anemones harbouring the heterologous $S$. trenchii and $S$. voratum, both of which struggled to form a symbiosis with Aiptasia. Of note, S. trenchii was much slower to form a symbiosis here than it had been in my previous study (Chapter 2); the reason for this is unknown, but could reflect a change in the $S$. trenchii culture across time, either with respect to the algae themselves or the associated (and undefined) bacterial community.

\subsubsection{Temporal pattern of apoptosis during host colonisation}

Caspase activity changed significantly across time, as the host was colonised by its symbionts. Irrespective of symbiont type, all anemones showed the same general pattern, with induction of caspase-3 activity upon inoculation, followed by a marked decline in activity over the subsequent week, and then an increase (either moderate or marked depending upon symbiont identity) across the remainder of the colonisation period measured.

My results are consistent with the several studies that have shown that the initial uptake of Symbiodinium and other phototrophic symbionts (e.g. Chlorella) by cnidarians is not necessarily host-symbiont specific (Jolley and Smith, 1980; McAuley and Smith, 1982; Fitt, 1984; Davy et al., 1997b). For example, Colley and Trench (1983) investigated the role of cell surface interactions in host-symbiont recognition in the jellyfish Cassiopeia xamachana. They showed that different types of freshly isolated algae were able to infect the jellyfish, however only a few types were able to persist and form a stable symbiosis, indicating that initial symbiont uptake does not necessarily predict the fate of the symbiont inside the host. Beyond this initial phase, however, the increasing activity of caspase- 3 after Day 7 is consistent with a subsequent 'sorting' phase (Jolley and Smith, 1980; McAuley and Smith, 1982), where the host selects between compatible and less or non-compatible symbionts, and controls the proliferation of even compatible symbionts. 
Between the first uptake phase and the sorting phase, caspase activity and hence apoptosis appeared to be massively downregulated, irrespective of symbiont identity. The reason for this is unknown, but it could relate to a 'calming down' of the host immune response after the initial challenge with the different symbionts, and before proliferation of the symbiont population necessitates a further increase in apoptosis as the host begins to exert control. It has been reported that coral larvae (Acropora palmata and Montastraea faveolata) show a similar gene expression profile in the early stages of colonisation, regardless of symbiont compatibility (Voolstra et al., 2009). In the presence of all symbiont species, heme-binding protein 2 (AOKF1561; plays a role in apoptosis) and 6-4 pho-tolyase (AOKF2211; involved in DNA repair) were the most highly downregulated genes in the early stages of colonisation, and sorting Nexin 7 (CAOG407; functions in cell communication) was the most highly upregulated gene. After several days though, coral larvae inoculated with compatible and incompatible strains of Symbiodinium behaved very differently, with a gene that triggers apoptosis (AOSF1475: death-inducer obliterator 1) being among the upregulated genes in larvae inoculated with incompatible symbiont species (Voolstra et al., 2009).

One unexpected result was that aposymbiotic anemones showed elevated caspase activity towards the end of the experiment, akin to that seen in the anemones colonised by the least successful symbionts. The reason for this is unknown, but it seems unlikely to be an experimental artefact given the much lower caspase activity seen in anemones colonised by the homologous $S$. minutum for instance. One possibility is therefore that the aposymbiotic state may induce a state of stress over time, though this requires further investigation.

\subsubsection{Differential induction of apoptosis and its role in host-symbiont specificity}

My results therefore suggest that host cell apoptosis plays an important role in the postphagocytosis 'winnowing' process that removes inappropriate symbionts from the host, expanding upon the findings of Dunn and Weis (2009). These authors showed differences in caspase activity that corresponded with colonisation success during the onset of symbiosis in larvae of the mushroom coral Fungia scutaria, where larvae inoculated with the homologous 
symbiont exhibited significantly less caspase activity than larvae inoculated with a heterologous symbiont. Such a process therefore seems likely to play an important role in the determination of host-symbiont specificity in the cnidarian-dinoflagellate symbiosis, for which there is good evidence. For example, while some hosts may contain a variety of symbiont types in early ontogeny, they may contain only one or a few types as adults (Coffroth et al., 2001; Belda-Baillie et al., 2002; Thornhill et al., 2006). Furthermore, while more recent evidence suggests that some corals can associate with a wider range of symbionts than initially thought, many of these additional symbiont types are at cryptic levels (Silverstein et al., 2012).

However, my results also indicate that symbiont proliferation inside the host is not solely related to apoptosis. In particular, S. microadriaticum is less productive (Chapter 2) and achieves a lower population density in Aiptasia (this study) than does the homologous $S$. minutum; it also translocates relatively little fixed carbon to its host in some other symbioses (Mieog et al., 2009). Despite this, caspase-3 activity in anemones colonised by S. microadriaticum mirrored that seen in anemones colonised by $S$. minutum throughout the experiment. The reason for this discrepancy warrants further investigation, but one possibility is that $S$. microadriaticum more successfully evaded the host's immune response than did the other two heterologous symbiont species, as seen in various pathogens and parasites during host invasion (Sacks and Sher, 2002; Cossart and Sansonetti, 2004; Gruenberg and van der Goot, 2006). Alternatively, S. microadriaticum may have limited its removal by more successfully controlling host anti-apoptotic signalling mechanisms (James and Green, 2004; Koul et al., 2004). If increased apoptosis does not explain the lower rate and extent of proliferation by $S$. microadriaticum in Aiptasia, then another selective mechanism(s) must be involved. Possibilities include expulsion of the algae (Stimson and Kinzie, 1991; McCloskey et al., 1996) and control of the symbiont cell cycle by the host (Smith and Muscatine, 1999; Dimond and Carrington, 2008).

In summary, I demonstrate an important role for host cell apoptosis in determining the differential success of different symbiont types in the cnidarian-dinoflagellate symbiosis and observed patterns of host-symbiont specificity. However, other cellular mechanisms are also likely involved. To understand how specificity arises in the cnidarian-dinoflagellate symbiosis, as well as what might constrain the evolution of novel host-symbiont pairings, for example in response to changing climate, further work is needed. 


\section{Chapter 5}

\section{Symbiont cell cycle progression is unaffected by symbiont identity}

\section{in a model cnidarian-dinoflagellate symbiosis}

\subsection{Introduction}

The ecological and evolutionary success of coral reefs is primarily a consequence of the mutualistic association between the coral host and dinoflagellates of the genus Symbiodinium (Muscatine et al., 2005). The dinoflagellates live inside the gastrodermal cells of the host cnidarian, providing it with photosynthetically-fixed carbon that supports host respiration, growth and reproduction (Muscatine, 1990). Typically, corals contain more than a million symbionts per $\mathrm{cm}^{2}$ of their colony surface (Falkowski et al., 1993; Smith and Muscatine, 1999), yet there is a finite capacity for harbouring symbionts that is dictated by the intracellular space available (Jones and Yellowlees, 1997; Davy et al., 2012). The regulation and coordination of both host and symbiont cell proliferation is therefore important for maintaining a stable association (Reimer, 1971; Falkowski et al., 1993; Wang et al., 2008; Davy et al., 2012). Indeed, failure to control this homeostasis may result in overgrowth of the host cells by the algal symbionts and associated host stress (Muscatine and Neckelmann, 1983), or alternatively a low symbiont density that may not be able to adequately support the host's metabolic demands (Muscatine and Neckelmann, 1983; Taylor et al., 1989).

One key way in which this state of 'dynamic equilibrium' may be maintained is through the regulation of symbiont cell growth by the host. The photosynthetic rate of the symbionts in the host is similar to that of free-living cells, suggesting that their growth rates might be similar (Chang et al., 1983), however cellular growth in the host is much slower (Falkowski et al., 1993). More specifically, flow cytometric analysis of Symbiodinium has revealed that the $G_{1}$ phase of its cell cycle is significantly longer in the symbiotic state than in the freeliving state, while far fewer symbionts are observed in a state of division in symbiosis than 
when in culture (Wilkerson et al., 1983; Hoegh-Guldberg and Smith, 1989b; Fitt et al., 1993; Jones and Yellowlees, 1997; Smith and Muscatine, 1999; Dimond and Carrington, 2008). It therefore appears that progression through the symbiont cell cycle is limited in hospite. The exact mechanism for this is unclear, though the availability of inorganic nutrients and/or other metabolites (Smith and Muscatine 1999), as well as the light/dark regime (Fitt, 2000), may play a role. Furthermore, it is unclear to what extent symbiont growth is limited by densitydependent resource limitation in the host's tissues versus active control by the host.

The genus Symbiodinium is genetically and physiologically diverse (Fitt et al., 1981; IglesiasPrieto et al., 2004; Robison and Warner, 2006; Hennige et al., 2009; Pochon and Gates, 2010), however host-symbiont associations typically exhibit some degree of specificity and therefore are non-random (Santos et al., 2004; Thornhill et al., 2006; LaJeunesse et al., 2008). The specificity arises through cellular, physiological and/or environmental interactions. For example, glycan-lectin interactions between the host and symbiont cells enable cell-cell surface recognition (Wood-Charlson et al., 2006; Logan et al., 2010b), while favourable host-symbiont nutritional interactions and downregulation of host immune responses can facilitate symbiosis establishment (Matthews et al., 2017). Once established, a greater nutritional potential, and a faster host growth and reproductive rate may further facilitate the survival and persistence of specific host-symbiont pairings (Starzak et al., 2014; Chapter 2). However, exactly how and to what extent different symbiont populations are regulated by a host at the cellular level is not well understood, though differential rates of apoptosis are thought to play a part (Dunn and Weis, 2009; Chapter 4). A further possibility is that progression through the symbiont cell cycle may be impacted to different degrees, with, for example, faster colonisation by homologous symbionts being related to faster progression through the cell cycle, and hence more rapid cell division and proliferation through the host's tissues.

Here, I therefore compared cell cycle progression between different homologous and heterologous symbionts when colonising the same host species, with measurements taken across time until the host was fully populated. I challenged the sea anemone Exaiptasia pallida (Grajales and Rodríguez, 2014; commonly referred to as 'Aiptasia'), a widely-adopted model system for the study of cnidarian-dinoflagellate symbiosis (Weis et al., 2008; Baumgarten et al., 2015), with three different Symbiodinium species. These were the 
homologous species Symbiodinium minutum (ITS2 type B1; Thornhill et al., 2013), and the heterologous S. microadriaticum and S. trenchii (types A1 and D1a, respectively; Schoenberg and Trench, 1980c; Pettay and LaJeunesse, 2009). I hypothesized that heterologous symbiont types would show slower progression through the cell cycle, while the homologous symbiont type would exhibit faster progression through the cell cycle, consistent with its more rapid colonisation of the host.

\subsection{Materials and Methods}

\subsubsection{Experimental organisms}

A clonal culture of the symbiotic sea anemone Aiptasia (culture ID: H2), originally from Hawaii), was grown in the lab at $25^{\circ} \mathrm{C}$ and an irradiance of $40 \mu \mathrm{mol}$ photons $\bullet \mathrm{m}^{-2} \cdot \mathrm{s}^{-1}$ (12-h light/12-h dark), and fed twice weekly with freshly hatched Artemia nauplii. Aposymbiotic (i.e. symbiont-free) anemones were generated by incubating anemones in a solution of 0.27 $\mathrm{mM}$ menthol/AFSW $(0.22 \mu \mathrm{m}$-artificial filtered seawater, Instant Ocean, VA, USA; $32 \mathrm{ppt})$ for $8 \mathrm{~h}$, after which the menthol/AFSW was removed and the anemones incubated in $5 \mu \mathrm{M}$ DCMU/AFSW (3-(3,4-dichlorophenyl)-1,1-dimethylurea) overnight, as described by Matthews et al. (2015), with the treatment being repeated daily for four weeks or until no symbionts were present, as determined by fluorescence microscopy (AXIO, Zeiss USA; 635 $\mathrm{nm}$ laser, 655-755 $\mathrm{nm}$ emission filter).

Three species of cultured symbiotic dinoflagellates were chosen as inoculates (see Chapter 2, Table 2.1 for details). The algae were sub-cultured from long-term (> 5 years) laboratory stocks and grown in silica-free $\mathrm{f} / 2$ medium (National Center for Marine Algae and Microbiota, ME, USA) at $25{ }^{\circ} \mathrm{C}$ and an irradiance of $40 \mu \mathrm{mol}$ photons $\bullet \mathrm{m}^{-2} \cdot \mathrm{s}^{-1}$, on a $12-\mathrm{h}$ light/12-h dark regime (ViparSpectra-Timer 165W). All cultures were sampled for experimental use during the log phase of growth. 


\subsubsection{Inoculation of aposymbiotic sea anemones with symbionts}

Following one week of starvation, anemones of similar size $(2-3 \mathrm{~mm}$ oral disc diameter; $\mathrm{n}=$ 384 anemones) were transferred to clear plastic 12-well plates (one anemone per well) filled with $3 \mathrm{ml} 0.2 \mu \mathrm{m}$ AFSW and allowed to settle for one day. Each Symbiodinium culture was diluted in $25 \mathrm{ml} \mathrm{AFSW}$, and one drop of Artemia sp. nauplii suspension added to give the same final concentration of $\sim 7 \times 10^{5}$ cells $/ \mathrm{ml}$ for each Symbiodinium culture. The anemones were inoculated by pipetting $1 \mathrm{ml}$ of this dinoflagellate suspension onto the oral disc of each anemone with a glass pipette. The symbiont cell cycle was then monitored over a period of six weeks, with sampling at 1, 2, 4 and 6 weeks post-inoculation. During this time, the anemones were fed twice weekly with Artemia nauplii and the AFSW was changed every other day. Seawater temperature was maintained at $25^{\circ} \mathrm{C}$ in a controlled incubator, and the irradiance was held at $\sim 40 \mu \mathrm{mol}$ photons $\bullet \mathrm{m}^{-2} \cdot \mathrm{s}^{-1}$ on a 12-h light/12-h dark cycle (LEDENET 24V cold white 6500K-7000K LED strip, Enet Light Technology Co., Limited, China)

\subsubsection{Sample preparation}

At each time point, anemones were sampled in the morning between 8 and 10 am, 1-3 hours after the light period started, using the following method. Four randomly chosen anemones per treatment were grouped together to form one sample $(n=8$ samples per treatment at each time point), rinsed with AFSW and homogenized (Tissue terror-985370, Biospec products) in $1 \mathrm{ml}$ 1x phosphate-buffered saline (PBS; $\mathrm{pH} 7.4$ ). The homogenate was centrifuged (CS-15R, Beckman Coulter ${ }^{\mathrm{TM}}$ ) for $5 \mathrm{~min}$ at $500 \times \mathrm{g}$ at $4{ }^{\circ} \mathrm{C}$, to separate algal cells from anemone tissue, and the supernatant was discarded. The algal pellet was washed two more times with PBS and centrifuged as described above. The algal pellet was then fixed with $70 \%$ ice-cold ethanol and the algae were placed under bright light $\left(\sim 250 \mu \mathrm{mol}\right.$ photons $\bullet \mathrm{m}^{-2} \cdot \mathrm{s}^{-1}$; LEDENET 24V cold white 6500K-7000K LED strip, Enet Light Technology Co., Limited, China) for an hour at room temperature to bleach the chlorophyll. The samples were then centrifuged for $10 \mathrm{~min}$ at $500 \mathrm{x}$ g at $4{ }^{\circ} \mathrm{C}$ to separate the algae from the ethanol, and the algae resuspended in $30 \mu \mathrm{g} / \mathrm{ml}$ propidium iodide (PI) in PBS with $0.1 \%$ Triton-X and $10 \mu \mathrm{g} / \mathrm{ml}$ RNase. The cells were left to stain overnight in the dark at $4{ }^{\circ} \mathrm{C}$. This dye is stoichiometric 
i.e. it binds in proportion to the amount of DNA present in the cell. In this way cells in $\mathrm{G} 2+\mathrm{M}$ phase (containing $2 \times$ DNA) will be approximately twice as bright as cells in G1 phase (containing $1 \times$ DNA)

\section{Cell cycle measurement and analysis}

The DNA content per sample was measured based on DNA-PI complex fluorescence at 488/606 nm $\left(E_{x} / E_{m}\right)$ using a CytoFlex $S$ flow cytometer equipped with CytoExpert version 2.0.0.153 (Beckman Coulter Inc., IN, USA). Forward scatter count (FSC; measures cell size) and side scatter count (SSC; measures cell complexity) were recorded with linear gain settings. Laser energy and gains were set manually, and the calibration settings were applied to all samples. A minimum of 10,000 events were recorded for each sample. Four control samples were used to define the gating window for FSC that was specific to the dinoflagellate symbionts: (1) unstained algal culture; (2) stained algal culture; (3) stained aposymbiotic anemone cells; and (4) stained symbiotic anemone cells (Fig. 5.1). Events recorded in the PI channel were restricted to those falling within the FSC gating window. The proportion of the cell population in $\mathrm{G}_{1}$ phase (containing $1 \times \mathrm{DNA}$ ), $\mathrm{G}_{2}+\mathrm{M}$ phase (containing $2 \times$ DNA) and $\mathrm{S}$ phase (intermediate DNA content) were determined graphically from the PI fluorescence using FlowJo 10 (Fig. 5.1).

To estimate colonisation success, the number of cells in the dinoflagellate gating window was divided by the total number of cells in the sample (i.e. the percentage of all cells identified as being algal). This acted as a proxy for symbiont density in the anemone's tissues. 
A

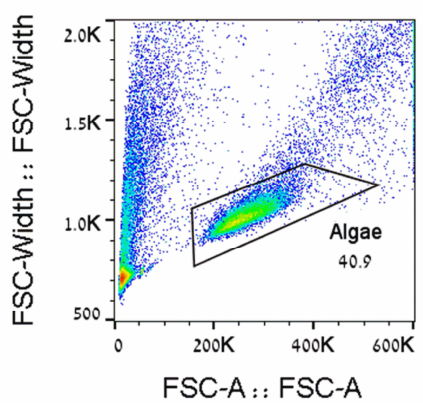

B

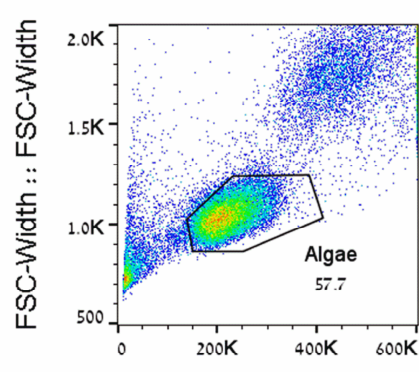

FSC-A : : FSC-A

C
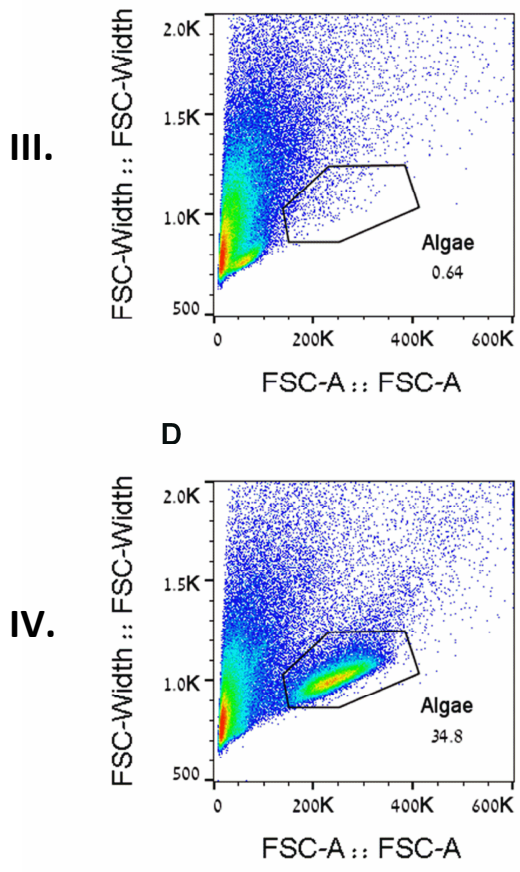

E

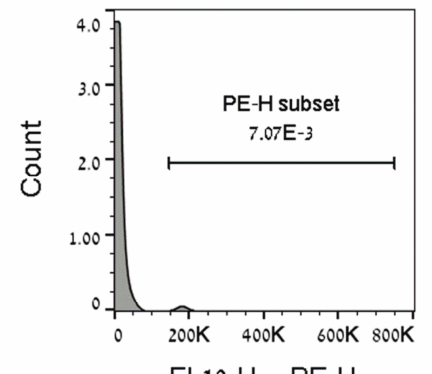

FL10-H : : PE-H

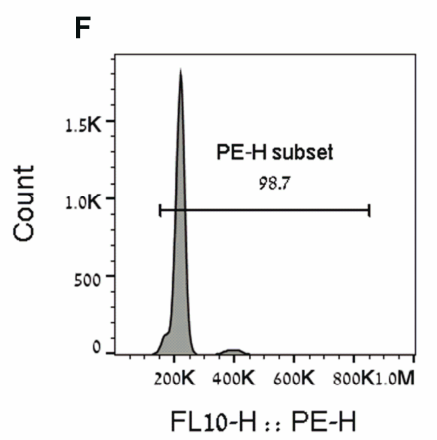

G

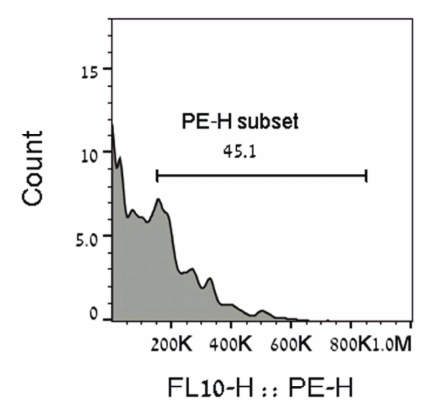

H

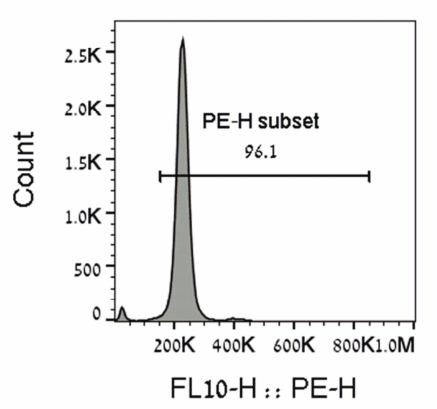

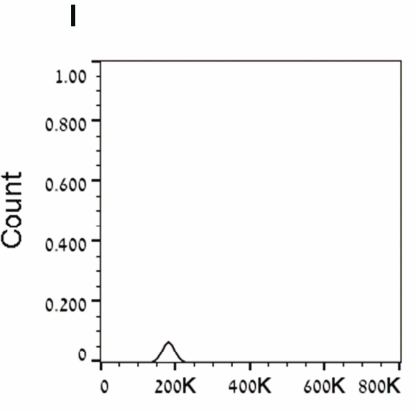

FL10-H : : PE-H

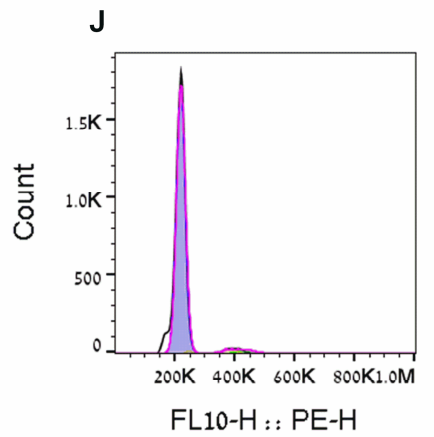

K

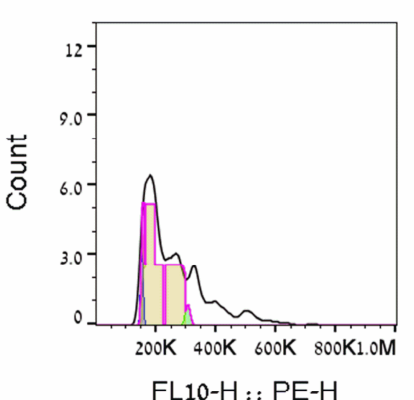

L

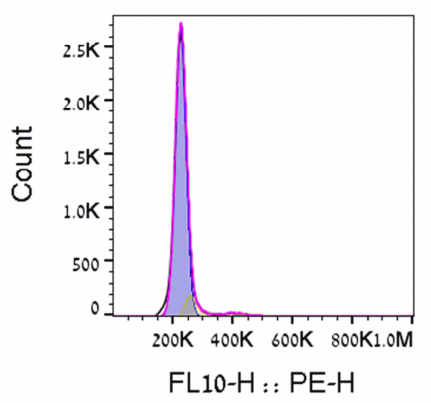

Fig. 5.1. Optimisation of the flow cytometry method. Four control samples were used to define the flow cytometry gating window specific to the dinoflagellate symbionts (rows): I. unstained cultured algae; II. propidium iodide (PI)-stained cultured algae; III. PI-stained aposymbiotic anemone cells; and IV. PI-stained symbiotic anemone cells. (A-D) Forward scatter count-area (FSC-A); (E-H) a subset of stained algae using the PE-H channel; (I-L) graphic cell cycle of the algae. Events recorded in the PE channel (E-L) were restricted to events falling within the FSC gating window. The proportion of the cell population in each cell cycle phase $\left(\mathrm{G}_{1}, \mathrm{~S}, \mathrm{G}_{2}+\mathrm{M}\right)$ was determined graphically from the PI fluorescence, using FlowJo 10. 


\subsubsection{DNA sequencing}

Symbiont type was verified for each culture. Symbiodinium samples in DMSO buffer (dimethyl sulfoxide) were re-suspended, and $10 \mu \mathrm{l}$ of each sample added to a new tube containing $50 \mathrm{mg}$ glass beads (BioSpec products) and placed in a TissueLyser (Sceintific Industries) bead beater for $1 \mathrm{~min}$ at $50 \mathrm{~Hz}$. Ninety microliters of $\mathrm{ddH}_{2} \mathrm{O}$ were added, and lysed cell material was pelleted at $16,100 \times \mathrm{g}$ for $10 \mathrm{~min}$ at $4{ }^{\circ} \mathrm{C}$. A $50 \mu$-aliquot of the resulting supernatant was transferred to a new tube containing $50 \mu \mathrm{l}$ cold molecular-grade isopropanol, and samples were re-pelleted as described above. Samples were washed twice in $200 \mu 170 \%$ ethanol, centrifuged and then dried at room temperature. Fifty microliters of $1 \mathrm{M}$ Tris ( $\mathrm{pH}$ 8) were added to each tube and samples were further extracted by bead beating at 30 $\mathrm{Hz}$ for $1 \mathrm{~min}$ and then stored at $-20^{\circ} \mathrm{C}$ until further analysis. Polymerase chain reaction (PCR) was performed using the following Symbiodinium-specific ITS2 (internal transcribed spacer region 2) primers: forward primer (ITS2infor) 5'-GAATTGCAGA ACTCCGTG-3'; reverse primer (ITS2rev) 5'-CCTCCGCTTACTTATATGCTT-3'. Reactions were performed using GoTaq $^{\mathrm{R}}$ (Promega, WI, USA) in a total volume of $25 \mu$ with an amplification profile consisting of: 1 cycle of 3 min $95{ }^{\circ} \mathrm{C}$; 40 cycles of $15 \mathrm{~s} 95^{\circ} \mathrm{C}, 15 \mathrm{~s} 56{ }^{\circ} \mathrm{C}$ and $10 \mathrm{~s} 72{ }^{\circ} \mathrm{C}$; and a final hold temperature of $4{ }^{\circ} \mathrm{C}$. PCR products were sequenced by the Center for Genome Research and Biocomputing (Oregon State University, OR, USA). Sequences were aligned using Geneious Pro 4.8.5 and a BLAST search was performed in the National Centre for Biotechnology Information (NCBI) database to identify the sequences. In all cases, the expected genotypes were present and multiple genotypes were never detected in any sample.

\subsubsection{Statistical analysis}

Each sample produced percentages of the three different cell cycle phases $\left(\mathrm{G}_{1}, \mathrm{~S}, \mathrm{G}_{2}+\mathrm{M}\right)$ as well as total cell counts that were analysed by the flow cytometer. The data were fitted to a multinomial logistic regression using the nnet package in $\mathrm{R}$. The fixed factors of time and symbiont species, and their interaction were included and tested against the null. The overall significance of each factor was established with a likelihood ratio test (LRT). The interaction term did not improve the model in all cases, so it was usually dropped. See Appendix 6 for more results. Results are expressed as mean \pm standard error (SE). 


\subsection{Results}

All three Symbiodinium species colonised Aiptasia, with no cross-contamination of symbiont species between treatments. The homologous S. minutum was the most successful species, achieving a maximum of $23.42 \pm 1.45 \%$ of the total measured cells four weeks after inoculation. This compared to the highest values of only $3.87 \pm 0.91 \%$ and $4.26 \pm 1.50 \%$ for the heterologous $S$. microadriaticum and S. trenchii at the same time point, respectively.

Cell cycle progression was not significantly different between Symbiodinium species (LRT, $\chi^{2}=0.378, \mathrm{df}=4, P=0.9842$; Fig. 5.2, 5.3, Appendix 6). While the percentage of cells in the different phases of the cell cycle did not change significantly in any of the different symbionts (LRT, $\chi^{2}=9.394$, $\mathrm{df}=6, P=0.1526$ ), a clear trend is apparent from visual inspection of Fig. 5.3. In particular, the total percentage (i.e. all symbiont species combined at each time point) of cells in the $\mathrm{G}_{1}$ phase declined by $29.66 \%$ two weeks after inoculation relative to week one, reaching its lowest level $(59.88 \pm 4.00 \%$ and $42.12 \pm 4.42 \%$ one and two weeks after inoculation, respectively). The total percentage of cells in the $\mathrm{G}_{1}$ phase then increased at weeks four and six, with an average of $73.04 \pm 0.83 \%$ and $76.71 \pm 1.88 \%$, respectively (Fig. 5.3). The total percentage of cells in the $\mathrm{G}_{2}+\mathrm{M}$ phase showed the opposite pattern, reaching its peak of $30.52 \pm 6.57 \%$ two weeks after inoculation before declining to only $1.33 \pm 0.28 \%$ by week 6 . The total percentage of cells in $\mathrm{S}$ phase decreased progressively at each sampling point, from $30.77 \pm 3.71 \%$ at week one to $20.53 \pm 1.88 \%$ cells at week six (Fig. 5.3). 

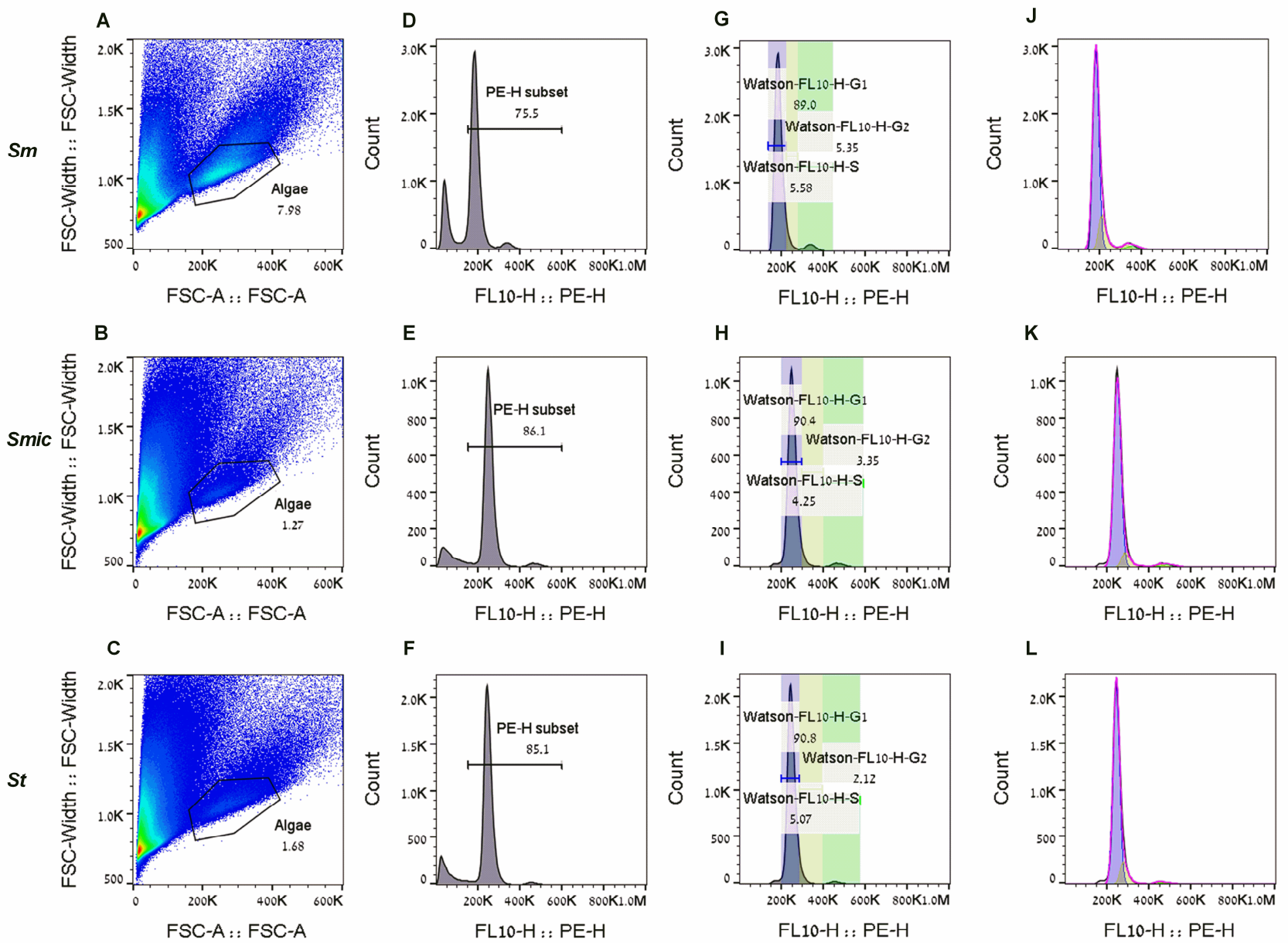

Fig. 5.2. Flow cytometry analysis of cell cycle progression by homologous and heterologous symbionts in Aiptasia. Cell cycle analysis of different symbiont species (rows; Symbiodinium minutum, S. microadriaticum, S. trenchii) six weeks after inoculation $(\mathrm{n}=4$ anemones per sample; 8 samples per treatment). (A-C) Forward scatter count-area (FSC-A); (D-F) a subset of stained algae using the PE-H channel; (G-I) percentage of cells in different cell cycle phases $\left(\mathrm{G}_{1}, \mathrm{~S}, \mathrm{G}_{2}+\mathrm{M}\right)$; (J-L) graphic showing cell cycle of the algae. The algal signal was gated based on the controls (Fig. 5.1). Events recorded in the PE channel (D-L) were restricted to events falling within the FSC gating window using FlowJo 10. $S m=S$. minutum, Smic $=S$. microadriaticum, $S t=S$. trenchii . 


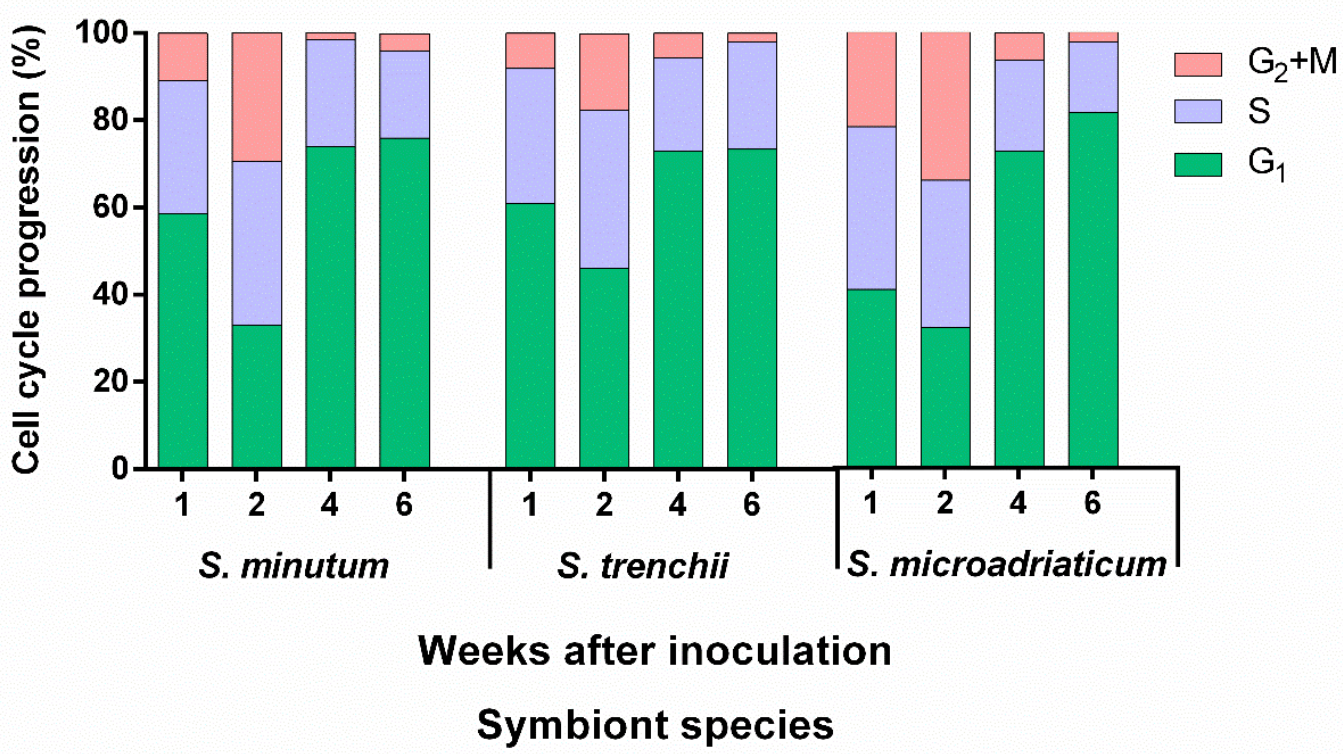

Fig. 5.3. Pattern of cell cycle progression was similar between different symbiont species. Cell cycle progression (\%) of each cell cycle phase $\left(\mathrm{G}_{1}, \mathrm{~S}, \mathrm{G}_{2}+\mathrm{M}\right)$ was quantified for six weeks after inoculation of Aiptasia with either Symbiodinium minutum, $S$. microadriaticum or $S$. trenchii $(\mathrm{n}=4$ anemones per sample; 8 samples per treatment per time point). The data for $S$. microadriaticum at week one were quantified from one observation only due to low symbiont densities.

\subsection{Discussion}

Symbiont cell cycle progression exhibited a similar pattern in Aiptasia, irrespective of symbiont identity. The first two weeks of host colonisation were characterized by the highest proportion of cells being in the $\mathrm{S}$ and $\mathrm{G}_{2}+\mathrm{M}$ phases, while in the subsequent weeks a greater proportion of cells were in the $\mathrm{G}_{1}$ phase. This pattern is consistent with rapid proliferation of the symbiont cells in the initial stages of colonisation, followed by reduced growth, as was reported in Chapter 2. Furthermore, previous studies involving the infection of aposymbiotic cnidarians, including Aiptasia, have shown that the symbiont growth rate may reach as much as $0.4 \mathrm{~d}^{-1}$, a similar rate to that seen in culture, before declining to a steady state of $<0.02 \mathrm{~d}^{-1}$ (Trench, 1971c; Schoenberg and Trench, 1980a; Berner et al., 1993). Similarly, when measured in terms of the mitotic index (i.e. the percentage of dividing cells), a value of just 
$1.80-2.69 \%$ has been reported in fully symbiotic Aiptasia, combined with a lengthy $\mathrm{G}_{1}$ phase (Smith and Muscatine, 1999).

The reduced cellular growth of the symbionts, and their more protracted $\mathrm{G}_{1}$ phase, in the later stages of host colonisation, suggest that their growth becomes limited (Jones and Yellowlees, 1997). What the exact mechanism is for this is still unclear, but possibilities include the production of an unidentified growth-inhibiting factor(s) (Muscatine and Pool, 1979; Douglas and Smith, 1984) or a "division factor" by the host that regulates the cell cycle (Muscatine and Pool, 1979; McAuley, 1982; McAuley, 1985a, b; McAuley and Muscatine, 1986), reduced light availability through self-shading by the symbionts (Crossland and Barnes, 1977), and/or the restriction of nutrients to the symbiont, for instance by host assimilation of ammonium via the action of glutamine synthetase or glutamate dehydrogenase (Cook and D’Elia, 1987; Cook et al., 1988; Miller and Yellowlees, 1989; Muscatine et al., 1989; HoeghGuldberg and Smith, 1989b; Falkowski et al., 1993; Pernice et al., 2012). Indeed, the link between nutrient supply and symbiont growth rate is well known, with the symbionts in wellfed anemones growing more than twice as fast as those in starved anemones (Smith and Muscatine, 1999). However, we are still a long way from understanding how symbiont cell growth is controlled and coordinated with that of the host, though 'omics methods, including transcriptomics, proteomics and metabolomics, have the potential to shed light on this, as they have in the legume-Rhizobium symbiosis where bacteria have been shown to undergo alteration in the transcriptional profiles of important cell cycle regulators in response to being in symbiosis (Perret et al., 2000; Mergaert et al., 2006; Jones et al., 2007; Oldroyd, 2013; Penterman et al., 2014).

While we do not exactly know how symbiont cell growth is regulated, it was nevertheless surprising that both the homologous and heterologous symbionts showed exactly the same pattern of cell cycle progression across time, especially given that $S$. minutum, $S$. microadriaticum and S. trenchii colonise Aiptasia at very different rates (Chapters 2, 3). Furthermore, we now know from a combined transcriptomic/metabolomic study that the physiological response to harbouring $S$. minutum or $S$. trenchii is very different, with $S$. trenchii inducing oxidative stress in the host's tissues and consequent upregulation in the host's immune pathways; this same symbiont also provides less nutritional benefit to Aiptasia than does S. minutum (Matthews et al., 2017). Moreover, when colonised by S. trenchii, the 
proteome of Aiptasia shows reduced expression of Niemann Pick proteins, which may be involved in sterol trafficking between the symbiotic partners, as well as glutamine synthetase, an important enzyme for the assimilation of inorganic nitrogen (Sproles et al., 2018). Taken together, these results suggest a less well integrated, functionally suboptimal symbiosis, with reduced capacity to regulate the symbiont population through the assimilation of ammonium for instance. Why this apparent physiological incompatibility has no effect on cell cycle progression requires further investigation, and a much more detailed, fundamental knowledge of exactly how symbiont growth is controlled in the cnidarian-dinoflagellate symbiosis.

Given that cell cycle progression showed no relationship to symbiont identity, despite the very different rates of host colonisation, other post-mitotic control mechanisms must be involved. Of particular note, previous work has identified host cell apoptosis as an important post-phagocytosis mechanism for sorting and controlling the symbiont population (Dunn and Weis, 2009). Indeed, in Aiptasia, host cell apoptosis is inversely related to colonisation success (Chapter 4). Other potential mechanisms include degradation of unwanted symbionts by the host cell (Titlyanov et al., 1996), expulsion of symbionts (Hoegh-Guldberg et al., 1987; Hoegh-Guldberg and Smith, 1989a; Stimson and Kinzie, 1991; McCloskey et al., 1996; Baghdasarian and Muscatine, 2000), and/or host autophagy (Dunn et al., 2007; Downs et al., 2009; Paxton et al., 2013). The relative importance of these various mechanisms in Aiptasia is not currently known, though in a recent study of the coral Stylophora pistillata, Lecointe et al. (2016) showed that symbiont cell division (measured as DNA synthesis) is >2fold higher than that of the host. These authors proposed that pre-mitotic and post-mitotic control must therefore be important for maintaining host-symbiont biomass, and showed that apoptosis becomes progressively more important as the coral progresses from the larval to adult stage. This topic warrants further detailed research in Aiptasia and other symbiotic cnidarians if we are truly to understand the ability of diverse symbiont types to establish new host-symbiont pairings in the long-term. 


\section{Chapter 6}

\section{General Discussion}

\subsection{Summary}

The capacity for corals to form novel partnerships with symbionts that may be better adapted to the new environmental regime (and vice versa) is an important consideration when assessing the ability for corals to adapt to climate change. However, relatively little attention has been given to the effects of hosting different symbiont types or species on holobiont physiology, the competitive interactions between these symbionts, or the capacity of the host to regulate populations of these different symbionts. Such factors likely play an important role in driving patterns of host-symbiont specificity and flexibility, and hence the potential for corals to respond to climate change (and indeed other aspects of environmental change) through the "shuffling" or "switching" of their symbionts. This thesis therefore examined the cellular and physiological events associated with hosting different symbiont types in the sea anemone Aiptasia, a model system for the cnidarian-dinoflagellate symbiosis, and how these events might contribute to observed patterns of host-symbiont specificity. I compared the cellular and physiological implications of hosting the homologous symbiont Symbiodinium minutum versus the heterologous symbionts $S$. microadriaticum, Symbiodinium C3, S. trenchii and S. voratum. Specifically, I tested:

5. The effect of symbiont identity on the rate and pattern of host tissue colonisation, and holobiont growth and reproduction;

6. The competitiveness of the homologous symbiont versus heterologous symbionts, under both control and elevated temperatures;

7. The ability of the host to regulate its symbiont population in response to homologous versus heterologous symbiont taxa via host-cell apoptosis; 
8. The ability of the host to regulate its symbiont population in response to homologous versus heterologous symbiont taxa via symbiont cell cycle regulation.

Overall I found that symbiont identity influenced the rate of symbiosis formation, physiological success of the holobiont, competitiveness of the symbiont, and the regulatory response of the host. Of particular interest, my results showed how the homologous symbiont proliferates faster, induces faster host growth and reproduction, and is more competitive even than heterologous symbionts with a reputation for being opportunistic and thermally resistant. This relative success was, in part, related to the lower incidence of apoptosis in those anemones colonised by the homologous symbiont versus the heterologous ones, whereas cell cycle progression of the symbiont played no obvious role in explaining the different colonisation patterns seen.

These findings raise some interesting questions about the relative importance of the different post-phagocytosis control mechanisms employed by the host to regulate its symbiont population, as well as the potential for the cnidarian-dinoflagellate symbiosis, and coral reefs more generally, to adapt to climate change. I will consider these questions in this final chapter, before highlighting priorities for future research.

\subsection{The relative importance of post-phagocytosis control mechanisms}

The cascade of molecular signalling referred to as 'the winnowing' is a necessary process in the establishment of a successful symbiosis and can be divided into pre- and post-phagocytic events. In this thesis, I explored the importance of two post-phagocytosis mechanisms - host cell apoptosis and symbiont cell cycle control. I found that, as algal density approached the level characteristic of a mature association, algal cellular growth rate declined as a result of a prolonged $G_{1}$ phase. However, cell cycle progression did not differ between different symbiont species, suggesting that the control of cell cycle progression might not be important for regulating the proliferation of incompatible or less beneficial symbionts. In contrast, my study showed that host cell apoptosis is an important mechanism by which the host eliminates incompatible symbionts and regulates symbiont proliferation. However, the situation is likely more complex than this, with a number of other post-phagocytosis mechanisms thought to be 
important, including symbiont expulsion (Hoegh-Guldberg et al., 1987; Hoegh-Guldberg and Smith, 1989a; Stimson and Kinzie, 1991; McCloskey et al., 1996) and degradation (Steele and Goreau, 1977; Titlyanov et al., 1996), and host cell autophagy (Dunn et al., 2007; Downs et al., 2009). When combined with host-symbiont recognition, and environmental pressures acting on the symbiosis, such mechanisms dictate the success of the symbiosis and patterns of host-symbiont specificity, as illustrated in Figure 6.1, which draws together the information generated in this thesis alongside existing knowledge of the symbiosis.

While each of these various mechanisms separately seems to be important for the control of the symbiont population, the relative importance of these mechanisms is still unclear. For example, while they were first thought to be unimportant (except under environmental stress; McAuley, 1981; Neckelmann et al., 1983; Douglas and Smith, 1984), expulsion and degradation are now thought to play an important role in the control of the symbiont population. A wide range of expulsion rates has been reported in the literature, from very low rates of expulsion ( 0.1 to $1.0 \%$ symbionts in a standing stock per day; Hoegh-Guldberg et al., 1987; Hoegh-Guldberg and Smith, 1989a; Stimson and Kinzie, 1991; Jones and Yellowlees, 1997) to very high ones, which sometimes can even exceed the daily growth rates of the population (McCloskey et al., 1996; Dimond and Carrington, 2008), suggesting that the importance of this regulatory mechanism varies between symbioses (Smith and Muscatine, 1999; Baghdasarian and Muscatine, 2000). We still know very little about the mechanisms underlying this symbiont expulsion and what triggers it, but further work on the mechanism of cellular phagocytosis will likely shed light on the mechanism of expulsion also. 


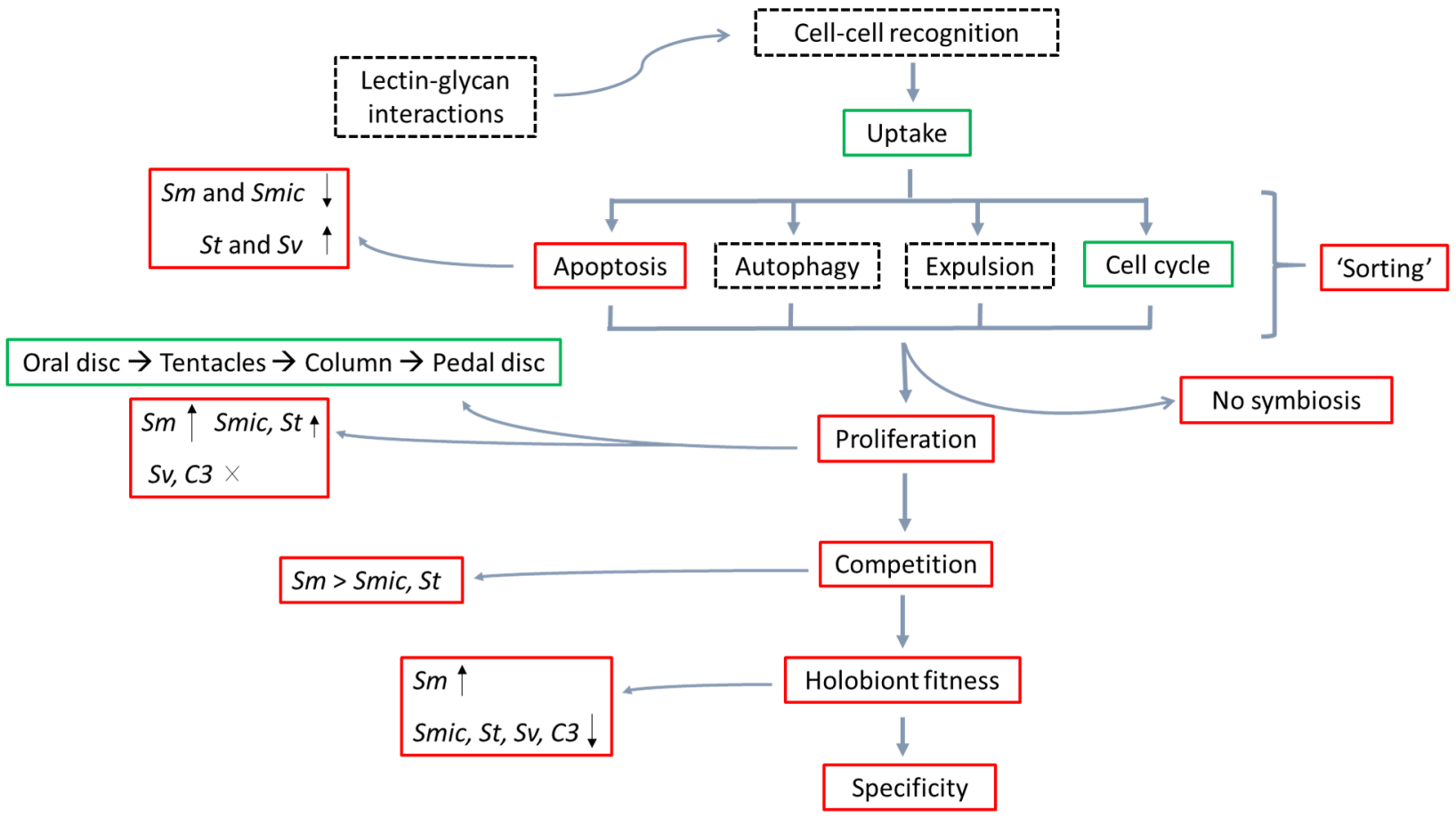

Fig. 6.1. The "winnowing" process: mechanisms of symbiont selection and regulation in the cnidarian-dinoflagellate symbiosis as highlighted by this thesis. Host mechanisms for controlling the symbiont population as well as eliminating incompatible symbionts, ultimately leading to a successful and potentially specific association. Complete squares are pathways studied in this thesis: red indicates mechanisms that are symbiont specific, while green indicates mechanisms that are not symbiont specific. Dashed squares indicate phenomena supported by the literature only, with no evidence currently as to whether they are symbiont specific or not. Sm = Symbiodinium minutum; $S t=$ S. trenchii; Smic $=$ S. microadriaticum $; \mathrm{C} 3=$ Symbiodinium phylotype C3; Sv $=$ S. voratum. One day post-inoculation, different symbionts were present at similar densities, suggesting that cell-cell recognition and uptake did not play a major part in the selection process (Chapters 2, 3, 4, 5). Once inoculated the symbionts are sorted by the host using several mechanisms. For example, apoptosis is a symbiont-specific mechanism, as the homologous species Sm and the heterologous species Smic induced significantly less caspase activity in the host than other heterologous symbionts (Chapter 4). In contrast, symbiont cell cycle regulation was not symbiont-specific, as all symbionts showed the same cell cycle pattern throughout the experiment (Chapter 5). Proliferation through the host's tissues was different between different symbiont taxa, with the homologous $\mathrm{Sm}$ being the fastest to proliferate (Chapter 2); however, the pattern of proliferation was similar between the different symbionts (Chapter 2). Sorting can also lead to little to no proliferation, as observed with $S v$ and $C 3$ (Chapter 2), with the symbiosis ultimately failing. The limited space inside the host's cells leads to competition, with the homologous $S m$ being the most competitive symbiont, even when competing with thermally robust symbionts (Smic and St) at elevated temperature (Chapter 3). Once the symbiosis is established, different symbiont taxa contribute differently to host fitness, as demonstrated by anemones colonised by the homologous symbiont being bigger and producing more pedal lacerates than anemones colonised by the various heterologous symbionts (Chapter 2). Ultimately, all of these mechanisms contribute to the specific symbiosis between Aiptasia and its homologous symbiont $S$. minutum in the Pacific region. 
Our knowledge of symbiont degradation is also somewhat limited, though possible mechanisms are symbiont apoptosis, maturation of the host cell symbiosome into a phagosome, and/or host cell autophagy (Davy et al., 2012). Given this range of regulatory mechanisms, the potential for a different relative importance in different symbioses (or in response to different symbionts) is therefore considerable. Currently, little is known about such differences, though a recent study by Lecointe et al. (2016) suggested that the relative importance of different pre-mitotic mechanisms might also differ with coral life-history stage. This study found that, in early development of Stylophora pistillata, apoptosis is not the main regulatory mechanism, however it becomes essential in the primary polyps and adult colonies of corals (Lecointe et al., 2016).

Once we better understand how different symbionts are regulated, and what ultimately dictates the competitiveness of these symbionts and the overall functional success of the symbiosis, we will be in a position to better understand the processes that underlie hostsymbiont specificity. We still have a long way to go in this regard, though the development of 'omics' methodologies is accelerating our rate of advancement. Notably, transcriptomics and metabolomics studies have recently revealed significant differences in gene expression and physiological processes in hosts associated with homologous vs. heterologous symbionts (Voolstra et al., 2009; DeSalvo et al., 2010; Mohamed et al., 2016; Matthews et al., 2017). For example, Matthews et al. (2017) showed that when Aiptasia is associated with the heterologous $S$. trenchii it exhibits an upregulation of G protein-associated stress signalling and lipid signalling pathways, which are active components in the stress response (see later for more discussion). However, we still have much to learn about the genes and pathways that govern host regulatory mechanisms and function in the cnidarian-dinoflagellate symbiosis. We should look to other areas of symbiology where the knowledge of symbiosis function is much more advanced, such as the agriculturally important Rhizobium-legume symbiosis. Here, for example, several studies have shown that the bacteria undergo alterations in the transcriptional profiles of important cell cycle regulators in favour of symbiosis, as is evident from comparative studies of Rhizobium versus other members of the

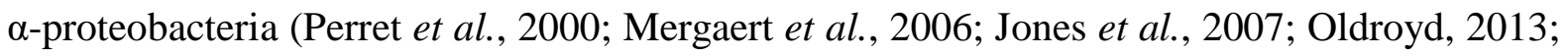
de Nisco et al., 2014; Penterman et al., 2014). Understanding the regulatory mechanisms and function of the cnidarian-dinoflagellate symbiosis is ultimately important for predicting the ability of coral reefs to withstand climate change - this is explored in the next section. 
The winnowing process has been studied in other symbiotic systems. By far, the two best studied systems in this regard are the Rhizobium-legume and Vibrio-squid symbioses. The bacterial symbiont $V$. fischeri colonizes the light-emitting organs of a number of marine squid and fish species (Lee and Ruby, 1994). The winnowing process leads to the selection of the specific symbiont by allowing $V$. fischeri to establish its dominance in the mucus over other microbes (Nyholm and McFall-Ngai, 2003). Once inside, V. fischeri induces a series of important developmental events in the host light organ (such as apoptosis of epithelial cells) that are thought to discourage colonization by other symbionts and promote colonization by V. fischeri (Nyholm et al., 2002; Kimbell and McFall-Ngai, 2004). The progression of colonization therefore occurs in a series of stages, with each step conferring greater specificity between the host and the symbiont. The symbiosis is maintained in dynamic equilibrium by a profound dial rhythm, mainly through expulsion of the symbiont population followed by regrowth to fill the crypts (Wier et al., 2010; McFall-Ngai et al., 2012). Two classes of $V$. fischeri occur in the natural environment of the symbiotic squid, which differ in their luminescence intensity (non-visibly luminous (NVL) and visibly luminous (VL)). Both classes are equally rapid and effective at colonizing the light organs of newly-hatched juvenile squid in laboratory conditions, however in the field only the NVL class is known to colonize the host (Lee and Ruby, 1994). Delayed competition experiments have shown that a light organ containing a VL strain and then inoculated with an NVL strain was replaced with the NVL strain within few days. Conversely, the reciprocal replacement of an NVL strain by a VL strain was never observed (Lee and Ruby, 1994; Sun et al., 2016). This pattern of events mirrors that reported in Chapter 3 for Aiptasia, suggesting that a strong link between the competitiveness of a symbiont and the extent of host-symbiont specificity might be a common feature.

In the Rhizobium-plant symbiosis, nitrogen-fixing rhizobial bacteria produce Nod factors that initiate a complex signal exchange response that is essential for bacterial invasion of the plant host (Oldroyd and Downie, 2004). Many of these downstream components of the Nod factor signal transduction have been identified (Cook, 2004; Oldroyd and Downie, 2004, 2006; Geurts et al., 2005). For example, the genes of the nodABC encode the proteins that are required for the structure of the core Nod factor (Perret et al., 2000) while other nod genes make modifications to Nod factors that impact host specificity. Defects in either plant or bacteria can cause a failure of symbiosome formation (Limpens et al., 2005; Combier et al., 
2006). The plant can also restrict the number of nodules on a colonized root; after an initial round of nodule formation additional nodulation events are subject to auto-inhibition (Van Brussel et al., 1992). Signalling, which is involved in defence signalling in plantmicroorganism interactions, is mediated by plant hormones (Sun et al., 2006). It seems that many signals for symbiosis and immune defence share similarities; for example NFR1-NFR5 are proteins needed for Nod factor recognition, yet overexpression of these proteins activates plant defence pathways and leads to cell death (Madsen et al., 2003).

The knowledge of the winnowing process and inter-partner communication in these two symbiotic systems is much more advanced than what is currently known about the cnidariandinoflagellate symbioses, even though our knowledge of these two well-studied symbioses is far from complete. My thesis goes some way to improving our understanding of the winnowing process in the cnidarian-dinoflagellate symbiosis, and in particular highlights a role of inter-symbiont competition and apoptosis, if not cell cycle regulation, but much more research is needed for us to understand how this symbiosis functions, and the commonalities between this and the Rhizobium-legume and squid-Vibrio associations, as well as other endosymbioses.

\subsection{Will corals be able to adapt to climate change?}

As seawater temperatures rise, there is a growing concern that cnidarians, such as corals, are approaching their maximum thermal limits (reviewed in Lesser, 2004). The possibility of algal community change inside the host (i.e. 'adaptive bleaching'; Buddemeier and Fautin, 1993 ) is therefore appealing and a subject of ongoing research (Goulet, 2006; Baker and Romanski, 2007; Oliver and Palumbi, 2011; Kemp et al., 2014; Núñez-Pons et al., 2017). Consistent with this mechanism, Thornhill et al. (2006) showed a community shift in the dominant symbiont species associated with adult colonies of the stony corals Montastrea annularis and Montastrea franksi while recovering from a bleaching event, with the thermally tolerant $S$. trenchii dominating during the stress but ultimately being replaced by other symbiont species that are naturally associated with these corals and are better able to support the host's nutritional demands (Thornhill et al., 2006). Such observations raise the possibility that thermally tolerant symbionts such as $S$. trenchii may offer a "nugget of hope" 
(Berkelmans and van Oppen, 2006) for reef corals. However, while it has been proposed that symbiont "shuffling" and perhaps even "switching" may occur in nature (Baker, 2003; Baker and Romanski, 2007; Silverstein et al., 2012), the situation is not necessarily that simple, as illustrated by the findings of this thesis. Indeed, my findings provide a mechanistic basis for why, while some corals contain multiple symbiont genotypes, others are very specific for just a single genotype from a particular clade (LaJeunesse et al., 2003; Goulet, 2006; Putnam et al., 2012; Poland and Coffroth, 2017). Indeed, my results suggest that the capacity for corals to adapt to climate change through a change in symbiotic partners, especially through switching, may be very limited.

Of particular interest, in Chapter 3 I show that Aiptasia from the Pacific, which is highly specific for $S$. minutum, struggles to form a symbiosis with other symbiont types (including the opportunistic $S$. trenchii) under elevated temperatures when $S$. minutum is still present, even though it has been suggested that $S$. minutum is thermally sensitive in culture (Suggett $e t$ al., 2008; Hawkins and Davy, 2012; but see Appendix 5). The continuing dominance of the homologous S. minutum emphasizes the importance of host-symbiont compatibility and suggests that Aiptasia is a highly specialist cnidarian that will retain its faithfulness for its homologous symbiont even as our climate warms. Indeed, my results show that Aiptasia would likely need to completely lose its homologous symbiont population for another symbiont type to stand any chance of achieving a dominant state.

While such a pattern of events is conceivable, my results in Chapter 2 suggest that the functionality of a novel symbiosis with Aiptasia may also be sub-optimal, with reduced productivity, growth and asexual reproduction. Given this, even if a new symbiosis should arise, its capacity to survive, compete and proliferate may be compromised in the wild. This observation is consistent with the recent findings of Matthews et al. (2017), who (as also mentioned above) showed that when Aiptasia is colonised by the heterologous symbiont $S$. trenchii, it undergoes oxidative stress and an upregulation of its immune system, as well as changes to its metabolite profile, consistent with the receipt of less photosynthetic carbon from the symbionts and a reduction in the availability of host energy reserves. Similarly, Starzak et al. (2014) found that $S$. voratum and type F5.1 induced high rates of host respiration that countered any photosynthetic benefits, and ultimately led to a low P:R ratio and little scope for host growth and reproduction. In these cases, however, there is a need to 
test these responses under a range of environmental scenarios, and across time in symbiosis, as host-symbiont interactions are not necessarily static. For example, when the symbiotic jellyfish Cassopeia xamachana acquires its symbiotic dinoflagellates via maternal inheritance rather than de novo with each population, the symbiosis exhibits a greater degree of mutualism (Sachs and Wilcox, 2006b). Similarly, in other microbial symbioses, symbiont selection can lead to a shift towards a more mutualistic state over time (Weeks et al., 2007; Bright and Bulgheresi, 2010).

Although these observations paint a somewhat gloomy picture, with limited potential for adaptation to climate change through partner change, it is important to note that the response to environmental change and the capacity to form new symbioses is likely species-specific (Lesser et al. 2013), with those hosts that naturally form symbioses with multiple symbiont types perhaps having a greater capacity to form novel associations, for instance. At the very least, such hosts may be able to "shift" their existing symbiont population to a dominant symbiont type that may be less beneficial but still better-than-nothing, and so confer a stronger chance of survival. However, in Chapter 2 I demonstrated that this may not be the case, with heterologous symbionts providing no discernible benefit in terms of host growth or asexual reproduction. This topic warrants further investigation to better predict the ability of corals to withstand environmental change, with a priority being extension of this work to actual corals from the model Aiptasia system.

Moreover, the general ecological assumption of endosymbiotic flexibility and stress susceptibility claims that flexibility enhances resilience in the cnidarian-dinoflagellate symbiosis (Buddemeier and Fautin, 1993). However, this assumption has been recently challenged, as generalist corals such as acroporids and pocilloporids are stress sensitive (Loya et al., 2001), while specialist corals that show high fidelity with respect to endosymbionts, such as poritids, are considered ecological 'winners' (Hoegh Guldberg and Salvat, 1995; Gates and Edmunds, 1999; Marshall and Baird, 2000; Woesik et al., 2011). Indeed, the relationship between a specialist cnidarian and its symbionts may be the outcome of stringent coevolution leading to a closely integrated and functionally optimal mutualism that can persist under both stable and stressful conditions. Therefore, highly specialised symbioses such as that between Aiptasia and S. minutum may be advantageous in the longrun rather than costly. 


\subsection{Future work}

The importance of a model system is well recognised across numerous taxa, such as bacteria, insects, plants and mammals (Davis, 2004), with Exaiptasia pallida ('Aiptasia') now being widely adopted in the field of cnidarian-dinoflagellate symbiosis (for more details see Chapter 1). However, there are some disadvantages in the use of this sea anemone, one of the most obvious of which is that it does not calcify and therefore has a different physiology with respect to carbon utilisation; it is also not a suitable model for the study of endoskeletal microbes (Tambutte et al., 1995; Enríquez et al., 2005; Wegley et al., 2007). Furthermore, in my thesis I found that Aiptasia displays a high degree of fidelity towards its homologous symbionts, even under thermal stress. Therefore, while it is an ideal model for the study of how such high levels of host-symbiont specificity arise, and the responses to foreign symbionts, it is less ideal for studies aimed at investigating symbiont community shifts or switches that may occur in more generalist corals such as Acropora and Pocillopora (Putnam et al., 2012). Such limitations highlight the urgent need to now extend the findings of this thesis to actual reef corals, to better inform our understanding of reef function of future predictions in the face of climate change. Furthermore, fundamental questions remain about aspects of the cnidarian-dinoflagellate symbiosis that would, in the first instance, still be best addressed with the Aiptasia model system. I therefore propose the following areas as priorities for future research.

First, I found a very distinctive spatial pattern of symbiont colonisation and symbiosis establishment that included a patchy pattern of symbiont proliferation in the host's tentacles (Chapter 2). This led me to hypothesise that the symbionts exit the host's cells, move through the gastrovascular cavity via ciliary water currents, and then invade other host cells elsewhere in the animal. Parrin et al. $(2012 ; 2016)$ observed within-colony symbiont migration in soft corals as a response to thermal stress, and suggested a similar mechanism of symbiont distribution to that hypothesized here. However, the exact mechanism by which this occurs has not been confirmed; similarly, the mechanism by which the symbionts grow and spread from one host cell to the next within a patch is completely unknown. Aiptasia remains an ideal system in which to further our understanding of these topics through detailed microscopical examination. 
Second, in Chapter 3 I report the highly specialist nature of the Aiptasia $-S$. minutum symbiosis from the Pacific region. However, this strong fidelity, including under elevated temperature, may not be typical in those hosts with a more flexible nature. It is therefore crucial to extend this type of work to other cnidarian hosts, and in particular corals, specifically comparing the competitiveness of symbionts inside a generalist versus a specialist host under thermal stress. Combined with this, it will be important to assess the physiological benefits of corals hosting different symbiont types under a range of thermal scenarios and across time, to determine the costs or benefits of establishing different symbioses at the level of the holobiont. Such information is hugely important to understanding the adaptive capacity of corals to climate change.

Finally, while I demonstrated a role for apoptosis but not cell cycle control in the regulation of less compatible symbionts and the establishment of host-symbiont specificity, we are still far from understanding the relative importance of different regulatory mechanisms. Indeed, we still lack a basic understanding of how even the regular symbiosis is maintained in a steady state, for example through the coordination of host and symbiont cell division. This is despite numerous attempts to explore different post-phagocytosis mechanisms for controlling the symbiont population (McCloskey et al., 1996; Titlyanov et al., 1996; Dimond and Carrington, 2008; Downs et al., 2009; Dunn and Weis, 2009). I therefore recommend further research on this subject, including: (1) elucidation of the molecules and signalling pathways such as those involved in host immune responses or nutritional exchange - that determine host-symbiont compatibility and hence the nature of the regulatory response employed; (2) characterisation of the symbiont and host cell cycles, through bioinformatics and experimental approaches; and (3) determination of the relative magnitude of the different regulatory mechanisms in both Aiptasia and reef corals, under a range of environmental scenarios. The fields of transcriptomics, proteomics and metabolomics offer new and exciting avenues for tackling these complex issues. 


\subsection{Conclusions}

This study aimed to investigate the physiological and cellular effects of hosting different symbiont phylotypes on the cnidarian-dinoflagellate symbiosis. As symbionts differ in their genotype as well as their physiological characteristics (such as thermal tolerance versus thermal sensitivity, or nutritionally beneficial versus less beneficial), I expected that different symbionts would be more advantageous to the host and hence more competitive under different environmental scenarios, and that the host's compatibility with its symbionts would be reflected in its regulatory response. My results were not always consistent with my hypotheses, with the homologous $S$. minutum being most beneficial and competitive, and establishing a symbiosis more readily with Aiptasia than several heterologous symbionts, which induced higher rates of apoptosis in the host than did the homologous symbiont. All of the symbionts exhibited the same patterns of host colonisation, however, and similar patterns of cell cycle progression. Most surprisingly, the competitiveness of $S$. minutum continued even at elevated temperature, when the likes of the thermally tolerant, opportunistic $S$. trenchii might have been expected to benefit at the expense of the homologous partner. Taken together, the findings of my thesis provide insight into how the highly specific Aiptasia $-S$. minutum symbiosis of the Pacific Ocean arises and is maintained, with implications for our understanding of similarly specific symbioses. Furthermore, my thesis sheds light on the potential to establish novel host-symbiont pairings and the adaptation of reef corals to climate change through changing partners. However, further work with corals themselves, including more generalist species, and under a range of environmental scenarios and timeframes, is needed to better predict the changes that coral reefs may undergo in the future as our climate changes. 


\section{References}

Alberts, B., D. Bray, K. Hopkin, A. Johnson, J. Lewis, M. Raff, K. Roberts and P. Walter. 2013. Molecular biology of the cell, Fourth Edition. pp. 865. Garland Science Press.

Alberts, B., A. Johnson, J. Lewis, M. Raff, K. Roberts and P. Walter. 2002. The cell cycle and programmed cell death. pp. 1463. Garland Science Press.

Badgley, B. D., F. Lipschultz and K. P. Sebens. 2006. Nitrate uptake by the reef coral Diploria strigosa: Effects of concentration, water flow, and irradiance. Mar. Biol. 149: 327-338.

Baghdasarian, G. and L. Muscatine. 2000. Preferential expulsion of dividing algal cells as a mechanism for regulating algal-cnidarian symbiosis. Biol. Bull. 199: 278-286.

Baker, A. C. 2001a. Ecosystems: Reef corals bleach to survive change. Nature 411: 765-766.

Baker, A. C. 2001b. Reef corals bleach to survive change. Nature 411: 765-766.

Baker, A. C. 2002. Is bleaching really adaptive? Reply to Hoegh-Guldberg et al. Nature 415: 602.

Baker, A. C. 2003. Flexibility and specificity in coral-algal symbiosis: diversity, ecology, and biogeography of Symbiodinium. Annu. Rev. Ecol. Evol. Syst. 34: 661-689.

Baker, A. C. and A. M. Romanski. 2007. Multiple symbiotic partnerships are common in scleractinian corals, but not in octocorals: Comment on Goulet (2006). Mar. Ecol. Prog. Ser. 335: $237-242$.

Banaszak, A., T. LaJeunesse and R. Trench. 2000. The synthesis of mycosporine-like amino acids (MAAs) by cultured, symbiotic dinoflagellates. J. Exp. Mar. ... 249: 219-233.

Barneah, O., V. M. Weis, S. Perez and Y. Benayahu. 2004. Diversity of dinoflagellate symbionts in Red Sea soft corals : mode of symbiont acquisition matters. Mar. Ecol. Prog. Ser. 275: 89-95.

Bary, A. De. 1879. Die erscheinung der symbiose. Verlag von Karl J. Trubn. Strassbg.

Baumgarten, S., O. Simakov, L. Y. Esherick, Y. J. Liew, E. M. Lehnert, C. T. Michell, Y. Li, E. A. Hambleton, A. Guse, M. E. Oates, , J. Gough, V. M. Weis, M. Aranda, J. R. Pringel, C. R. Voolstra. 2015. The genome of Aiptasia, a sea anemone model for coral symbiosis. Proc. Natl. Acad. Sci. 112: 11893-11898.

Belda-Baillie, C. a., B. K. Baillie and T. Maruyama. 2002. Specificity of a model cnidarian- 
dinoflagellate symbiosis. Biol. Bull. 202: 74-85.

Benayahu, Y. and M. H. Schleyer. 1998. Reproduction in Anthelia glauca (Octocorallia: Xeniidae). I. Gametogenesis and larval brooding. Mar. Biol. 131: 423-432.

Benayahu, Y., D. Weil and Z. Malik. 1992. Entry of algal symbionts into oocytes of the coral Litophyton arboreum. Tissue Cell 24: 473-482.

Beringer, J. E., N. Brewin, A. W. B. Johnston, H. M. Schulman and D. A. Hopwood. 1979. The rhizobium-legume symbiosis. Proc. R. Soc. B Biol. Sci. 204: 219-233.

Berkelmans, R. and M. J. . van Oppen. 2006. The role of zooxanthellae in the thermal tolerance of corals: a "nugget of hope" for coral reefs in an era of climate change. Proc. $R$. Soc. B Biol. Sci. 273: 2305-2312.

Berner, T., G. Baghdasarian and L. Muscatine. 1993. Repopulation of a sea anemone with symbiotic dinoflagellates: Analysis by in vivo fluorescence. J. Exp. Mar. Bio. Ecol. 170: $145-158$.

Biquand, E., N. Okubo, Y. Aihara, V. Rolland, D. C. Hayward, M. Hatta, J. Minagawa, T. Maruyama and S. Takahashi. 2017. Acceptable symbiont cell size differs among cnidarian species and may limit symbiont diversity. ISME J. doi:10.103.

Bongaerts, P., C. Riginos, T. Ridgway, E. M. Sampayo, M. J. H. van Oppen, N. Englebert, F. Vermeulen and O. Hoegh-Guldberg. 2010. Genetic divergence across habitats in the widespread coral Seriatopora hystrixand its associated Symbiodinium. PLoS One 5.

Bradford, M. M. 1976. A rapid and sensitive method for the quantitation of microgram quantities of protein utilizing the principle of protein-dye binding. Anal. Biochem. 72: 248254.

Bright, M. and S. Bulgheresi. 2010. A complex journey: Transmission of microbial symbionts. Nat. Rev. Microbiol. 8: 218-230.

Bronstein, J. L. 1994. Conditional outcomes in mutualistic interactions. Trends Ecol. Evol. 9: 214-217.

Buddemeier, R. W. and D. G. Fautin. 1993. Coral bleaching as an adaptive aechanism a testable hypothesis. Bioscience 43: 320-326.

Bull, J. 1994. Perspective: Virulence. Evolution (N. Y). 48: 1423-1437.

Burriesci, M. S., T. K. Raab and J. R. Pringle. 2012. Evidence that glucose is the major 
transferred metabolite in dinoflagellate-cnidarian symbiosis. J. Exp. Biol. 215: 3467-3477.

Cantin, N. E., M. J. H. van Oppen, B. L. Willis, J. C. Mieog and A. P. Negri. 2009. Juvenile corals can acquire more carbon from high-performance algal symbiosis. Coral Reefs 28: 405-414.

Cates, N. and J. J. A. McLaughlin. 1976. Differences of ammonia metabolism in symbiotic and aposymbiotic condylactus and cassiopea spp. J. Exp. Mar. Bio. Ecol. 21: 1-5.

Chang, S. S., B. B. Prézelin and R. K. Trench. 1983. Mechanisms of photoadaptation in three strains of the symbiotic dinoflagellate Symbiodinium microadriaticum. Mar. Biol. 76: 219229.

Chen, M.-C., Y.-M. Cheng, M.-C. Hong and L.-S. Fang. 2004. Molecular cloning of Rab5 (ApRab5) in Aiptasia pulchella and its retention in phagosomes harboring live zooxanthellae. Biochem. Biophys. Res. Commun. 324: 1024-33.

Chen, M.-C., Y.-M. Cheng, P.-J. Sung, C.-E. Kuo and L.-S. Fang. 2003. Molecular identification of Rab7 (ApRab7) in Aiptasia pulchella and its exclusion from phagosomes harboring zooxanthellae. Biochem. Biophys. Res. Commun. 308: 586-595.

Chen, M.-C., M.-C. Hong, Y.-S. Huang, M.-C. Liu, Y.-M. Cheng and L.-S. Fang. 2005. ApRab11, a cnidarian homologue of the recycling regulatory protein Rab11, is involved in the establishment and maintenance of the Aiptasia-Symbiodinium endosymbiosis. Biochem. Biophys. Res. Commun. 338: 1607-16.

Clayton, W. and H. Lasker. 1984. Host feeding regime andzooxanthellal photosynthesis in the anemone, Aiptasia pallida (verrill). Biol. Bull. 167: 590-600.

Coffroth, M. A., D. M. Poland, E. L. Petrou, D. a. Brazeau and J. C. Holmberg. 2010. Environmental symbiont acquisition may not be the solution to warming seas for reefbuilding corals. PLoS One 5: e13258.

Coffroth, M. A., S. R. Santos and T. L. Goulet. 2001. Early ontogenetic expression of specificity in a cnidarian-algal symbiosis. Mar. Ecol. Prog. Ser. 222: 85-96.

Coles, S. L. and B. E. Brown. 2003. Coral bleaching - capacity for acclimatization and adaptation. Adv. Mar. Biol. 46: 183-223.

Colley, N. J. and R. K. Trench. 1983. Selectivity in phagocytosis and persistence of symbiotic algae by the scyphistoma stage of the jellyfish Cassiopeia xamachana. Proc. R. Soc. London B Biol. Sci. 219: 61-82. 
Combier, J., F. Frugier, F. De Billy, A. Boualem, F. El-yahyaoui, S. Moreau, T. Vernié, T. Ott, P. Gamas, M. Crespi, et al. 2006. MtHAP2-1 is a key transcriptional regulator of symbiotic nodule development regulated by microRNA169 in Medicago truncatula. Genes and Development. 20: 3084-3088.

Cook, C. B. C., C. F. D'Elia and G. Muller-Parker. 1988. Host feeding and nutrient sufficiency for zooxanthellae in the sea anemone Aiptasia pallida. Mar. Biol. 98: 253-262.

Cook, C. B. and C. F. D'Elia. 1987. Are natural populations of zooxanthellae ever nutrientlimited? Symbiosis 4: 199-211.

Cook, C. B., G. Muller-Parker and C. D. Orlandini. 1994. Ammonium enhancement of dark carbon fixation and nitrogen limitation in zooxanthellae symbiotic with the reef corals Madracis mirabilis and Montastrea annularis. Mar. Biol. 118: 157-165.

Cook, D. R. 2004. Unraveling the mystery of Nod factor signaling by a genomic approach in Medicago trunactula. Proc. Natl. Acad. Sci. U. S. A. 101: 4339-4340

Cossart, P. and P. J. Sansonetti. 2004. Bacterial Invasion: The Paradigms of Enteroinvasive Pathogens. Science 304: 242-248.

Crossland, C. J. and D. J. Barnes. 1977. Gas-exchange studies with the staghorn coral Acropora acuminata and its zooxanthellae. Mar. Biol. 40: 185-194.

D'Elia, C. F. 1977. The uptake and release of dissolved phosphorus by reef corals. Limnol. Oceanogr. 22: 301-315.

D'Elia, C. F., S. L. Domotor and K. L. Webb. 1983. Nutrient uptake kinetics of freshly isolated zooxantellae. Mar. Biol. 167: 157-167.

D'Haeze, W. and M. Holsters. 2002. Nod factor structures, responses, and perception during initiation of nodule development. Glycobiology 12: 79-105.

Dahlmann, F., N. Biedenkopf, A. Babler, W. Jahnen-Dechent, C. B. Karsten, K. Gnirß, H. Schneider, F. Wrensch, C. A. O'Callaghan, S. Bertram, et al. 2015. Analysis of Ebola virus entry into macrophages. J. Infect. Dis. 212: S247-S257.

Dani, V., F. Priouzeau, M. Mertz, M. Mondin, S. Pagnotta, S. Lacas-Gervais, S. K. Davy and C. Sabourault. 2017. Expression patterns of sterol transporters NPC1 and NPC2 in the cnidarian-dinoflagellate symbiosis. Cell. Microbiol. 19: DOI: 10.1111/cmi.12753.

Davis, R. H. 2004. The age of model organisms. Nat. Rev. Genet. 5: 69-76. 
Davy, S. and C. Cook. 2001. The relationship between nutritional status and carbon flux in the zooxanthellate sea anemone Aiptasia pallida. Mar. Biol. 139: 999-1005.

Davy, S. K., D. Allemand and V. M. Weis. 2012. Cell biology of cnidarian-dinoflagellate symbiosis. Microbiol. Mol. Biol. Rev. 76: 229-261.

Davy, S. K., I. A. N. Lucas and J. R. Turner. 1996. Carbon budgets in temperate anthozoandinoflagellate symbioses. Mar. Biol. 126: 773-783.

Davy, S. K., I. A. N. Lucas and J. R. Turner. 1997b. Uptake and persistence of homologous and heterologous zooxanthellae in the temperate sea anemone Cereus pedunculatus (Pennant). Biol. Bull. 192: 208-216.

Davy, S. K., J. Turner and I. Lucas. 1997a. The nature of temperate anthozoan-dinoflagellate symbioses. Proc. 8th Int. Coral Reef Symp 2: 1307-1312.

Davy, S. K. and J. R. Turner. 2003. Early development and acquisition of zooxanthellae in the temperate symbiotic sea anemone Anthopleura ballii (Cocks). Biol. Bull. 205: 66-72.

Davy, S. K., K. J. T. Withers and R. Hinde. 2006. Effects of host nutritional status and seasonality on the nitrogen status of zooxanthellae in the temperate coral Plesiastrea versipora (Lamarck). J. Exp. Mar. Bio. Ecol. 335: 256-265.

de Nisco, N. J., R. P. Abo, C. M. Wu, J. Penterman and G. C. Walker. 2014. Global analysis of cell cycle gene expression of the legume symbiont Sinorhizobium meliloti. PNAS 111: 3217-3224.

DeSalvo, M. K., S. Sunagawa, P. L. Fisher, C. R. Voolstra, R. Iglesias-Prieto and M. Medina. 2010. Coral host transcriptomic states are correlated with Symbiodinium genotypes. Mol. Ecol. 19: 1174-1186. Blackwell Publishing Ltd.

Dimijian, G. G. 2000. Evolving together: the biology of symbiosis, part 1. Proc. (Bayl. Univ. Med. Cent). 13: 217-26.

Dimond, J. and E. Carrington. 2008. Symbiosis regulation in a facultatively symbiotic temperate coral: Zooxanthellae division and expulsion. Coral Reefs 27: 601-604.

Donner, S. D. 2009. Coping with commitment: Projected thermal stress on coral reefs under different future scenarios. PLoS One 4: e5712.

Donner, S. D., T. R. Knutson and M. Oppenheimer. 2007. Model-based assessment of the role of human-induced climate change in the 2005 Caribbean coral bleaching event. Proc. Natl. 
Acad. Sci. U. S. A. 104: 5483-5488.

Donner, S. D., W. J. Skirving, C. M. Little, M. Oppenheimer and O. Hoegh-Gulberg. 2005.

Global assessment of coral bleaching and required rates of adaptation under climate change. Glob. Chang. Biol. 11: 2251-2265.

Douglas, A. 1994. Symbiotic interactions. pp. 148. Oxford University Press, Oxford and New York.

Douglas, A. E. 2008. Conflict, cheats and the persistence of symbioses. New Phytol. 177: 849858.

Douglas, A. E. 2010. The Symbiotic Habit. pp. 232. Princeton University Press.

Douglas, A. E. and D. Smith. 1984. The green Hydra symbiosis. VIII. Mechanisms in symbiont regulation. Proceeding R. Soc. London. Ser. B 221: 291-319.

Downs, C. A., E. Kramarsky-Winter, J. Martinez, A. Kushmaro, C. M. Woodley, Y. Loya and G. K. Ostrander. 2009. Symbiophagy as a cellular mechanism for coral bleaching. Autophagy 5: 211-216.

Dunn, S. R., C. E. Schnitzler and V. M. Weis. 2007. Apoptosis and autophagy as mechanisms of dinoflagellate symbiont release during cnidarian bleaching: every which way you lose. Proc. Biol. Sci. 274: 3079-3085.

Dunn, S. R. and V. M. Weis. 2009. Apoptosis as a post-phagocytic winnowing mechanism in a coral-dinoflagellate mutualism. Environ. Microbiol. 11: 268-276.

Enríquez, S., E. R. Méndez and R. I. -Prieto. 2005. Multiple scattering on coral skeletons enhances light absorption by symbiotic algae. Limnol. Oceanogr. 50: 1025-1032.

Ewald, P. W. 1983. Host-parasite relations, vectors, and the evolution of disease severity. Annu. Rev. Ecol. Syst. 14: 465-485.

Ewald, P. W. 1987. Transmission modes and evolution of the parasitism-mutualism continuum. Ann. N. Y. Acad. Sci. 503: 295-306.

Fabricius, K. E., C. Langdon, S. Uthicke, C. Humphrey, S. Noonan, G. De'ath, R. Okazaki, N. Muehllehner, M. S. Glas and J. M. Lough. 2011. Losers and winners in coral reefs acclimatized to elevated carbon dioxide concentrations. Nat. Clim. Chang. 1: 165-169. Nature Publishing Group.

Falkowski, P., Z. Dubinsky, L. Muscatine and L. McCloskey. 1993. Population control in 
symbiotic corals. Bioscience 43: 606-611.

Farrant, P. A., M. A. Borowitzka, R. Hinde and R. J. King. 1987. Nutrition of the temperate Australian soft coral Capnella gaboensis. Mar. Biol. 95: 565-574.

Fautin, D. 2013. Hexacorallians of the World. http//geoportal.kgs.ku.edu/hexacoral/.

Fautin, D. G. 1991. The anemonefish symbiosis: what is known and what is not. Symbiosis 10: 23-46. Balaban Publishers.

Fine, P. E. 1975. Vectors and vertical transmission: an epidemiologic perspective. Ann. N. Y. Acad. Sci. 266: 173-194.

Fitt, W. 2000. Cellular growth of host and symbiont in a cnidarian-zooxanthellar symbiosis. Biol. Bull. 198: 110-120.

Fitt, W. K. 1984. The role of chemosensory behavior of Symbiodinium microadriaticum, intermediate hosts, and host behavior in the infection of coelenterates and molluscs with zooxanthellae. Mar. Biol. 81: 9-17.

Fitt, W. K. 2000. Cellular growth of host and symbiont in a cnidarian-zooxanthellar symbiosis. Biol. Bull. 198: 110-20.

Fitt, W. K., B. E. Brown, M. E. Warner and R. P. Dunne. 2001. Coral bleaching: Interpretation of thermal tolerance limits and thermal thresholds in tropical corals. Coral Reefs 20: 51-65. Springer-Verlag.

Fitt, W. K., S. S. Chang and R. K. Trench. 1981. Motility patterns of different strains of the symbiotic dinoflagellate Symbiodinium (= Gymnodinium) microadriaticum (Freudenthal) in culture. Bull. Mar. Sci. 31: 436-443.

Fitt, W. K., H. J. Spero, J. Halas, M. W. White and J. W. Porter. 1993. Recovery of the coral Montastrea annularis in the Florida Keys after the 1987 Caribbean "bleaching event." Coral Reefs 12: 57-64.

Fitt, W. K. and R. K. Trench. 1983. Endocytosis of the symbiotic dinoflagellate Symbiodinium microadriaticum freudenthal by endodermal cells of the scyphistomae of Cassiopeia xamachana and resistance of the algae to host digestion. J. Cell Sci. 64: 195-212.

Freudenthal, H. D. 1962. Symbiodinium gen. nov. and Symbiodinium microadriaticum sp. nov., a zooxanthella: taxonomy, life cycle, and morphology. J. Protozool. 9: 45-52.

Fujita, T. 2002. Evolution of the lectin-complement pathway and its role in innate immunity. 
Nat. Rev. Immunol. 2: 346-353.

Fujita, T., M. Matsushita and Y. Endo. 2004. The lectin-complement pathway-its role in innate immunity and evolution. Immunol. Rev. 198: 185-202.

Furla, P., D. Allemand, J. M. Shick, C. Ferrier-Pagès, S. Richier, A. Plantivaux, P.-L. Merle and S. Tambutté. 2005. The symbiotic anthozoan: A physiological chimera between alga and animal. Integr. Comp. Biol. 45: 595-604.

Gajigan, A. P. and C. Conaco. 2017. A microRNA regulates the response of corals to thermal stress. Mol. Ecol. 26: 3472-3483.

Gates, R. D., G. Baghdasarian and L. Muscatine. 1992. Temperature stress causes host cell detachment in symbiotic cnidarians: implications for coral bleaching. Biol. Bull. 182: 324332.

Gates, R. D. and P. J. Edmunds. 1999. The physiological mechanisms of acclimatization in tropical reef corals. Am. Zool. 39: 30-43.

Gattuso, J., D. Allemand and M. Frankignoull. 1999. Photosynthesis and calcification at cellular, organismal and community levels in coral reefs: A review on interactions and control by carbonate chemistry. Integr. Comp. Biol. 39: 160-183.

Geurts, R., E. Fedorova and T. Bisseling. 2005. Nod factor signaling genes and their function in the early stages of Rhizobium infection. Curr. Opin. Plant Biol. 8: 346-352.

Gladfelter, E. H. 1983. Spatial and temporal patterns of mitosis in the cells of the axial polyp of the reef roral Acropora cervicornis. Biol. Bull. 165: 811-815.

Godinot, C., C. Ferrier-pagès and R. Grover. 2009. Control of phosphate uptake by zooxanthellae and host cells in the scleractinian coral Stylophora pistillata. Limnol. Oceanogr. 54: 1627-1633.

Goff, L. J. 1982. Symbiosis and parasitism: Another viewpoint. Bioscience 32: 255-256.

Goiran, C., S. Al-Moghrabi, D. Allemand and J. Jaubert. 1996. Inorganic carbon uptake for photosynthesis by the symbiotic coral/dinoflagellate association: I. Photosynthetic performances of symbionts and dependence on sea water bicarbonate. J. Exp. Mar. Bio. Ecol. 199: 207-225.

Goulet, T. L. 2006. Most corals may not change their symbionts. Mar. Ecol. Prog. Ser. 321: 1-7.

Goulet, T. L., C. B. Cook and D. Goulet. 2005. Effect of short-term exposure to elevated 
temperatures and light levels on photosynthesis of different host-symbiont combinations in the Aiptasia pallida-Symbiodinium symbiosis. Limnol. Oceanogr. 50: 1490-1498.

Grajales, A. and E. Rodríguez. 2014. Morphological revision of the genus Aiptasia and the family Aiptasiidae (Cnidaria, Actiniaria, Metridioidea). Zootaxa 3826: 55-100.

Grajales, A. and E. Rodríguez. 2016. Elucidating the evolutionary relationships of the Aiptasiidae, a widespread cnidarian-dinoflagellate model system (Cnidaria: Anthozoa: Actiniaria: Metridioidea). Mol. Phylogenet. Evol. 94: 252-263.

Grover, R., J.-F. J. Maguer, S. Reynaud-vaganay, C. Ferrier-Pagès and C. Ferrier-page. 2002. Uptake of ammonium by the scleractinian coral Stylophora pistillata: Effect of feeding, light, and ammonium concentrations. Limnol. Oceanogr. 47: 782-790.

Gruenberg, J. and F. G. van der Goot. 2006. Mechanisms of pathogen entry through the endosomal compartments. Nat. Rev. Mol. Cell Biol. 7: 495-504.

Harii, S., N. Yasuda, M. Rodriguez-Lanetty, T. Irie and M. Hidaka. 2009. Onset of symbiosis and distribution patterns of symbiotic dinoflagellates in the larvae of scleractinian corals. Mar. Biol. 156: 1203-1212.

Hastings, J. W. 1983. Biological diversity, chemical mechanisms, and the evolutionary origins of bioluminescent systems. J. Mol. Evol. 19: 309-321.

Hawkins, T. D. and S. K. Davy. 2012. Nitric oxide production and tolerance differ among symbiodinium types exposed to heat stress. Plant Cell Physiol. 53: 1889-1898.

Hellinger, J., P. Jägers, M. Donner, F. Sutt, M. D. Mark, B. Senen, R. Tollrian and S. Herlitze. 2017. The flashlight fish anomalops katoptron uses bioluminescent light to detect prey in the dark. PLoS One $\mathbf{1 2 .}$

Hennige, S. J., D. J. Suggett, M. E. Warner, K. E. McDougall and D. J. Smith. 2009. Photobiology of Symbiodinium revisited: bio-physical and bio-optical signatures. Coral Reefs 28: 179-195.

Herre, E. A., N. Knowlton, U. G. Mueller and S. A. Rehner. 1999. The evolution of mutualisms: exploring the paths between conflict and cooperation. Trends Ecol. Evol. 14: $49-53$.

Hillyer, K. E., D. A. Dias, A. Lutz, S. P. Wilkinson, U. Roessner and S. K. Davy. 2017. Metabolite profiling of symbiont and host during thermal stress and bleaching in the coral Acropora aspera. Coral Reefs 36: 105-118. Springer Berlin Heidelberg. 
Hillyer, K. E., S. Tumanov, S. Villas-Bôas and S. K. Davy. 2016. Metabolite profiling of symbiont and host during thermal stress and bleaching in a model cnidarian-dinoflagellate symbiosis. J. Exp. Biol. 219: 516-527, doi:10.1242/jeb.128660.

Hirose, M., R. a. Kinzie and M. Hidaka. 2000. Early development of zooxanthella-containing eggs of the corals Pocillopora verrucosa and P. eydouxi with special reference to the distribution of zooxanthellae. Biol. Bull. 199: 68-75.

Hirose, M., R. Kinzie and M. Hidaka. 2001. Timing and process of entry of zooxanthellae into oocytes of hermatypic corals. Coral Reefs 20: 273-280.

Hoegh-Guldberg, O. and M. Fine. 2004. Low temperatures cause coral bleaching. Coral Reefs 23: 444 .

Hoegh-Guldberg, O., L. R. McCloskey and L. Muscatine. 1987. Expulsion of zooxanthellae by symbiotic cnidarians from the Red Sea. Coral reefs 5: 201-204.

Hoegh-Guldberg, O., P. J. Mumby, a J. Hooten, R. S. Steneck, P. Greenfield, E. Gomez, C. D. Harvell, P. F. Sale, a J. Edwards, K. Caldeira, N. Knowlton, C. M Eakin, R. Iglesias-Prieto, N. Muthiga, R. H. Bradbury, A. Dubi, M. E. Hatziolos. 2007. Coral reefs under rapid climate change and ocean acidification. Science 318: 1737-42.

Hoegh-Guldberg, O. and G. J. Smith. 1989a. The effect of sudden changes in temperature, light and salinity on the population density and export of zooxanthellae from the reef corals Stylophora pistillata (Esper) and Seriatopora hystrix (Dana). J. Exp. Mar. Bio. Ecol. 129: 279-303.

Hoegh-Guldberg, O. and G. Smith. 1989b. Influence of the population density of zooxanthellae and supply of ammonium on the biomass and metabolic characteristics of the reef corals Seriatopora hystrix and Stylophora pistillata. Mar. Ecol. Prog. Ser. 57: 173-186.

Hoegh Guldberg, O. and B. Salvat. 1995. Periodic mass-bleaching and elevated sea temperatures: Bleaching of outer reef slope communities in Moorea, French Polynesia. Mar. Ecol. Prog. Ser. 121: 181-190.

Hofmann, D. K. and B. P. Kremer. 1981. Carbon metabolism and strobilation in Cassiopea andromedea (Cnidaria: Scyphozoa): Significance of endosymbiotic dinoflagellates. Mar. Biol. 65: 25-33.

Hong, M.-C., Y.-S. Huang, P.-C. Song, W.-W. Lin, L.-S. Fang and M.-C. Chen. 2009. Cloning and characterization of ApRab4, a recycling Rab protein of Aiptasia pulchella, and 
its implication in the symbiosome biogenesis. Mar. Biotechnol. 11: 771-785.

Howard, B. 1967. Intestinal micro-organisms of ruminants and other vertabrates. In: Symbiosis, ed. S. M. Henry, Vol. 2. NY: Academic.

Hughes, T. P., a H. Baird, D. R. Bellwood, M. Card, S. R. Connolly, C. Folke, R. Grosberg, O. Hoegh-Guldberg, J. B. C. Jackson, J. Kleypas, J. M. Lough, P. Marshall, M. Nysrtöm, S. R. Palumbi, J. M. Pandolfi, B. Rosen, J. Roughgarden. 2003. Climate change, human impacts, and the resilience of coral reefs. Science 301: 929-33.

Iglesias-Prieto, R., V. H. Beltran, T. C. LaJeunesse, H. Reyes-Bonilla and P. E. Thome. 2004. Different algal symbionts explain the vertical distribution of dominant reef corals in the eastern Pacific. Proc. R. Soc. London, Ser. B Biol. Sci. 271: 1757-1763.

Jackson, A. E. and D. Yellowlees. 1990. Phosphate Uptake by Zooxanthellae Isolated from Corals. Proc. R. Soc. B Biol. Sci. 242: 201-204.

James, E. R. and D. R. Green. 2004. Manipulation of apoptosis in the host-parasite interaction.

Jeong, H. J., Y. Du Yoo, N. S. Kang, A. S. Lim, K. A. Seong, S. Y. Lee, M. J. Lee, K. H. Lee, H. S. Kim, W. Shin, S. W. Nam, W. Yih. 2012. Heterotrophic feeding as a newly identified survival strategy of the dinoflagellate Symbiodinium. PNAS 109: 12604-12609.

Jokiel, P. L. and S. L. Coles. 1977. Effects of temperature on the mortality and growth of Hawaiian reef corals. Mar. Biol. 43: 201-208.

Jolley, E. and D. F. R. . Smith. 1980. The green hydra symbiosis. II. The biology of the establishment of the association. Proceeding R. Soc. London. Ser. B 207: 311-333.

Jones, A. and R. Berkelmans. 2010. Potential costs of acclimatization to a warmer climate: Growth of a reef coral with heat tolerant vs. sensitive symbiont types. PLoS One 5: e10437.

Jones, A. M. and R. Berkelmans. 2011. Tradeoffs to thermal acclimation: energetics and reproduction of a reef coral with heat tolerant Symbiodinium type-D. J. Mar. Biol. 2011: 112.

Jones, A. M., R. Berkelmans, M. J. H. van Oppen, J. C. Mieog and W. Sinclair. 2008. A community change in the algal endosymbionts of a scleractinian coral following a natural bleaching event: field evidence of acclimatization. Proc. Biol. Sci. 275: 1359-65.

Jones, K. M., H. Kobayashi, B. W. Davies, M. E. Taga and G. C. Walker. 2007. How rhizobial symbionts invade plants: The Sinorhizobium - Medicago model. Nat. Rev. 
Microbiol. 5: 619-633.

Jones, R. J. and D. Yellowlees. 1997. Regulation and control of intracellular algae (= zooxanthellae) in hard corals. Philos. Trans. R. Soc. B Biol. Sci. 352: 457-468.

Joyner, J. L., S. M. Peyer and R. W. Lee. 2003. Possible roles of sulfur-containing amino acids in a chemoautotrophic bacterium-mollusc symbiosis. Biol. Bull. 205: 331-338.

Karakashian, S. J. and M. A. Rudzinska. 1981. Inhibition of lysosomal fusion with symbiontcontaining vacuoles in Paramecium bursaria. Exp. Cell Res. 131: 387-393.

Kawaguti, S. and D. Sakumoto. 1948. The effect of light on the calcium deposition of corals. Bull. Ocean. Inst. Taiwan 4: 65-70.

Kellogg, R. B. and J. S. Patton. 1983. Lipid droplets, medium of energy exchange in the symbiotic anemone Condylactis gigantea: a model coral polyp. Mar. Biol. 75: 137-149.

Kemp, D. W., X. Hernandez-Pech, R. Iglesias-Prieto, W. K. Fitt and G. W. Schmidt. 2014a. Community dynamics and physiology of Symbiodinium spp. before, during, and after a coral beaching event. Limnol. Oceanogr. 59: 788-797.

Kenkel, C. and M. V Matz. 2016. Enhanced gene expression plasticity as a mechanism of adaptation to a variable environment in a reef-building coral. bioRxiv 1: doi.org/10.1101/059667.

Keshavmurthy, S., P.-J. Meng, J.-T. Wang, C.-Y. Kuo, S.-Y. Yang, C.-M. Hsu, C.-H. Gan, C.-F. Dai and C. A. Chen. 2014. Can resistant coral- Symbiodinium associations enable coral communities to survive climate change? A study of a site exposed to long-term hot water input. PeerJ 2: e327.

Kimbell, J. R. and M. J. McFall-Ngai. 2004. Symbiont-induced changes in host actin during the onset of a beneficial animal-bacterial association. Appl. Environ. Microbiol. 70: 1434-1441.

Kinzie, R. A., M. Takayama, S. R. Santos and M. A. Coffroth. 2001. The adaptive bleaching hypothesis: Experimental tests of critical assumptions. Biol. Bull. 200: 51-58.

Kinzie, R. and G. Chee. 1979. The effect of different zooxanthellae on the growth of experimentally reinfected hosts. Biol. Bull. 156: 315-327.

Kinzie, R., M. Takayama, S. Santos and M. Coffroth. 2001. The adaptive bleaching hypothesis: experimental tests of critical assumptions. Biol. Bull. 200: 51-58.

Koop, K., D. Booth, a. Broadbent, J. Brodie, D. Bucher, D. Capone, J. Coll, W. Dennison, 
M. Erdmann, P. Harrison, O. Hoegh-Guldberg, P. Hutchings, G. B. Jones, A. W. D. Larkum, J. O'Neil, A. Steven, E. Tentori, S. Ward, J. Williamson, D. Yellowlees. 2001. ENCORE: The effect of nutrient enrichment on coral reefs. Synthesis of results and conclusions. Mar. Pollut. Bull. 42: 91-120.

Kopp, C., M. Pernice, I. Domart-Coulon, C. Djediat, J. E. Spangenberg, D. T. L. Alexander, M. Hignette, T. Meziane and A. Meibom. 2013. Highly dynamic cellular-level response of symbiotic coral to a sudden increase in environmental nitrogen. 4: 1-9.

Koul, A., T. Herget, B. Klebl and A. Ullrich. 2004. Interplay between mycobacteria and host signalling pathways. Nat. Rev. Microbiol. 2: 189-202.

LaJeunesse, T. C. 2002. Diversity and community structure of symbiotic dinoflagellates from Caribbean coral reefs. Mar. Biol. 141: 387-400.

LaJeunesse, T. C., H. R. Bonilla, M. E. Warner, M. Wills, G. W. Schmidt and W. K. Fitt. 2008. Specificity and stability in high latitude eastern Pacific coral-algal symbioses. Limnol. Oceanogr. 53: 719-727.

LaJeunesse, T. C., W. K. W. Loh, R. van Woesik, O. Hoegh-Guldberg, G. W. Schmidt and W. K. Fitt. 2003. Low symbiont diversity in southern Great Barrier Reef corals relative to those of the Caribbean. Limnol. Oceanogr. 48: 2046-2054.

LaJeunesse, T. C., D. T. Pettay, E. M. Sampayo, N. Phongsuwan, B. Brown, D. O. Obura, O. Hoegh-Guldberg and W. K. Fitt. 2010. Long-standing environmental conditions, geographic isolation and host-symbiont specificity influence the relative ecological dominance and genetic diversification of coral endosymbionts in the genus Symbiodinium. J. Biogeogr. 37: 785-800.

LaJeunesse, T. C., R. T. Smith, J. Finney and H. Oxenford. 2009. Outbreak and persistence of opportunistic symbiotic dinoflagellates during the 2005 Caribbean mass coral "bleaching" event. Proc. Biol. Sci. 276: 4139-4148.

LaJeunesse, T., D. Thornhill, E. Cox, F. Stanton, W. Fitt and G. Schmidt. 2004. High diversity and host specificity observed among symbiotic dinoflagellates in reef coral communities from Hawaii. Coral Reefs 23: 596-603. Springer-Verlag.

Leal, M. C., K. Hoadley, D. T. Pettay, A. Grajales, R. Calado and M. E. Warner. 2015. Symbiont type influences trophic plasticity of a model cnidarian-dinoflagellate symbiosis. $J$. Exp. Biol. 218: 858-863. 
Lecointe, A., I. Domart-coulon, A. Paris, A. Meibom and I. Domart-coulon. 2016. Cell proliferation and migration during early development of a symbiotic scleractinian coral. Proc. R. Soc. B 283: 20160206.

Lee, K. H. and E. G. Ruby. 1994. Competition between Vibrio fischeri strains during initiation and maintenance of a light organ symbiosis. J. Bacteriol. 176: 1985-1991.

Lee, M. J., H. J. Jeong, S. H. Jang, S. Y. Lee, N. S. Kang, K. H. Lee, H. S. Kim, D. C. Wham and T. C. LaJeunesse. 2016. Most low-abundance "background" Symbiodinium spp. are transitory and have minimal functional significance for symbiotic corals. Microb. Ecol. 71: $771-783$.

Lenaers, G., L. Maroteaux, B. Michot and M. Herzog. 1989. Dinoflagellates in evolution. A molecular phylogenetic analysis of large subunit ribosomal RNA. J. Mol. Evol. 29: 40-51.

Lesnoff, M. and R. Lancelot. 2012. aod caod: Analysis of overdispersed data. R package version 1.3.

Lesser, M. P. 2004. Experimental biology of coral reef ecosystems. J. Exp. Mar. Bio. Ecol. 300: $217-252$.

Lesser, M. P., M. Stat and R. D. Gates. 2013. The endosymbiotic dinoflagellates (Symbiodinium sp.) of corals are parasites and mutualists. Coral reefs 32: 603-611.

Lewis, J. B. and W. S. Price. 1975. Feeding mechanisms and feeding strategies of Atlantic reef corals. J. Zool. 176: 527-544.

Limpens, E., R. Mirabella, E. Fedorova, C. Franken, H. Franssen, T. Bisseling and R. Geurts. 2005. Formation of organelle-like N2-fixing symbiosomes in legume root nodules is controlled by DMI2. Proc. Natl. Acad. Sci. 102: 10375-10380.

Lin, K., J. Wang and L. Fang. 2000. Participation of glycoproteins on zooxanthellal cell walls in the establishment of a symbiotic relationship with the sea anemone, Aiptasia pulchella. Zool. Stud. 39: 172-178.

Little, A. F., M. J. H. van Oppen and B. L. Willis. 2004. Flexibility in algal endosymbioses shapes growth in reef corals. Science 304: 1492-1494.

Logan, D. D. K., A. C. LaFlamme, V. M. Weis and S. K. Davy. 2010a. Flow-cytometric characterization of the cell-surface glycans of symbiotic dinoflagellates (Symbiodinium spp.). J. Phycol. 46: 525-533. 
Logan, D. D. K., A. C. LaFlamme, V. M. Weis and S. K. Davy. 2010b. Flow-cytometric characterization of the cell-surface glycans of symbiotic dinoflagellates (Symbiodinium app.). J. Phycol. 46: 525-533.

Loram, J. E., H. G. Trapido-Rosenthal and a. E. Douglas. 2007. Functional significance of genetically different symbiotic algae Symbiodinium in a coral reef symbiosis. Mol. Ecol. 16: 4849-4857.

Loya, Y., K. Sakai, Y. Nakano and R. Van Woesik. 2001. Coral bleaching: the winners and the losers. Ecol. Lett. 4: 122-131.

Lutz, A., J. B. Raina, C. A. Motti, D. J. Miller and M. J. H. Van Oppen. 2015. Host coenzyme Q redox state is an early biomarker of thermal stress in the coral Acropora millepora. PLoS One 10: doi:10.1371/journal.pone.0139290.

Madsen, E. B., L. H. Madsen, S. Radutoiu, M. Olbryt, M. Rakwalska, K. Szczyglowski, S. Sato, T. Kaneko, S. Tabata, N. Sandal, et al. 2003. A receptor kinase gene of the LysM type is involved in legume perception of rhizobial signals. Nature 425: 637-640.

Marlow, H. Q. and M. Q. Martindale. 2007. Embryonic development in two species of scleractinian coral embryos: Symbiodinium localization and mode of gastrulation. Evol. Dev. 9: $355-367$.

Marshall, P. A. and A. H. Baird. 2000. Bleaching of corals on the Great Barrier Reef: Differential susceptibilities among taxa. Coral Reefs 19: 155-163.

Matthews, J. L., C. M. Crowder, C. A. Oakley, A. Lutz, U. Roessner, E. Meyer, A. R. Grossman, V. M. Weis and S. K. Davy. 2017. Optimal nutrient exchange and immune responses operate in partner specificity in the cnidarian-dinoflagellate symbiosis. Proc. Natl. Acad. Sci. doi/10.1073/pnas.1710733114 PNAS.

Matthews, J. L., A. E. Sproles, C. A. Oakley, A. R. Grossman, V. M. Weis and S. K. Davy. 2015. Menthol-induced bleaching rapidly and effectively provides experimental aposymbiotic sea anemones (Aiptasia sp.) for symbiosis investigations. J. Exp. Biol. 219: 306-310.

McAuley, P. 1985a. The cell cycle of symbiotic Chlorella. I. The relationship between host feeding and algal cell growth and division. J. Cell Sci. 77: 225-239.

McAuley, P. 1985b. The cell cycle of symbiotic Chlorella. II. The effect of continuous darkness. J. Cell Sci. 77: 241-253. 
McAuley, P. J. 1982. Temporal relationships of host cell and algal mitosis in the green Hydra symbiosis. J. Cell Sci. 58: 423-431.

McAuley, P. J. 1981. Control of cell division of the intracellular Chlorella symbionts in green Hydra. J. Cell Sci. 47: 197-206.

McAuley, P. J. and C. B. Cook. 1994. Effects of host feeding and dissolved ammonium on cell division and nitrogen status of zooxanthellae in the hydroid Myrionema amboinense. Mar. Biol. 121: 343-348.

McAuley, P. J. and D. C. Smith. 1982. The green Hydra symbiosis. V. stages in the intracellular recognition of algal symbionts by digestive cells. Proc. R. Soc. London. Ser. B. Biol. Sci. 216: $7-23$.

McAuley, P. and L. Muscatine. 1986. The cell cycle of symbiotic Chlorella. IV. DNA content of algae slowly increases during host starvation of green Hydra. J. Cell Sci. 85: 73-84.

McCloskey, L. R., T. G. Cove and E. A. Verde. 1996. Symbiont expulsion from the anemone Anthopleura elegantissima (Brandt) (Cnidaria; Anthozoa). J. Exp. Mar. Bio. Ecol. 195: 173186.

McFall-Ngai, M., E. A. C. Heath-Heckman, A. A. Gillette, S. M. Peyer and E. A. Harvie. 2012. The secret languages of coevolved symbioses: Insights from the Euprymna scolopesVibrio fischeri symbiosis. Semin. Immunol. 24: 3-8.

Meints, R. and R. Pardy. 1980. Quantitative demonstration of cell surface involvement in a plant-animal symbiosis: lectin inhibition of reassociation. J. Cell Sci. 43: 239-251.

Mergaert, P., T. Uchiumi, B. Alunni, G. Evanno, A. Cheron, O. Catrice, A.-E. Mausset, F. Barloy-Hubler, F. Galibert, A. Kondorosi, et al. 2006b. Eukaryotic control on bacterial cell cycle and differentiation in the Rhizobium-legume symbiosis. Proc. Natl. Acad. Sci. U. S. A. 103: 5230-5235.

Mieog, J. C., J. L. Olsen, R. Berkelmans, S. a. Bleuler-Martinez, B. L. Willis and M. J. H. van Oppen. 2009. The roles and interactions of symbiont, host and environment in defining coral fitness. PLoS One 4: e6364.

Mieog, J. C., M. J. H. Van Oppen, N. E. Cantin, W. T. Stam and J. L. Olsen. 2007. Real-time PCR reveals a high incidence of Symbiodinium clade D at low levels in four scleractinian corals across the Great Barrier Reef: Implications for symbiont shuffling. Coral Reefs 26: 449-457. 
Miller, D. J. and D. Yellowlees. 1989. Inorganic nitrogen uptake by symbiotic marine cnidarian: a critical review. Proceeding R. Soc. London. Ser. B 237: 109-125.

Mohamed, A. R., V. Cumbo, S. Harii, C. Shinzato, C. X. Chan, M. A. Ragan, D. G. Bourne, B. L. Willis, E. E. Ball, N. Satoh, et al. 2016. The transcriptomic response of the coral Acropora digitifera to a competent Symbiodinium strain: The symbiosome as an arrested early phagosome. Mol. Ecol. 25: 3127-3141.

Muller-Parker, G., C. Cook and C. D'Elia. 1990. Feeding affects phosphate fluxes in the symbiotic sea anemone Aiptasia pallida. Mar. Ecol. Prog. Ser. 60: 283-290.

Muller-Parker, G. and C. D'Elia. 1997. Interactions between corals and their symbiotic algae. In: Birkeland, C. (Ed.), Life and death of coral reefs. pp. 96-113. Chapman \& Hall, New York.

Muller-Parker, G. and S. K. Davy. 2001. Temperate and tropical algal-sea anemone symbioses. Invertebr. Biol. 120: 104-123.

Muscatine, L. 1967. Glycerol excretion by symbiotic algae from corals and tridacna and its control by the host. Science 156: 516-519.

Muscatine, L. 1990. The role of symbiotic algae in carbon and energy flux in reef corals. Ecosyst. world 25: 75-87. Elsevier.

Muscatine, L. and E. Cernichiari. 1969. Assimilation of photosynthetic products of zooxanthellae by a reef coral. Biol. Bull. 137: 506-523.

Muscatine, L. and C. F. D'Elia. 1978. The uptake, retention, and release of ammonium by reef corals. Limnol. Oceanogr. 23: 725-734.

Muscatine, L., P. Falkowski and Z. Dubinsky. 1983. Carbon budgets in symbiotic associations. In: Endocytobiol. II. Schwemmler W \& Schenk HEA, eds., pp. 649-658. Walter de Gruyter and Co., Berlin.

Muscatine, L., P. G. Falkowski, Z. Dubinsky, P. A. Cook and L. R. McCloskey. 1989. The effect of external nutrient resources on the population dynamics of zooxanthellae in a reef coral. Proc. R. Soc. B Biol. Sci. 236: 311-324.

Muscatine, L., P. G. Falkowski, J. W. Porter and Z. Dubinsky. 1984. Fate of photosynthetic fixed carbon in light- and shade-adapted colonies of the symbiotic coral Stylophora pistillata. Proc. R. Soc. B Biol. Sci. 222: 181-202. 
Muscatine, L., R. D. Gates and I. Lafontaine. 1994. Do symbiotic dinoflagellates secrete lipid droplets? Limnol. Oceanogr. 39: 925-929.

Muscatine, L., C. Goiran, L. Land, J. Jaubert, J. P. Cuif and D. Allemand. 2005. Stable isotopes $\left(\delta^{13} \mathrm{C}\right.$ and $\left.\delta^{15} \mathrm{~N}\right)$ of organic matrix from coral skeleton. Proc. Natl. Acad. Sci. U. S. A. 102: $1525-1530$.

Muscatine, L. and C. Hand. 1958. Direct evidence for the transfer of materials from symbiotic algae to the tissues of a Coelenterate. Proc. Natl. Acad. Sci. U. S. A. 44: 1259-63.

Muscatine, L. and R. Pool. 1979. Regulation of number of intracellular algae. Proceeding $R$. Soc. London. Ser. B, Biol. Sci. 204: 131-139.

Muscatine, N. and L. Neckelmann. 1983. Regulatory mechanisms maintaining the HydraChlorella symbiosis. Proc. R. Soc. London 219: 193-210.

Neckelmann, N., L. Muscatine, N. Muscatine and L. Neckelmann. 1983. Regulatory mechanisms maintaining the Hydra-Chlorella symbiosis. Proc. R. Soc. London. Ser. B, Biol. Sci. 219: 193-210.

Neubauer, E. F., A. Z. Poole, P. Neubauer, O. Detournay, K. Tan, S. K. Davy and V. M. Weis. 2017. A diverse host thrombospondin-type-1 repeat protein repertoire promotes symbiont colonization during establishment of cnidarian-dinoflagellate symbiosis. Elife 6: DOI: 10.7554/eLife.24494.

Núñez-Pons, L., I. Bertocci and G. Baghdasarian. 2017. Symbiont dynamics during thermal acclimation using cnidarian-dinoflagellate model holobionts. Mar. Environ. Res. 130: 303314.

Nyholm, S. V and M. J. McFall-Ngai. 2004. The winnowing: establishing the squid-Vibrio symbiosis. Nat. Rev. Microbiol. 2: 632-642.

Nyholm, S. V. and M. J. McFall-Ngai. 2003. Dominance of Vibrio fischeri in secreted mucus outside the light organ of Euprymna scolopes: The first site of symbiont specificity. Appl. Environ. Microbiol. 69: 3932-3937.

Nyholm, S. V, B. Deplancke, H. R. Gaskins, M. A. Apicella and M. J. McFall-Ngai. 2002. Roles of Vibrio fischeri and nonsymbiotic bacteria in the dynamics of mucus secretion during symbiont colonization of the Euprymna scolopes light organ. Appl. Environ. Microbiol. 68: 5113-5122.

Oakley, C. A., E. Durand, S. P. Wilkinson, L. Peng, V. M. Weis, A. R. Grossman and S. K. 
Davy. 2017. Thermal shock induces host proteostasis disruption and endoplasmic reticulum stress in the model symbiotic cnidarian Aiptasia. J. Proteome Res. 16: 2121-2134.

Oldroyd, G. E. D. 2013. Speak, friend, and enter: Signalling systems that promote beneficial symbiotic associations in plants. Nat. Rev. Microbiol. 11: 252-263.

Oldroyd, G. E. D. and J. A. Downie. 2004. Calcium, kinases and nodulation signalling in legumes. Nat. Rev. Mol. Cell Biol. 5: 566-576.

Oldroyd, G. E. and J. A. Downie. 2006. Nuclear calcium changes at the core of symbiosis signalling. Curr. Opin. Plant Biol. 9: 351-357.

Oliver, T. A. and S. R. Palumbi. 2011. Many corals host thermally resistant symbionts in hightemperature habitat. Coral Reefs 30: 241-250.

Parrin, A. P., T. L. Goulet, M. A. Yaeger, L. S. Bross, C. S. McFadden and N. W. Blackstone. 2016. Symbiodinium migration mitigates bleaching in three octocoral species. J. Exp. Mar. Bio. Ecol. 474: 73-80. Elsevier B.V.

Parrin, A. P., K. L. Harmata, S. E. Netherton, M. A. Yaeger, L. S. Bross and N. W. Blackstone. 2012. Within-colony migration of symbionts during bleaching of octocorals. Biol. Bull. 223: 245-256.

Patton, J. S. and J. E. Burris. 1983. Lipid synthesis and extrusion by freshly isolated zooxanthellae (symbiotic algae). Mar. Biol. 75: 131-136.

Pawlowski, J., M. Holzmann, J. F. Fahrni, X. Pochon and J. J. Lee. 2001. Molecular identification of algal endosymbionts in large miliolid foraminifera: 2. Dinofiagellates. $J$. Eukaryot. Microbiol. 48: 368-373.

Paxton, C. W., S. K. Davy and V. M. Weis. 2013. Stress and death of cnidarian host cells play a role in cnidarian bleaching. J. Exp. Biol. 216: 2813-20.

Pearse, V. B. and L. Muscatine. 1971. Role of symbiotic algae (zooxanthellae) in coral calcification. Biol. Bull. 141: 350-363.

Peng, S.-E., W.-N. U. Chen, H.-K. Chen, C.-Y. Lu, A. B. Mayfield, L.-S. Fang and C.-S. Chen. 2011. Lipid bodies in coral-dinoflagellate endosymbiosis: proteomic and ultrastructural studies. Proteomics 11: 3540-3555.

Peng, S. E., Y. B. Wang, L. H. Wang, W. N. U. Chen, C. Y. Lu, L. S. Fang and C. S. Chen. 2010. Proteomic analysis of symbiosome membranes in cnidaria - dinoflagellate 
endosymbiosis. Proteomics 10: 1002-1016.

Penterman, J., R. P. Abo, N. J. De Nisco, M. F. F. Arnold, R. Longhi, M. Zanda and G. C. Walker. 2014. Host plant peptides elicit a transcriptional response to control the Sinorhizobium meliloti cell cycle during symbiosis. Proc. Natl. Acad. Sci. 111: 3561-3566.

Pernice, M., S. R. Dunn, T. Miard, S. Dufour, S. Dove and O. Hoegh-Guldberg. 2011. Regulation of apoptotic mediators reveals dynamic responses to thermal stress in the reef building coral Acropora millepora. PLoS One 6: doi:10.1371/journal.pone.0016095.

Pernice, M., A. Meibom, A. Van Den Heuvel, C. Kopp, I. Domart-Coulon, O. HoeghGuldberg and S. Dove. 2012. A single-cell view of ammonium assimilation in coraldinoflagellate symbiosis. Isme J 6: 1314-1324.

Perret, X., C. Staehelin and W. J. Broughton. 2000. Molecular basis of symbiotic promiscuity. Microbiol. Mol. Biol. Rev. 64: 180-201.

Pettay, D. T. and T. C. Lajeunesse. 2009. Microsatellite loci for assessing genetic diversity, dispersal and clonality of coral symbionts in "stress-tolerant" clade D Symbiodinium. Mol. Ecol. Resour. 9: 1022-1025.

Pettay, D. T., D. C. Wham, R. T. Smith, R. Iglesias-Prieto and T. C. LaJeunesse. 2015. Microbial invasion of the Caribbean by an Indo-Pacific coral zooxanthella. Proc. Natl. Acad. Sci. 112: 7513-7518.

Pochon, X. and R. D. Gates. 2010. A new Symbiodinium clade (Dinophyceae) from soritid foraminifera in Hawai'i. Mol. Phylogenet. Evol. 56: 492-497.

Pochon, X., J. I. Montoya-Burgos, B. Stadelmann and J. Pawlowski. 2006. Molecular phylogeny, evolutionary rates, and divergence timing of the symbiotic dinoflagellate genus Symbiodinium. Mol. Phylogenet. Evol. 38: 20-30.

Poland, D. M. and M. A. Coffroth. 2017. Trans-generational specificity within a cnidarianalgal symbiosis. Coral Reefs 36: 119-129.

Putnam, H. M., M. Stat, X. Pochon, R. R. D. Gates, M. Medina, E. Weil, L. D. L. Mydlarz, V. Dale, M. Vanderwel, D. Purves, et al. 2012. Endosymbiotic flexibility associates with environmental sensitivity in scleractinian corals. Proc. R. Soc. B Biol. Sci. 279: 4352-4361.

R Core Team. 2016. R: A Language and Environment for Statistical Computing. Vienna, Austria: R Foundation for Statistical Computing. 
Rädecker, N., C. Pogoreutz, C. R. Voolstra, J. Wiedenmann and C. Wild. 2015. Nitrogen cycling in corals: The key to understanding holobiont functioning? Trends Microbiol. 23: 490-497.

Raff, M. 1998. Cell suicide for beginners. Nature 396: 119-122.

Ragni, M., R. L. Airs, S. J. Hennige, D. J. Suggett, M. E. Warner and R. J. Geider. 2010. PSII photoinhibition and photorepair in Symbiodinium (Pyrrhophyta) differs between thermally tolerant and sensitive phylotypes. Mar. Ecol. Prog. Ser. 406: 57-70.

Rahav, O., Z. Dubinsky, Y. Achituv and P. G. Falkowski. 1989. Ammonium metabolism in the zooxanthellate coral, Stylophora pistillata. Proceeding R. Soc. London. Ser. B 236: 325337.

Reimer, A. A. 1971. Observations on the relationships between several species of tropical zoanthids (Zoanthideas coelenterata) and their zooxanthellae. J. Exp. Mar. Bio. Ecol. 7: 207-214.

Reynaud, S., P. Martinez, F. Houlbrèque, I. Billy, D. Allemand and C. Ferrier-Pagès. 2009. Effect of light and feeding on the nitrogen isotopic composition of a zooxanthellate coral: Role of nitrogen recycling. Mar. Ecol. Prog. Ser. 392: 103-110.

Reynolds, J. M., B. U. Bruns, W. K. Fitt and G. W. Schmidt. 2008. Enhanced photoprotection pathways in symbiotic dinoflagellates of shallow-water corals and other cnidarians. Proc. Natl. Acad. Sci. U. S. A. 105: 13674-13678.

Reynolds, W., J. Schwarz and V. Weis. 2000. Symbiosis-enhanced gene expression in cnidarian-algal associations: cloning and characterization of a cDNA, sym32, encoding a possible cell adhesion protein. Comp. Biochem. Physiol. 126: 33-44.

Richier, S., P. L. Merle, P. Furla, D. Pigozzi, F. Sola and D. Allemand. 2003. Characterization of superoxide dismutases in anoxia- and hyperoxia-tolerant symbiotic cnidarians.

Robison, J. D. and M. E. Warner. 2006. Differential impacts of photoacclimation and thermal stress on the photobiology of four different phylotypes of Symbiodinium (pyrrhophyta). $J$. Phycol. 42: 568-579.

Rodriguez-Lanetty, M. and E. Wood-Charlson. 2006. Dynamics of infection and localization of dinoflagellate endosymbionts in larvae of the coral Fungia scutaria during the onset of symbiosis. Mar. Biol.

Roth, M. S. 2014. The engine of the reef: photobiology of the coral-algal symbiosis. Front. 
Microbiol. 5: doi: 10.3389/fmicb.2014.00422.

Roughgarden, J. 1975. Evolution of marine symbiosis - a simple cost-benefit model. Ecology 56: $1201-1208$.

Rowan, R. 1998. Review—diversity and ecology of zooxanthellae on coral reefs. J. Phycol. 34: 407-417.

Rowan, R. 2004. Coral bleaching: thermal adaptation in reef coral symbionts. Nature 430: 742.

Sachs, J. L., C. J. Essenberg and M. M. Turcotte. 2011. New paradigms for the evolution of beneficial infections. Trends Ecol. Evol. 26: 202-209.

Sachs, J. L. and E. L. Simms. 2006. Pathways to mutualism breakdown. Trends Ecol. Evol. 21: $585-592$.

Sachs, J. L., R. G. Skophammer and J. U. Regus. 2011. Evolutionary transitions in bacterial symbiosis. Proc. Natl. Acad. Sci. U. S. A. 108: 10800-10807.

Sachs, J. L., M. Ehinger and E. L. Simms. 2010. Origins of cheating and loss of symbiosis in wild Bradyrhizobium. J. Evol. Biol. 23: 1075-1089.

Sachs, J. L. and T. P. Wilcox. 2006a. A shift to parasitism in the jellyfish symbiont Symbiodinium microadriaticum. Proc. Biol. Sci. 273: 425-429.

Sachs, J. L. and T. P. Wilcox. 2006b. A shift to parasitism in the jellyfish symbiont Symbiodinium microadriaticum. Proc. R. Soc. B Biol. Sci. 273: 425-429.

Sacks, D. and A. Sher. 2002. Evasion of innate immunity by parasitic protozoa.

Salih, A., A. Larkum, G. Cox, M. Kühl and O. Hoegh-Guldberg. 2000. Fluorescent pigments in corals are photoprotective. Nature 408: 850-853.

Sampayo, E. M., L. Franceschinis, O. Hoegh-Guldberg and S. Dove. 2007. Niche partitioning of closely related symbiotic dinoflagellates. Mol. Ecol. 16: 3721-3733.

Sampayo, E. M., T. Ridgway, P. Bongaerts and O. Hoegh-Guldberg. 2008. Bleaching susceptibility and mortality of corals are determined by fine-scale differences in symbiont type. Proc. Natl. Acad. Sci. U. S. A. 105: 10444-10449.

Santos, S. R., T. L. Shearer, A. R. Hannes and M. A. Coffroth. 2004. Fine-scale diversity and specificity in the most prevalent lineage of symbiotic dinoflagellates (Symbiodinium, Dinophyceae) of the Caribbean. Mol. Ecol. 13: 459-469. 
Schmidt, E. W., A. Y. Obraztsova, S. K. Davidson, D. J. Faulkner and M. G. Haygood. 2000. Identification of the antifungal peptide-containing symbiont of the marine sponge Theonella swinhoei as a novel $\delta$-proteobacterium, " Candidatus Entotheonella palauensis." Mar. Biol. 136: 969-977.

Schmiege, P. F. P., C. C. D'Aloia and P. M. Buston. 2017. Anemonefish personalities influence the strength of mutualistic interactions with host sea anemones. Mar. Biol. 164.

Schoenberg, D. A. and R. K. Trench. 1980a. Genetic variation in Symbiodinium (=Gymnodinium) microadriaticum freudenthal, and specificity in its symbiosis with marine invertebrates. III. Specificity and infectivity of Symbiodinium microadriaticum. Proc. $R$. Soc. London Ser. B Biol. Sci. 207: 445-460.

Schoenberg, D. A. and R. K. Trench. 1980b. Genetic variation in Symbiodinium (=Gymnodinium) microadriaticum freudenthal, and specificity in its symbiosis with marine invertebrates. I. Isoenzyme and soluble protein patterns of axenic cultures of Symbiodinium microadriaticum. Proc. R. Soc. B Biol. Sci. 207: 405-427.

Schoenberg, D. A. and R. K. Trench. 1980c. Genetic variation in Symbiodinium microadriaticum (=Gymnodinium) and specificity in its symbiosis with Marine Invertebrates II. morphological variation in Symbiodinium microadriaticum. SO - Proc. $R$. Soc. London - Ser. B Biol. Sci. 207(1169). 1980. 429-444.

Sheppard, C. R. C., S. K. Davy and G. M. Pilling. 2009. The biology of coral reefs. pp. 384. Oxford University Press, Oxford.

Silverstein, R. N., A. M. S. Correa, A. C. Baker, X. Pochon, R. D. Gates, A. C. Baker, M. Rodriguez-Lanetty, D. A. Krupp, V. M. Weis, T. C. LaJeunesse, et al. 2012. Specificity is rarely absolute in coral-algal symbiosis: implications for coral response to climate change. Proc. Biol. Sci. 279: 2609-18.

Silverstein, R. N., R. Cunning and A. C. Baker. 2015. Change in algal symbiont communities after bleaching, not prior heat exposure, increases heat tolerance of reef corals. Glob. Chang. Biol. 21: 236-249.

Smith, G. and L. Muscatine. 1999. Cell cycle of symbiotic dinoflagellates : variation in G1 phase-duration with anemone nutritional status and macronutrient supply in the Aiptasia pulchella \pm Symbiodinium pulchrorum symbiosis. Mar. Biol. 134: 405-418.

Sogin, E. M., H. M. Putnam, P. E. Anderson and R. D. Gates. 2016. Metabolomic signatures 
of increases in temperature and ocean acidification from the reef-building coral, Pocillopora damicornis. Metabolomics 12: 71.

Sorokin, Y. I. 1992. Phosphorus metabolism in coral reef communities: exchange between the water column and bottom biotopes.

Sproles, A. E., N. L. Kirk, S. A. Kitchen, C. A. Oakley, A. R. Grossman, V. M. Weis and S. K. Davy. 2018. Phylogenetic characterization of transporter proteins in the cnidariandinoflagellate symbiosis. Mol. Phylogenet. Evol. 120: 307-320.

Stambler, N. and Z. Dubinsky. 1987. Energy relationships between Anemonia sulcata and its endosymbiotic zooxanthellae. Symbiosis 3: 233-248.

Starzak, D. E., R. G. Quinnell, M. R. Nitschke and S. K. Davy. 2014. The influence of symbiont type on photosynthetic carbon flux in a model cnidarian-dinoflagellate symbiosis. Mar. Biol. 161: 711-724.

Stat, M., C. E. Bird, X. Pochon, L. Chasqui, L. J. Chauka, G. T. Concepcion, D. Logan, M. Takabayashi, R. J. Toonen and R. D. Gates. 2011. Variation in Symbiodinium ITS2 sequence assemblages among coral colonies. PLoS One 6: e15854.

Stat, M., D. Carter and O. Hoegh-guldberg. 2006. The evolutionary history of Symbiodinium and scleractinian hosts-symbiosis, diversity, and the effect of climate change. Perspect. Plant Ecol. Evol. Syst. 8: 23-43.

Stat, M. and R. D. Gates. 2011. Clade D Symbiodinium in Scleractinian Corals: A "Nugget" of hope, a selfish opportunist, an Oominous Ssign, or all of the above? J. Mar. Biol. 2011: 1-9.

Stat, M., W. K. W. Loh, T. C. LaJeunesse, O. Hoegh-Guldberg and D. A. Carter. 2009. Stability of coral-endosymbiont associations during and after a thermal stress event in the southern Great Barrier Reef. Coral Reefs 28: 709-713.

Stat, M., E. Morris and R. D. Gates. 2008. Functional diversity in coral-dinoflagellate symbiosis. Proc. Natl. Acad. Sci. 105: 9256-9261.

Steele, R. and N. Goreau. 1977. The breakdown of symbiotic zooxanthellae in the sea anemone Phyllactis (= Oulactis) flosculifera (Actiniaria). J. Zool. 181: 421-437.

Steen, G. and L. Muscatine. 1984. Daily budgets of photosynthetically fixed carbon in symbiotic zoanthids. Biol. Bull. 167: 477-487.

Stimson, J. and R. A. Kinzie. 1991. The temporal pattern and rate of release of zooxanthellae 
from the reef coral Pocillopora damicornis (Linnaeus) under nitrogen-enrichment and control conditions. J. Exp. Mar. Bio. Ecol. 153: 63-74.

Suggett, D. J., M. E. Warner, D. J. Smith, P. Davey, S. Hennige and N. R. Baker. 2008. Photosynthesis and production of hydrogen peroxide by Symbiodinium (Pyrrhophyta) phylotypes with different thermal tolerances. J. Phycol. 44: 948-956.

Sun, J., V. Cardoza, D. M. Mitchell, L. Bright, G. Oldroyd and J. M. Harris. 2006. Crosstalk between jasmonic acid, ethylene and Nod factor signaling allows integration of diverse inputs for regulation of nodulation. Plant J. 46: 961-970.

Sun, Y., E. D. LaSota, A. G. Cecere, K. B. LaPenna, J. Larios-Valencia, M. S. Wollenberg and T. Miyashiro. 2016. Intraspecific competition impacts Vibrio fischeri strain diversity during initial colonization of the squid light organ. Appl. Environ. Microbiol. 82: 3082-91.

Sutton, D. C. and O. Hoegh-Guldberg. 1990. Host-zooxanthella interactions in four temperate marine invertebrate symbioses: Assessment of effect of host extracts on symbionts. Biol. Bull. 178: 175-186.

Tambutte, E., D. Allemand and J. Jaubert. 1995. The Stylophora pistillata microcolony: A model for studying calcium transport process during coral biomineralization. Bull. L'Institut Oceanogr. 79-87.

Taylor, C., L. Muscatine and D. Jefferson. 1989. Maintenance and breakdown of the HydraChlorella symbiosis: a computer model. Proc. R. Soc. London. B. Biol. Sci. 238: 277-289.

Taylor, D. 1974. Symbiotic marine algae: taxonomy and biological fitness. In: Vernberg, W.B. (Ed.), University of South Carolina Press, Columbia.

Taylor, M. W., R. Radax., D. Steger., M. Wagner. 2007. Sponge-associated microorganisms: Evolution, ecology, and biotechnological potential. Microbiol. Mol. Biol. Rev. 71: 295-347.

Tchernov, D., H. Kvitt, L. Haramaty, T. S. Bibby, M. Y. Gorbunov, H. Rosenfeld and P. G. Falkowski. 2011. Apoptosis and the selective survival of host animals following thermal bleaching in zooxanthellate corals. Proc. Natl. Acad. Sci. 108: 9905-9909.

Tentori, E. and D. Allemand. 2006. Light-enhanced calcification and dark decalcification in isolates of the soft coral Cladiella sp. during tissue recovery. Biol. Bull. 211: 193-202.

Thompson, J. N. 1988. Variation in interspecific interactions. Annu. Rev. Ecol. Syst. 19: 65-87. 
Thornberry, N. A. and Y. Lazebnik. 1998. Caspases : Enemies within. Science. 281: 13121316.

Thornhill, D. J., D. W. Kemp, B. U. Bruns, W. K. Fitt and G. W. Schmidt. 2008. Correspondence between cold tolerance and temperate biogeography in a western Atlantic Symbiodinium (Dinophyta) lineage.

Thornhill, D. J., T. C. LaJeunesse, D. W. Kemp, W. K. Fitt and G. W. Schmidt. 2006. Multiyear, seasonal genotypic surveys of coral-algal symbioses reveal prevalent stability or postbleaching reversion. Mar. Biol. 148: 711-722.

Thornhill, D. J., Y. Xiang, D. T. Pettay, M. Zhong and S. R. Santos. 2013. Population genetic data of a model symbiotic cnidarian system reveal remarkable symbiotic specificity and vectored introductions across ocean basins. Mol. Ecol. 22: 4499-4515.

Titlyanov, E., T. Titlyanova, V. Leletkin, J. Tsukahara, R. van Woesik and K. Yamazato. 1996. Degradation of zooxanthellae and regulation of their density in hermatypic corals. Mar. Ecol. Prog. Ser. 139: 167-178.

Toft, C. and S. G. E. Andersson. 2010. Evolutionary microbial genomics: insights into bacterial host adaptation. Nat. Rev. Genet. 11: 465-475. Nature Publishing Group.

Tremblay, P., R. Grover, J. F. Maguer, M. Hoogenboom and C. Ferrier-Pagès. 2014. Carbon translocation from symbiont to host depends on irradiance and food availability in the tropical coral Stylophora pistillata. Coral Reefs 33: 1-13.

Trench, R. 1979. The cell biology of plant-animal symbiosis. Annu. Rev. Plant Physiol. 30: 485531.

Trench, R. 1997. Diversity of symbiotic dinoflagellates and the evolution of microalgalinvertebrate symbioses. Proc 8th Int Coral Reef Symp 2: 1275-1286.

Trench, R. K. 1971a. The physiology and biochemistry of zooxanthellae symbiotic with marine Coelenterates. II. Liberation of fixed formulaC by zooxanthellae in vitro. Proc. R. Soc. B Biol. Sci. 177: 237-250.

Trench, R. K. 1971b. The physiology and biochemistry of zooxanthellae symbiotic with marine Coelenterates. I. The assimilation of photosynthetic products of zooxanthellae by two marine oelenterates. Proc. R. Soc. B Biol. Sci. 177: 225-235.

Trench, R. K. 1971c. The physiology and biochemistry of zooxanthellae symbiotic with marine Coelenterates. III. The effect of homogenates of host tissues on the excretion of 
photosynthetic products in vitro by zooxanthellae from two marine Coelenterates. Proc. $R$. Soc. B Biol. Sci. 177: 251-264.

Trench, R. K. 1974. Nutritional potentials in Zoanthus sociathus (Coelenterata, Anthozoa). Helgoländer Wissenschaftliche Meeresuntersuchungen 26: 174-216.

Unson, M. D., N. D. Holland and D. J. Faulkner. 1994. A brominated secondary metabolite synthesized by the cyanobacterial symbiont of a marine sponge and accumulation of the crystalline metabolite in the sponge tissue. Mar. Biol. 119: 1-11.

Van Brussel, A. A. N., R. Bakhuizen, P. C. Van Spronsen, H. P. Spaink, T. Tak, B. J. J. Lugtenberg and J. W. Kijne. 1992. Induction of pre-infection thread structures in the leguminous host plant by mitogenic lipo-oligosaccharides of rhizobium. Science 257: 7072.

Van Woesik, R., K. Sakai, A. Ganase and Y. Loya. 2011. Revisiting the winners and the losers a decade after coral bleaching. Mar. Ecol. Prog. Ser. 434: 67-76.

Vance, J. E. 2010. Transfer of cholesterol by the NPC team. Cell Metab. 12: 105-106.

Venn, A. A., J. E. Loram and A. E. Douglas. 2008. Photosynthetic symbioses in animals. $J$. Exp. Bot. 59: 1069-1080.

Venn, A. A., E. Tambutté, S. Lotto, D. Zoccola, D. Allemand and S. Tambutté. 2009. Imaging intracellular $\mathrm{pH}$ in a reef coral and symbiotic anemone. Proc. Natl. Acad. Sci. U. S. A. 106: 16574-9.

von Holt, C. and M. von Holt. 1968. The secretion of organic compounds by zooxanthellae isolated from various types of Zoanthus. Comp. Biochem. Physiol. 24: 83-92.

Voolstra, C. R., J. A. Schwarz, J. Schnetzer, S. Sunagawa, M. K. Desalvo, A. M. Szmant, M. A. Coffroth and M. Medina. 2009. The host transcriptome remains unaltered during the establishment of coral-algal symbioses: FAST TRACK. Mol. Ecol. 18: 1823-1833.

Wakefield, T. S., M. a. Farmer and S. C. Kempf. 2000. Revised description of the fine structure of in situ "Zooxanthellae" genus Symbiodinium. Biol. Bull. 199: 76-84.

Wakefield, T. S. and S. C. Kempf. 2001. Development of host- and symbiont-specific monoclonal antibodies and confirmation of the origin of the symbiosome membrane in a cnidarian-dinoflagellate symbiosis. Biol. Bull. 200: 127-143.

Wang, J.-T., Y.-Y. Chen, K. S. Tew, P.-J. Meng and C. a Chen. 2012. Physiological and 
biochemical performances of menthol-induced aposymbiotic corals. PLoS One 7: e46406.

Wang, J. T. and A. E. Douglas. 1999. Essential amino acid synthesis and nitrogen recycling in an alga-invertebrate symbiosis. Mar. Biol. 135: 219-222.

Wang, L. H., Y. H. Liu, Y. M. Ju, Y. Y. Hsiao, L. S. Fang and C. S. Chen. 2008. Cell cycle propagation is driven by light-dark stimulation in a cultured symbiotic dinoflagellate isolated from corals. Coral Reefs 27: 823-835.

Webster, N. S. and M. W. Taylor. 2012. Marine sponges and their microbial symbionts: love and other relationships. Environ. Microbiol. 14: 335-46.

Weeks, A. R., M. Turelli, W. R. Harcombe, K. T. Reynolds and A. A. Hoffmann. 2007. From parasite to mutualist: Rapid evolution of Wolbachia in natural populations of Drosophila. PLoS Biol. 5: 0997-1005.

Wegley, L., R. Edwards, B. Rodriguez-Brito, H. Liu and F. Rohwer. 2007. Metagenomic analysis of the microbial community associated with the coral Porites astreoides. Environ. Microbiol. 9: 2707-2719.

Weis, V. M. 1993. Effect of dissolved inorganic carbon concentration on thepPhotosynthesis of the symbiotic sea-anemone Aiptasia-pulchella Carlgren: Role of carbonic anhydrase. J. Exp. Mar. Bio. Ecol. 174: 209-225.

Weis, V. M. 2008. Cellular mechanisms of Cnidarian bleaching: stress causes the collapse of symbiosis. J. Exp. Biol. 211: 3059-3066.

Weis, V. M., S. K. Davy, O. Hoegh-Guldberg, M. Rodriguez-Lanetty and J. R. Pringle. 2008. Cell biology in model systems as the key to understanding corals. Trends Ecol. Evol. 23: $369-376$.

Weis, V., W. Reynolds, M. DeBoer and D. Krupp. 2001. Host-symbiont specificity during onset of symbiosis between the dinoflagellates Symbiodinium spp. and planula larvae of the scleractinian coral Fungia scutaria. Coral Reefs 20: 301-308.

Whitehead, L. F. and a E. Douglas. 2003. Metabolite comparisons and the identity of nutrients translocated from symbiotic algae to an animal host. J. Exp. Biol. 206: 3149-3157.

Wier, A. M., S. V. Nyholm, M. J. Mandel, R. P. Massengo-Tiasse, A. L. Schaefer, I. Koroleva, S. Splinter-BonDurant, B. Brown, L. Manzella, E. Snir, et al. 2010. Transcriptional patterns in both host and bacterium underlie a daily rhythm of anatomical and metabolic change in a beneficial symbiosis. Proc. Natl. Acad. Sci. 107: 2259-2264. 
Wickham, H. 2009. Ggplot2 : elegrant graphics for data analysis. Springer-Verlag, New York.

Wicks, L. C., E. Sampayo, J. P. A. Gardner and S. K. Davy. 2010. Local endemicity and high diversity characterise high-latitude coral-Symbiodinium partnerships. Coral Reefs 29: 9891003.

Widder, E. A. 2010. Bioluminescence in the ocean: origins of biological, chemical, and ecological diversity. Science. 328: 704-708.

Wilkerson, F. P., G. Muller-Parker and L. Muscatine. 1983. Temporal patterns of cell division in unicellular algae. Physiol. Bases Phytoplankt. Ecol. 28: 1009-14.

Wilkinson, C. R. 1983. Net primary productivity in coral reef sponges. Science 219: 410-412.

Woesik, R. van, K. Sakai, A. Ganase and Y. Loya. 2011. Revisiting the winners and the losers a decade after coral bleaching. Mar. Ecol. Prog. Ser. 434: 67-76.

Wolfowicz, I., S. Baumgarten, P. A. Voss, E. A. Hambleton, C. R. Voolstra, M. Hatta and A. Guse. 2016. Aiptasia sp. larvae as a model to reveal mechanisms of symbiont selection in cnidarians. Sci. Rep. 6: 32366. Nature Publishing Group.

Wood-Charlson, E. M., L. L. Hollingsworth, D. a. Krupp and V. M. Weis. 2006. Lectin/glycan interactions play a role in recognition in a coral/dinoflagellate symbiosis. Cell. Microbiol. 8: 1985-1993.

Xiang, T., E. A. Hambleton, J. C. Denofrio, J. R. Pringle and A. R. Grossman. 2013. Isolation of clonal axenic strains of the symbiotic dinoflagellate Symbiodinium and their growth and host specificity. J. Phycol. 49: 447-458.

Yamashita, H., G. Suzuki, T. Hayashibara and K. Koike. 2011. Do corals select zooxanthellae by alternative discharge? Mar. Biol. 158: 87-100.

Yellowlees, D., T. A. V Rees and W. Leggat. 2008. Metabolic interactions between algal symbionts and invertebrate hosts. Plant. Cell Environ. 31: 679-694.

Yuyama, I., T. Nakamura, T. Higuchi and M. Hidaka. 2016. Different stress tolerances of juveniles of the coral Acropora tenuis associated with clades C1 and D Symbiodinium. Zool. Stud. 55: 1-9. 


\title{
Appendix 1
}

Reference: Biol. Bull. 234: 000-000. (February 2018)

C 2018 The University of Chicago

\section{Symbiont Identity Influences Patterns of Symbiosis Establishment, Host Growth, and Asexual Reproduction in a Model Cnidarian- Dinoflagellate Symbiosis}

\author{
YASMIN GABAY ${ }^{1}$, VIRGINIA M. WEIS ${ }^{2}$, AND SIMON K. DAVY, ${ }^{1, *}$ \\ ${ }^{1}$ School of Biological Sciences, Victoria University of Wellington, Kelburn Parade, Wellington 6140, \\ New Zealand; and ${ }^{2}$ Department of Integrative Biology, Oregon State University, \\ Corvallis, Oregon 97331
}

\begin{abstract}
The genus Symbiodinium is physiologically diverse and so may differentially influence symbiosis establishment and function. To explore this, we inoculated aposymbiotic individuals of the sea anemone Exaiptasia pallida (commonly referred to as Aiptasia), a model for coral symbiosis, with one of five Symbiodinium species or types (S. microadriaticum, S. minutum, phylotype C3, S. trenchii, or S. voratum). The spatial pattern of colonization was monitored over time via confocal microscopy, and various physiological parameters were measured to assess symbiosis functionality. Anemones rapidly formed a symbiosis with the homologous symbiont, S. minutum, but struggled or failed to form a long-lasting symbiosis with Symbiodinium C3 or S. voratum, respectively. Symbiodinium microadriaticum and $S$. trenchii were successful but reached their peak density two weeks after $S$. minutum. The spatial pattern of colonization was identical for all Symbiodinium taxa that were ultimately successful, starting in the oral disk and progressing to the tentacles, before the symbionts invaded the column and, finally, the pedal disk. In all cases, proliferation through the anemone's tentacles was patchy, suggesting that symbionts were being expelled into the gastrovascular cavity and re-phagocytosed by the host. However, the timing of these various spatial events differed between the different Symbiodinium taxa. Furthermore, S. microadriaticum and S. trenchii were less beneficial to the host, as indicated by lower rates of photosynthesis, anemone growth, and pedal laceration. This

Received 8 September 2017; Accepted 7 December 2017; Published online XX XX 2018.

* To whom correspondence should be addressed. E-mail: simon.davy@ vuw.ac.nz.

Abbreviations: DCMU, 3-(3,4-dichlorophenyl)-1,1-dimethylurea; P:R, ratio of hourly gross photosynthesis to respiration.
\end{abstract}

study enhances our understanding of the link between symbiont identity and the performance of the overall symbiosis, which is important for understanding the potential establishment and persistence of novel host-symbiont pairings. Importantly, we also provide a baseline for further studies on this topic with the globally adopted Aiptasia model system.

\section{Introduction}

Among the most significant marine mutualisms are those between cnidarians and their photosynthetic dinoflagellate symbionts (Roth, 2014). These interactions, in particular, between anthozoan cnidarians (e.g., corals and sea anemones) and dinoflagellates of the genus Symbiodinium, underpin the existence and success of coral reef ecosystems (Little et al., 2004; Wang et al., 2012; Lesser et al., 2013).

The genus Symbiodinium is very diverse, consisting of nine clades (A-I) and numerous subclades (types) and species (LaJeunesse, 2002; Pochon and Gates, 2010) that display considerable physiological diversity (Schoenberg and Trench, 1980a; Fitt et al., 1981; Chang et al., 1983; Iglesias-Prieto et al., 2004; Robison and Warner, 2006; Hennige et al., 2009). Given this, different symbiont taxa can differentially affect host and, hence, holobiont (i.e., the entire symbiotic entity) performance. For example, in corals, some members of clades A and $\mathrm{D}$ can be less beneficial to their hosts than members of clade $C$, with lower rates of photosynthetic carbon fixation and translocation to the host and reduced host fitness (Mieog et al., 2009; Leal et al., 2015). Alongside host phylotype and the prevailing environmental conditions, symbiont identity may therefore determine the fate of the symbiosis (Loram et al., 2007; Yuyama et al., 2016). 
Such observations have led to the proposal that several Symbiodinium taxa might exhibit more opportunistic, and even parasitic, traits than others (Stat and Gates, 2011; Lesser et al., 2013; Pettay et al., 2015; Silverstein et al., 2015). Starzak et al. (2014) tested this idea more broadly by modeling carbon fluxes in the model symbiotic anemone Aiptasia when colonized by a range of different Symbiodinium taxa. These models suggested that the usual homologous symbiont ( $S$. minutum, internal transcribed spacer region 2 [ITS2] type B1) forms a more beneficial symbiosis with Aiptasia than do several nonnative heterologous symbiont types, such as A1.4, E, and F5.2; Symbiodinium E and F5.2 were, on occasion, even observed to cause host mortality (Starzak et al., 2014). However, direct measures of host growth and reproductive output, to corroborate the models of Starzak and coworkers, have not been performed. Indeed, we still know relatively little about the behavior and physiology of homologous and heterologous Symbiodinium taxa, in general, during symbiosis establishment, including whether they exhibit similar spatial patterns of uptake and host colonization. Such information is important for understanding the events that might occur during the establishment of novel host-symbiont pairings, as could potentially occur after a coral bleaching event. Furthermore, such details are important for understanding the drivers of host-symbiont specificity, that is, the reasons why many hosts are found in association with only one or a few Symbiodinium taxa and vice versa (Baker, 2003; LaJeunesse et al., 2004; Coffroth et al., 2010).

This study addressed these knowledge gaps using the sea anemone Exaiptasia pallida (Agassiz in Verrill, 1864), commonly referred to as Aiptasia, a widely adopted model system for the study of reef-building corals and the cnidariandinoflagellate symbiosis (Weis et al., 2008; Baumgarten et al., 2015). The current study measured the influence of symbiont diversity on host colonization dynamics, as well as host performance. We employed five different Symbiodinium species or types, representing a wide range of clades (A-E) and physiologies and including the free-living and relatively heterotrophic $S$. voratum (type E; Jeong et al., 2012) and the thermally tolerant but opportunistic $S$. trenchii (type D1a; Pettay and Lajeunesse, 2009). Specifically, we asked whether symbiont identity affects (1) symbiont cell proliferation, (2) colonization pattern inside the host, (3) photosynthetic performance, and (4) host fitness. In doing so, we provide not only further insight into the cellular events that occur during host colonization by symbiotic dinoflagellates and the physiological implications of symbiont diversity but also a baseline from which to conduct more detailed studies of symbiosis establishment and function with the model Aiptasia system.

\section{Materials and Methods}

\section{Experimental organisms}

A clonal culture of the symbiotic sea anemone Aiptasia (culture ID: NZ1), originally from the Indo-Pacific region, was grown in the lab at $25^{\circ} \mathrm{C}$ and an irradiance of $100 \mu \mathrm{mol}$ photons $\mathrm{m}^{-2} \mathrm{~s}^{-1}$ (light/dark cycle of $12 \mathrm{h:12} \mathrm{h}$ ) and fed twice weekly with freshly hatched Artemia nauplii. To generate aposymbiotic (i.e., symbiont-free) anemones, animals were incubated in a solution of $0.27 \mathrm{mmol} 1^{-1}$ menthol/filtered seawater (FSW; $0.22 \mu \mathrm{m}-\mathrm{FSW}$ ) for $8 \mathrm{~h}$, after which the menthol/ FSW was removed and the anemones were incubated in $5 \mu \mathrm{mol} 1^{-1}$ 3-(3,4-dichlorophenyl)-1,1-dimethylurea (DCMU)/ FSW overnight, as described by Matthews et al. (2015), with the treatment being repeated daily for 4 wk or until no symbionts were present, as determined by confocal microscopy (IX81, Olympus, Auckland, New Zealand; 635 nm laser, 655755-nm emission filter).

Cultured Symbiodinium from five clades were used as inoculates (Table 1). Species names of these dinoflagellates are used where available; otherwise, the ITS2 type is given. The algae were subcultured from long-term $(>5 \mathrm{y})$ laboratory stocks and grown in silica-free $\mathrm{f} / 2$ medium (Sigma-Aldrich, Auckland, New Zealand) at $25^{\circ} \mathrm{C}$ and an irradiance of $100 \mu \mathrm{mol}$ photons $\mathrm{m}^{-2} \mathrm{~s}^{-1}$ on a light/dark cycle of $12 \mathrm{~h}: 12 \mathrm{~h}$. All cultures were sampled for experimental use during the log phase of growth.

\section{Inoculation of aposymbiotic anemones with symbionts}

Following 1 wk of starvation, a total of 312 aposymbiotic anemones ( $n=60$ anemones for each Symbiodinium type/ species; $n=12$ uninoculated anemones as a negative control) of similar size (2-3-mm oral disk diameter) were transferred to 400-ml clear plastic jars (one anemone per jar) filled with FSW and allowed to settle for $3 \mathrm{~d}$. Each algal culture was diluted in $25 \mathrm{ml} \mathrm{FSW}$ and 1 drop of Artemia nauplii suspension added, to give a final concentration of $\sim 1 \times 10^{6}$ cells ml $^{-1}$. The anemones were inoculated with one of the five different symbiont cultures by pipetting with a glass pipette $1 \mathrm{ml}$ of this dinoflagellate suspension onto the oral disk of each anemone. Colonization was then monitored over a period of $8 \mathrm{wk}$, with sampling at 1, 2, 4, 6, and 8 wk after inoculation. During this time, the anemones were fed twice weekly with Artemia nauplii, with each feeding followed by a water change. Seawater temperature was maintained at $25{ }^{\circ} \mathrm{C}$ in a controlledtemperature room, and irradiance was held at $~ 100 \mu \mathrm{mol}$ photons $\mathrm{m}^{-2} \mathrm{~s}^{-1}$ on a light/dark cycle of $12 \mathrm{~h}: 12 \mathrm{~h}$.

\section{Symbiont proliferation}

Spatial pattern of colonization. To determine the effect of symbiont identity on the spatial pattern of host colonization, 10 randomly selected anemones from each treatment were chosen at each sampling point and assessed via chlorophyll autofluorescence measured with a confocal microscope (details in Experimental organisms, above). A drop of relaxation solution (50\% $0.37 \mathrm{~mol} \mathrm{l}^{-1} \mathrm{MgCl}_{2}$ in $\mathrm{dH}_{2} \mathrm{O}$ and $50 \% \mathrm{FSW}$ ) containing $1.5 \%$ of agar was added to a subset of the sampled anemones on a fluorodish (World Precision Instruments, Sar- 
Table 1

Identity and geographical origin of Symbiodinium cultures and the original host species if known

\begin{tabular}{llll}
\hline \hline Culture ID & \multicolumn{1}{c}{ Original host source } & Geographical location & Symbiodinium species \\
\hline FlAp2 & Exaiptasia pallida & Long Key, Florida & S. minutum \\
CCMP2467 & Stylophora pistillata & Gulf of Aqaba & S. microadriaticum \\
Mp & Mastigias papua & Palau & Symbiodinium sp. \\
Ap2 & Unknown anemone sp. & Okinawa & A 1 trenchii \\
CCMP421 & Free living (host, if any, unknown) & Wellington Harbour, New Zealand & S. voratum \\
\hline
\end{tabular}

asota, FL) ( $n=5$ per treatment), and z-stack images were generated (section thickness $<10 \mu \mathrm{m}$ ). The multiple z-stack sections were then summed into a single photo, regions of interest were marked, and fluorescence was measured with ImageJ software (ver. 1.48; National Institutes of Health, Bethesda, $\mathrm{MD})$. The following equation was used to calculate fluorescence intensity as a proxy for algal number:

$$
\text { correct fluorescence }=\operatorname{IntDen}_{\mathrm{S}}-\left(\text { areas }_{\mathrm{S}}-\text { mean }_{\mathrm{B}}\right),
$$

where IntDen $=$ fluorescence measured using ImageJ, $\mathrm{S}=$ sample, and B = background.

Quantification of symbionts during colonization. Following confocal microscopy, each anemone was homogenized separately in $500 \mu \mathrm{l}$ of FSW (IKA T-10 tissue lyser, ThermoFisher Scientific) and the homogenate centrifuged (Sigma 3$16 \mathrm{k}$ ) for $5 \mathrm{~min}$ at $400 \times g$ to separate algal cells from anemone tissue. A $100-\mu \mathrm{l}$ aliquot was removed from the supernatant (host fraction) for protein determination and the remaining supernatant discarded. The pellet containing the dinoflagellate cells was re-suspended in $200 \mu \mathrm{l} \mathrm{FSW.} \mathrm{A} \mathrm{50-} \mu$ l aliquot was added to $50 \mu$ l of dimethyl sulfoxide buffer for DNA sequencing (see DNA sequencing, below), and the remaining $150 \mu \mathrm{l}$ was used for algal cell counts. The samples were stored at $-20{ }^{\circ} \mathrm{C}$ until further analysis. Host protein content was determined via the Bradford assay (Bradford, 1976), and cell counting was performed with a hemocytometer (Improved Neubauer) with six replicate counts per sample. Cell density (symbionts per milligram protein) was then calculated.

\section{Photophysiology and host performance}

Photosynthesis and respiration of the host-symbiont partnership. Maximum gross photosynthetic and dark respiratory $\mathrm{O}_{2}$ fluxes were measured for all host-symbiont partnerships at the end of the 8-wk experiment ( $n=4$ for each treatment). Individual anemones were placed at $25^{\circ} \mathrm{C}$ in a $10-\mathrm{ml}$ glass chamber, fitted with a magnetic stir bar protected by nylon mesh, filled with FSW, and sealed by a glass lid with a rubber O-ring, into which an oxygen electrode (FIBOX 3 fiber-optic oxygen meter; PreSens, Regensburg, Germany) was inserted. Animals were allowed to settle for $30 \mathrm{~min}$ prior to the beginning of the experiment, with the chamber remaining unsealed and hence open to the air during this time. The respiration rate $\left(\mathrm{ml} \mathrm{O}_{2} \mathrm{hr}^{-1}\right.$ ) was measured in darkness for an hour, followed by $1 \mathrm{~h}$ of illumination by a 150-W Thorn parabolic aluminized reflector lamp 38 at $400 \mu \mathrm{mol}$ photons $\mathrm{m}^{-2} \mathrm{~s}^{-1}$. Each anemone was then homogenized in $500 \mu \mathrm{l}$ of FSW and host protein content determined as described before. The rates of gross photosynthesis and respiration were normalized to host protein, and the ratio of hourly gross photosynthesis to respiration $(\mathrm{P}: \mathrm{R})$ was calculated as a proxy for the autotrophic potential of the symbiosis.

Host growth and asexual reproduction. Host growth between the start and the end of the experiment (i.e., $8 \mathrm{wk}$ after inoculation) was estimated via the change in oral disk diameter, assuming a starting diameter of $2-3 \mathrm{~mm}(n=10$ for each treatment). At the final time point, anemones were incubated in their containers in $\mathrm{MgCl}_{2}$ relaxation solution (see Spatial pattern of colonization, above) until they were not responsive to disturbance, and then the containers were placed onto millimetric graph paper for measurement of the oral disk diameter. Throughout the duration of the experiment, asexual reproduction was measured as the number of pedal lacerates generated by each anemone between the start of the experiment and the sampling point ( $n=10$ for each treatment and time point).

\section{DNA sequencing}

To confirm that symbiont populations in animals matched the populations used for inoculation, symbiont identity was verified for each culture and a subset of inoculated anemone samples ( $n=3$ samples per culture or sample). Symbiodinium samples containing dimethyl sulfoxide (see Quantification of symbionts during colonization, above) were re-suspended, and $10 \mu \mathrm{l}$ of each sample was added to a new tube containing $50 \mathrm{mg}$ of glass beads (G1152-10G, Sigma-Aldrich) and placed in a TissueLyser (Qiagen, Bio-Strategy) bead beater for $1 \mathrm{~min}$ at $50 \mathrm{~Hz}$. Ninety microliters of $\mathrm{ddH}_{2} \mathrm{O}$ was added, and lysed cell material was pelleted at $16,100 \times g$ for $10 \mathrm{~min}$ at $4{ }^{\circ} \mathrm{C}$. A $50-\mu 1$ aliquot of the resulting supernatant was transferred to a new tube containing $50 \mu \mathrm{l}$ of cold molecular-grade isopropanol, and samples were re-pelleted as described above. Samples were washed twice in $200 \mu$ l of $70 \%$ ethanol, centrifuged, and then dried at room temperature. Fifty microliters of 
$1 \mathrm{~mol}^{-1}$ Tris ( $\mathrm{pH} 8$ ) were added to each tube, and samples were further extracted by bead beating at $30 \mathrm{~Hz}$ for 1 min and then stored at $-20{ }^{\circ} \mathrm{C}$ until further analysis. Polymerase chain reaction (PCR) was performed using the following Symbiodinium-specific ITS2 primers: forward primer (ITS2infor) 5'-GAATTGCAGA ACTCCGTG-3' and reverse primer (ITS2CLAMP) 5'-CGCCCGCCGC GCCCCGCGCC CGTCCCGCCG CCCCCGCCC GGGATCCATA TGCTTAAGTT CAGCGGGT-3' . Reactions were performed using the MyTaq Mix in a total volume of $25 \mu \mathrm{l}$ with an amplification profile consisting of 1 cycle of $3 \mathrm{~min}$ at $95^{\circ} \mathrm{C}$; 40 cycles of $15 \mathrm{~s}$ at $95{ }^{\circ} \mathrm{C}, 15 \mathrm{~s}$ at $56^{\circ} \mathrm{C}$, and $10 \mathrm{~s}$ at $72{ }^{\circ} \mathrm{C}$; and a final hold temperature of $4{ }^{\circ} \mathrm{C}$. PCR products were sequenced by Macrogen (Seoul, South Korea). Sequences were aligned using Geneious Pro, version 4.8.5, and a BLAST search was performed in the National Centre for Biotechnology Information (NCBI) database to identify the sequences. In all cases, the expected genotypes were present at both the beginning and the end of the experiment. Multiple genotypes were never detected in any sample.

\section{Statistical analysis}

ANOVA followed by either Tukey or Bonferroni post hoc tests was performed on the data by using STATISTICA, version 10, and SPSS, except in the following cases. Log transformation was conducted on symbiont density data to achieve a normal distribution, followed by Welch's ANOVA, because constant variance could not be assumed. Welch's ANOVA was also performed on dark respiration data for the same reason. This test was followed by Games-Howell post hoc analysis. A $\chi^{2}$ test was conducted on asexual reproduction data to test the effect of time $\times$ algal identity. Results are expressed as mean \pm standard error (SE).

\section{Results}

All Symbiodinium taxa initially colonized Aiptasia. There was no cross-contamination of symbionts between treatments (data not shown), and aposymbiotic anemones remained symbiontfree throughout the experiment.

\section{Symbiont proliferation in hosts}

Symbiont cell density (algal cells mg host protein ${ }^{-1}$ ) was significantly different between anemones inoculated with different Symbiodinium taxa (Fig. 1; Welch's ANOVA, $F_{19,58}=$ 130.087, $P<0.0001)$. The homologous $S$. minutum was the most successful at colonizing hosts, reaching its peak density 2 wk after inoculation and achieving a maximum density that was 13 -fold higher than that of $S$. trenchii, the second most successful symbiont, at that same time point (Games-Howell post hoc, $P<0.0001$ ). Between weeks 2 and 8 , the density of $S$. minutum decreased by $62 \%$ to $3.35 \times 10^{6} \pm 3.18 \times$ $10^{5}$ cells mg protein $^{-1}$, which was still significantly higher

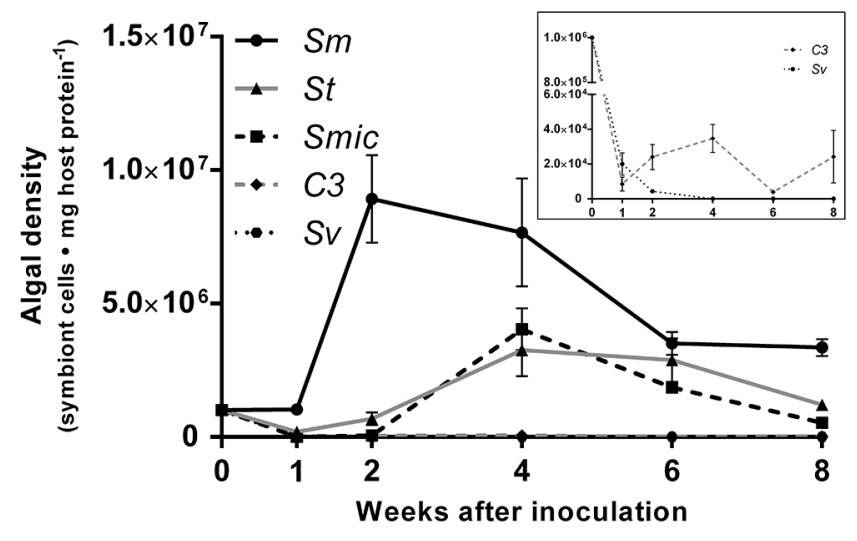

Figure 1. Colonization success in host Aiptasia varied with symbiont identity. Symbiont density (symbiont cells mg host protein ${ }^{-1}$ ) in host anemones was quantified for 8 weeks after inoculation $(n=10$ anemones per treatment per time point). Points are means \pm SE. C3, Symbiodinium phylotype C3; Sm, S. minutum; Smic, S. microadriaticum; St, S. trenchii; Sv, S. voratum.

than that of all other symbionts (Games-Howell post hoc, $P<0.006$ ). Symbiodinium microadriaticum and $S$. trenchii populations exhibited a slower initial increase in density than $S$. minutum, but by week 4 their densities peaked at levels similar to those of $S$. minutum (Games-Howell post hoc, $P>0.05$ for both comparisons; Fig. 1); their densities then declined during the remainder of the experiment by $71 \%$ and $58 \%$, respectively, so that they were again significantly lower than the density of $S$. minutum (Games-Howell post hoc, $P<0.001$ ). Symbiodinium $\mathrm{C} 3$ and $S$. voratum were the least successful at colonization. The population density of C3 was significantly less than that of $S$. minutum and S. trenchii (Games-Howell post hoc, $P<0.05$ ) throughout the experiment and significantly less than $S$. microadriaticum from week 4 onward. In comparison, $S$. voratum initially colonized the host, but after 2 wk its density declined dramatically, such that by week 4 and thereafter, no symbionts were observed (Fig. 1).

The different Symbiodinium taxa showed similar spatial patterns of colonization through host tissues, albeit at different temporal rates (Fig. 2A-C). Colonization in the tentacles did not progress smoothly from the tentacle base to the tip; rather, it was patchier, with punctuated bursts of algal growth in spatially disparate portions of the tentacle (Fig. 2A, B). Furthermore, the timing of these various spatial events differed substantially between the different Symbiodinium (Fig. 2C), consistent with the strength of overall colonization, as shown in Figure 1. Of particular note, the relatively unsuccessful Symbiodinium C3 only ever colonized the oral disk and tentacles and by week 8 appeared in the oral disk alone, suggesting that colonization was failing.

\section{Photophysiology and host performance}

Photosynthesis and respiration of the host-symbiont partnership. Gross photosynthesis ( $\mathrm{mg} \mathrm{O}_{2} \mathrm{mg}_{\text {protein }}{ }^{-1} \mathrm{hr}^{-1}$ ) was significantly different when Aiptasia formed a symbiosis with 

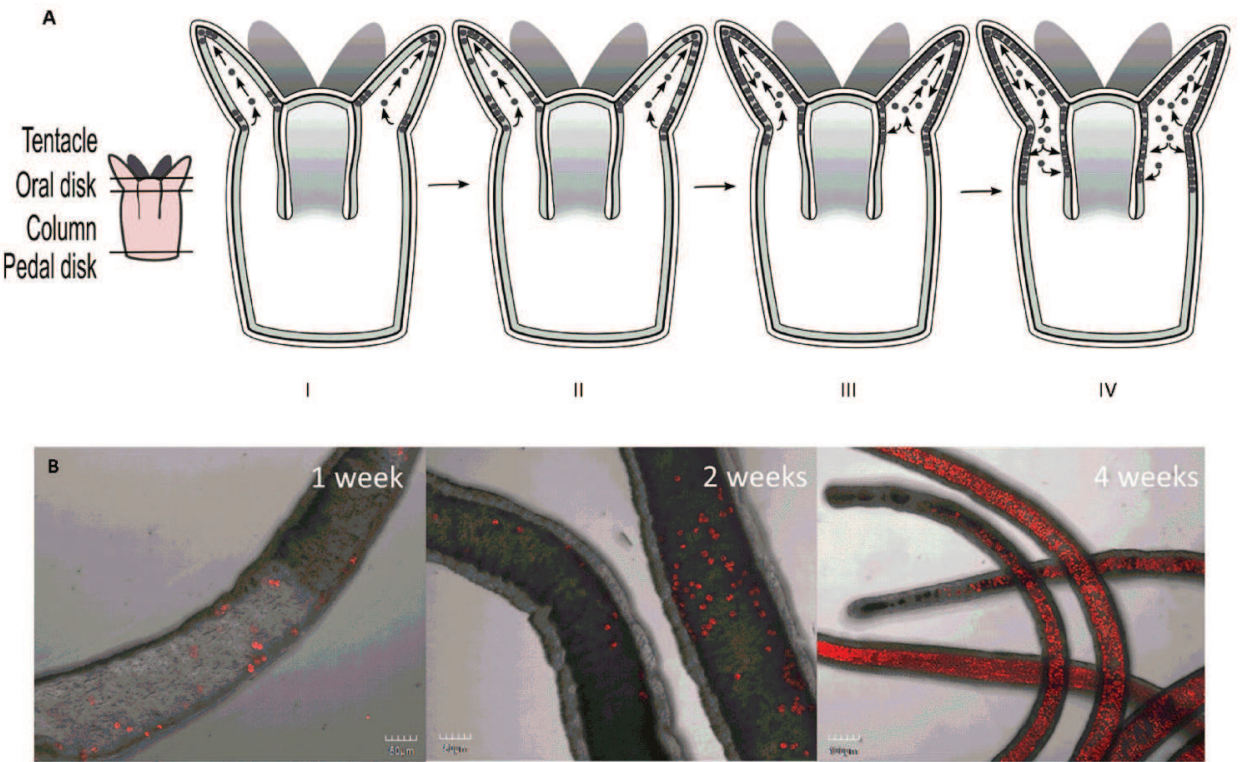

c

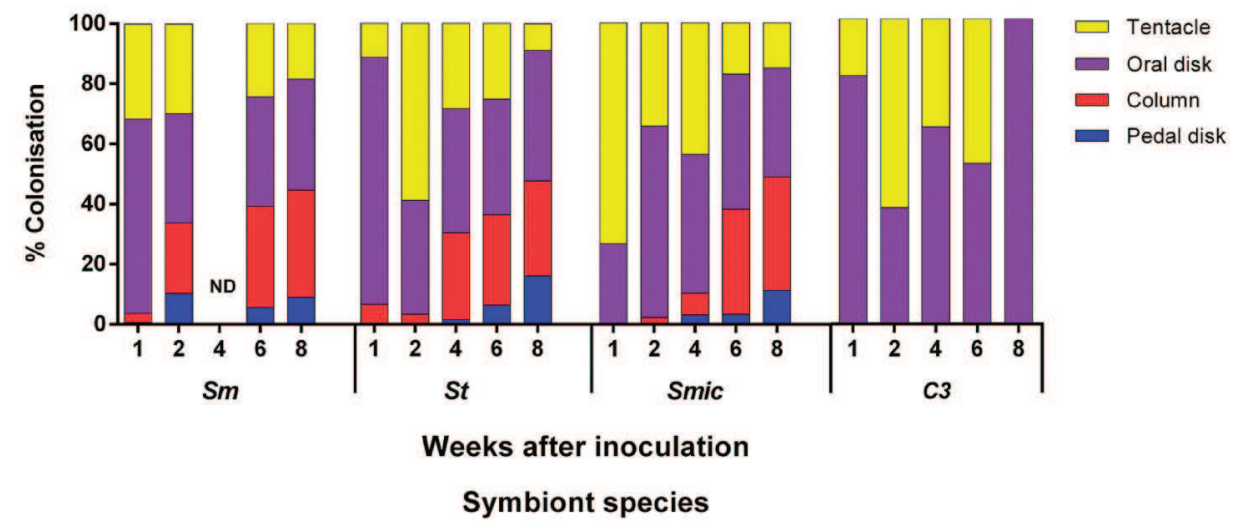

Figure 2. The spatial pattern of symbiont colonization in Aiptasia is similar for different symbionts. Spatial pattern and colonization across four body sections in the anemone host-tentacles, oral disk, column, and pedal disk (A) — quantified for 8 weeks after inoculation ( $n=2-6$ anemones per treatment and time point). (A) Schematic representation. (I) Symbionts taken up by the anemone and concentrated in the oral disk. Proliferation continues to the tentacles and exhibits a patchy pattern, suggesting symbiont migration via the gastrovascular cavity. (II) Colonization of the tentacles continues through mitotic division and gastrovascular migration. (III) Tentacles are fully colonized, and the symbiont cells begin to colonize the upper part of the column. (IV) The symbionts continue to colonize the column from top to bottom, ultimately reaching the pedal disk. (B) Confocal images of tentacle colonization by Symbiodinium trenchii over 4 weeks. Symbiodinium trenchii rather than S. minutum is shown here, since $S$. trenchii was slower to colonize the host and hence the spatial patterns are clearer across time. (C) Spatial pattern of colonization by different symbionts, presented as the percentage of total symbiont chlorophyll fluorescence occurring in the four different body regions of the anemone over time. C3, Symbiodinium phylotype C3; Sm, S. minutum; Smic, S. microadriaticum; St, S. trenchii; Sv, S. voratum.

the different Symbiodinium species or types (one-way ANOVA, iment, anemones containing $S$. minutum or $S$. trenchii photosynthesized at a similar rate, as did those anemones containing S. microadriaticum or type $\mathrm{C} 3$; however, the photosynthetic rate with the former two symbionts was faster than that with the latter two (Tukey post hoc, $P<0.01$ for all comparisons).
In contrast, dark respiration rate $\left(\mathrm{mg} \mathrm{O}_{2} \mathrm{mg}_{\text {protein }}{ }^{-1} \mathrm{hr}^{-1}\right.$ ) was not affected by symbiont identity (Fig. 3A; Welch's ANOVA, $F_{4.5 .5}=3.043, P>0.05$ ). As a result, there was a significant difference in the $\mathrm{P}: \mathrm{R}$ ratio between anemones hosting the different symbionts at the end of the experiment (1-way ANOVA, $F_{3,10}=11.420, P<0.05$ ), with $S$. minutum-colonized anemones having a significantly higher ratio than those con- 
A
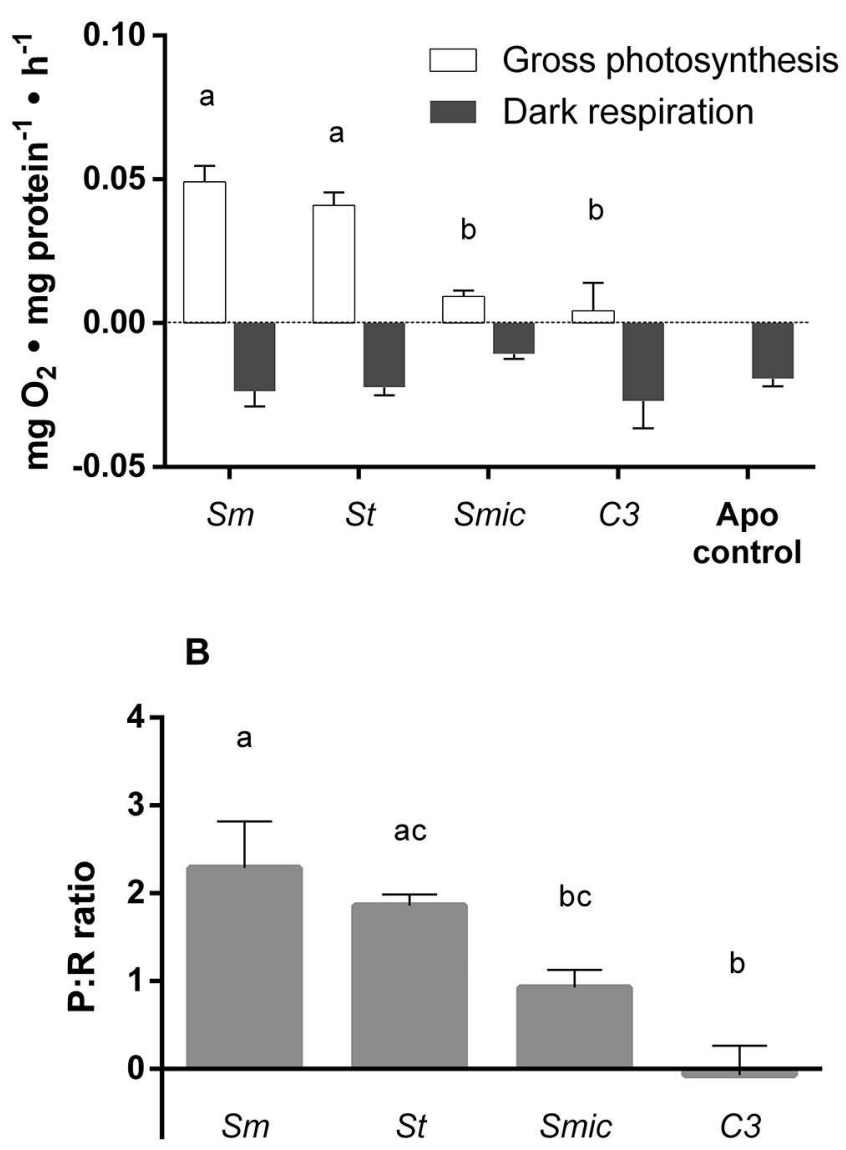

\section{Symbiodinium species}

Figure 3. Gross photosynthesis and dark respiration of the holobiont varied with symbiont identity. Photophysiological performance $\left(\mathrm{mg} \mathrm{O}_{2} \mathrm{mg}\right.$ protein ${ }^{-1} \mathrm{hr}^{-1}$ ) of the holobiont was quantified 8 weeks after inoculation. (A) Gross photosynthesis (white bars) and total symbiosis dark respiration (gray bars). (B) Ratio of hourly gross photosynthesis to respiration (P: R ratio). Bars represent means $\pm \mathrm{SE} ; n=3-4$ anemones per treatment. Different letters above bars indicate significant differences between treatments $(P<$ 0.05). Apo, aposymbiotic; C3, Symbiodinium phylotype C3; Sm, S. minutum; Smic, S. microadriaticum; St, S. trenchii; Sv, S. voratum.

taining S. microadriaticum or C3 (Tukey post hoc, $P<0.05$ for both comparisons) but not $S$. trenchii (Fig. 3B).

Host growth and asexual reproduction. Host growth, estimated at the end of the experiment from the oral disk diameter, was significantly impacted by Symbiodinium identity (1-way ANOVA, $\left.F_{5,44}=19.820, P<0.0001\right)$. In particular, anemones colonized by $S$. minutum were significantly larger at week 8 than anemones colonized by any of the heterologous symbionts (Tukey post hoc, $P<0.0001$ for all comparisons; Fig. 4A), while anemones colonized by heterologous symbionts were no larger than aposymbiotic anemones (Tukey post hoc, $P>0.9$ for all comparisons). Anemones containing
S. minutum grew by an average of $9.22 \% \pm 0.68 \%$ between the beginning and the end of the experiment, while anemones containing $S$. trenchii and $S$. microadriaticum grew by just $1.9 \% \pm 1.06 \%$ and $1.54 \% \pm 1.05 \%$, respectively. Anemones containing $\mathrm{C} 3$ and $S$. voratum showed, on average, negative growth of $(-1.91 \%) \pm 0.65 \%$ and $(-1.71 \%) \pm 1.08 \%$, respectively. By comparison, aposymbiotic anemones grew $0.28 \% \pm$ $0.88 \%$.

Consistent with the differences in size and growth, after 8 weeks anemones colonized by $S$. minutum had produced significantly more pedal lacerates than did anemones colo-

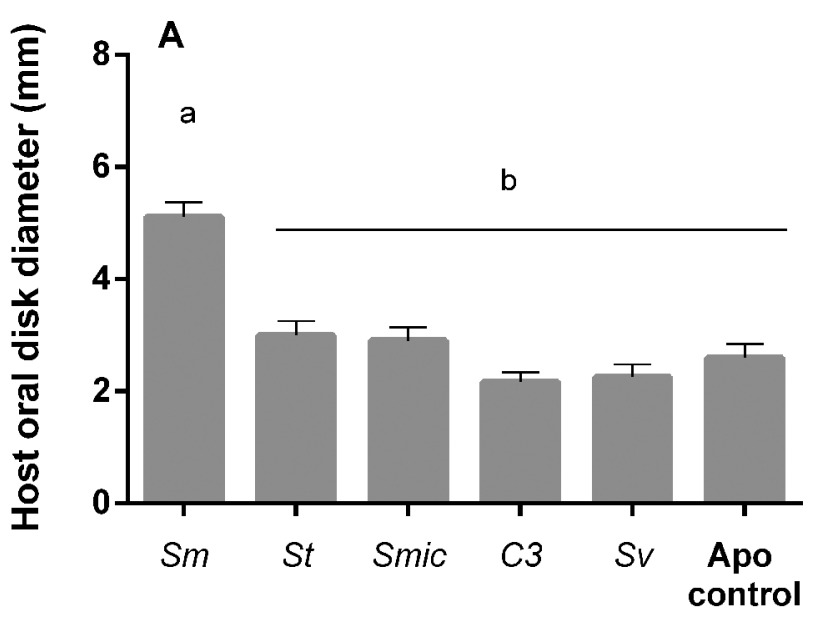

Symbiodinium species

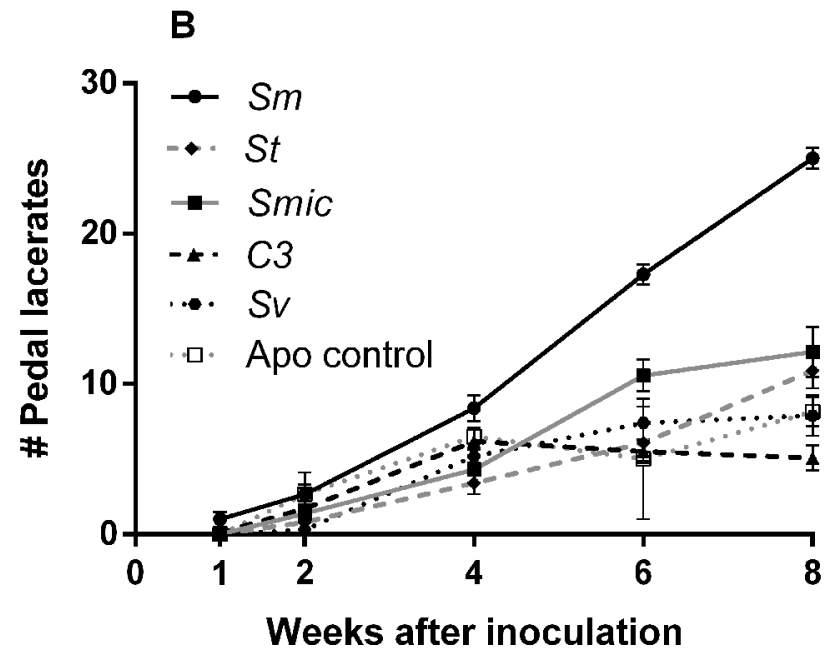

Figure 4. Host performance varied with symbiont identity. (A) Host growth estimated from the change in oral disk diameter (mm), quantified 8 weeks after colonization ( $n=10$ for each treatment). (B) Asexual reproduction via pedal laceration measured by counting pedal lacerates quantified over 8 weeks after inoculation $(n=10$ anemones per treatment and time point). Values are means \pm SE. Apo, aposymbiotic; C3, Symbiodinium phylotype C3; Sm, S. minutum; Smic, S. microadriaticum; St, S. trenchii; Sv, S. voratum. 
nized by the other symbionts $\left(\chi^{2}\right.$, Bonferroni post hoc, $P<$ 0.0001 for all comparisons; Fig. 4B). Symbiodinium minutumcolonized anemones produced an average of $25 \pm 0.58$ pedal lacerates per anemone between the start of the experiment and week 8 , compared to $12 \pm 1.48,11 \pm 1.20,7 \pm 1.11$, and $5 \pm 0.85$ pedal lacerates when in symbiosis with $S$. microadriaticum, S. trenchii, S. voratum, and type C3, respectively. By week $8, S$. microadriaticum-colonized anemones produced a number of pedal lacerates similar to that produced by $S$. trenchii-colonized anemones, and both produced a higher number of pedal lacerates than anemones inoculated with $S$. voratum or type C3 $\left(\chi^{2}\right.$, Bonferroni post hoc, $\left.P<0.05\right)$. Aposymbiotic anemones produced $8 \pm 0.76$ pedal lacerates per anemone by week 8 , a number similar to that produced by anemones inoculated with $S$. microadriaticum, S. trenchii, or $S$. voratum ( $\chi^{2}$, Bonferroni post hoc, $P>0.05$ for all comparisons) but a significantly higher number than for anemones inoculated with $\mathrm{C} 3\left(\chi^{2}\right.$, Bonferroni post hoc, $\left.P<0.05\right)$.

\section{Discussion}

\section{Colonization success varies with symbiont identity}

Colonization success varied with symbiont identity, with the homologous Symbiodinium minutum being fastest to proliferate and invade host tissues. Consistent with our results, previous colonization studies have shown that symbiotic algae are more immediately successful when originally isolated from the same host species (Kinzie and Chee, 1979; Schoenberg and

q11 Trench, 1980; Davy et al., 1997; Belda-Baillie et al., 2002; Harii et al., 2009; Starzak et al., 2014). For example, Weis et al. (2001) showed that homologous symbionts were able to colonize nearly all Fungia scutaria larvae inoculated and reach higher densities inside each larva than were heterologous symbionts, while some of these heterologous symbionts could not colonize the larvae at all.

In contrast, some of the other symbionts in our study failed or struggled to form a symbiosis with Aiptasia. In particular, C3 was taken up by the host, but there was no evidence of symbiont proliferation, while $S$. voratum did not persist inside the host beyond four weeks. Previous studies have likewise shown differential success of heterologous symbionts in Aiptasia. For example, Belda-Baillie et al. (2002) reported that adult Aiptasia could form only a limited symbiosis with cultured Symbiodinium A and was unable to form a symbiosis with a C-type and a free-living A-type, even after three months. Furthermore, S. voratum has previously been reported to be incapable of forming a symbiosis with both larval and adult Aiptasia, a finding that is consistent with our observations here (Xiang et al., 2013; Wolfowicz et al., 2016). Our results are, however, somewhat different from those of Starzak et al. (2014), who reported that $S$. voratum (culture ID CCMP421) was, on occasion, able to proliferate rapidly and reach high densities inside Aiptasia. The reason for the difference be- tween this study and the current study is unclear-not least given that the same cultures of Symbiodinium (CCMP421) and Aiptasia (NZ1) were used in both studies-though it is important to note that Starzak and coworkers also reported a relatively low percentage of colonization success and a high incidence of host mortality when anemones were inoculated with $S$. voratum.

The failure of Symbiodinium C3 and S. voratum to proliferate and persist in Aiptasia, respectively, despite uptake from the external environment, highlights the role of post-phagocytotic events in the establishment of a successful symbiosis, including the initiation of a functional molecular cross-talk between the host and the symbiont. Exactly what this molecular crosstalk involves remains unclear, though it is likely that molecular signals and/or nutritional interplay have a role in the evasion of host immunity by the invading symbiont and the prevention of symbiont loss via the likes of host expulsion or host-cell apoptosis (reviewed by Davy et al., 2012). The failure of $S$. voratum to persist is not surprising given its freeliving origin, as well as the fact that this species is relatively large compared to other symbiont species. We did not measure cell size here; however, a recent paper by Biquand et al. (2017) suggests that the colonization success of Symbiodinium in Aiptasia is negatively correlated with symbiont cell size. Our results, where different symbiont species or types had very different fates in symbiosis with Aiptasia, provide a baseline for further study of the molecular signals and events that dictate symbiosis success in this important model system.

While there were marked differences between the colonization success of the different symbionts, the spatial patterns of colonization were very similar, with algae first appearing in the oral disk before spreading to the tentacles, then the column, and, finally, the pedal disk. This pattern is perhaps not surprising given that the oral disk is likely the first point of contact between host and symbiont. The patchy pattern of symbiont proliferation in host tentacles (Fig. 2A) suggests that algal clusters arise from mitotic division of a single invading cell, with the algal symbionts moving either through the anemone's tissue or via transport in the gastrovascular cavity. Symbionts have been shown to pass between host cellular compartments during host oogenesis or in larvae in a range of cnidarians (Benayahu et al., 1992; Benayahu and Schleyer, 1998; Hirose et al., 2000, 2001; Davy and Turner, 2003; Marlow and Martindale, 2007); however, the putative migration distances to generate the patterns seen in our study, even in small adult Aiptasia, would be much greater, and, hence, this mechanism of proliferation seems implausible here. It is more likely that the symbionts exit host cells, move through the gastrovascular cavity via ciliary water currents, and invade other host cells elsewhere in the animal. While as a result of the technical limitations of our confocal method we could not confirm the presence of symbiont cells in the gastrovascular cavity of the tentacles, Parrin et al. $(2012,2016)$ observed within-colony symbiont migration in soft corals as a response to thermal stress, 
providing support for this proposed mechanism. This topic warrants detailed investigation.

\section{Host photophysiology and performance}

Host photophysiology and performance were clearly influenced by Symbiodinium identity, consistent with previous observations of differential photosynthetic performance in different Symbiodinium types (Rowan, 2004; Goulet et al., 2005; Berkelmans and van Oppen, 2006; Loram et al., 2007; Cantin et al., 2009). The homologous $S$. minutum was the most productive at the whole-symbiosis level and most beneficial with respect to host growth and pedal laceration, consistent with the carbon flux model of Starzak et al. (2014) for this same hostsymbiont combination. In contrast, $S$. microadriaticum and $S$. trenchii successfully colonized Aiptasia, reaching relatively high population densities (though not as high as $S$. minutum), but did not contribute substantially to host growth and proliferation. As expected given the inverse relationship between symbiont density and $\mathrm{CO}_{2}$ availability for photosynthesis (Davy and Cook, 2001), the lower density of these two heterologous symbionts relative to the homologous $S$. minutum elevated their photosynthetic rates per cell (data not shown); but this partial (with S. microadriaticum) or total (with S. trenchii) offset of total symbiosis photosynthesis was insufficient to generate comparable rates of host growth and asexual reproduction. In the case of $S$. microadriaticum, the low symbiont density may partially explain the relatively low photosynthetic rate of anemones colonized by this species compared to that of those colonized by $S$. minutum. However, the situation in the $S$. trenchii-colonized anemones was different, as their photosynthetic rate was similar to that of the $S$. minutum-colonized anemones. It therefore seems likely that $S$. trenchii released less of its photosynthate to the host, consistent with previous observations for some members of clade D (Cantin et al., 2009; Jones and Berkelmans, 2010). A further possibility is that $S$. trenchii is more energetically costly to maintain than $S$. minutum, though this is not supported by the respiration rates measured in our study, which were unaffected by symbiont identity. Previous evidence that some heterologous Symbiodinium taxa are energetically costly when in symbiosis with Aiptasia comes from Starzak et al. (2014), who found that $S$. voratum and type F5.1 induced high rates of host respiration that countered any photosynthetic benefits and ultimately led to a low P: R ratio and little scope for host growth and reproduction; these authors did not study the impacts of S. trenchii, however. The disadvantages of harboring some members of Symbiodinium clade D on host fitness have been reported previously. For example, when adult and juvenile Acropora sp. associated with Symbiodinium from clade D, it exhibited a decrease in lipid storage, egg size, and overall colony growth, compared to when it hosted Symbiodinium C2/1 (Little et al., 2004; Jones and Berkelmans, 2011).
The behavior of $S$. microadriaticum and $S$. trenchii is interesting because it is consistent with the cheater strategy exhibited by many parasites, as well as strains of the typically mutualistic Rhizobium in plants, where colonization is not associated with any discernible benefit for the host (Herre et al., 1999; Sachs and Simms, 2006; Sachs et al., 2010; Sachs et al., 2011). Previous studies have similarly suggested that several members of Symbiodinium clades A and D are less beneficial than those of, for example, clades B and C (Cantin et al., 2009; Mieog et al., 2009; Jones and Berkelmans, 2010; Stat and Gates, 2011; Lesser et al., 2013). How these symbiont species persisted in Aiptasia is currently unknown, but they might somehow evade the host's immune responses and regulatory pathways and draw reserves from the host to support their metabolism and growth while providing little or no photosynthetically fixed carbon to the host in return. Further research on the differential regulation of these symbiont populations and the exchange of metabolites between the partners will help shed light on this matter. Ultimately, this information will enable us to better understand the long-term implications of harboring more versus less beneficial symbionts, especially in terms of the ability of coral reefs to recover from bleaching events and adapt as our climate warms.

\section{Acknowledgments}

This work was supported by a Victoria University of Wellington postgraduate scholarship to YG. This project was funded by the Marsden Fund of the Royal Society of New Zealand, grant 1202, to SKD and VMW. We thank Dr. Mark Warner and another anonymous reviewer for their helpful comments.

\section{Literature Cited}

Baker, A. C. 2003. Flexibility and specificity in coral-algal symbiosis: diversity, ecology, and biogeography of Symbiodinium. Annu. Rev. Ecol. Evol. Syst. 34: 661-689.

Baumgarten, S., O. Simakov, L. Y. Esherick, Y. J. Liew, E. M. Lehnert, C. T. Michell, Y. Li, E. A. Hambleton, A. Guse, M. E. Oates et al. 2015. The genome of Aiptasia, a sea anemone model for coral symbiosis. Proc. Natl. Acad. Sci. U.S.A. 112: 11893-11898.

Belda-Baillie, C. A., B. K. Baillie, and T. Maruyama. 2002. Specificity of a model cnidarian-dinoflagellate symbiosis. Biol. Bull. 202: 74-85.

Benayahu, Y., and M. H. Schleyer. 1998. Reproduction in Anthelia glauca (Octocorallia: Xeniidae). I. Gametogenesis and larval brooding. Mar. Biol. 131: 423-432.

Benayahu, Y., D. Weil, and Z. Malik. 1992. Entry of algal symbionts into oocytes of the coral Litophyton arboreum. Tissue Cell 24: 473-482.

Berkelmans, R., and M. J. H. van Oppen. 2006. The role of zooxanthellae in the thermal tolerance of corals: a "nugget of hope" for coral reefs in an era of climate change. Proc. R. Soc. Biol. Sci. B 273: 2305-2312.

Biquand, E., N. Okubo, Y. Aihara, V. Rolland, D. C. Hayward, M. Hatta, J. Minagawa, T. Maruyama, and S. Takahashi. 2017. Acceptable symbiont cell size differs among cnidarian species and may limit symbiont diversity. ISME J. 11: 1702-1712.

Bradford, M. M. 1976. A rapid and sensitive method for the quantitation of microgram quantities of protein utilizing the principle of protein-dye binding. Anal. Biochem. 72: 248-254. 
Cantin, N. E., M. J. H. van Oppen, B. L. Willis, J. C. Mieog, and A. P. Negri. 2009. Juvenile corals can acquire more carbon from highperformance algal symbiosis. Coral Reefs 28: 405-414.

Chang, S. S., B. B. Prézelin, and R. K. Trench. 1983. Mechanisms of photoadaptation in three strains of the symbiotic dinoflagellate Symbiodinium microadriaticum. Mar. Biol. 76: 219-229.

Coffroth, M. A., D. M. Poland, E. L. Petrou, D. A. Brazeau, and J. C. Holmberg. 2010. Environmental symbiont acquisition may not be the solution to warming seas for reef-building corals. PLoS One 5: e13258.

Davy, S., and C. Cook. 2001. The relationship between nutritional status and carbon flux in the zooxanthellate sea anemone Aiptasia pallida. Mar. Biol. 139: 999-1005.

Davy, S. K., and J. R. Turner. 2003. Early development and acquisition of zooxanthellae in the temperate symbiotic sea anemone Anthopleura ballii (Cocks). Biol. Bull. 205: 66-72.

Davy, S. K., I. A. N. Lucas, and J. R. Turner. 1997. Uptake and persistence of homologous and heterologous zooxanthellae in the temperate sea anemone Cereus pedunculatus (Pennant). Biol. Bull. 192: 208-216.

Davy, S. K., D. Allemand, and V. M. Weis. 2012. Cell biology of cnidariandinoflagellate symbiosis. Microbiol. Mol. Biol. Rev. 76: 229-261.

Fitt, W. K., S. S. Chang, and R. K. Trench. 1981. Motility patterns of different strains of the symbiotic dinoflagellate Symbiodinium (= Gymnodinium) microadriaticum (Freudenthal) in culture. Bull. Mar. Sci. 31: 436443.

Goulet, T. L., C. B. Cook, and D. Goulet. 2005. Effect of short-term exposure to elevated temperatures and light levels on photosynthesis of different host-symbiont combinations in the Aiptasia pallida-Symbiodinium symbiosis. Limnol. Oceanogr. 50: 1490-1498.

Grajales, A., and E. Rodríguez. 2014. Morphological revision of the genus Aiptasia and the family Aiptasiidae (Cnidaria, Actiniaria, Metridioidea). Zootaxa 3826: 55-100.

Harii, S., N. Yasuda, M. Rodriguez-Lanetty, T. Irie, and M. Hidaka. 2009. Onset of symbiosis and distribution patterns of symbiotic dinoflagellates in the larvae of scleractinian corals. Mar. Biol. 156: 12031212.

Hennige, S. J., D. J. Suggett, M. E. Warner, K. E. McDougall, and D. J. Smith. 2009. Photobiology of Symbiodinium revisited: bio-physical and bio-optical signatures. Coral Reefs 28: 179-195.

Herre, E. A., N. Knowlton, U. G. Mueller, and S. A. Rehner. 1999. The evolution of mutualisms: exploring the paths between conflict and cooperation. Trends Ecol. Evol. 14: 49-53.

Hirose, M., R. A. Kinzie III, and M. Hidaka. 2000. Early development of zooxanthella-containing eggs of the corals Pocillopora verrucosa and $P$. eydouxi with special reference to the distribution of zooxanthellae. Biol. Bull. 199: 68-75.

Hirose, M., R. Kinzie, and M. Hidaka. 2001. Timing and process of entry of zooxanthellae into oocytes of hermatypic corals. Coral Reefs 20: 273-280.

Iglesias-Prieto, R., V. H. Beltran, T. C. LaJeunesse, H. Reyes-Bonilla, and P. E. Thome. 2004. Different algal symbionts explain the vertical distribution of dominant reef corals in the eastern Pacific. Proc. R. Soc. Biol. Sci. B 271: 1757-1763.

Jeong, H. J., Y. Du Yoo, N. S. Kang, A. S. Lim, K. A. Seong, S. Y. Lee, M. J. Lee, K. H. Lee, H. S. Kim, W. Shin et al. 2012. Heterotrophic feeding as a newly identified survival strategy of the dinoflagellate Symbiodinium. Proc. Natl. Acad. Sci. U.S.A. 109: 12604-12609.

Jones, A., and R. Berkelmans. 2010. Potential costs of acclimatization to a warmer climate: growth of a reef coral with heat tolerant vs. sensitive symbiont types. PLoS One 5: e10437.

Jones, A. M., and R. Berkelmans. 2011. Tradeoffs to thermal acclimation: energetics and reproduction of a reef coral with heat tolerant Symbiodinium type-D. J. Mar. Biol. 2011: 1-12.

Kinzie, R. A., III, and G. S. Chee. 1979. The effect of different zooxanthellae on the growth of experimentally reinfected hosts. Biol. Bull. 156: 315-327.
LaJeunesse, T. C. 2002. Diversity and community structure of symbiotic dinoflagellates from Caribbean coral reefs. Mar. Biol. 141: 387-400.

LaJeunesse, T. C., D. J. Thornhill, E. F. Cox, F. G. Stanton, W. K. Fitt, and G. W. Schmidt. 2004. High diversity and host specificity observed among symbiotic dinoflagellates in reef coral communities from Hawaii. Coral Reefs 23: 596-603.

Leal, M. C., K. Hoadley, D. T. Pettay, A. Grajales, R. Calado, and M. E. Warner. 2015. Symbiont type influences trophic plasticity of a model cnidarian-dinoflagellate symbiosis. J. Exp. Biol. 218: 858-863.

Lesser, M. P., M. Stat, and R. D. Gates. 2013. The endosymbiotic dinoflagellates (Symbiodinium sp.) of corals are parasites and mutualists. Coral Reefs 32: 603-611.

Little, A. F., M. J. H. van Oppen, and B. L. Willis. 2004. Flexibility in algal endosymbioses shapes growth in reef corals. Science 304: 14921494.

Loram, J. E., H. G. Trapido-Rosenthal, and A. E. Douglas. 2007. Functional significance of genetically different symbiotic algae Symbiodinium in a coral reef symbiosis. Mol. Ecol. 16: 4849-4857.

Marlow, H. Q., and M. Q. Martindale. 2007. Embryonic development in two species of scleractinian coral embryos: Symbiodinium localization and mode of gastrulation. Evol. Dev. 9: 355-367.

Matthews, J. L., A. E. Sproles, C. A. Oakley, A. R. Grossman, V. M. Weis, and S. K. Davy. 2015. Menthol-induced bleaching rapidly and effectively provides experimental aposymbiotic sea anemones (Aiptasia sp.) for symbiosis investigations. J. Exp. Biol. 219: 306-310.

Mieog, J. C., J. L. Olsen, R. Berkelmans, S. A. Bleuler-Martinez, B. L. Willis, and M. J. H. van Oppen. 2009. The roles and interactions of symbiont, host and environment in defining coral fitness. PLoS One 4: e6364.

Parrin, A. P., K. L. Harmata, S. E. Netherton, M. A. Yaeger, L. S. Bross, and N. W. Blackstone. 2012. Within-colony migration of symbionts during bleaching of octocorals. Biol. Bull. 223: 245-256.

Parrin, A. P., T. L. Goulet, M. A. Yaeger, L. S. Bross, C. S. McFadden, and N. W. Blackstone. 2016. Symbiodinium migration mitigates bleaching in three octocoral species. J. Exp. Mar. Biol. Ecol. 474: 73-80.

Pettay, D. T., and T. C. Lajeunesse. 2009. Microsatellite loci for assessing genetic diversity, dispersal and clonality of coral symbionts in "stresstolerant" clade D Symbiodinium. Mol. Ecol. Resour. 9: 1022-1025.

Pettay, D. T., D. C. Wham, R. T. Smith, R. Iglesias-Prieto, and T. C. LaJeunesse. 2015. Microbial invasion of the Caribbean by an IndoPacific coral zooxanthella. Proc. Natl. Acad. Sci. U.S.A. 112: 7513-7518.

Pochon, X., and R. D. Gates. 2010. A new Symbiodinium clade (Dinophyceae) from soritid foraminifera in Hawai'i. Mol. Phylogenet. Evol. 56: $492-497$.

Robison, J. D., and M. E. Warner. 2006. Differential impacts of photoacclimation and thermal stress on the photobiology of four different phylotypes of Symbiodinium (Pyrrhophyta). J. Phycol. 42: 568-579.

Roth, M. S. 2014. The engine of the reef: photobiology of the coral-algal symbiosis. Front. Microbiol. 5: 422.

Rowan, R. 2004. Coral bleaching: thermal adaptation in reef coral symbionts. Nature 430: 742.

Sachs, J. L., and E. L. Simms. 2006. Pathways to mutualism breakdown. Trends Ecol. Evol. 21: 585-592.

Sachs, J. L., C. J. Essenberg, and M. M. Turcotte. 2011. New paradigms for the evolution of beneficial infections. Trends Ecol. Evol. 26: 202-209.

Schoenberg, D. A., and R. K. Trench. 1980a. Genetic variation in Symbiodinium (= Gymnodinium) microadriaticum Freudenthal, and specificity in its symbiosis with marine invertebrates. I. Isoenzyme and soluble protein patterns of axenic cultures of Symbiodinium microadriaticum. Proc. R. Soc. Biol. Sci. B 207: 405-427.

Schoenberg, D. A., and R. K. Trench. 1980b. Genetic variation in Symbiodinium (= Gymnodinium) microadriaticum Freudenthal, and specificity in its symbiosis with marine invertebrates. III. Specificity and infectiv- 
ity of Symbiodinium microadriaticum. Proc. R. Soc. Biol. Sci. B 207: 445460.

Silverstein, R. N., R. Cunning, and A. C. Baker. 2015. Change in algal symbiont communities after bleaching, not prior heat exposure, increases heat tolerance of reef corals. Glob. Chang. Biol. 21: 236-249.

Starzak, D. E., R. G. Quinnell, M. R. Nitschke, and S. K. Davy. 2014. The influence of symbiont type on photosynthetic carbon flux in a model cnidarian-dinoflagellate symbiosis. Mar. Biol. 161: 711-724.

Stat, M., and R. D. Gates. 2011. Clade D Symbiodinium in scleractinian corals: a "nugget" of hope, a selfish opportunist, an ominous sign, or all of the above? J. Mar. Biol. 2011: 1-9.

q15 Stat, M., C. E. Bird, X. Pochon, L. Chasqui, L. J. Chauka, G. T. Concepcion, D. Logan, M. Takabayashi, R. J. Toonen, and R. D. Gates. 2011. Variation in Symbiodinium ITS 2 sequence assemblages among coral colonies. PLoS One 6: e15854.

Wang, J.-T., Y.-Y. Chen, K. S. Tew, P.-J. Meng, and C. A. Chen. 2012. Physiological and biochemical performances of menthol-induced aposymbiotic corals. PLoS One 7: e46406.
Weis, V. M., W. S. Reynolds, M. D. deBoer, and D. A. Krupp. 2001. Host-symbiont specificity during onset of symbiosis between the dinoflagellates Symbiodinium spp. and planula larvae of the scleractinian coral Fungia scutaria. Coral Reefs 20: 301-308.

Weis, V. M., S. K. Davy, O. Hoegh-Guldberg, M. Rodriguez-Lanetty, and J. R. Pringle. 2008. Cell biology in model systems as the key to understanding corals. Trends Ecol. Evol. 23: 369-376.

Wolfowicz, I., S. Baumgarten, P. A. Voss, E. A. Hambleton, C. R. Voolstra, M. Hatta, and A. Guse. 2016. Aiptasia sp. larvae as a model to reveal mechanisms of symbiont selection in cnidarians. Sci. Rep. 6: 32366.

Xiang, T., E. A. Hambleton, J. C. Denofrio, J. R. Pringle, and A. R. Grossman. 2013. Isolation of clonal axenic strains of the symbiotic dinoflagellate Symbiodinium and their growth and host specificity. J. Phycol. 49: 447-458.

Yuyama, I., T. Nakamura, T. Higuchi, and M. Hidaka. 2016. Different stress tolerances of juveniles of the coral Acropora tenuis associated with clades C1 and D Symbiodinium. Zool. Stud. 55: 1-9. 


\section{Appendix 2}

\section{ITS2 sequences of Symbiodinium taxa used in this study}

Symbiodinium taxa ( $\mathrm{n}=3$ samples per culture or isolate) used in experiments in Chapters 2, 3,4 and 5 were genetically identified prior to inoculation of Aiptasia and at the end of the experiments, using the isolation and molecular techniques described in the methods section of each chapter. All treatments were found to harbour the same Symbiodinium species as the one inoculated at the start of the experiment. Sequences of the 5.8s ribosomal RNA gene (partial sequence), internal transcribed spacer 2 (ITS2; complete sequence) and 28s ribosomal RNA gene (partial sequence) for Symbiodinium species/phylotypes used in this study are as follows: (A) $S$. minutum (ITS2 B1); (B) $S$. microadriaticum (ITS2 A1); (C) $S$. trenchii (ITS2 D1a); (D) Symbiodinium C3; and (E) S. voratum (ITS2 E). Sequences presented are derived from those provided by Macrogen Inc. confirming the consistency of the symbiont population pre- and post-inoculation.

(A)

$\begin{array}{cccccc}1 & 10 & 20 & 30 & 40 & 50 \\ \mid & \mid & \mid & \mid & \mid\end{array}$

GCTGCTTCAGTGCTTAGCATTATCTACCTGTGCTTGCAAGCAGCATGTAT

GTCTGCATTGCTGCTTCGCTTTCCAACAAGTCATCGATCGCTTTTGTGTT

CGTAAATGGCTTGTTTGCTGCCTGGCCCATGCGCCAAGCTTGAGCGTACT

GTTGTTCCAAGCTTTGCTTGCATCGTGCAGCTCAAGCGCGCAGCTGTCGG

GATGCTGATGCATGCCCTTAGCATGAAGTCAGACAAGAGAACCCGCTGAA

TTTAAGCATATAA 
(B)

$\begin{array}{cccccc}1 & 10 & 20 & 30 & 40 & 50 \\ \mid & \mid & \mid & \mid & \mid & \mid\end{array}$

ACGTGCTTGCGCTCTTGGGATTGCCTGAGAGCATGTCTGCTTCAGTGCTT CATACTTTCATTTTCTGCTGCTCTTGTTATCAGGAGCAGTGTTGCTGCATG CTTCTGCAAGTGGCACTGGCATGCTAAATATCAAGTTTTGCTTGCTGTTG TGACTGATCAACATCTCATGTCGTTTCAGTTGGCGAAACAAAAGCTCATG TGTGTTCTTAACACTTCCTAGCATGAAGTCAGACAAGTGAACCCGCTGAA TTTAAGCATATAAGTAA

(C)

$\begin{array}{rrrrrr}1 & 10 & 20 & 30 & 40 & 50 \\ \mid & \mid & \mid & \mid & \mid & \mid\end{array}$

ACGCGCTTGCCTCTTGGGACTTCCTGAGAGTATGTTTGCTTCAGTGCTTG TTTTACCTCCTTGCAAGGTTCTGTCGCAACCTTGTGCCCTGGCCAGCCAC GGGTTAACTTGCCCATGGCTTGCTGAGTAGTGATCTTTTAGAGCAAGCTC TGGCACGCTGTTGTTTGAGGCAGCCTATATTGAGGCTATTTCAAATGACG TTGCTACAAGCTTGATGTGTCCTTCTGCGCCGTTGCGCATCCCATAGCAT GAAGTCAAACAAGAGAACCCGCTGAACTTAAGCATATGGATCC

(D)

$\begin{array}{cccccc}1 & 10 & 20 & 30 & 40 & 50 \\ \mid & \mid & \mid & \mid & \mid & \mid\end{array}$

ACGTACGTTGCACTCTTGGGATTTCCTGAGAGTATGTCTGCTTCAGTGCT TAGCTTGCCCAATCTTGCGGATAGACTTTGTTTCTGTCTTGCGCCCCTGT GAGCCATTGAGCGTCTAGTCAATGGCTTATTGAATGATTCGGTCTTGCAA AAGCTTTGCGCGCTTCTATTCAAGATTCCACCTTGGAGTGGTATTGCTTG AGTGACGCTGCTCATGCTTGCAACTGGGATGCTAACGCATGCCTCTAGC ATGAAGTCAGACAAGCGA 
(E)

$\begin{array}{cccccc}1 & 10 & 20 & 30 & 40 & 50 \\ \mid & \mid & \mid & \mid & \mid\end{array}$

TCTGCTTCAGTGCTTTTCATATCTTCGCAGTGCGGGCTTCCTGGAGAAGC CTTGAGCCTCTTTGCGCGCTGCTGCATCAGAATTTGCAGCGGCGCGCTGA ACACAAACCGGGAGGTAAGCTGGACTGATTTGTCGCGCATCACTGGGCAC GTGTGTCCGTTTTGGCCCAATCATGCCAGCCTGCCAAGCAATTGGTGCTC AAATACCAATCTTAGCATGAAGTCAGACAAGCAAACCCGCTGAATTTAAG CATATAA 


\section{Appendix 3}

\section{Calculation of ITS2 copies for Symbiodinium species using quantitative PCR}

Table A3.1. Number of ITS2 copies calculated for each Symbiodinium species using quantitative PCR. (A) $S$. microadriaticum versus $S$. minutum; (B) S. minutum versus $S$. trenchii. A $=$ S. microadriaticum, $\mathrm{B}=$ S. minutum and $\mathrm{D}=$ S. trenchii.

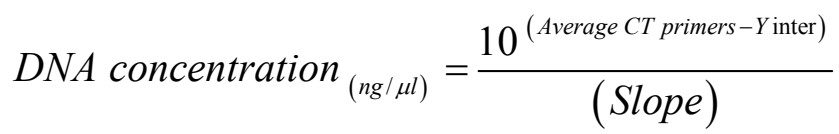

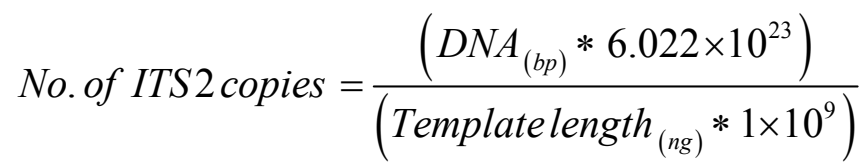

http://cels.uri.edu/gsc/cndna.html 
(A)

\begin{tabular}{|c|c|c|c|c|c|c|c|c|c|c|c|c|}
\hline \multirow[b]{2}{*}{$\mathrm{A}: \mathrm{A}+\mathrm{B}$} & \multirow{2}{*}{$\begin{array}{l}\text { Cell counts } \\
\text { Cells per ml }\end{array}$} & \multirow[b]{2}{*}{$\begin{array}{c}\text { Vol A } \\
\text { extracted } \\
(\mathrm{ml})\end{array}$} & \multirow[b]{2}{*}{$\begin{array}{c}\text { No. A } \\
\text { cells } \\
\text { extracted }\end{array}$} & \multirow[b]{2}{*}{$\begin{array}{c}\text { No. A } \\
\text { cells per } \\
\mu \mathrm{l} \\
\text { template }\end{array}$} & \multirow[b]{2}{*}{$\begin{array}{c}\text { No. ITS2 } \\
\text { copies } \\
\text { per A cell }\end{array}$} & \multicolumn{3}{|c|}{ From qPCR } & \multirow[b]{2}{*}{$\begin{array}{c}\text { Primer A } \\
\text { average } \\
\text { CT }\end{array}$} & \multirow[b]{2}{*}{$\begin{array}{c}\text { Type A } \\
\text { concentration } \\
(\mathrm{ng} / \mathrm{ul})\end{array}$} & \multirow[b]{2}{*}{$\begin{array}{c}\text { Type A } \\
\text { copies per } \\
\mu 1\end{array}$} & \multirow[b]{2}{*}{$\begin{array}{c}\text { Type A } \\
\text { cells per } \mu 1\end{array}$} \\
\hline & & & & & & $\begin{array}{c}\text { Primer A } \\
\text { replicate } \\
1\end{array}$ & $\begin{array}{l}\text { Primer A } \\
\text { replicate } \\
2\end{array}$ & $\begin{array}{c}\text { Primer A } \\
\text { replicate } \\
3\end{array}$ & & & & \\
\hline 0.99 & 9350250 & 0.099 & 925674.8 & 18513.495 & 5.3 & 19.959 & 19.765 & 19.897 & 19.874 & 0.0005946 & 98105.073 & 18510.391 \\
\hline 0.9 & 9350250 & 0.09 & 841522.5 & 16830.45 & 5.3 & 20.131 & 19.965 & 20.120 & 20.072 & 0.0005213 & 86016.609 & 16229.549 \\
\hline 0.8 & 9350250 & 0.08 & 748020 & 14960.4 & 5.3 & 20.382 & 20.311 & 20.383 & 20.359 & 0.0004309 & 71102.772 & 13415.617 \\
\hline 0.5 & 9350250 & 0.05 & 467512.5 & 9350.25 & 5.3 & 21.169 & 21.063 & 20.967 & 21.066 & 0.0002694 & 44445.081 & 8385.864 \\
\hline 0.2 & 9350250 & 0.02 & 187005 & 3740.1 & 5.3 & 23.044 & 22.421 & 22.631 & 22.699 & $9.108 \mathrm{E}-05$ & 15028.671 & 2835.598 \\
\hline 0.1 & 9350250 & 0.01 & 93502.5 & 1870.05 & 5.3 & 23.666 & 23.771 & 24.811 & 24.083 & $3.632 \mathrm{E}-05$ & 5993.520 & 1130.853 \\
\hline 0.01 & 9350250 & 0.001 & 9350.25 & 187.005 & 5.3 & 27.578 & 26.963 & 27.321 & 27.287 & $4.324 \mathrm{E}-06$ & 713.507 & 134.624 \\
\hline
\end{tabular}

\begin{tabular}{|c|c|c|c|c|c|c|c|c|c|c|c|}
\hline \multirow[b]{2}{*}{$\begin{array}{c}\text { Vol B } \\
\text { extracted } \\
(\mathrm{ml})\end{array}$} & \multirow[b]{2}{*}{$\begin{array}{c}\text { No. B } \\
\text { cells } \\
\text { extracted }\end{array}$} & \multirow[b]{2}{*}{$\begin{array}{c}\text { No. B } \\
\text { cells per } \\
\mu \mathrm{l} \\
\text { template }\end{array}$} & \multirow[b]{2}{*}{$\begin{array}{c}\text { No. ITS2 } \\
\text { copies } \\
\text { per B cell }\end{array}$} & \multicolumn{3}{|c|}{ From qPCR } & \multirow[b]{2}{*}{$\begin{array}{c}\text { Primer B } \\
\text { average } \\
\text { CT }\end{array}$} & \multirow[b]{2}{*}{$\begin{array}{c}\text { Type B } \\
\text { concentration } \\
(\mathrm{ng} / \mu \mathrm{l})\end{array}$} & \multirow[b]{2}{*}{$\begin{array}{c}\text { Type B } \\
\text { copies per } \\
\mu \mathrm{l}\end{array}$} & \multirow[b]{2}{*}{$\begin{array}{c}\text { Type B } \\
\text { cells per } \mu 1\end{array}$} & \multirow[b]{2}{*}{$\begin{array}{r}\text { Predictec } \\
\text { A:A+B }\end{array}$} \\
\hline & & & & $\begin{array}{c}\text { Primer B } \\
\text { replicate } \\
1\end{array}$ & $\begin{array}{c}\text { Primer B } \\
\text { replicate } \\
2 \\
\end{array}$ & $\begin{array}{c}\text { Primer B } \\
\text { replicate } \\
3\end{array}$ & & & & & \\
\hline 0.001 & 9350.25 & 187.005 & 1.65 & 27.679 & 27.600 & 27.708 & 27.662 & $2.454 \mathrm{E}-06$ & 402.419 & 243.890 & 0.987 \\
\hline 0.01 & 93502.5 & 1870.05 & 1.65 & 24.616 & 24.384 & 24.467 & 24.489 & $2.227 \mathrm{E}-05$ & 3652.364 & 2213.554 & 0.880 \\
\hline 0.02 & 187005 & 3740.1 & 1.65 & 23.599 & 23.671 & 23.558 & 23.610 & 4.104E-05 & 6730.110 & 4078.854 & 0.767 \\
\hline 0.05 & 467512.5 & 9350.25 & 1.65 & 22.304 & 22.434 & 22.249 & 22.329 & $9.994 \mathrm{E}-05$ & 16389.382 & 9932.959 & 0.458 \\
\hline 0.08 & 748020 & 14960.4 & 1.65 & 21.736 & 21.817 & 22.960 & 22.171 & 0.0001115 & 18290.896 & 11085.392 & 0.204 \\
\hline 0.09 & 841522.5 & 16830.45 & 1.65 & 21.639 & 21.518 & 21.506 & 21.554 & 0.0001712 & 28075.101 & 17015.212 & 0.062 \\
\hline 0.099 & 925674.8 & 18513.495 & 1.65 & 21.199 & 21.303 & 21.249 & 21.250 & 0.0002115 & 34681.526 & 21019.107 & 0.006 \\
\hline
\end{tabular}


(B)

\begin{tabular}{|c|c|c|c|c|c|c|c|c|c|c|c|c|}
\hline \multirow[b]{2}{*}{$B: B+D$} & \multirow{2}{*}{$\begin{array}{l}\text { Cell counts } \\
\\
\begin{array}{c}\text { Cells per } \\
\text { ml }\end{array} \\
\end{array}$} & \multirow[b]{2}{*}{$\begin{array}{l}\text { Vol B } \\
\text { extracted } \\
(\mathrm{ml})\end{array}$} & \multirow[b]{2}{*}{$\begin{array}{c}\text { No. B } \\
\text { cells } \\
\text { extracted }\end{array}$} & \multirow[b]{2}{*}{$\begin{array}{c}\text { No. B cells } \\
\text { per } \mu 1 \\
\text { template }\end{array}$} & \multirow[b]{2}{*}{$\begin{array}{c}\text { No. ITS2 } \\
\text { copies } \\
\text { per B } \\
\text { cell } \\
\end{array}$} & \multicolumn{3}{|c|}{ From qPCR } & \multirow[b]{2}{*}{$\begin{array}{c}\text { Primer B } \\
\text { average } \\
\text { CT }\end{array}$} & \multirow[b]{2}{*}{$\begin{array}{c}\text { Type B } \\
\text { concentration } \\
(\mathrm{ng} / \mu \mathrm{l})\end{array}$} & \multirow[b]{2}{*}{$\begin{array}{c}\text { Type B } \\
\text { copies per } \mu 1\end{array}$} & \multirow[b]{2}{*}{$\begin{array}{c}\text { Type B } \\
\text { cells per } \mu 1 \\
\end{array}$} \\
\hline & & & & & & $\begin{array}{c}\text { Primer B } \\
\text { replicate } \\
1\end{array}$ & $\begin{array}{l}\text { Primer B } \\
\text { replicate } \\
2 \\
\end{array}$ & $\begin{array}{c}\text { Primer B } \\
\text { replicate } \\
3 \\
\end{array}$ & & & & \\
\hline 0.99 & 9350250 & 0.099 & 925674.8 & 18513.495 & 1.65 & 23.189 & 22.638 & 21.902 & 22.577 & $8.413 \mathrm{E}-05$ & 13797.714 & 8362.251 \\
\hline 0.9 & 9350250 & 0.09 & 841522.5 & 16830.45 & 1.65 & 22.318 & 22.273 & 21.721 & 22.104 & 0.0001168 & 19161.478 & 11613.017 \\
\hline 0.8 & 9350250 & 0.08 & 748020 & 14960.4 & 1.65 & 22.544 & 22.448 & 21.973 & 22.322 & 0.0001005 & 16474.007 & 9984.247 \\
\hline 0.5 & 9350250 & 0.05 & 467512.5 & 9350.25 & 1.65 & 23.459 & 23.373 & 23.422 & 23.418 & 4.689E-05 & 7689.729 & 4660.442 \\
\hline 0.2 & 9350250 & 0.02 & 187005 & 3740.1 & 1.65 & 24.520 & 23.824 & 23.817 & 24.054 & $3.013 \mathrm{E}-05$ & 4942.126 & 2995.228 \\
\hline 0.1 & 9350250 & 0.01 & 93502.5 & 1870.05 & 1.65 & 25.344 & 24.903 & 24.806 & 25.018 & $1.542 \mathrm{E}-05$ & 2529.375 & 1532.955 \\
\hline 0.01 & 9350250 & 0.001 & 9350.25 & 187.005 & 1.65 & 27.711 & 28.634 & 28.649 & 28.331 & $1.542 \mathrm{E}-06$ & 252.839 & 153.236 \\
\hline
\end{tabular}

\begin{tabular}{|c|c|c|c|c|c|c|c|c|c|c|c|}
\hline \multirow[b]{2}{*}{$\begin{array}{l}\text { Vol D } \\
\text { extracted } \\
(\mathrm{ml})\end{array}$} & \multirow[b]{2}{*}{$\begin{array}{c}\text { No. D } \\
\text { cells } \\
\text { extracted }\end{array}$} & \multirow[b]{2}{*}{$\begin{array}{c}\text { No. D cells } \\
\text { per } \mu \text { l } \\
\text { template }\end{array}$} & \multirow[b]{2}{*}{$\begin{array}{c}\text { No. ITS2 } \\
\text { copies } \\
\text { per D } \\
\text { cell }\end{array}$} & \multicolumn{3}{|c|}{ From qPCR } & \multirow[b]{2}{*}{$\begin{array}{l}\text { Primer D } \\
\text { average } \\
\text { CT }\end{array}$} & \multirow[b]{2}{*}{$\begin{array}{c}\text { Type D } \\
\text { concentration } \\
(\mathrm{ng} / \mathrm{ul})\end{array}$} & \multirow[b]{2}{*}{$\begin{array}{c}\text { Type D } \\
\text { copies per } \mu 1\end{array}$} & \multirow[b]{2}{*}{$\begin{array}{c}\text { Type D } \\
\text { cells per } \mu 1\end{array}$} & \multirow[b]{2}{*}{$\begin{array}{c}\text { Predictec } \\
\text { B:B+D }\end{array}$} \\
\hline & & & & $\begin{array}{c}\text { Primer D } \\
\text { replicate } \\
1\end{array}$ & $\begin{array}{c}\text { Primer D } \\
\text { replicate } \\
2\end{array}$ & $\begin{array}{c}\text { Primer D } \\
\text { replicate } \\
3\end{array}$ & & & & & \\
\hline 0.001 & 9350.25 & 187.005 & 212 & 22.172 & 22.226 & 22.333 & 22.244 & 0.000143 & 24597.880 & 116.028 & 0.986 \\
\hline 0.01 & 93502.5 & 1870.05 & 212 & 18.421 & 18.394 & 18.580 & 18.465 & 0.0018302 & 314788.211 & 1484.850 & 0.887 \\
\hline 0.02 & 187005 & 3740.1 & 212 & 17.215 & 17.382 & 17.973 & 17.524 & 0.0034545 & 594166.521 & 2802.672 & 0.781 \\
\hline 0.05 & 467512.5 & 9350.25 & 212 & 15.801 & 16.109 & 16.206 & 16.038 & 0.0094082 & 1618204.400 & 7633.040 & 0.379 \\
\hline 0.08 & 748020 & 14960.4 & 212 & 14.955 & 15.137 & 15.179 & 15.091 & 0.0178325 & 3067193.782 & 14467.895 & 0.172 \\
\hline 0.09 & 841522.5 & 16830.45 & 212 & 14.667 & 14.977 & 14.985 & 14.876 & 0.020604 & 3543889.779 & 16716.461 & 0.084 \\
\hline 0.099 & 925674.8 & 18513.495 & 212 & 14.510 & 14.856 & 14.912 & 14.759 & 0.0223021 & 3835965.566 & 18094.177 & 0.008 \\
\hline
\end{tabular}



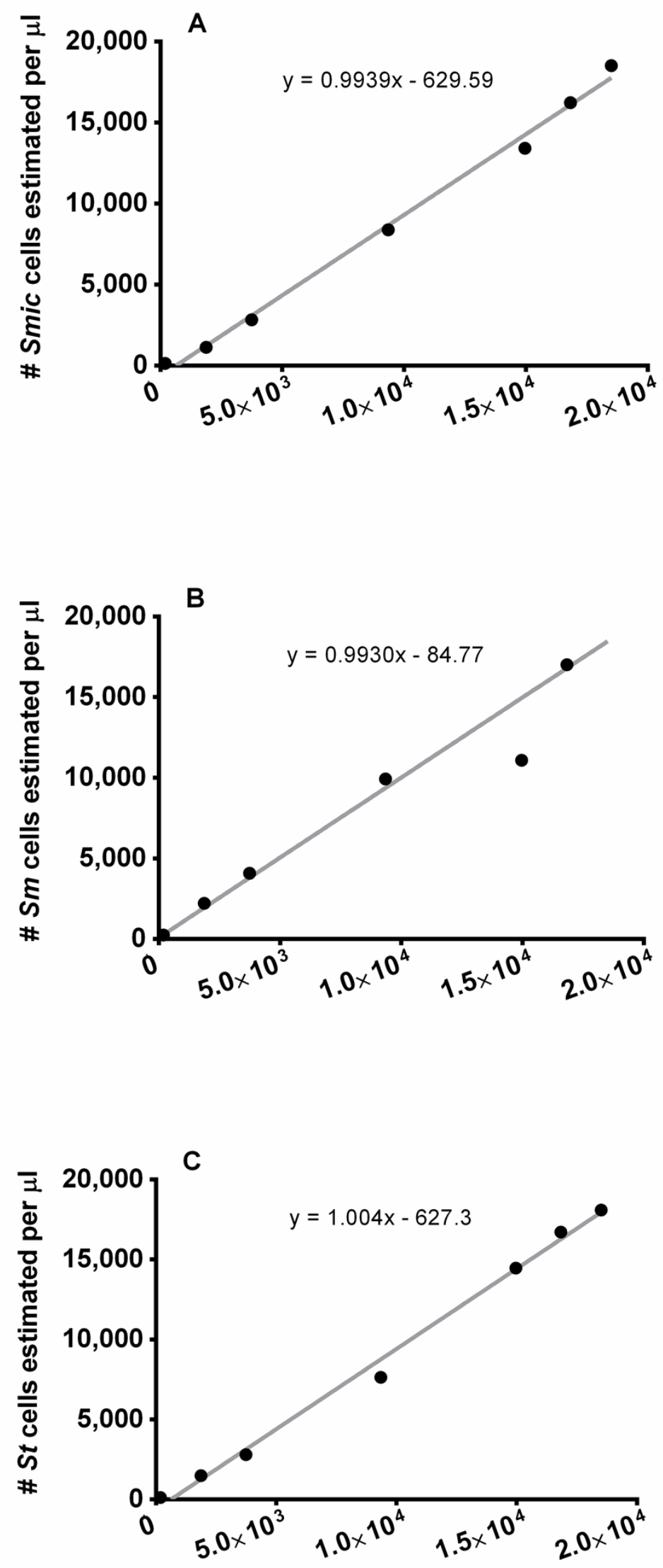

Cells counted per $\mu$ l template
Fig. A3.1. Quantitative PCR standard curves with 5 serial $\log 10$ dilutions of $1-10,000 \mathrm{ng} \cdot$ $\mu 1^{-1} \quad$ DNA: Counted symbiont cells per $\mu 1$ template versus estimated symbiont cells per $\mu 1$, calculated from quantitative PCR (Table A2.1). (A) $S$. microadriaticum; (B) S. minutum; and (C) S. trenchii. Smic $=S$. microadriaticum, $S m=S$. minutum, $S t=S$. trenchii . 


\section{Appendix 4}

\section{Simultaneous competitive exposure in a model cnidarian- dinoflagellate symbiosis under heat stress}
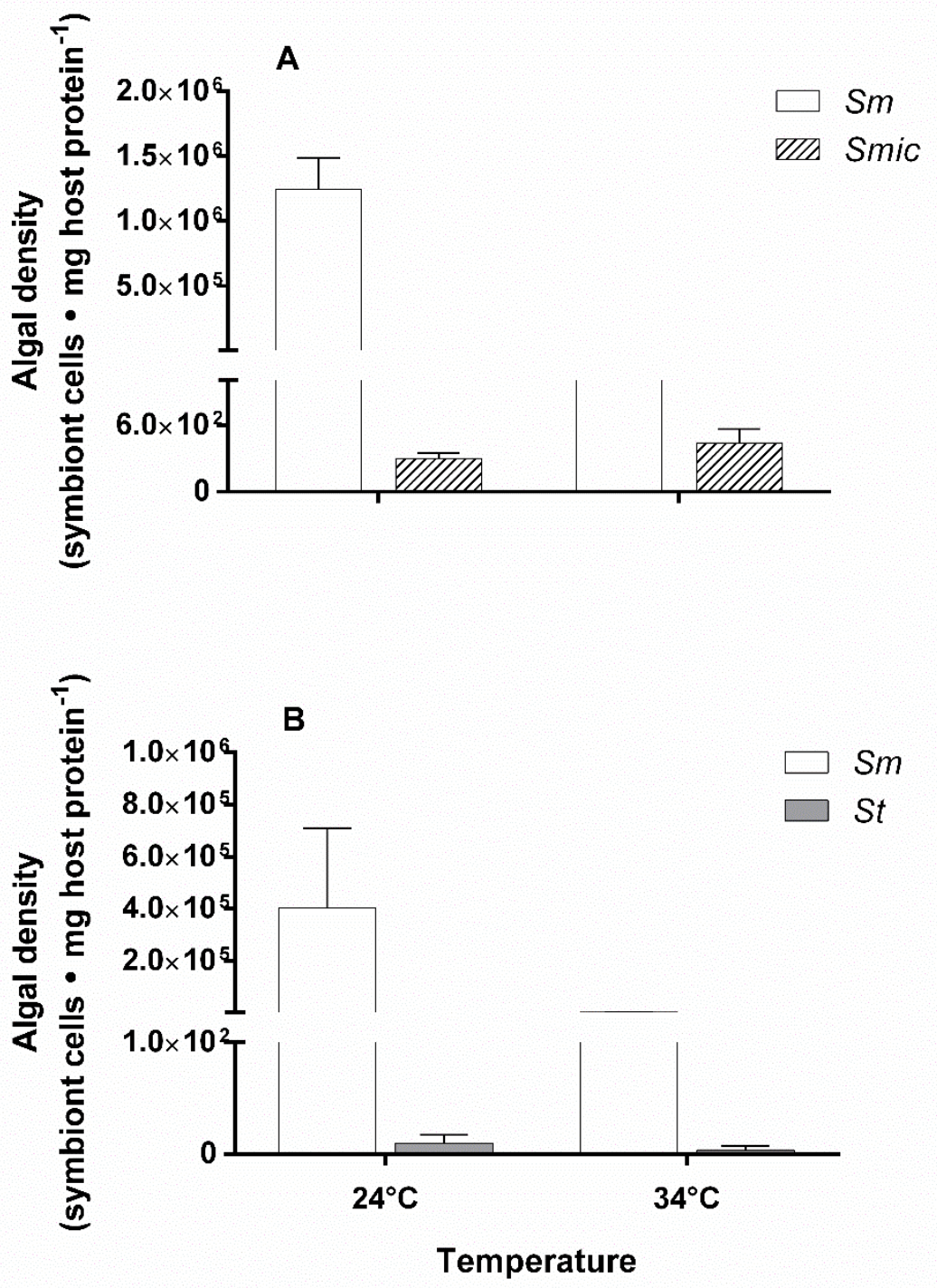

Fig. A4.1. Thermal bleaching in Aiptasia containing different mixtures of symbiont species. Symbiont density (symbiont cells $\cdot \mathrm{mg}$ host protein ${ }^{-1}$ ) in host anemones was quantified four weeks after inoculation for both control $\left(24{ }^{\circ} \mathrm{C}\right)$ and thermal stress $\left(34{ }^{\circ} \mathrm{C}\right)$ conditions (n $=7$ anemones per treatment). (A) $S m+S m i c$; (B) $S m+S t . S m=S$. minutum; Smic $=S$. microadriaticum $;$ St $=S$. trenchii. Bars represent means \pm SE. 


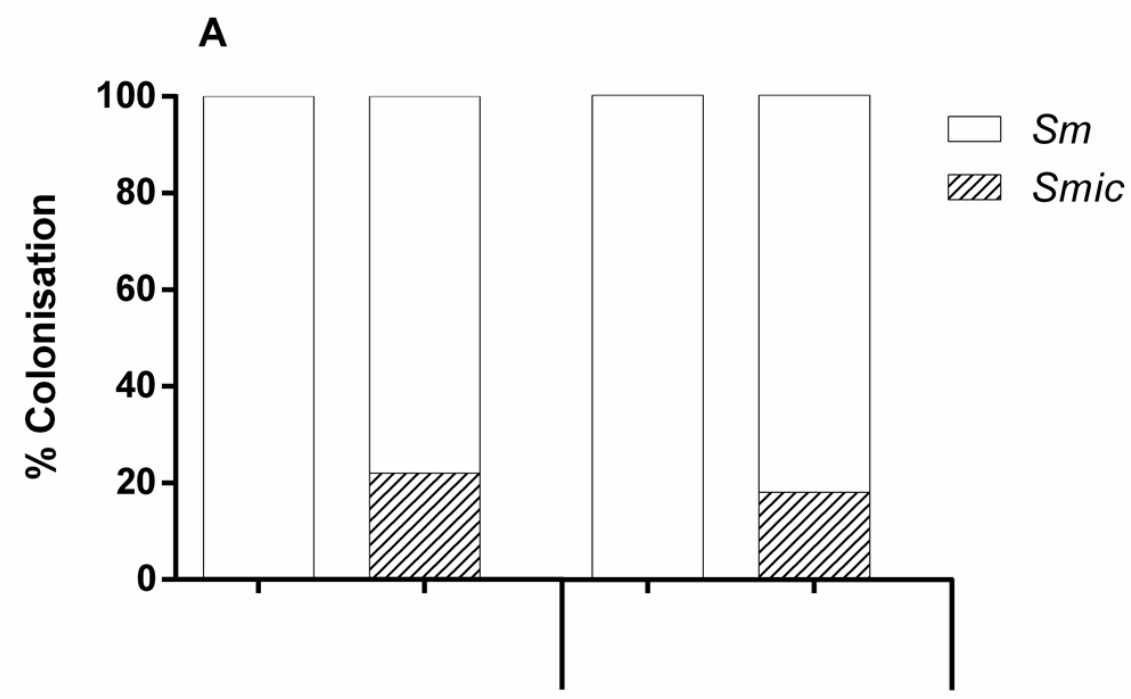

B

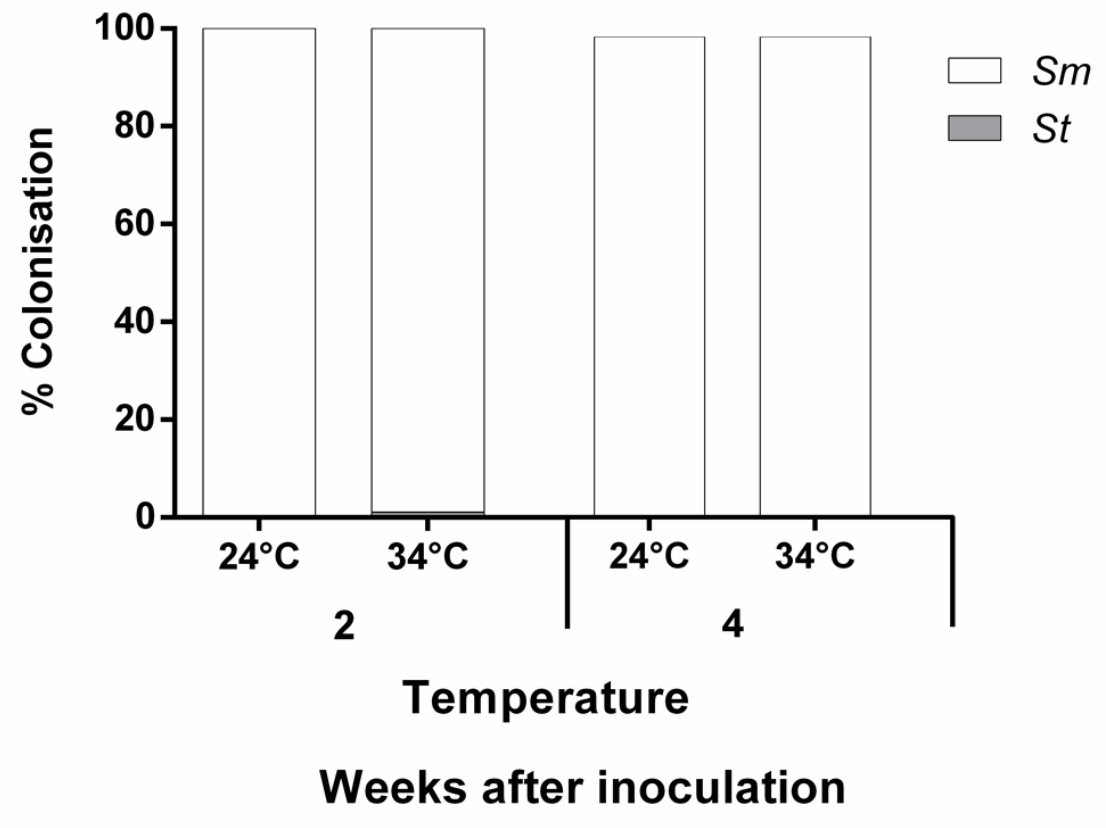

Fig. A4.2. Symbiont success in mixed populations varied with Symbiodinium species and temperature. Percentage colonisation success of the different Symbiodinium species when simultaneously inoculated into Aiptasia was quantified two and four weeks after inoculation under both control $\left(24^{\circ} \mathrm{C}\right)$ and thermal stress $\left(34^{\circ} \mathrm{C}\right)$ conditions $(\mathrm{n}=7$ anemones per treatment per time point). (A) $S m+S m i c$ (B) $S m+S t . S m=S$. minutum; Smic $=S$. microadriaticum; $S t=S$. trenchii. 


\section{Appendix 5}

\section{Measuring $F_{\mathrm{v}} / F_{m}$ as a proxy for a proxy for algal health in hospite under thermal stress}

\section{A5.1. Materials and methods}

Aposymbiotic Aiptasia were generated as described elsewhere. Following one week of starvation, a total of 80 aposymbiotic anemones of similar size (2-3 mm oral disc diameter) were transferred to $400 \mathrm{ml}$ clear plastic jars (one anemone per jar) filled with FSW, and allowed to settle for three days. Cultured Symbiodinium from three clades were used as inoculates: the homologous species $S$. minutum, and the heterologous species $S$. microadriaticum and $S$. trenchii (see Table 2.1 for details). Each algal culture was diluted in $25 \mathrm{ml} \mathrm{FSW}$ and one drop of Artemia nauplii suspension added, to give a final concentration of $\sim 1 \times 10^{6}$ cells $/ \mathrm{ml}$. The anemones were inoculated with one of the three different symbiont cultures ( $n=20$ anemones for each Symbiodinium species; $n=20$ uninoculated anemones as a negative control), by pipetting $1 \mathrm{ml}$ of this dinoflagellate suspension onto the oral disc of each anemone with a glass pipette. The inoculated polyps were maintained at $24{ }^{\circ} \mathrm{C}$ until they became densely colonised (four weeks). Anemones were then slowly acclimated to higher temperatures (control 25, 30, 32 and $34{ }^{\circ} \mathrm{C} ; 5$ anemones per algal species per temperature). Algal health was then monitored using a Pulse Amplitude Modulated (PAM) fluorometer (Diving PAM, Heinz Walz GmbH) over a period of three weeks, with sampling every morning (Fig. A5.1). During this time, the anemones were fed twice weekly with Artemia nauplii, with each feeding followed by a water-change. Irradiance was held at $\sim 100 \mu \mathrm{mol}$ photons $\bullet \mathrm{m}^{-2} \cdot \mathrm{s}^{-1}$ on a 12 -h light/12-h dark cycle. 
A

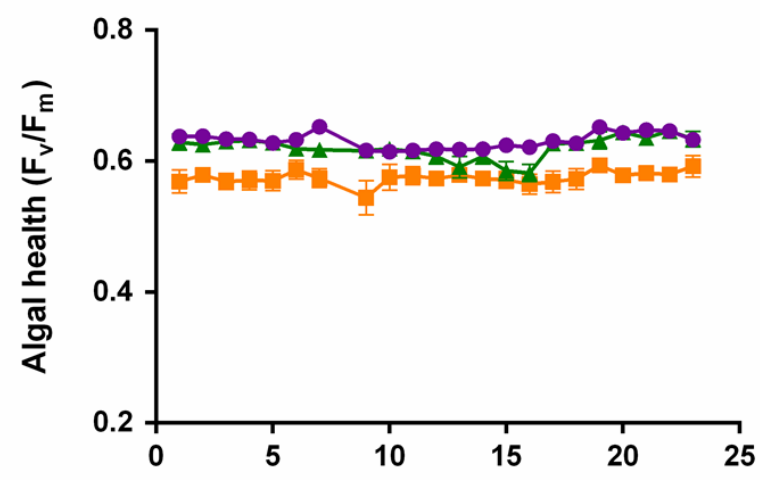

B

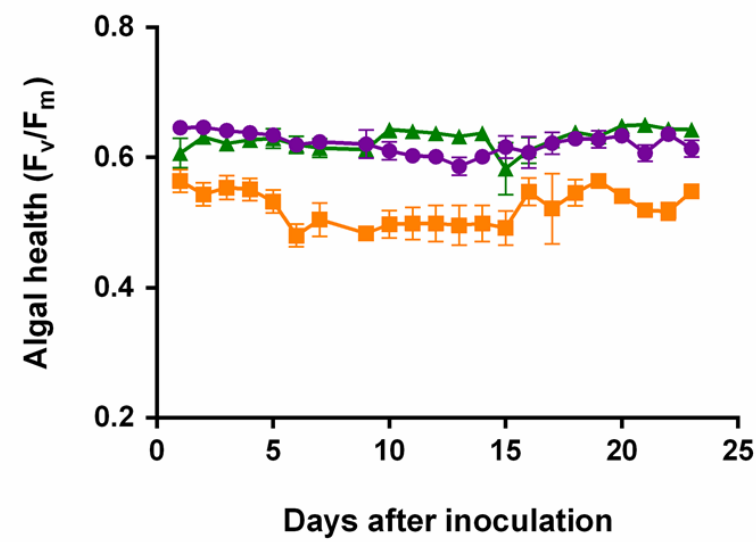

C

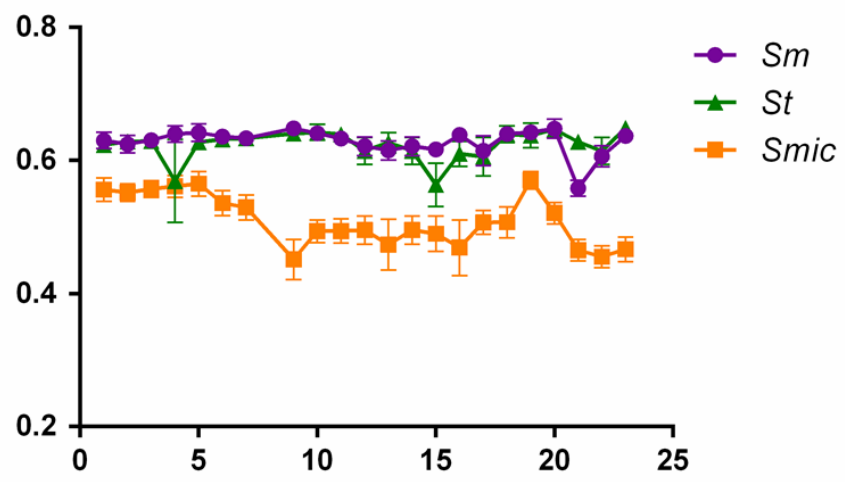

D

$34{ }^{\circ} \mathrm{C}$

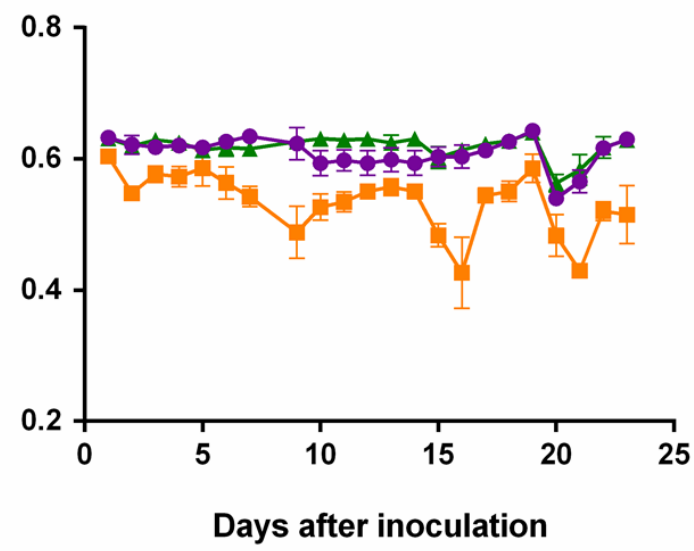

Fig. A5.1. Fv/Fm as a proxy for algal health in hospite was quantified for 23 days after inoculation ( $\mathrm{n}=5$ anemones per treatment per time point). (A) Algae at $25^{\circ} \mathrm{C}$; (B) $30^{\circ} \mathrm{C}$; (C) $32{ }^{\circ}$ C; (D) $34{ }^{\circ} \mathrm{C} . S m=S$. minutum; Smic $=S$. microadriaticum; St $=S$. trenchii. Points are means $\pm \mathrm{SE}$. 


\section{Appendix 6}

\section{Flow cytometry analysis of cell cycle progression}
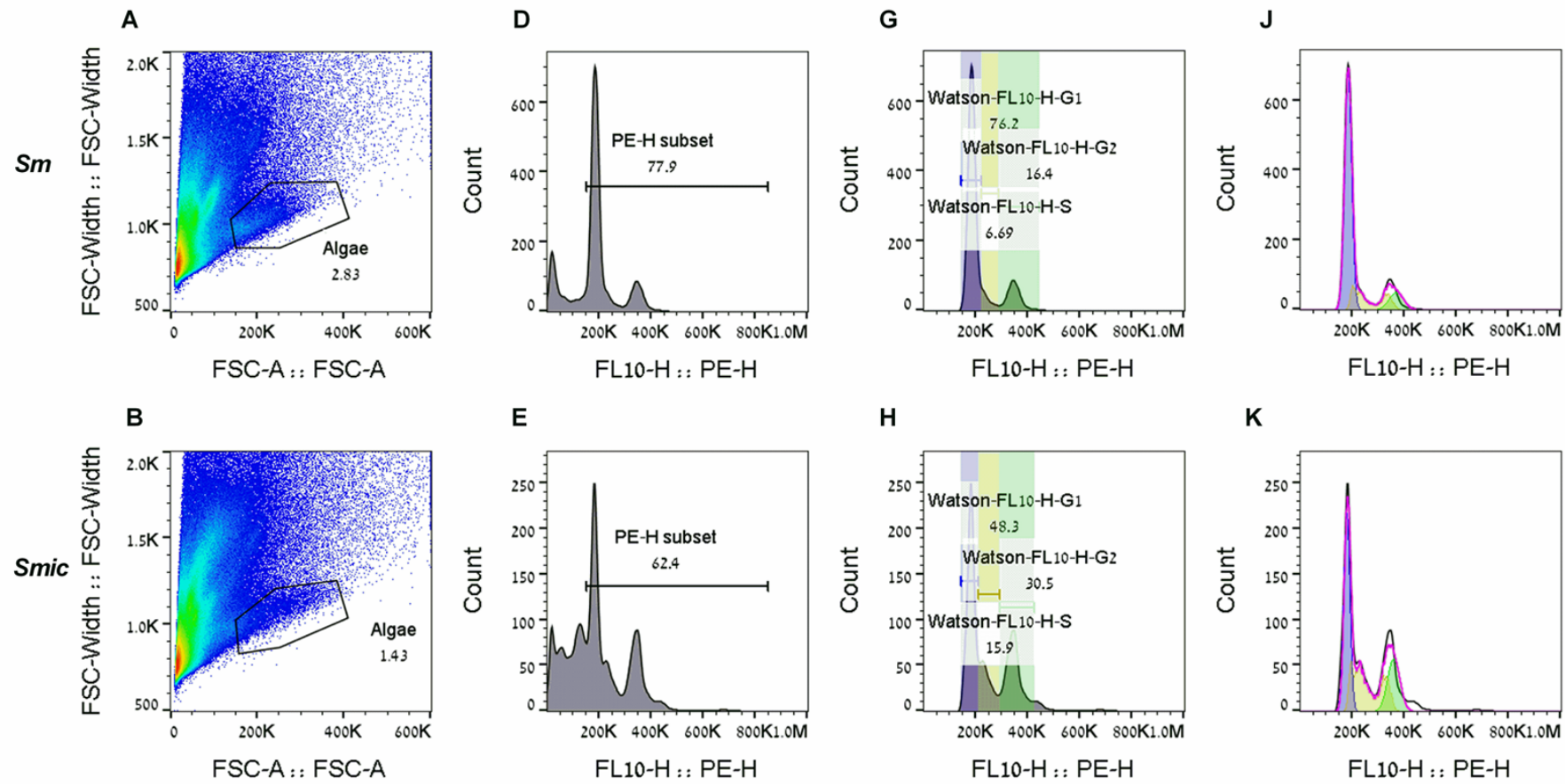

$$
\text { E }
$$

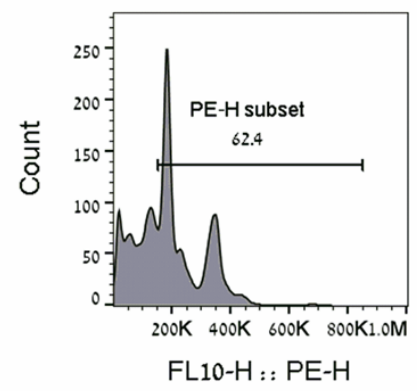

H
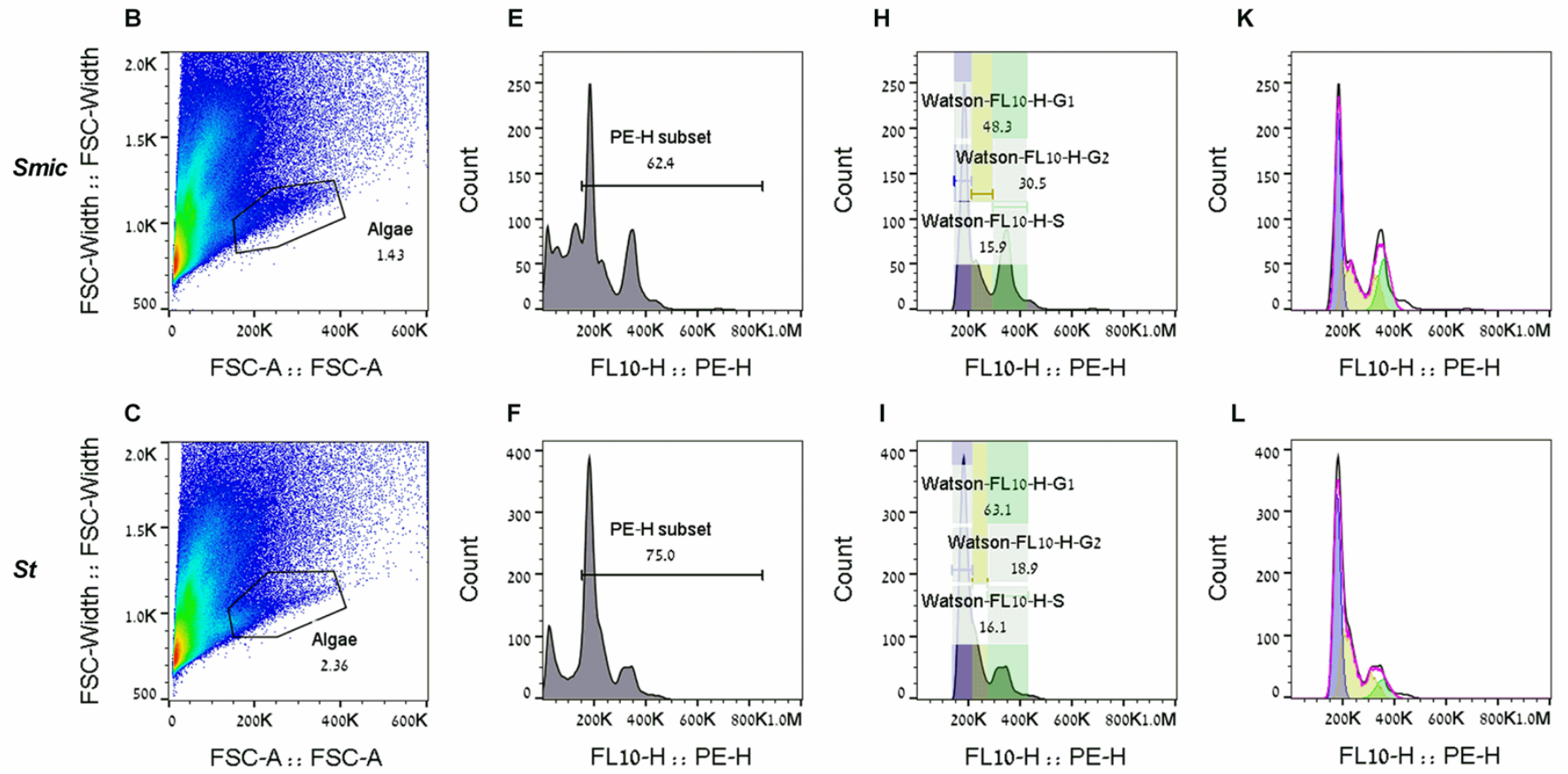

Fig. A6.1. Flow cytometry analysis of cell cycle progression of homologous and heterologous symbionts in Aiptasia. Cell cycle analysis of different symbiont species (rows: Symbiodinium minutum, S. microadriaticum, $S$. trenchii) one week after inoculation (n $=4$ anemones per sample; 8 samples per treatment). (A-C) Forward scatter count-area (FSC-A); (D-F) a subset of stained algae using the PE-H channel; (G-I) percentage of cells in different cell cycle phases $\left(\mathrm{G}_{1}, \mathrm{~S}, \mathrm{G}_{2}+\mathrm{M}\right)$; (J-L) graphic cell cycle of the algae. The algal signal was gated based on the controls (see Chapter 5, Fig. 5.1). Events recorded in the PE channel (DL) were restricted to events falling within the FSC gating window using FlowJo 10. $S m=S$. minutum, Smic $=S$. microadriaticum, $S t=S$. trenchii. 


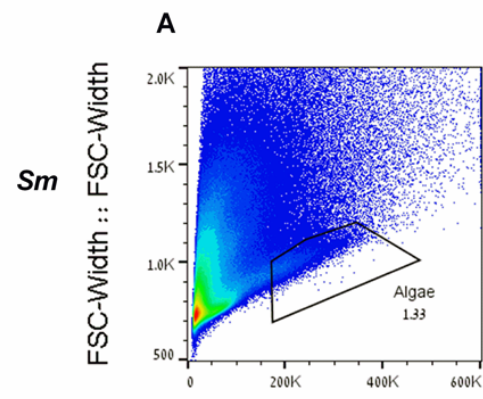

FSC-A : : FSC-A

B

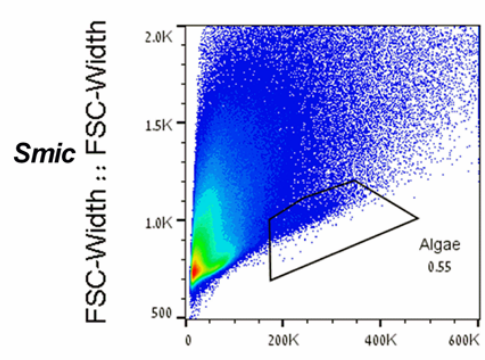

FSC-A : : FSC-A

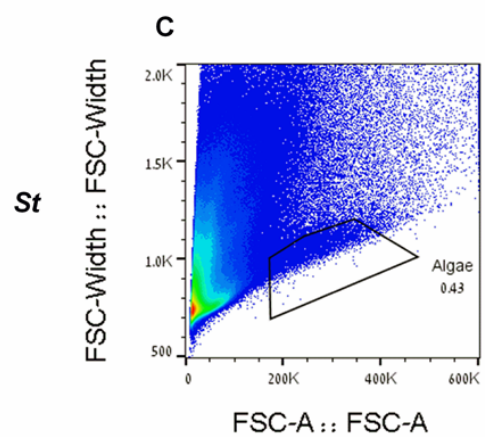

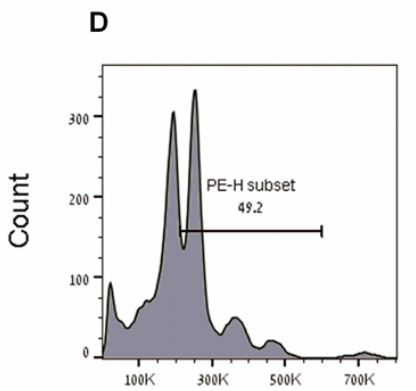

FL10-H : : PE-H

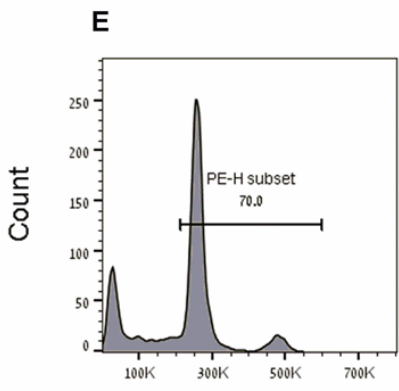

FL10-H : : PE-H

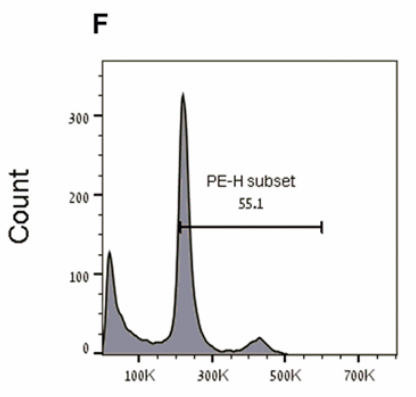

FL10-H : : PE-H

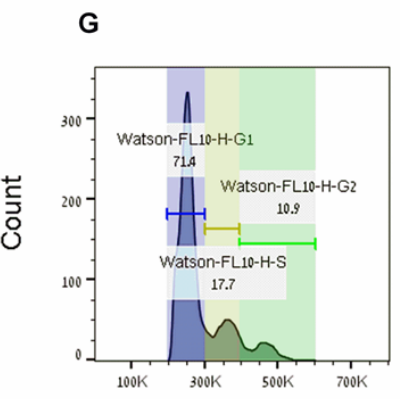

FL10-H : : PE-H

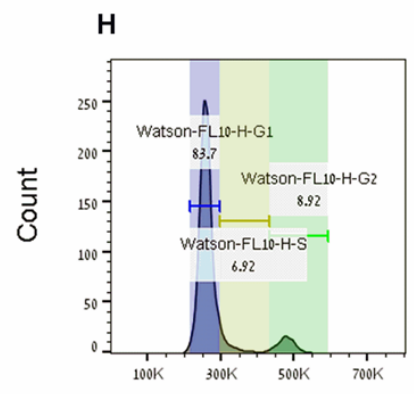

FL10-H : : PE-H

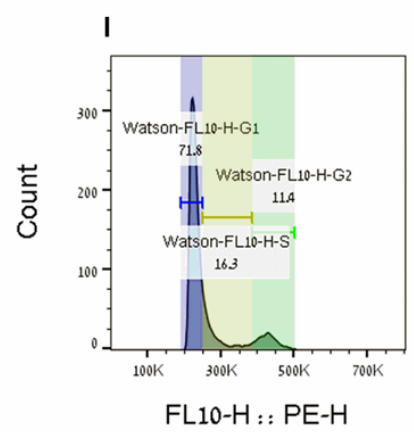

$\mathbf{J}$
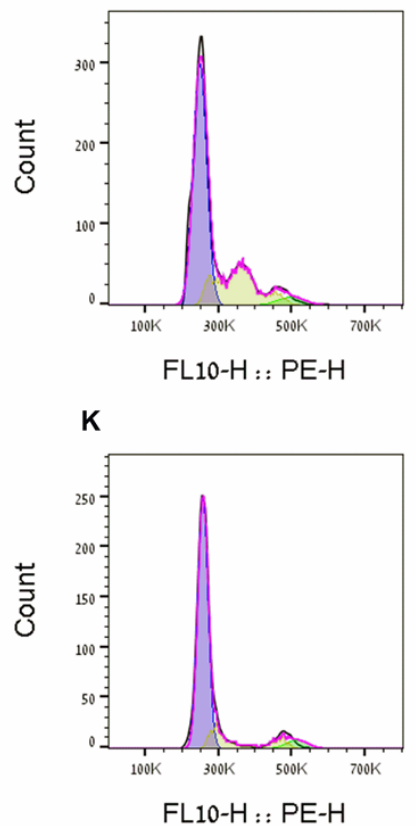

L

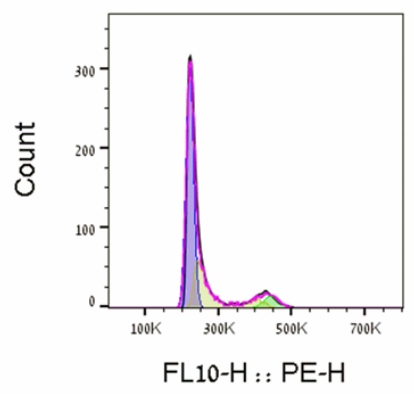

Fig. A6.2. Flow cytometry analysis of cell cycle progression of homologous and heterologous symbionts in Aiptasia. Cell cycle analysis of different symbiont species (rows: Symbiodinium minutum, S. microadriaticum, $S$. trenchii) two weeks after inoculation (n $=4$ anemones per sample; 8 samples per treatment). (A-C) Forward scatter count-area (FSC-A); (D-F) a subset of stained algae using the PE-H channel; (G-I) percentage of cells in different cell cycle phases $\left(\mathrm{G}_{1}, \mathrm{~S}, \mathrm{G}_{2}+\mathrm{M}\right)$; (J-L) graphic cell cycle of the algae. The algal signal was gated based on the controls (See Chapter 5, Fig. 5.1). Events recorded in the PE channel (DL) were restricted to events falling within the FSC gating window using FlowJo 10. $S m=S$. minutum, Smic $=S$. microadriaticum, $S t=S$. trenchii. 

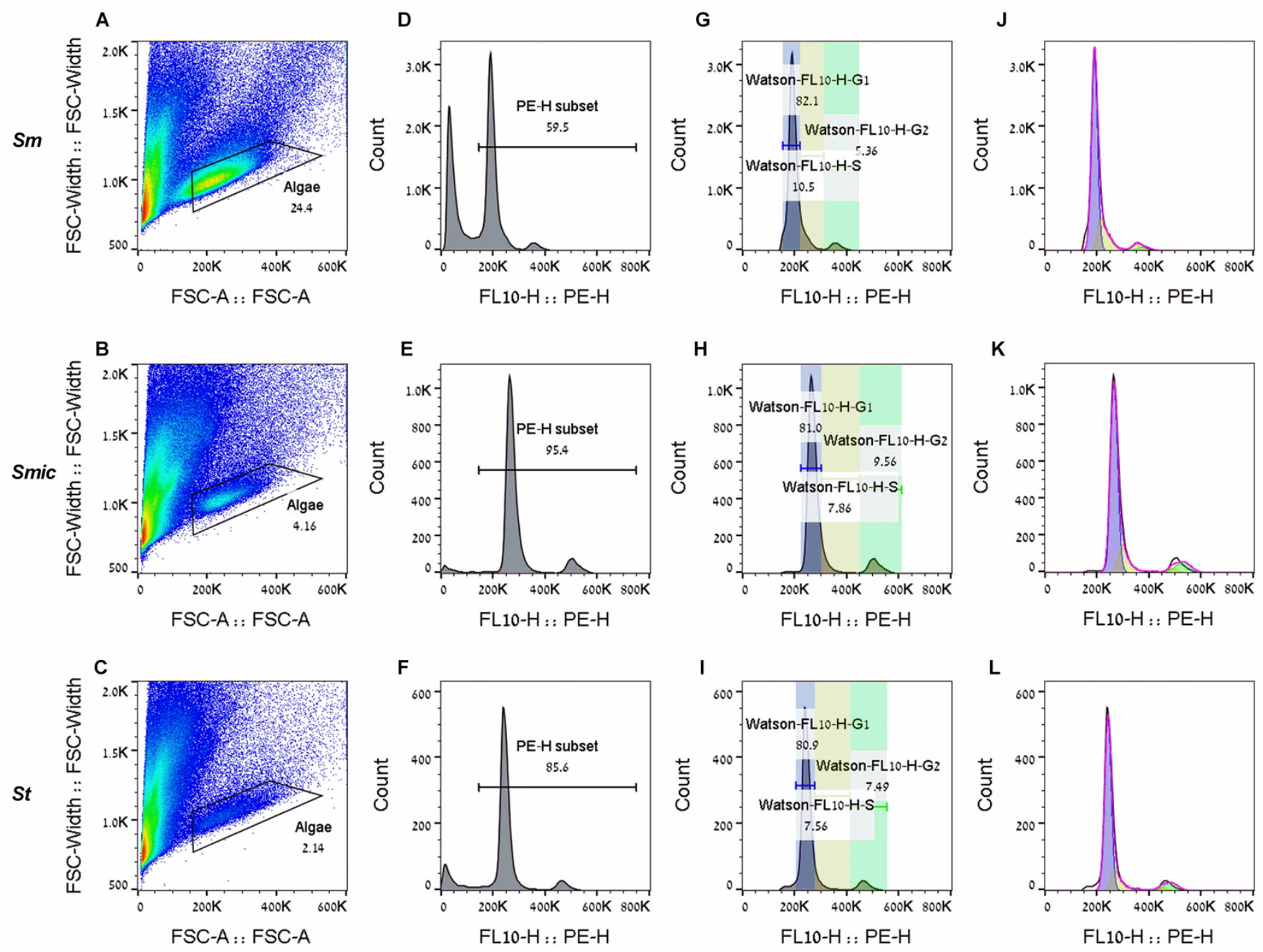

Fig. A6.3. Flow cytometry analysis of cell cycle progression of homologous and heterologous symbionts in Aiptasia. Cell cycle analysis of different symbiont species (rows: Symbiodinium minutum, $S$. microadriaticum, $S$. trenchii) four weeks after inoculation (n $=4$ anemones per sample; 8 samples per treatment). (A-C) Forward scatter count-area (FSC-A); (D-F) a subset of stained algae using the PE-H channel; (G-I) percentage of cells in different cell cycle phases $\left(\mathrm{G}_{1}, \mathrm{~S}, \mathrm{G}_{2}+\mathrm{M}\right)$; (J-L) graphic cell cycle of the algae. The algal signal was gated based on the controls (See Chapter 5, Fig. 5.1). Events recorded in the PE channel (DL) were restricted to events falling within the FSC gating window using FlowJo 10. $S m=S$. minutum, Smic $=S$. microadriaticum, $S t=S$. trenchii. 
A

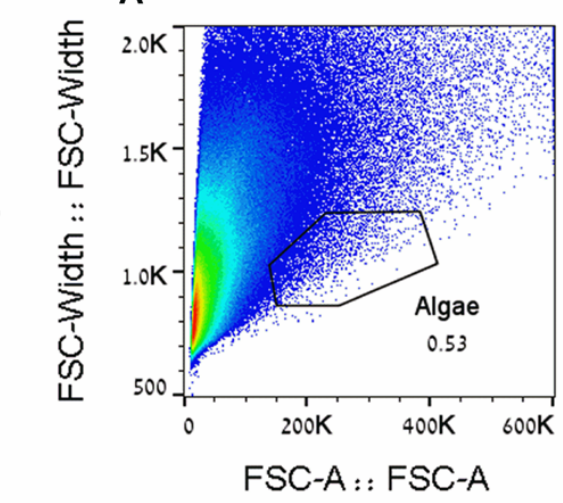

II.
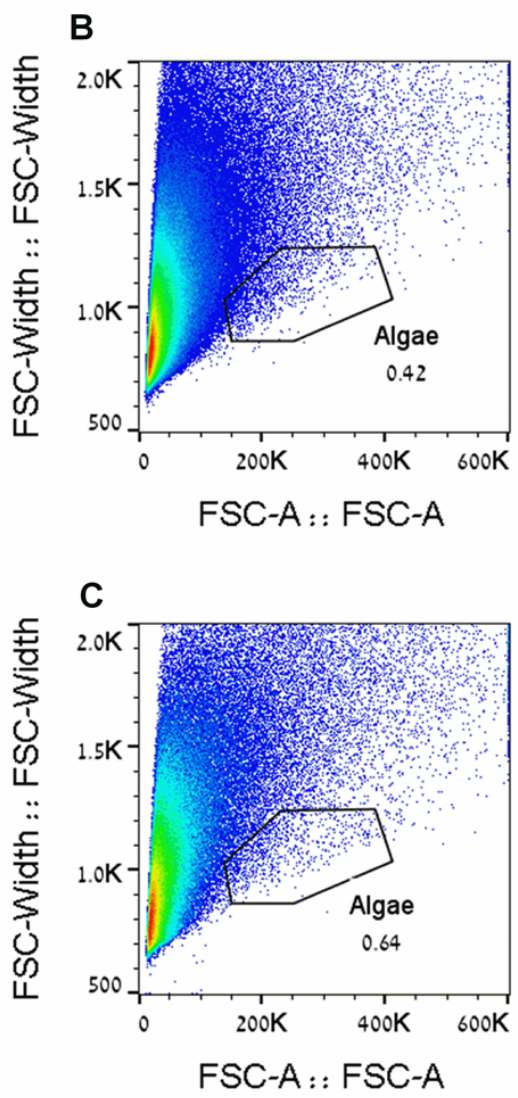

\section{D}

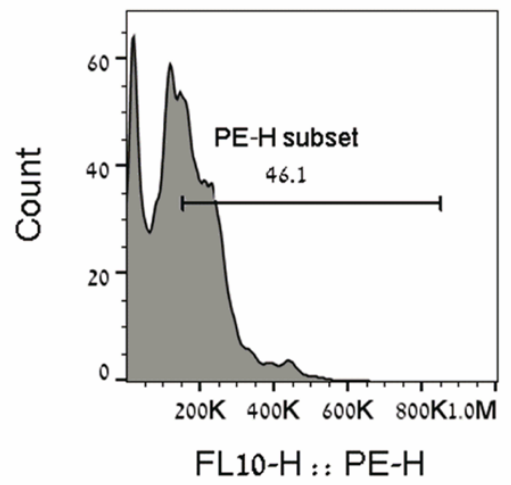

E
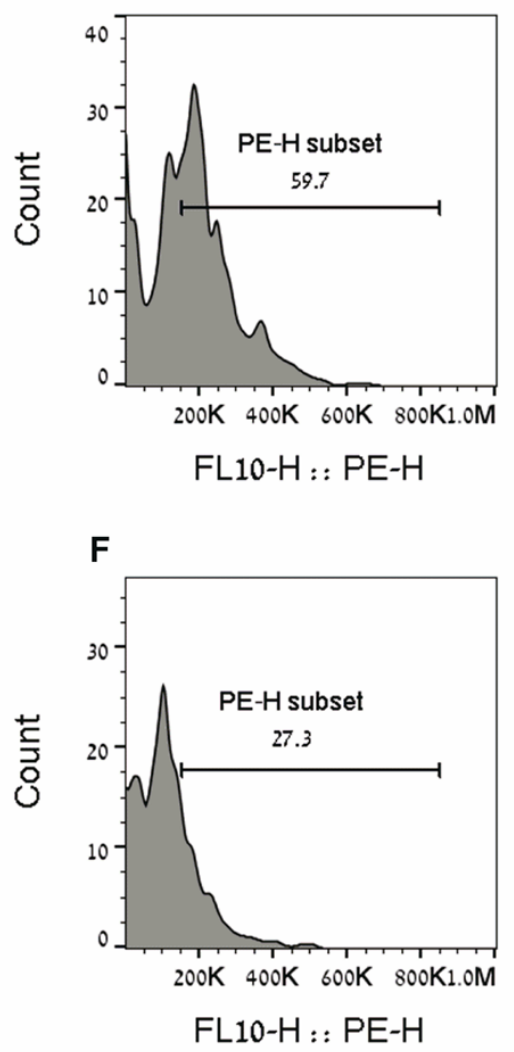

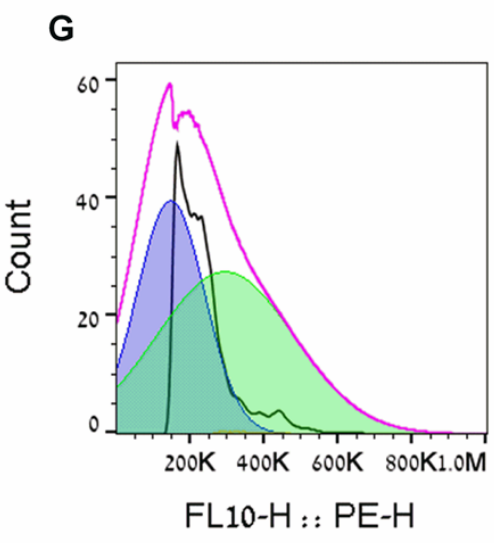

H
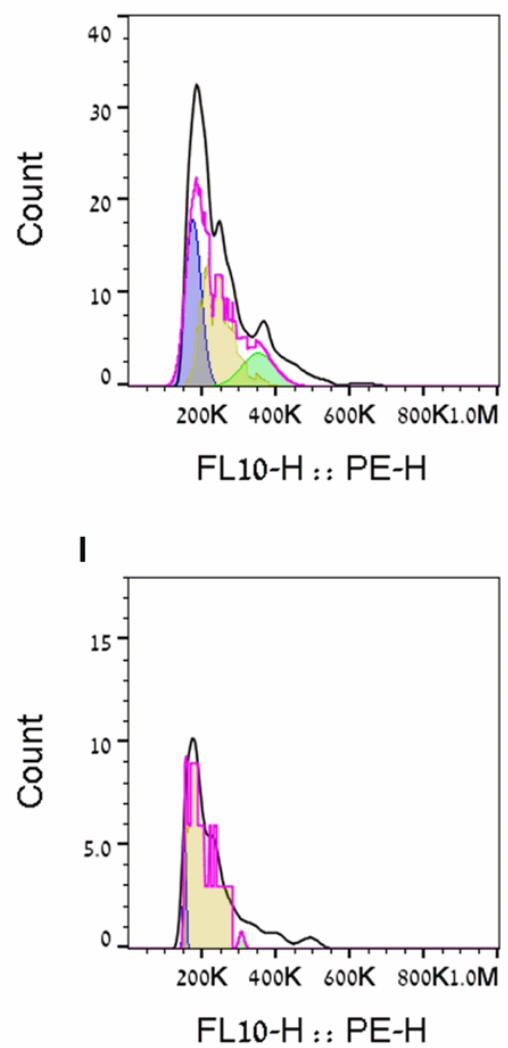

Fig. A6.4. Optimisation of the flow cytometry method. Three control samples were used to quantify algal loss during the washing protocol (See Chapter 5, Materials and Methods), using the combined supernatant of all the samples from each of the three washes used (I-III; $n$ $=28$ supernatants per sample). (A-C) Forward scatter count-area (FSC-A); (D-F) a subset of stained algae using the PE-H channel; (G-I) graphic cell cycle of the algae. The algal signal was gated based on the controls (See Chapter 5, Fig. 5.1). Events recorded in the PE channel (D-I) were restricted to events falling within the FSC gating window using FlowJo 10. 